\title{
Hidden Narratives \\ Augmented Reality to enhance \\ cultural and historical values \\ in the design of Lake Fuxian
}

\author{
By Yanran Li
}

A 120 - point thesis

Submitted to the Victoria University of Wellington in partial fulfilment of the requirements for the degree of Master of Landscape Architecture

Victoria University of Wellington School of Architecture 2021 


\section{Hidden Narratives:}

Augmented Reality to enhance cultural and historical values in the design of Lake Fuxian

Yanran Li

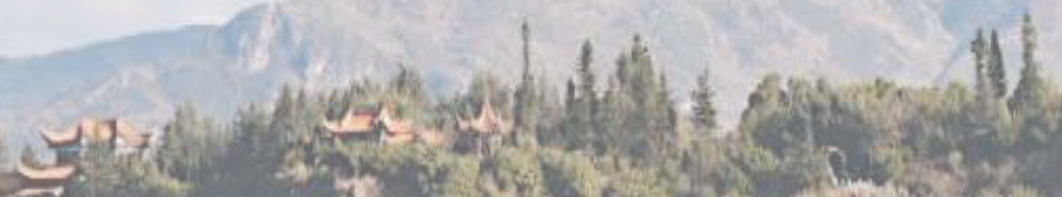
axy is f

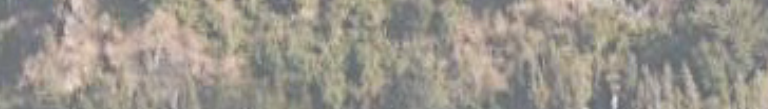
sety

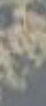

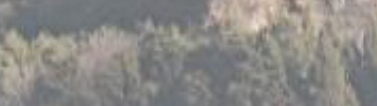


The impacts of globalisation has lead towards the loss of culture, such as overpopulation, forestation and global intergration. The lost culture has mostly been preserved through written text and oral narratives as a way of illustrating a long-gone reality. Similarly, narratives have the power to connect people with imagination and allow them to experience the uniqueness of a specific site in their own terms. However, oral narrative and written text lacked the interaction between communities, or human to Landscape. New technologies have the potential to reconnect these oral narratives or written text with both the wider public and the site.

This research will explore the historical change of Lake Fuxian, from 500 million years ago to present day, through the illustration and experience of Landscape narratives. This research aims to utilize Augmented Reality as a way to physically connect to the past, while still retaining the existing landscape. Augmented Reality has the ability to combine many types of narratives, such as oral, written and drawn, resulting in an educated relationship to Lake Fuxian. Additionally, modernizing these narratives for a larger demographic such as visitors and locals to engage with the landscape, promoting respect for cultural diversity and adaption. 


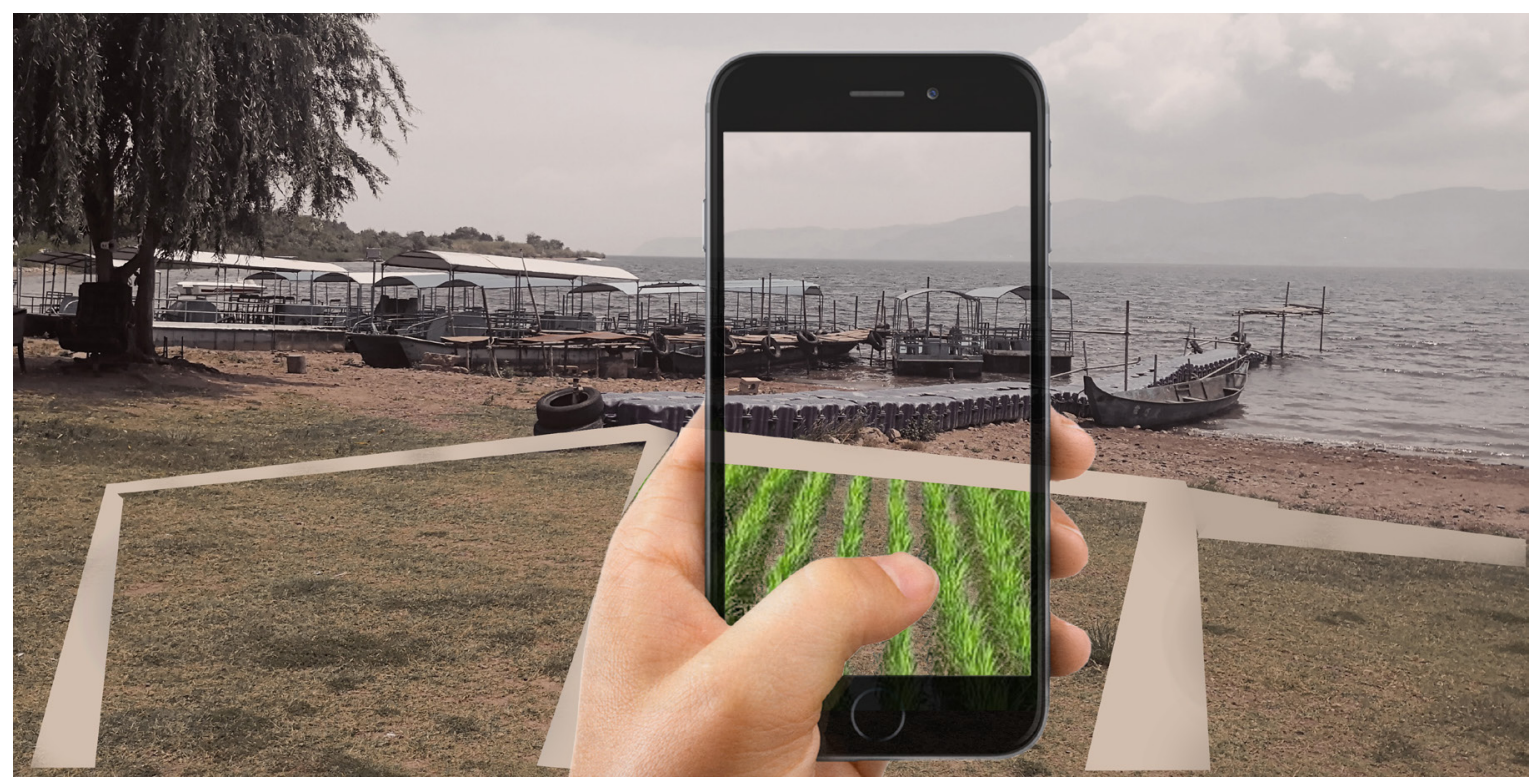

figure $\mathbf{0 1}$. Augmented Reality enhance the design in Lake Fuxian 


\section{$\longrightarrow$ \\ PREFACE}

When I started studying Landscape Architecture, I found myself passionate about how design decisions can inform and enhance human interactions. New technologies such as Virtual Reality and Augmented Reality are becoming popular and, easily accessible in our daily life.

While travelling to traditional Chinese gardens, like those in Suzhou, it is becoming increasingly popular to faciliate visitors with access to Virtual Reality. To facilitate the experience and to expose a hidden reality to how people used and played in gardens 200 hundred years ago. Visitors can understand how people from the past journeyed through the gardens and interacted with ecologies that were abundant during that time. However, the equipment is very expensive, so only a select few groups of people get the opportunity to engage with the past. 
When I had the opportunity to experience the garden through Virtual Reality, I got exposed to the fantastic stories about the site, which in turn allowed me to imagine walking and experiencing that physical landscape. However, as a landscape architect, I want most visitors to share the same experience I had and to get to know the hidden stories as well as to have a chance to interact with the physical landscape.

Furthermore, the extra cost is worth the investment. The understanding of site, and the rich history that came with that, allowed me to envision properties of the landscape through time, which would otherwise have been unknown. As provoking as the VR was, the visualisation was less impactful as it was not shared with everyone that visited the gardens. The knowledge became a hidden secret to only a select few. 
With this in mind, a few questions emerged to help frame this research: How can I use these new technologies to show the hidden stories of the land, which is difficult to understand and visualise by only experiencing the physical landscape? Can I use technology to enhance the interaction of users with the site? Which new technologies are accessible and can be utilised by average working people? After that, I found that Augmented Reality is the most reliable technological option as it is cost-effective, convenient and effective. This has led to the focus of this thesis to be on how augmented reality can enhance the cultural and historical values of site.

The chosen location for this thesis is my hometown, Lake Fuxian. After rigorous research around locations throughout China, Lake Fuxian offered the richest historical roots. The decision was not only because it is my hometown but also the unique stories and the history of the site. Personal connection to the site, via past narratives taught throughout generations, makes this location ideal to represent cultural changes throughout time. This thesis aims to combine personal narratives, the physical site and Augmented Reality together to understand and educate people about cultural change through a design-led research. 


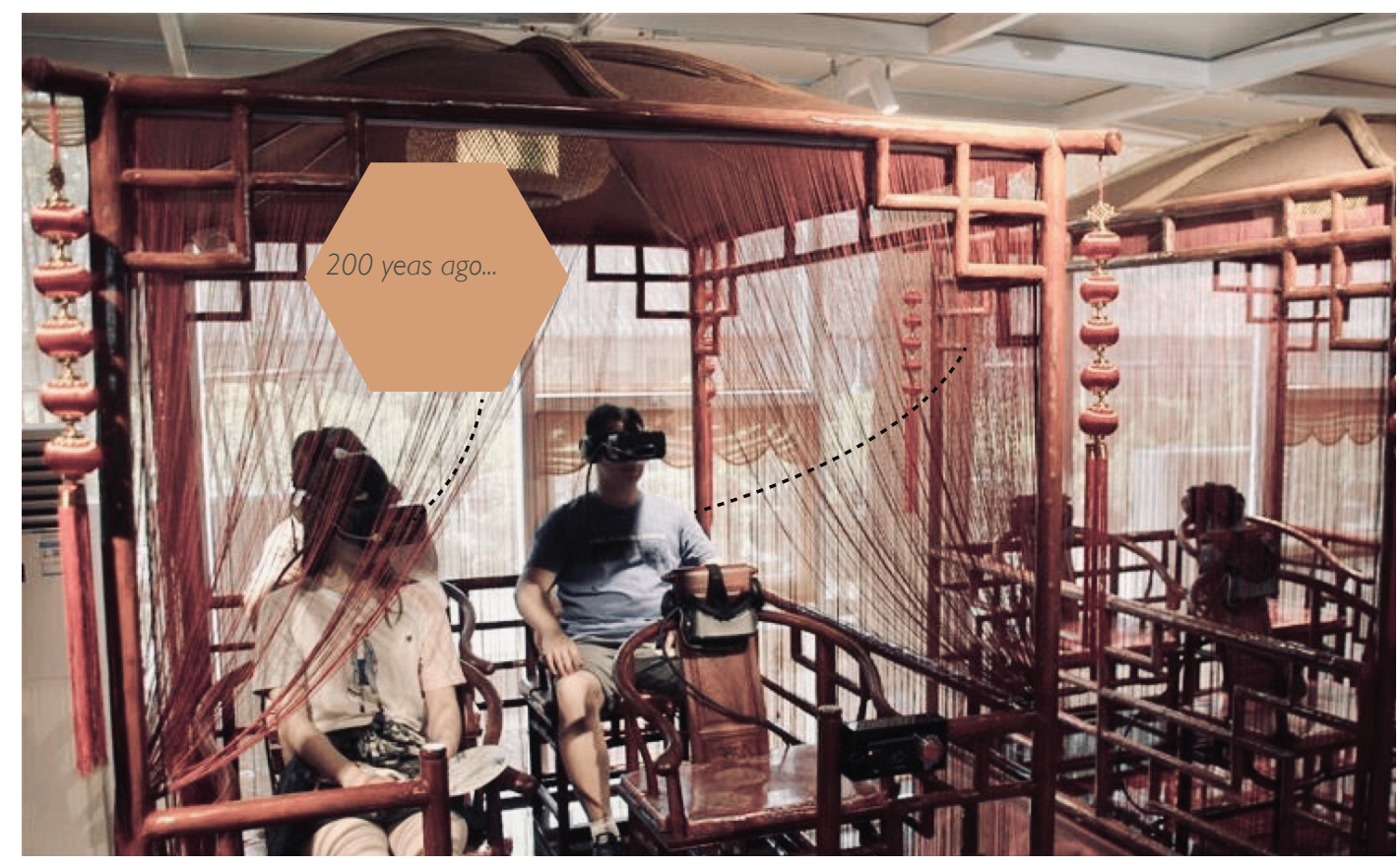

figure 02. Expreicence Virtual Reality in Suzhou traditional Chinese garden 


\section{ACKNOWLEDGEMENTS}

Coming to the end of my university degree and the completion of this thesis, there is a few people I would like to acknowledge.

To my parents in Yunnan Province, China. Thank you for helping me to do the site visit to Lake Fuxian, during this special moment.

To all my classmates, thank you for your continued helps with my study. You made my time at university so memorable.

As for the writing of this thesis. I would like to acknowledge my proof-reader, Michaela. Also, to Natalie, My language teacher in the Student Learning Centre at University, thank you for your language helps for three months.

Finally, to my amazing supervisor Bruno. Thank you for your willingness to help me when I start to study in Landscape Programme, I am really glad that you are my supervisor. I will never forget your help, feedbacks and guidance you have given over four years. 


\section{Contents}

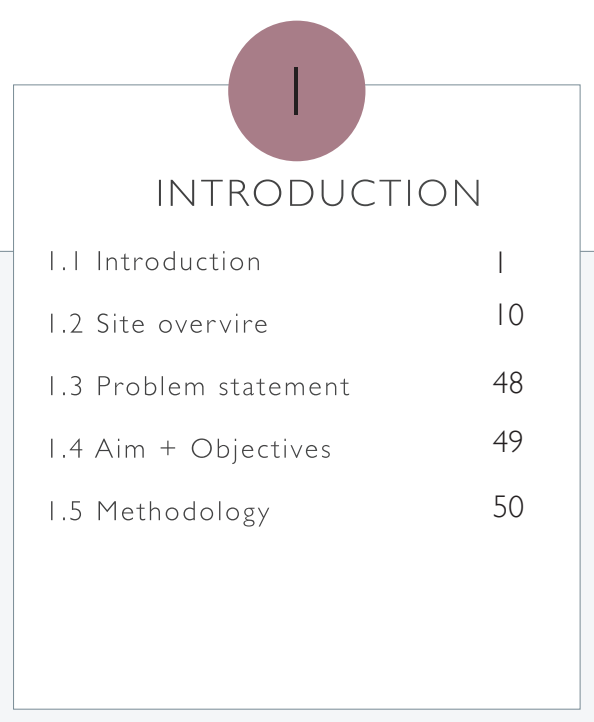

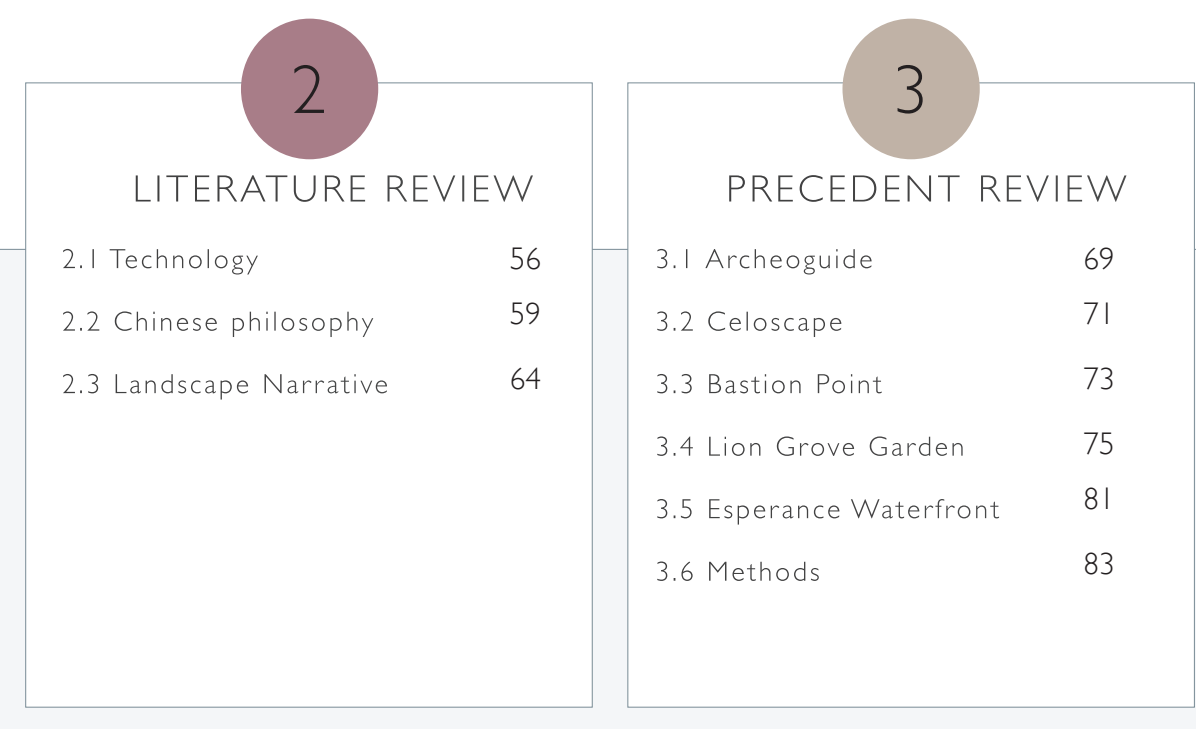

\section{7}

\section{DEVELOPED DESIGN}

7.I Zoom Sites for Site I 164

7.2 Zoom Sites for Site 2

183

\section{8}

\section{CONCLUSION}

8.I Reflection

8.2 Limitations

8.3 Conclusion

196

200

203

\section{9}

BIBLIOGRAPHY

9.1 References

204

9.2 Figure List

206 
4

SITE ANALYSIS

4. I Site context 87

4.2 Site Explorations

a) Site with a vista

b) Site with access to the water

c) Site with cultural significance

4.3 Chosen Sites
5

PRELIMINARY TESTING

5.I site reflection

$|4|$

5.2 Iterations for two sites

143
6

\section{DESIGN}

6.I Design for chosen site । $\quad 150$

6.2 Design for chosen site $2 \quad 156$ 
INTRODUCTION

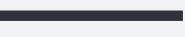




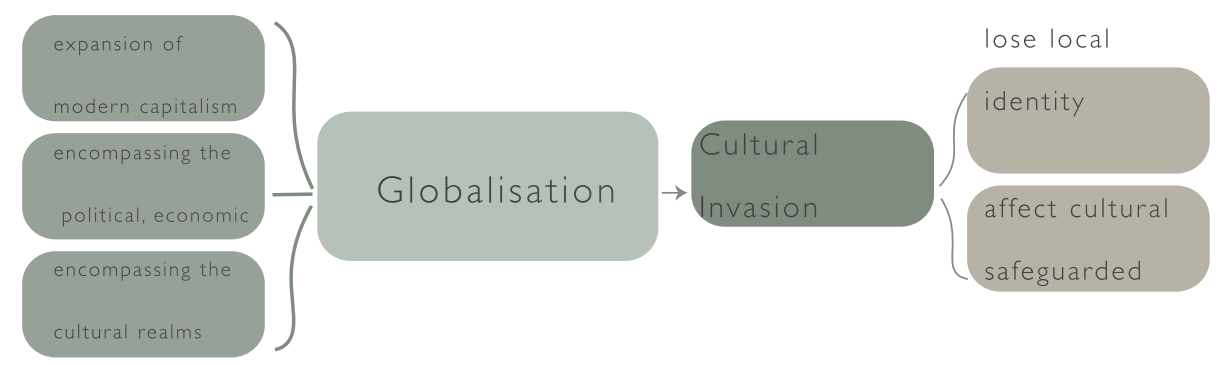

figure 03. globalisation diagram

Globalisation is a global process rooted in the expansion of modern capitalism and encompassing the political, economic and cultural realms. Yet, globalisation equally means the intermingling of different peoples and identities, where powerless minorities are often subsumed under the more powerful minorities(Kaul, 2014). Identity is a social system which works like an organic system and is made up of structure, and cultural values, rules and establish beliefs and practices to which their members are expected to conform (Jones,2005) Cultural identity changes over time and evokes emotions. It is intertwined with power and privilege, affected by close relationships, and negotiated through communication. Cultural identity is derived from formal or informal membership in groups that conclude the local knowledge, beliefs, values, attitudes, traditions, and ways of life(Kaul, 20|4).

Due to the increased impacts of globalisation worldwide, many places have lost their own cultural identity (Kaul, 20I4). Such loss of identity has led to homogenous culture throughout the world, affecting demolished assist local culture and became cultural invasion (Gandhinagar,2014) This has directly impacted how culture is safeguarded. Written records can be considered as a permanent way to preserve the past, compared to oral narratives that fade away quickly (Schudson, 2009). Additional perspectives and individual input allows oral narratives to constantly change and evolve throughout time (Trahar, 2009) This makes oral narratives a more unique and personal connection to the past. 
This investigation leads to the question, "How can the lost history of a site be represented with narrative, through a Landscape Architectural application?"

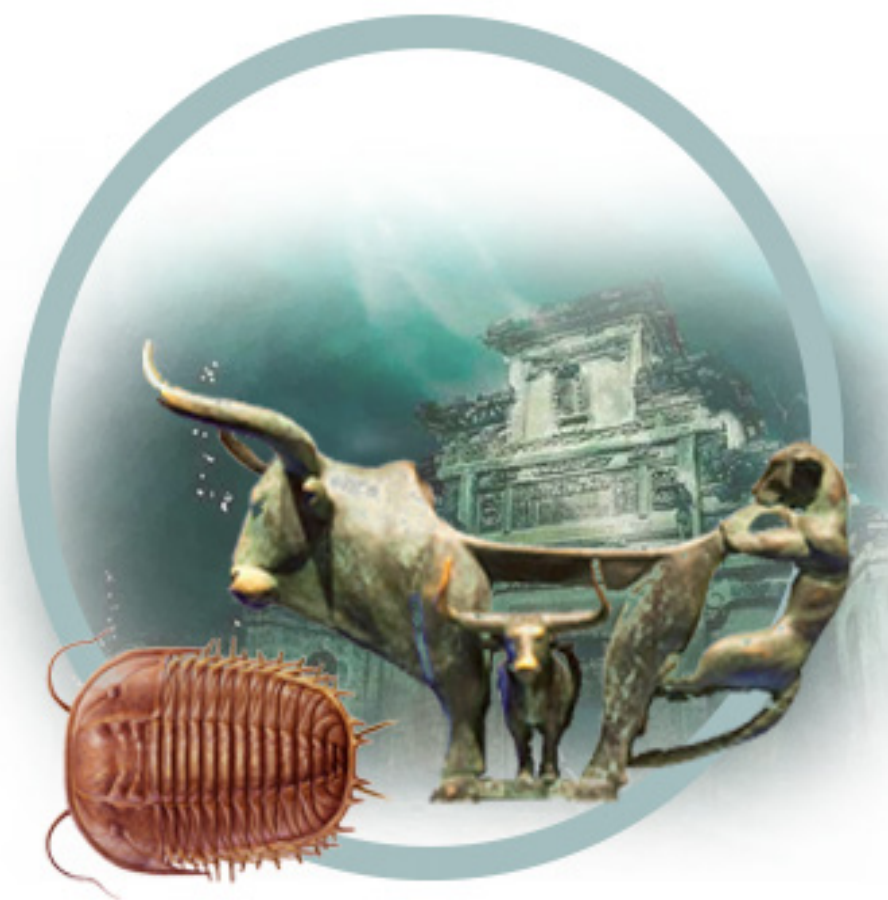

It is well known that the digital world is rapidly expanding. People are relying on information gathering from digital means rather than consulting narratives (Alexander, 2017). Virtual Reality is human-computer interface which can stimulate realistic environments, and people have used Virtual Reality equipment to see the virtual world(Zheng et al, 1998). Unlike Virtual Reality, Augmented Reality blended "real world elements" and "virtual elements" might also have hearing, touch and smell (Borgeat, 2016) Currently, Augmented Reality is the emerging technique that can reconstruct history with virtual visual stimuli (Noh, 2009).

figure 04. Unique history and culture in Lake Fuxian 
Adding the narrative layers into the conceptual space of the digital world allows a wide range of people to understand lost history.

This research will focus on Lake Fuxian, located in the southwest of China. It holds a rich historical presence, through initial settlements belonging to the Ancient Kingdom of Dian at the edges of the Lake, around 2000 years ago. Evidence of the Kingdom can only be understood through an investigation of the architectural remnants at the bottom of the Lake.
Digging deeper, fossils around the site paint a clear picture of the fauna and flora of the area, making it one of the most well preserved areas from the Cambrian period(54I million years ago -48I million years ago)(Huang, 2012). 


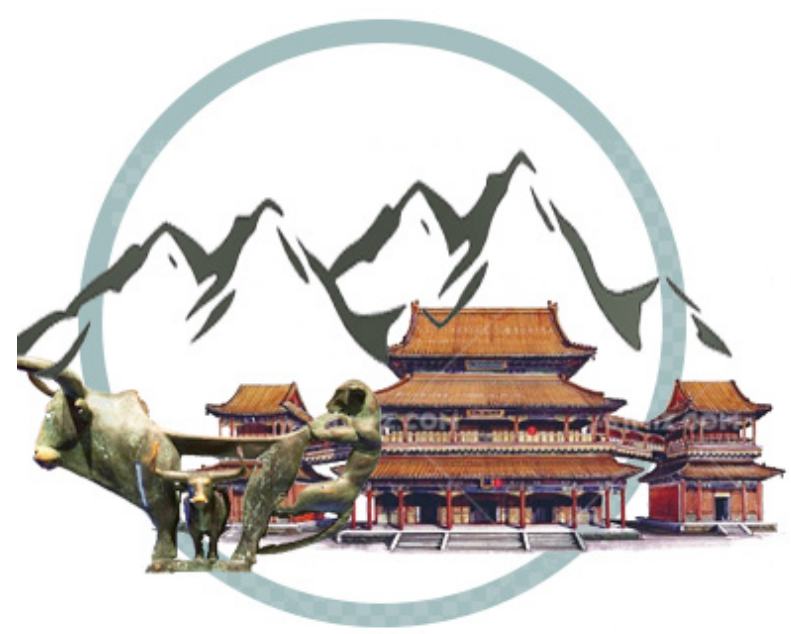

figure 05. Dian's culture

The shores of this Lake do not reflect this history, nor the passage of time that it has developed from. It has become exclusive information, known only to individuals who decide to seek themselves through written narratives. After visiting the site, people do not notice the cultural differences between these lakes with other lakes in China, and this Lake became one of the sites that has lost its own identity.

Despite being lost, Dian culture holds a lot of importance to cultural practices today. It is vital to re-introduce these lost traditions and narratives by establishing values back into the landscape. This thesis aims to utilise Augmented Reality as a tool to allow people to better understand the cultural landscape of the past, with visual connections to the present. Lake Fuxian will be brought to life by enabling narratives over time to be interactive and informative to a wide audience. 


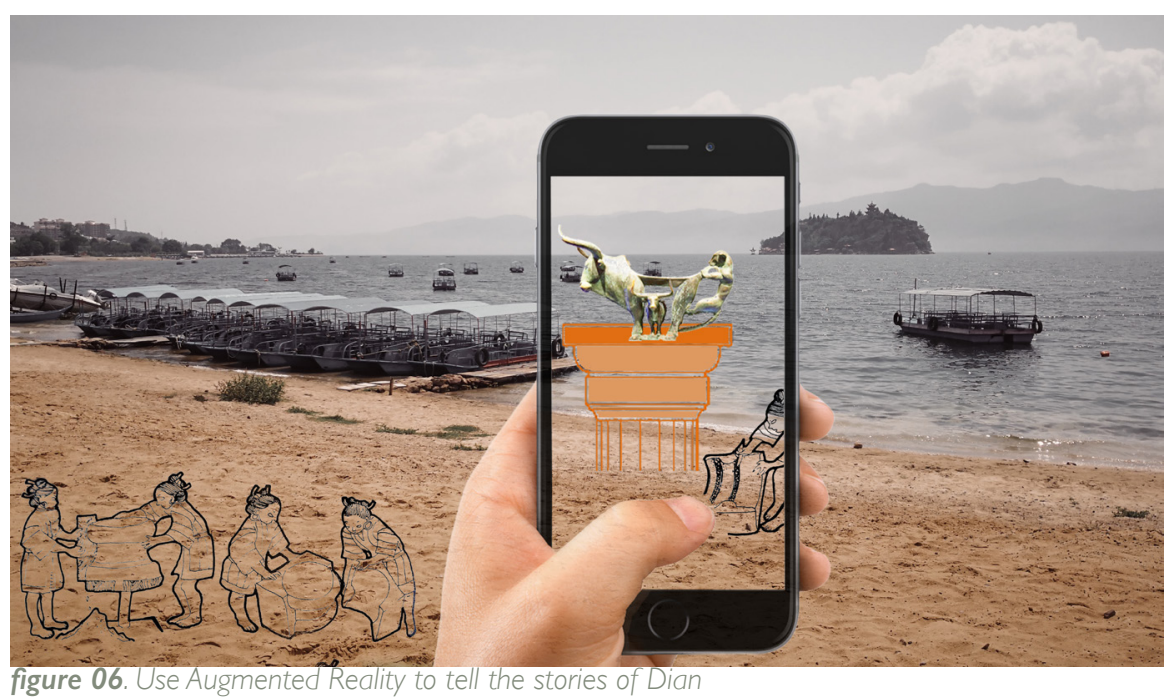

This design-led investigation adopts an iterative method, which brings together the relevant findings of the literature with the best practice knowledge acquired through precedent analysis. Both literature and precedents were selected based on connections to Augmented Reality, Narrative Landscape and Chinese philosophy. This is to gain an understanding on how to create hybrid spaces. From there, the site was analysed through different angles, which included looking at the biophysical and tangible layers of that landscape as well as the intangible and cultural values. Apart from different mapping aspects, an extensive visual and photographic analysis of the lake and its shores will be conducted. 
Additionally, digital modelling was executed to allow numerous concepts to be explored spatially. The use of Augmented Reality allowed the refinement of design proposals by grounding them into the landscape. By introducing a design that will enable people to use their phone to explore the hidden layers of information, a more enhanced and engaging experience can be delivered.

Overall, after the design research, the identity of Lake Fuxian will be highlighted and that attract more visitors to come and use the site. Visitors would have an educational and exciting journey through the narrative landscape around the lakefront. Then, after the journey around Lake Fuxian, visitors or locals will obtain the knowledge about the paleontology, geography, biophysical, history of the Dian culture of Dian of Lake Fuxian by following a timeline.

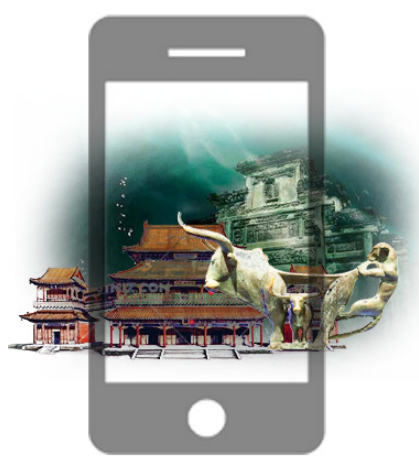

figure 07. Research diagrams 


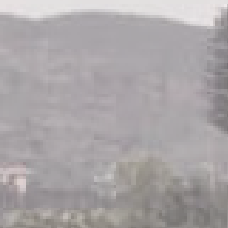

t.

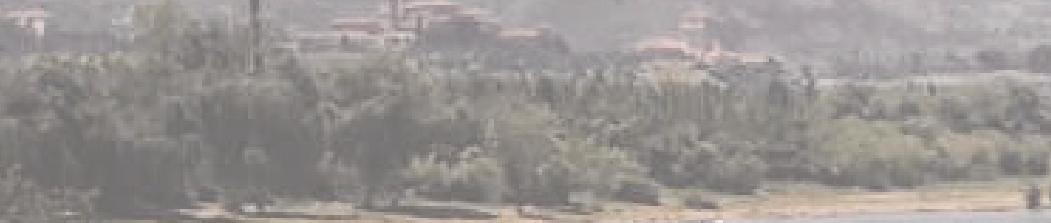

\section{n

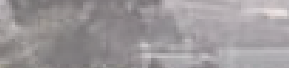

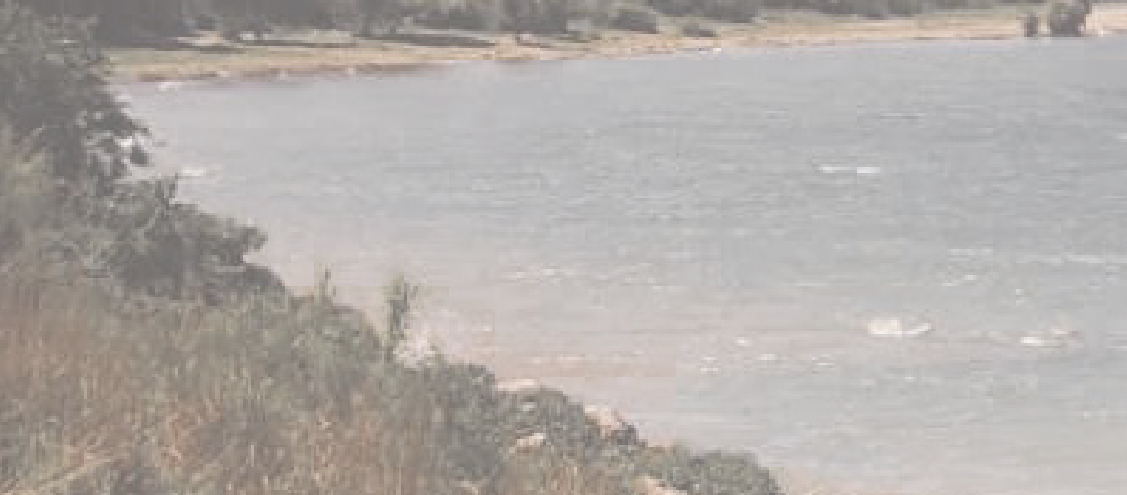

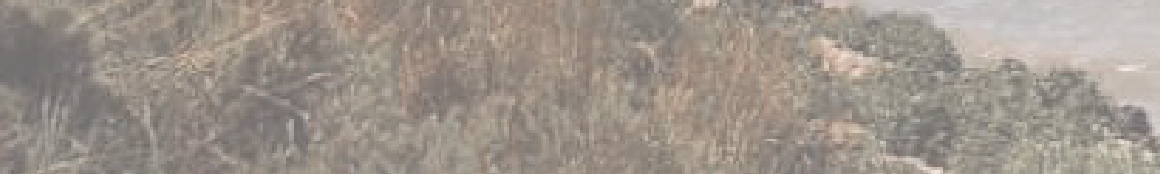

20.

S.

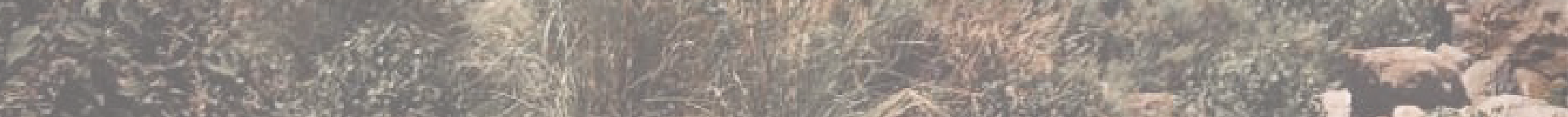
F.

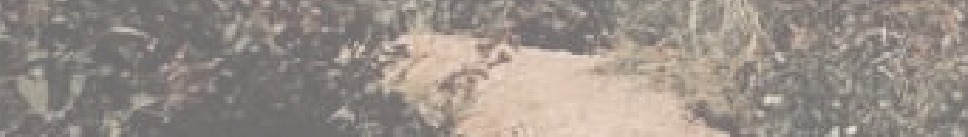

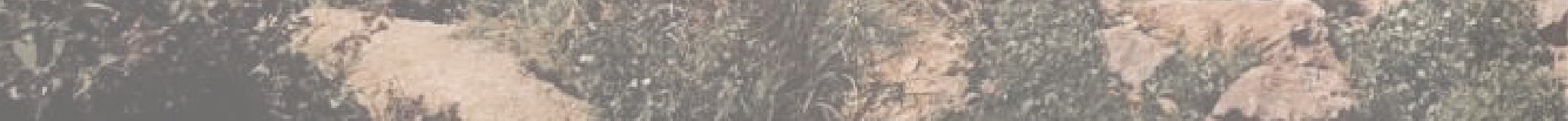

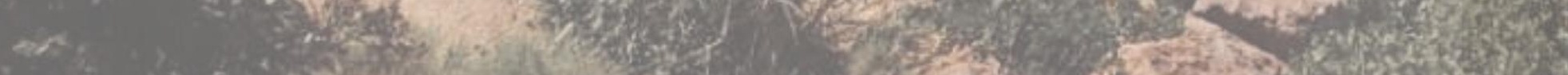
W.

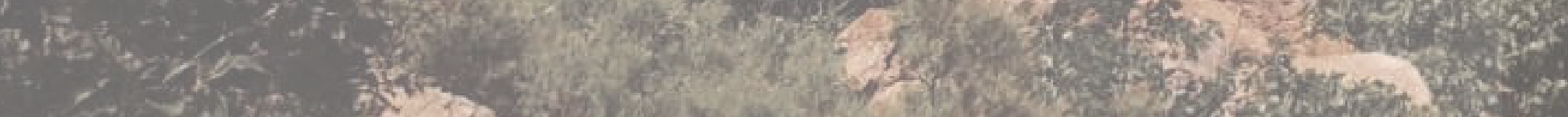

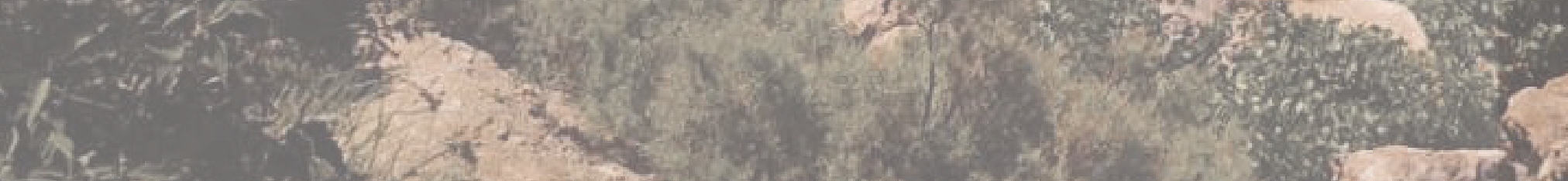

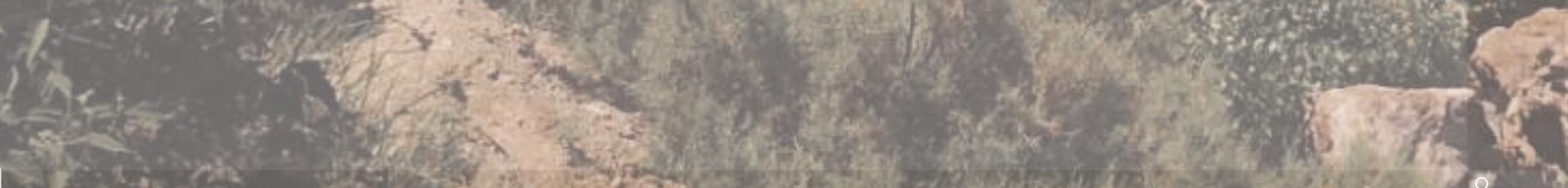

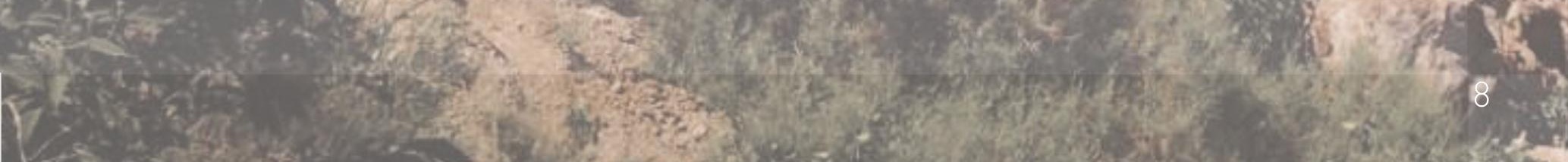

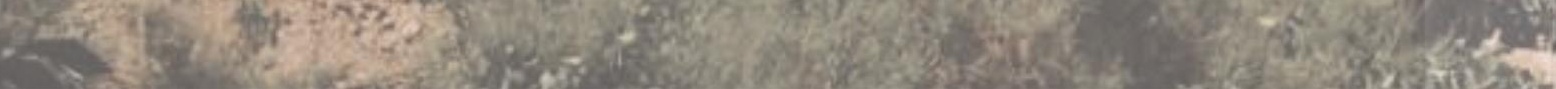

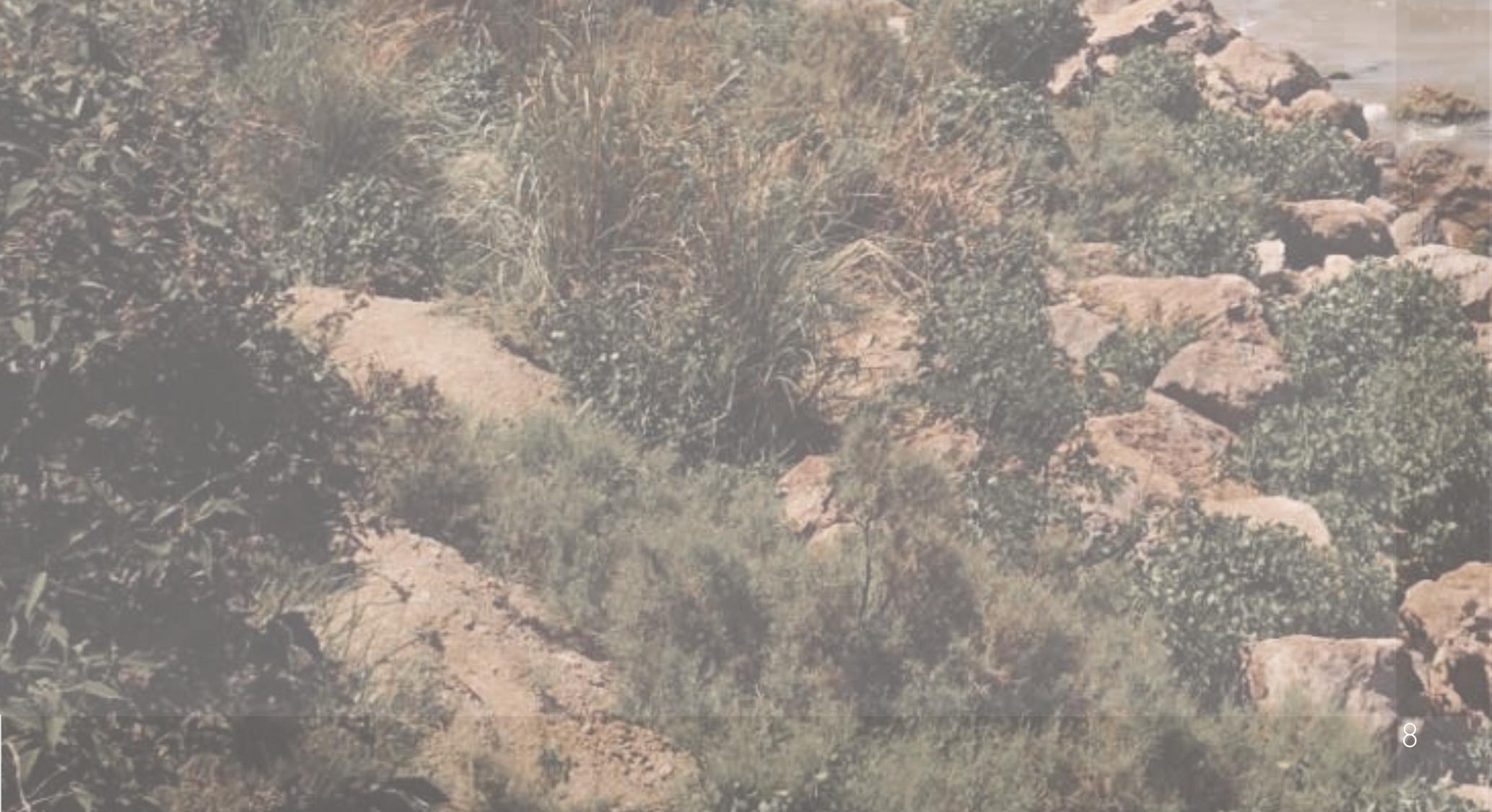




\section{SITE \\ OVERVIEW}




\section{contents}

LISTORY




\section{TIMELINE}

Establish diplomatic relation with China 2228 years ago

Cambrian Period

500 million years ago

233 I years ago

Dian Settlement in Yunnan

2128 years ago

Dian conquered by China

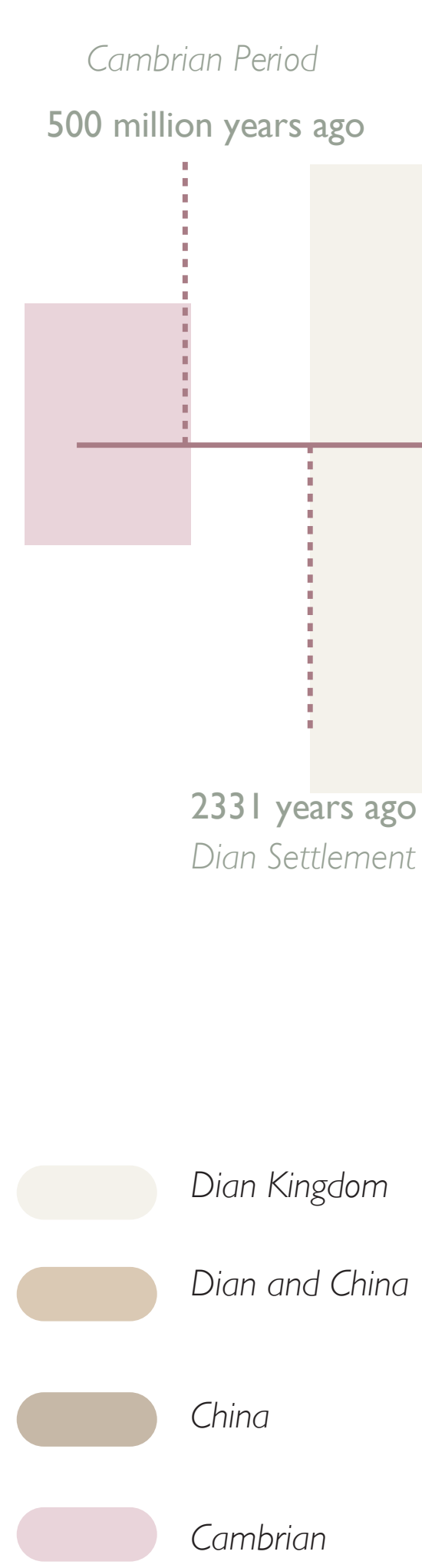

Final king of Dian died 2106 years ago

figure 09. Timeline of Dian 
Urban start around the lake 200 years ago

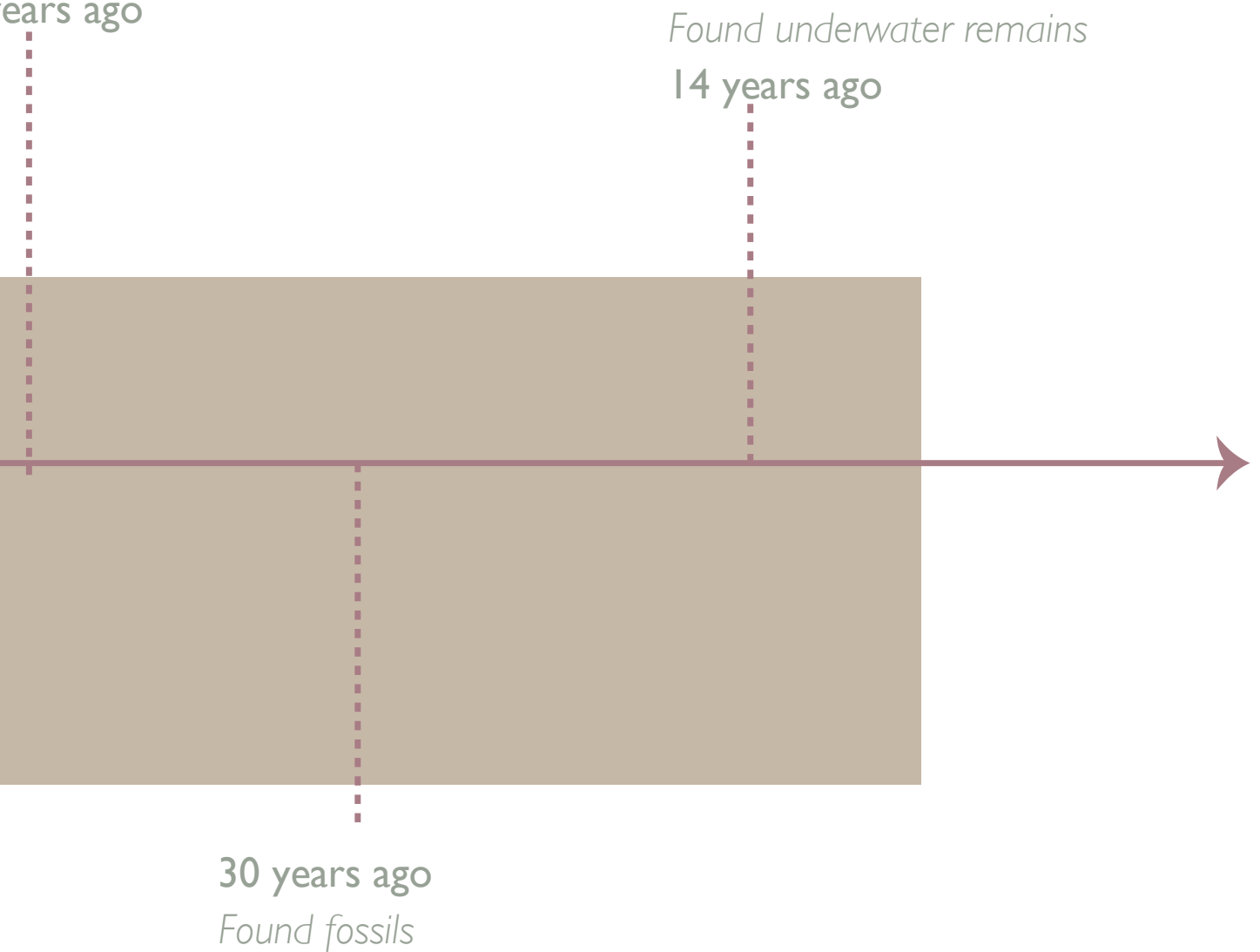

2000 years ago

cture slide to lake 


\section{HISTORY}

Dian is an ancient kingdom established by the ethnic minorities in Southwest China, mainly distributed along the lakeshore of central Yunnan. (Zhang, 2005)A large number of archaeological data confirm that the Dian kingdom appeared no later than the middle and late Warring States period, 2200-2300 years ago. (Zhang, 2005) Then, during the West Han Period, 1800-2000 years ago, King of the Han (China) conquered Dian and joined it to the rest of China (Zhang, 2005).

The kingdom of Dian and its culture have disappeared in Yunnan's history, there are limited records of the ancient Dian state in old literature records of China. Ancient Chinese texts refer to the Dian kingdom as one of the most powerful entities in south-western China (Yao, 2005). Archeologists have found dozens of ancient tombs around inaccessible sites of Lake Dianchi and Lake Fuxian. This has generated a wide interest in studying the culture and history of the Dian Kingdom. (Zhang, 2005) 


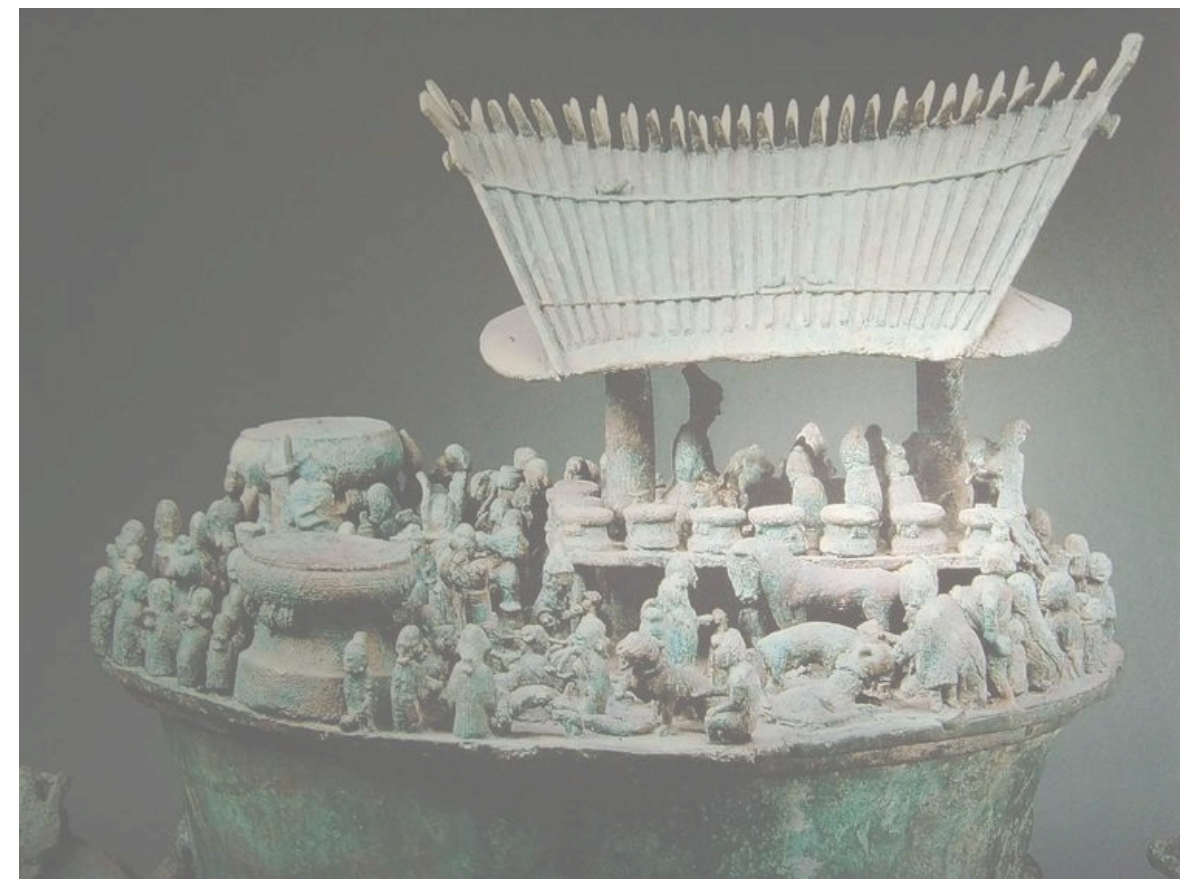

figure 10. Bronzes illustrates the architecture and people with Dian 


\section{NAME}

The name of Lake Fuxian, Fuxian 抚(Fu) 仙(Xian) means a site that is close paradise. As the words explain 抚(Fu) means touching, and 仙(Xian) means deity.
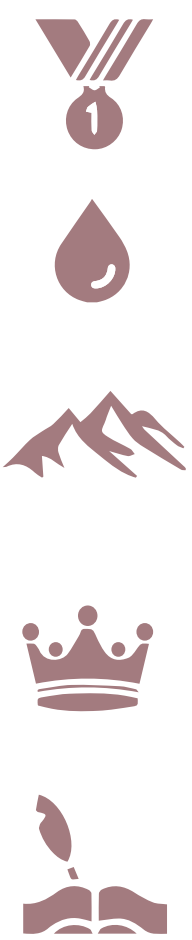
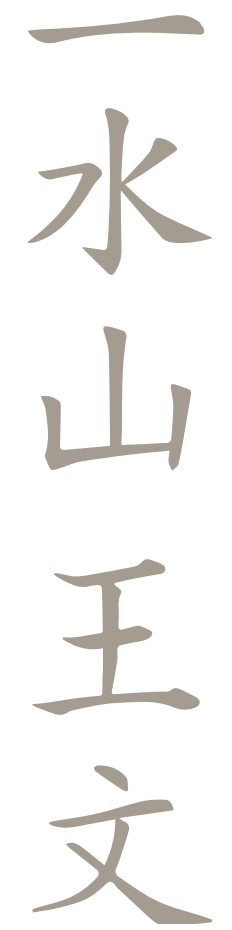

From 700 years ago, the primary county managing Lake Fuxian was named “溦江”(Cheng Jiang). Cheng in Chinese means sink, and as the structure of “溦” it is combined with five independent Chinese words. 


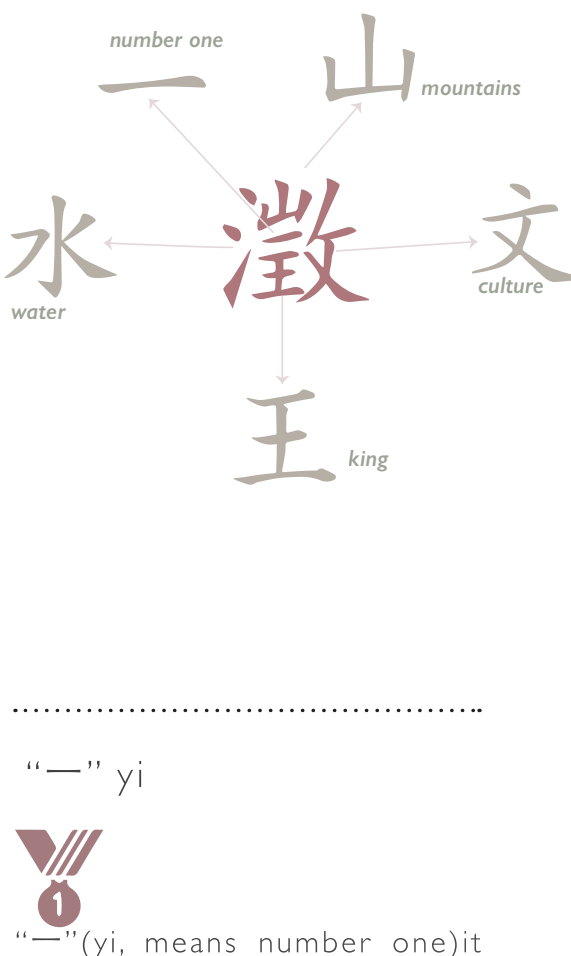

means the lake is special in the world, and it is different from other lakes.

“水” shui

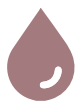

figure II The meaning of the name of Chengjiang County

“水”(shui, means water) it means this place has plentitude of water.

"山”shan

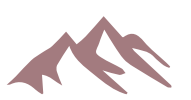

“王” (wang, means king) it

“山”(shan, means mountain) means the king is living in this space.

means the location of the place has few mountains. 


\section{GEOLOGY \& BIOLOGY}

As stated by many scientists, the world is about 4500 million years old(USGS, 1997). Through that time, it has changed enormously because of evolution of biology (Hou et al,2012) "It has been divided up into more manageable packets of time, comprising four eons, the Hadean, the Archean, the Proterozoic, and the Phanerozoic. The Hadean, Archean, and Proterozoic are jointly termed the Pre-cambrian" (Hou et al,20l2, pl5) The fossils found in Chengjiang mainly record the paleontology during the Cambrian era. Then, the site has inscribed to the UNESCO World Heritage in 2012.

According to Vannier \& Martin (2017), the Chengjiang ecosystem has highly distinctive red and orange hues by comparing it with in contrast to other biotas(Figure 12). It might be because it of the connection to mudstone, which is unique from Sedimentary rock, so therefore the biota's colour looks special (Hou et al,20|2).
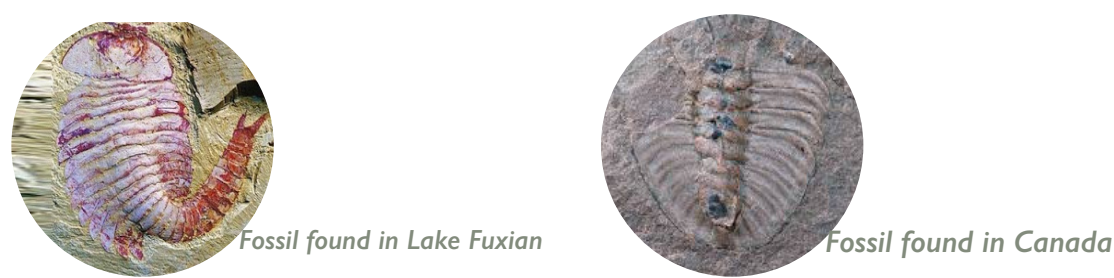

figure 12. Comparison of Biota in Lake Fuxian and the Fossils in Canada. 


\section{Yuxi City}

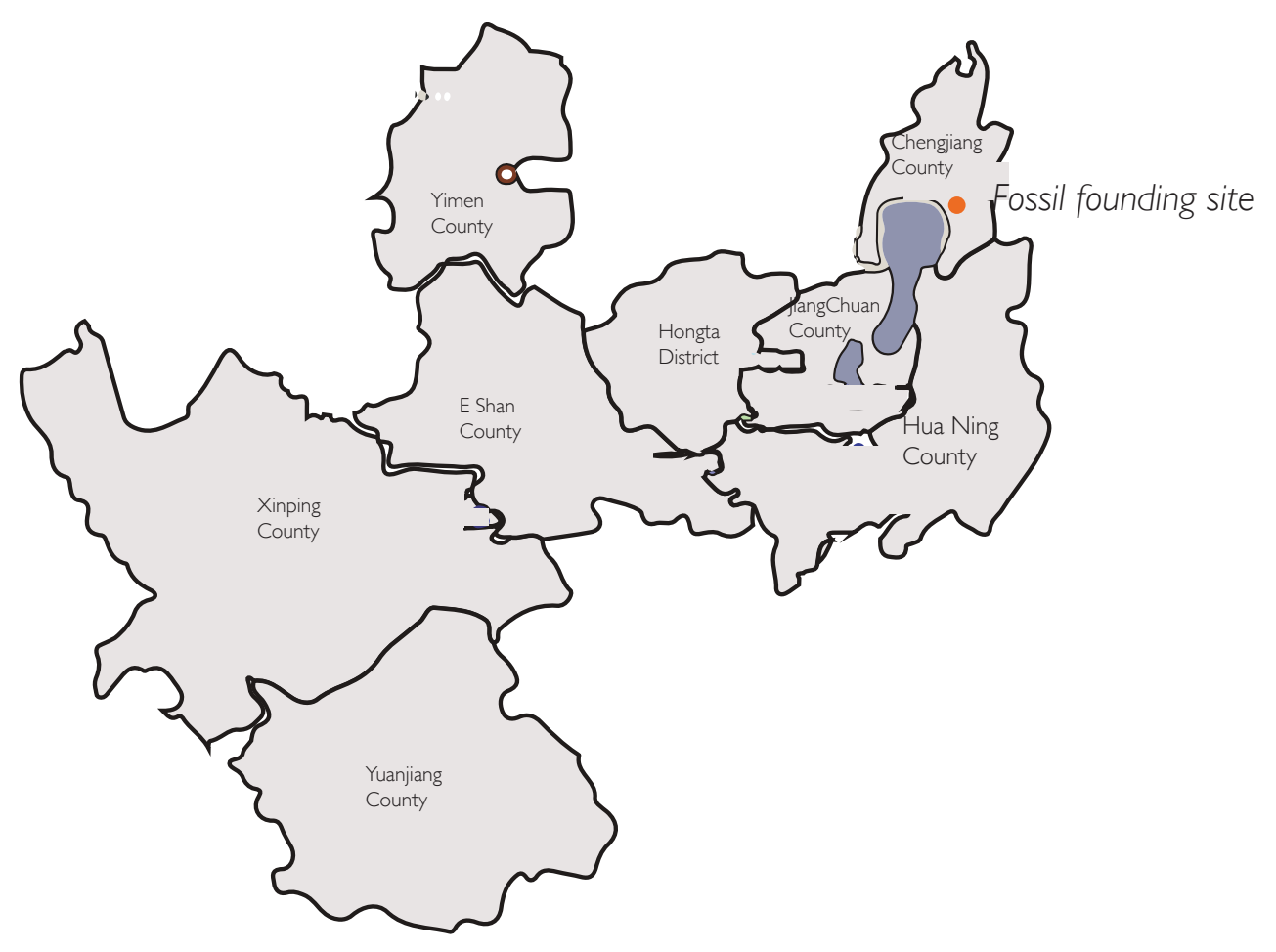

figure 13. Map of Yuxi city, Fossil Site 


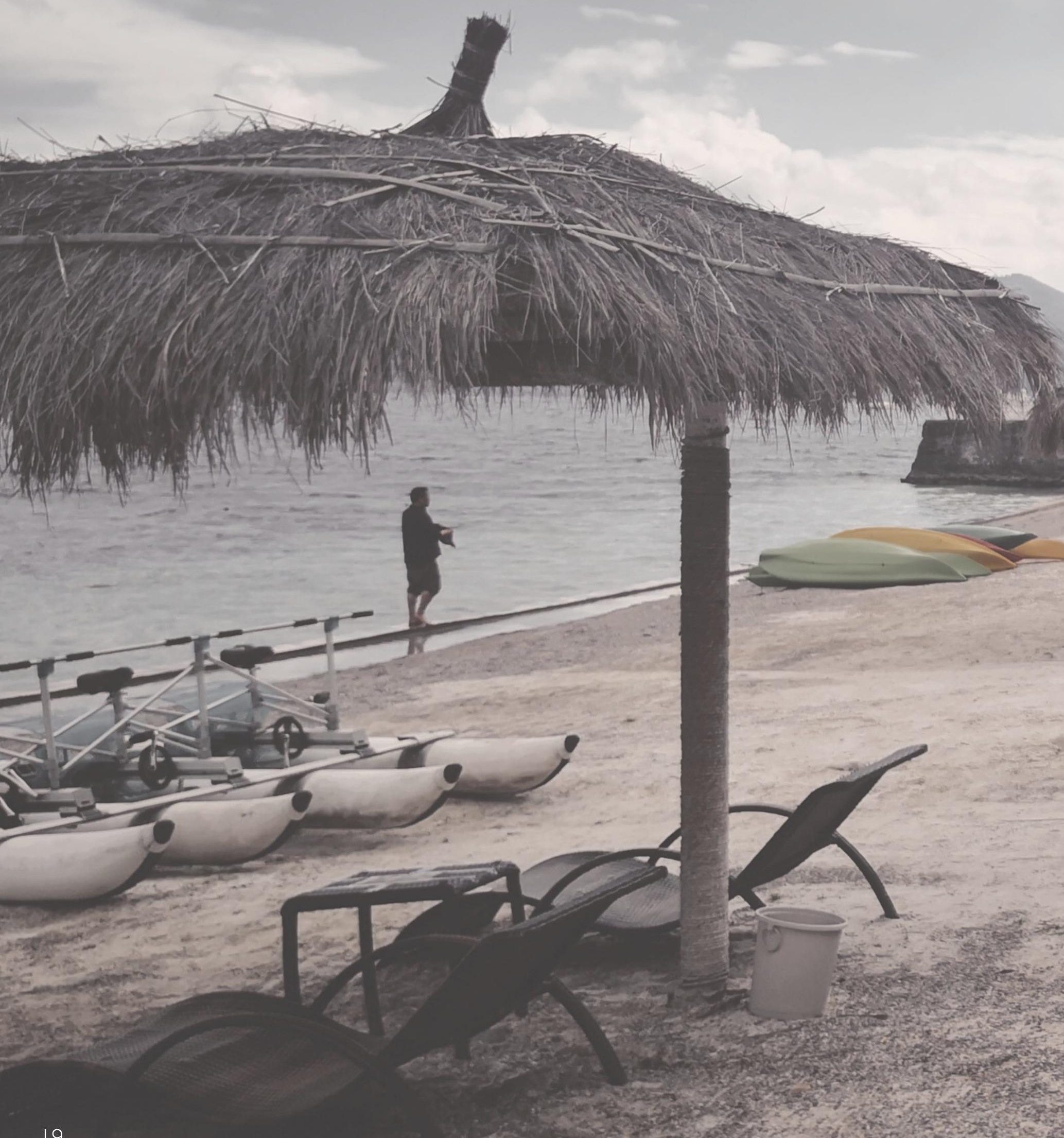




\section{CLIMATE \\ CONDITION}

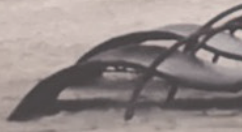




\section{LOCATION}

Lake Fuxian is located in the beautiful Province of Yunnan in China(Figure 15). Yunnan Province is positioned on the border of China, which is close to Myanmar, Laos and Vietnam. Yunnan has the largest number of ethnic minorities in China; every minority has its own culture (Hillman, 2003) The culture in Yunnan is diverse including a myriad of both international and domestic cultures.

Lake Fuxian stretches out through Chengjiang, Jiangchuan, and Huaning Counties in Yuxi City, Yunnan Province. It claims 212 square kilometres and ranks as the third-largest lake in the Yunnan Province, the second deepest lake(155m) in China(People's Government of Yunnan Province, 2016). The quality of water in Lake Fuxian is identified as first level, which is one of the best water quality levels in China. 


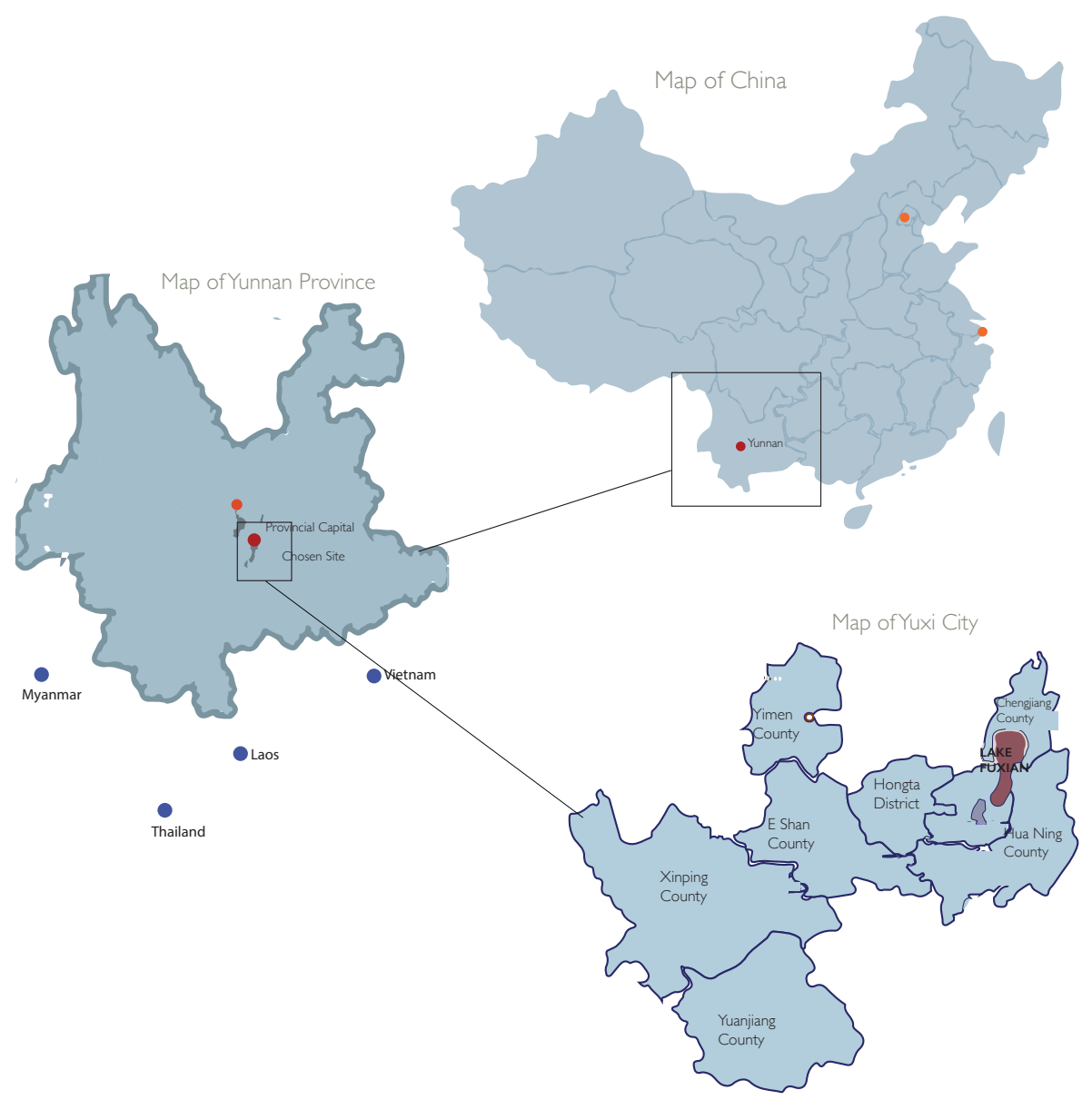

figure 13. Location of Lake Fuxian 


\section{ENVIRONMENTAL CONDITION}

Due to the influence of tectonic basin, the terrain of Fuxian Lake with high altitude on its surrounding $(2439 \mathrm{~m})$, and low in the lakefront(I722m), with large relative elevation difference (Chen, 2015). The lake is surrounded by mountains in the East, South and West, and connected with Chenjiang plain in the north. The soil on flat land around lakefront and the flat plain on North of the Lake is fertilized by floods (Chen, 20I5). (See figure 14) It is easy to irrigate the land by catchment water. The recharge of the lake mainly relies on local rainfall and catchment surface runoff (figure 15). In addition, the landcover surround the lake are tussocks, boscage, and coniferous forest(Dai et al, 2016). According to Dai et al(2016, P4l9) "The land-use type in the catchment is all along forests and water, which has changed to a certain degree in recent years due to the phenomena of reclamation of land from lakes and forests, overdevelopment of tourism resources, and diversed functional land for construction" Since the ability of lake water to repair itself is relatively weak, people need to pay attention to prevent water pollution. 

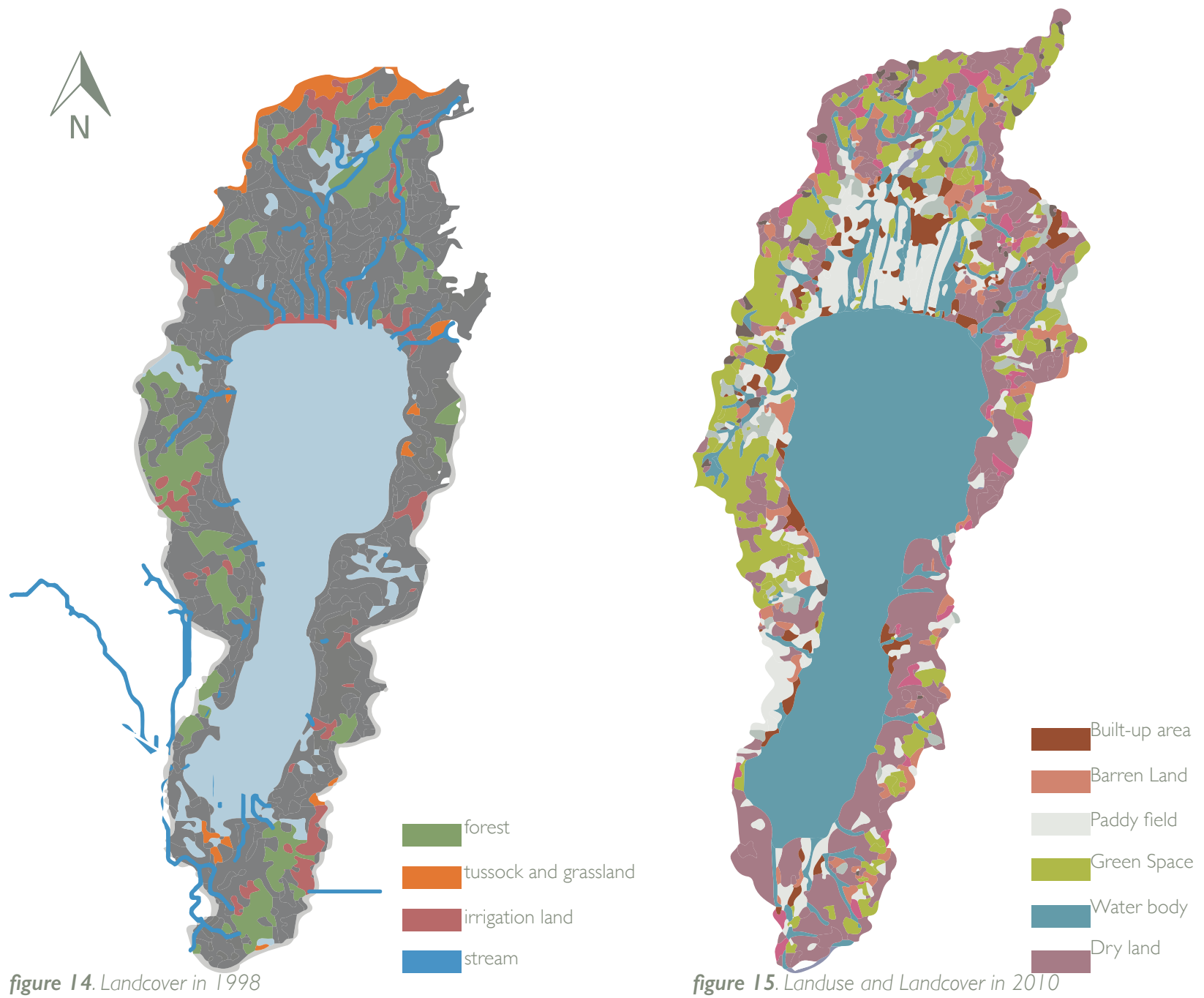


\section{WEATHER}

The weather in Lake Fuxian can be described as average. Lake Fuxian has a humid subtropical climate where in the summer it is humid and in winter it is dry (Fuxian Climate, 2020)(figure 16). Lake Fuxian is a plateau lake, it located $1790 \mathrm{~m}$ above sea level (Huang, 20I5). The average annual temperature in Lake Fuxian is $17.1{ }^{\circ} \mathrm{C}$ (Fuxian Climate, 2020). Frost may occur in winter but the days are generally warm, up to around 17 ${ }^{\circ} \mathrm{C}$ (Fuxian Climate, 2020). During summer, there is minimal rainfall with the average temperature set at $26{ }^{\circ} \mathrm{C}$ (Fuxian Climate, 2020). To conclude, the weather in Lake Fuxian is not cold during winter and not hot during summer, it is like spring all year around(figure 17). 


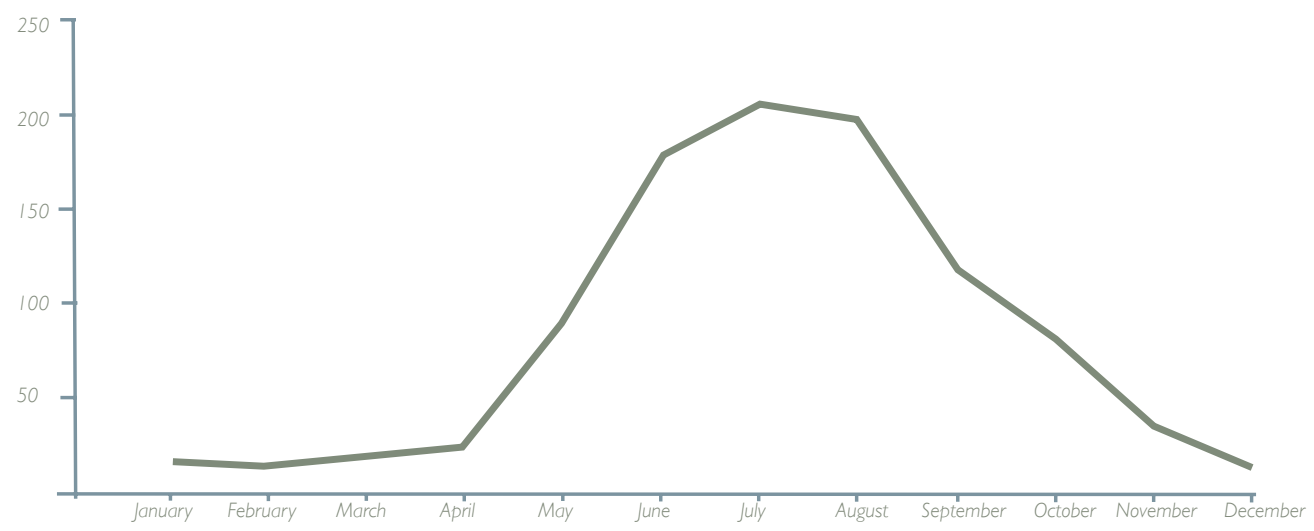

figure 16. Average rainfall in Lake Fuxian

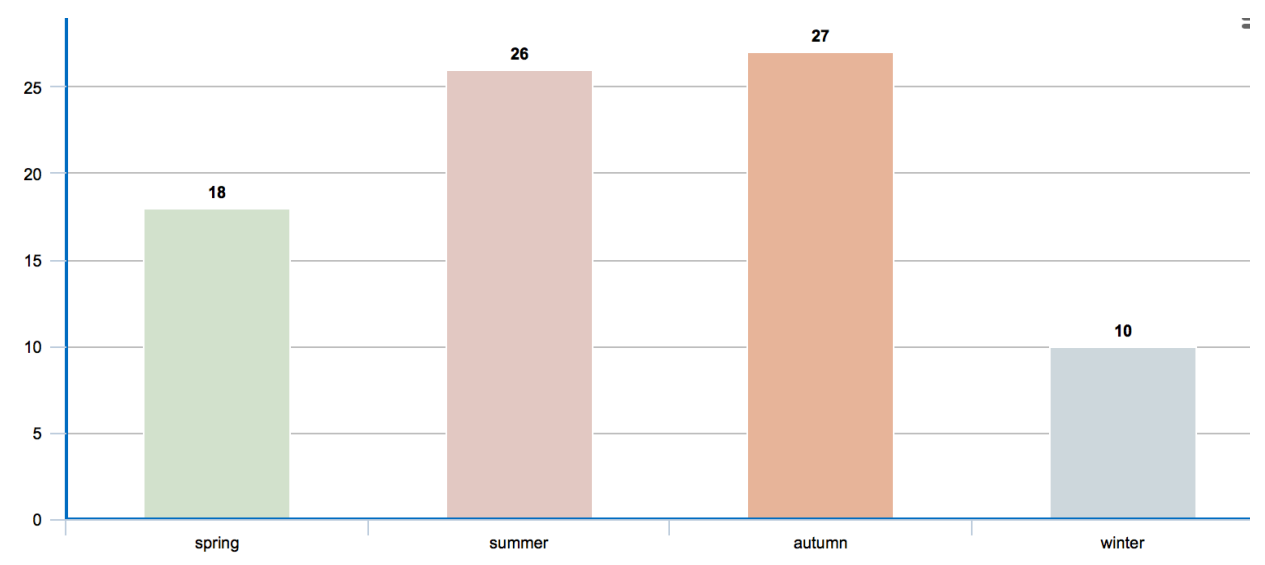

figure 17. Average temperature in Lake Fuxian 


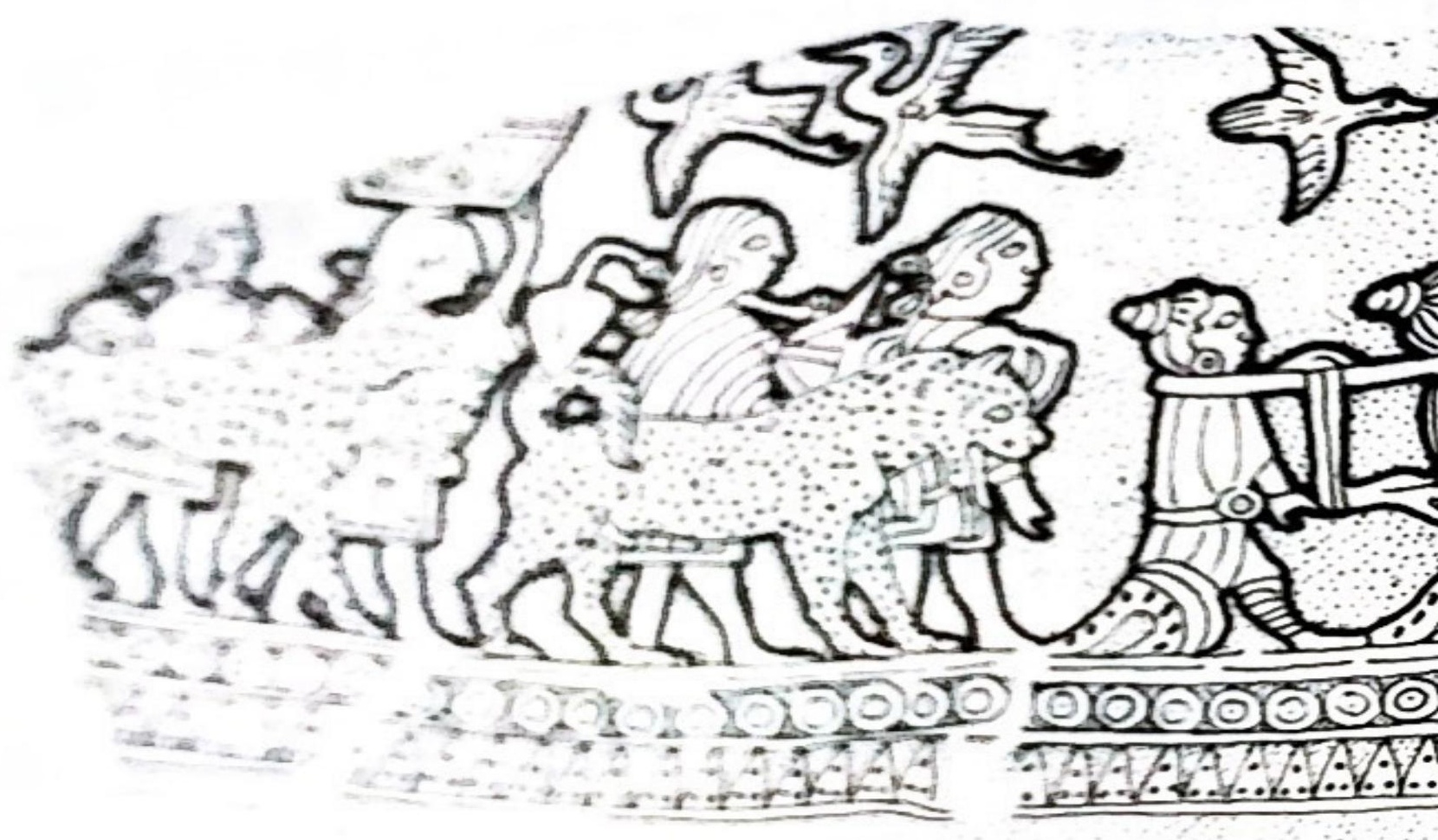

figure 18. The king of Dian with his tiger ,Dian people is holding rice

\section{AGRICULTURE}

There are few plain land around the lake, Dian people decided to settle around Lake Fuxian because the environment and warm weather is positive for developing agriculture. Dian people during that period started to use sediments eroded from the catchment to fertilised the land(Zhang, 2005). Also, they used relevant developed knowledge to make water run off the land by reorganising the catchment of the Lake(Zhang, 2005). Dian people mainly plant staple food crops of rice around the Lake, as the warm weather and enough water run-off can support rice growing well (Zhang, 2005). 
There were four main uses of cattle for the Dian Kingdom:

- The first is for sacrifice.

- The second is for consumption, mainly meat and dairy products(figure 19).

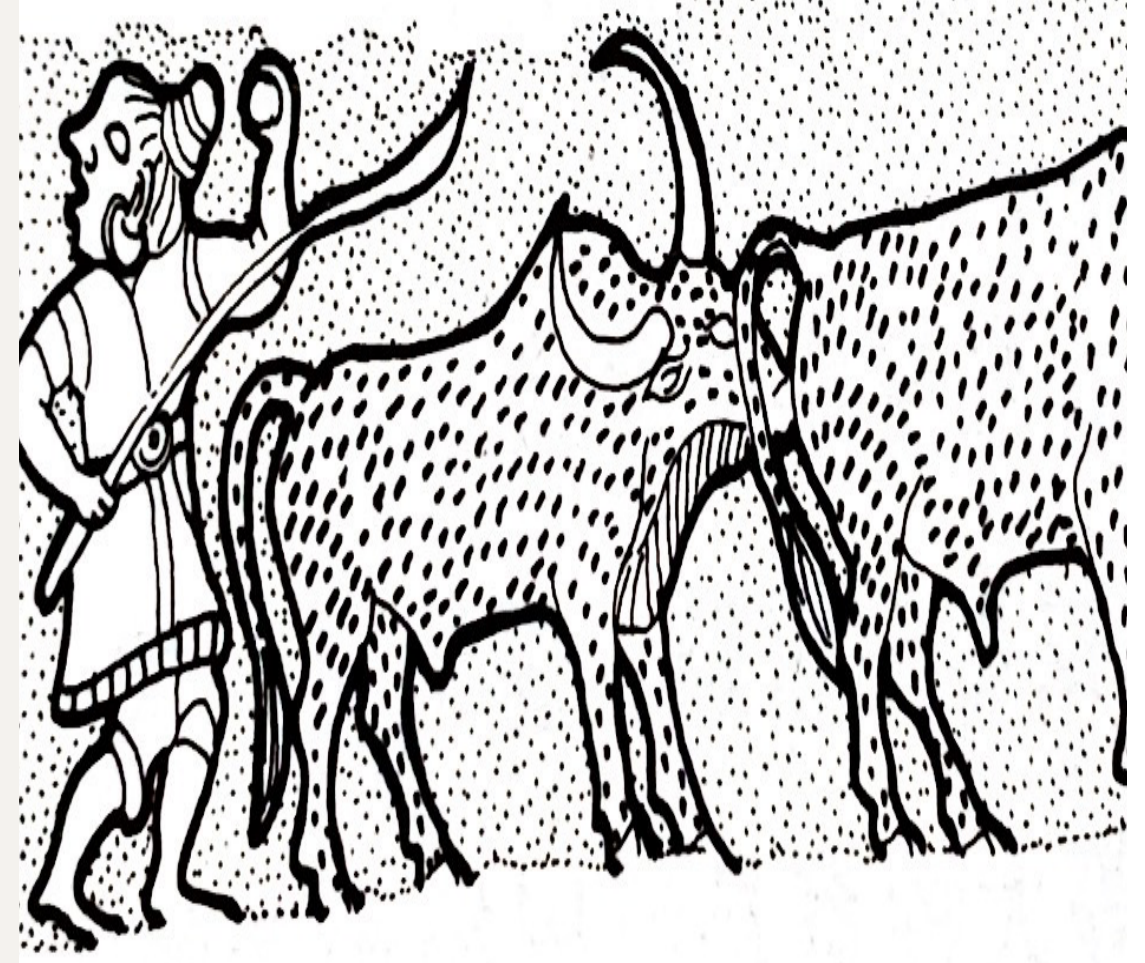

- The third is for foreign trade goods to other countries and local exchanges within Chinese provinces.

- The fourth is that cattle (and their heads, skulls) were used as symbols of wealth. The number of cattle within the family represented their status to the rest of Dian (Zhang, 2005).

Overall, Dian people relied on the lake heavily for food grow, then at the same time use the food to have a great farming.

figure 19. Dian people with cattle 


\section{AGRICULTURE IN CHINA}

Within China, farming was heavily influenced by environmental conditions, resulting in different regions farming different livestock and crops. Southern China grew a lot of rice but Northern China specialised in wheat due to the drought season (Zhang, 2005). The main animal husbandry in Southern China was mainly pig, cattle, chicken, and duck. Animal husbandry in the north is slightly behind if compared to the south that mainly have sheep and cattle. Both regions farmed cattle, but Southern China focused on smaller animals such as chickens, ducks and pigs. The North however, only raised sheep along with other cattle (Zhang, 2005).

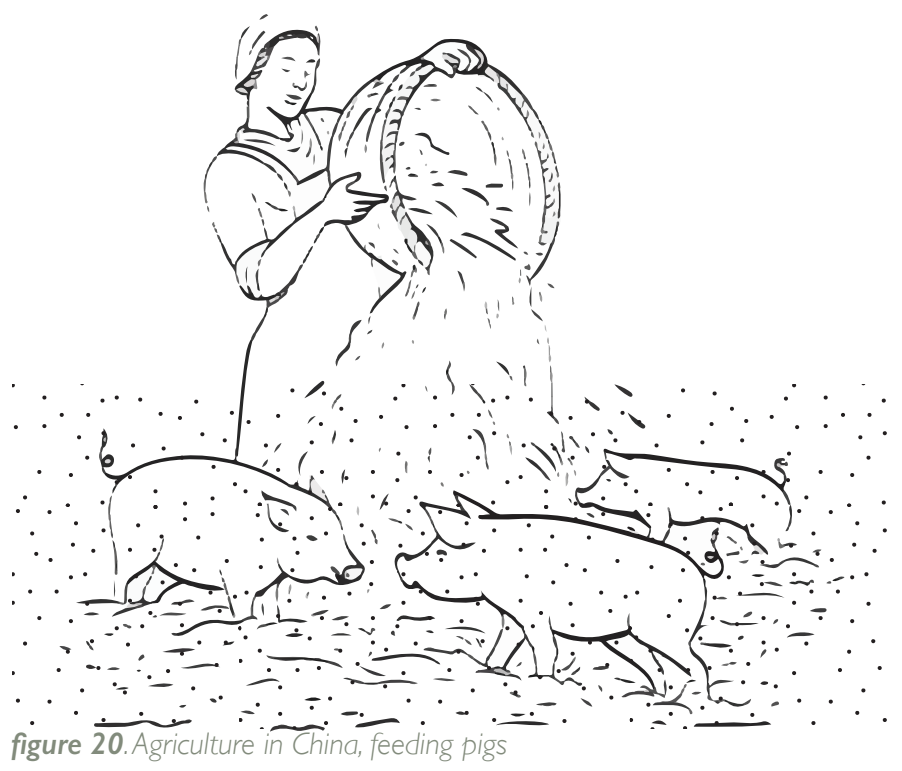




\section{ARCHITECTURE}

The environment conditions and the weather strongly influenced the Dian Architecture. As the forest cover the surrounding areas of lake Fuxian, Dian people can easily get timber materials from local. Yunnan is contained in the tropics, belonging to the subtropical monsoon climate which is humid and hot. The weather in Lake Fuxian has rainy season and dry season, rainy season has up to five months during a year(Fuxian Climate, 2020). The environment provided a good support for wild animals. According to these factors, the characteristic of Dian architecture are: easy to build, prevent attack by wild animals and suitable for warm weather.

Due to poisonous snakes and insects, Dian style buildings prevented wild animals from harming individuals via elevation manipulation (Zhang, 2005). There were mainly two types of buildings in Dian, column architecture and well-shaped buildings. Timber was preferred by the locals utilise these materials to build their houses Housing architecture predominantly contained two stories; where people occupied the second floor and fed to livestock on the first floor. Dian houses allowed Dian people feed their livestock conveniently. 
The remarkable thing of the Dian Architecture is the roof shape, resembling a traditional boat. This is visualised through the apex is much longer than the eaves. It is unusual within modern architecture. Reasoning around this stems from beliefs of exploration through nautical means being highly desirable (Zhang, 2005).

In response to this, Dian architecture prevents moisture collecting within the building, resulting in a cooling atmosphere (Zhang, 2005). Walls were typically made by weaving bamboo, leaving suitable thickness that aided in the ventilation of the entire room. A negative side affect to this however, is that the materials had low durability, meaning that there are no physical traces of these buildings left today (Zhang, 2005).

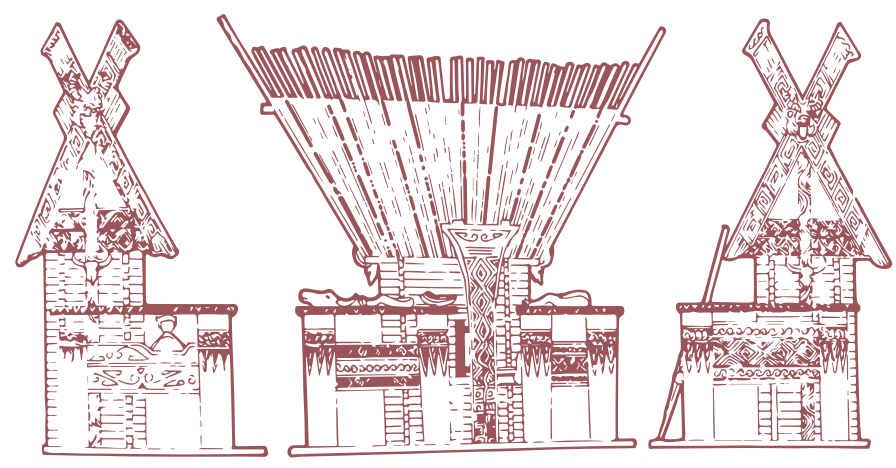

figure 2I. Dian Architecture 


\section{ARCHITECTURE IN CHINA}

The architecture of the Western Han Dynasty, which existed throughout all of China during the same era as the Dian Kingdom, can be divided into two types: royal and residential architecture. The royal architecture has more than three floors, however the residential building has only one floor(Zhang, 2005)(figure 22). Similarly, both the Royal buildings and residential architecture contain strong symmetrical aesthetics(Steinhardt, Nancy\& Shatzman, 2019).

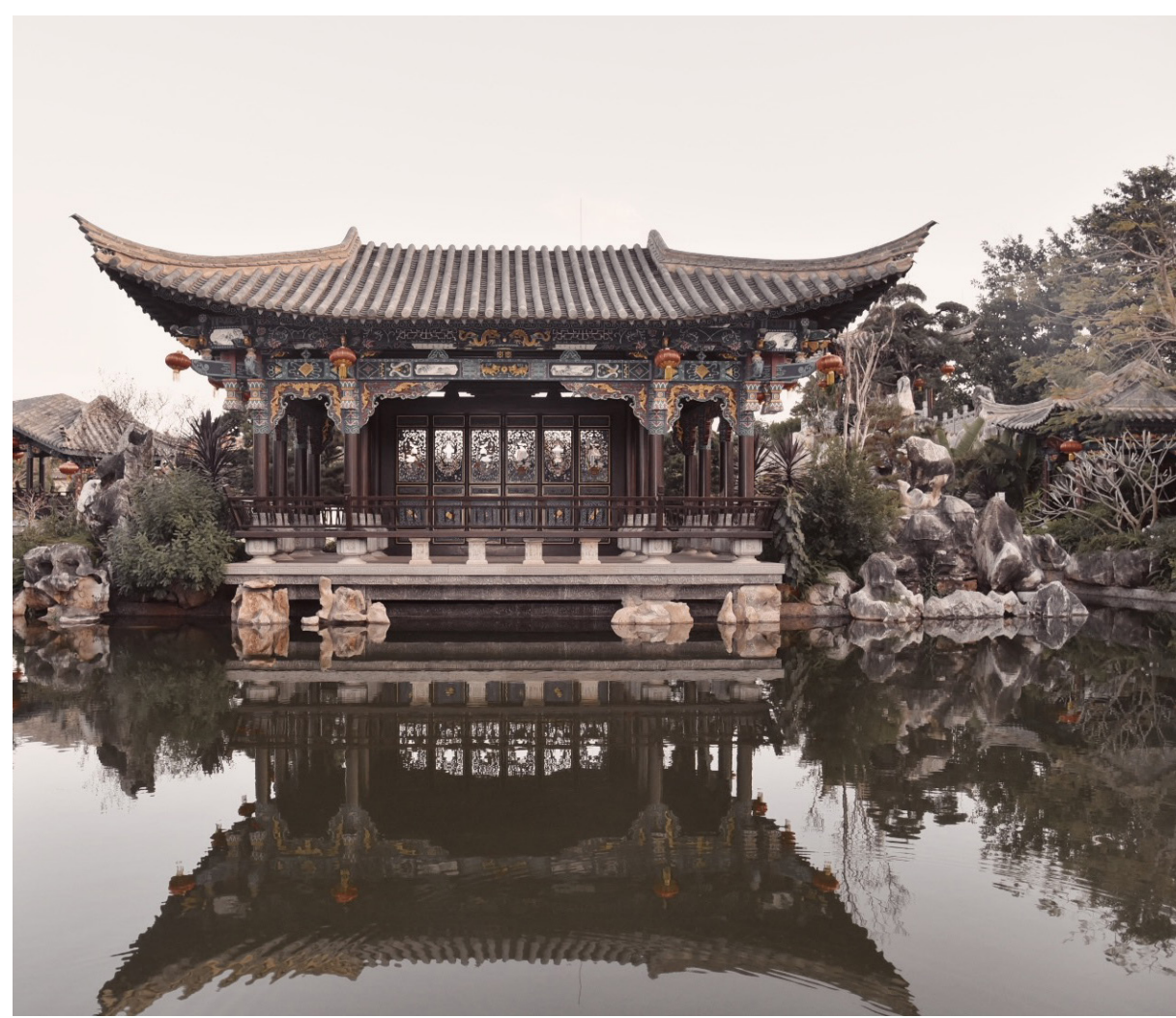

figure 22. Chinese Architecture

Chinese architecture has many connotations towards human form, and the home is no different. The base of the buildings is wide and supportive, reflecting how humans use their core and legs to balance against additional forces(Zhang, 2005).

Hence, the foundation is the most robust and longest section of the building(figure 23). The materials of Chinese architecture were mainly concrete, as it has great durability over time (Zhang, 2005). 


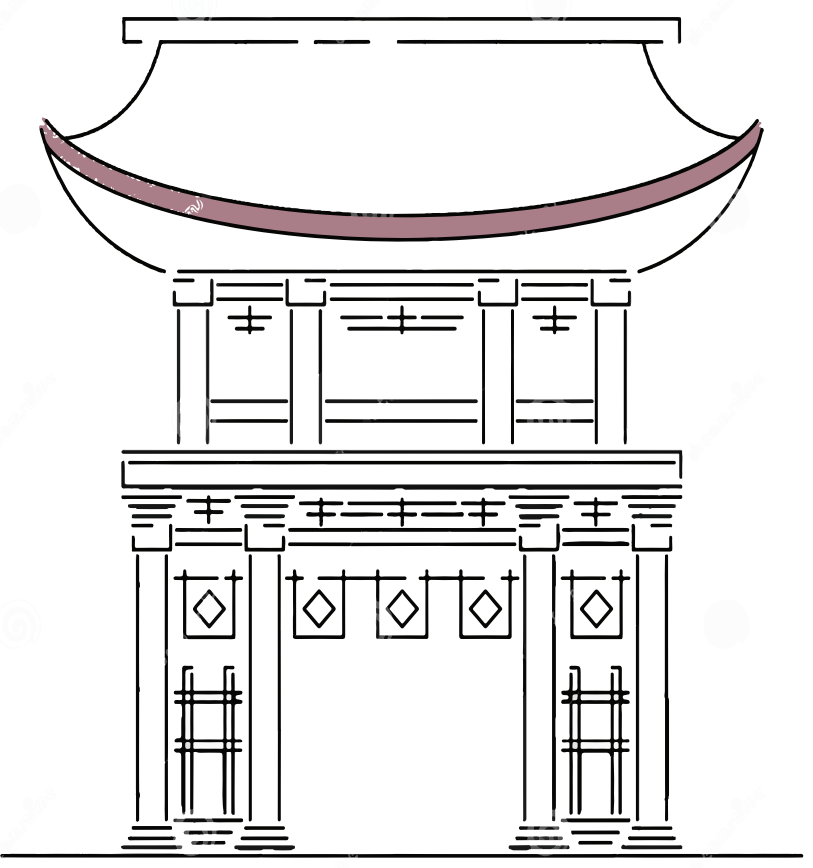

figure 23a. Chinese Royal Architecture in Han Dynasty

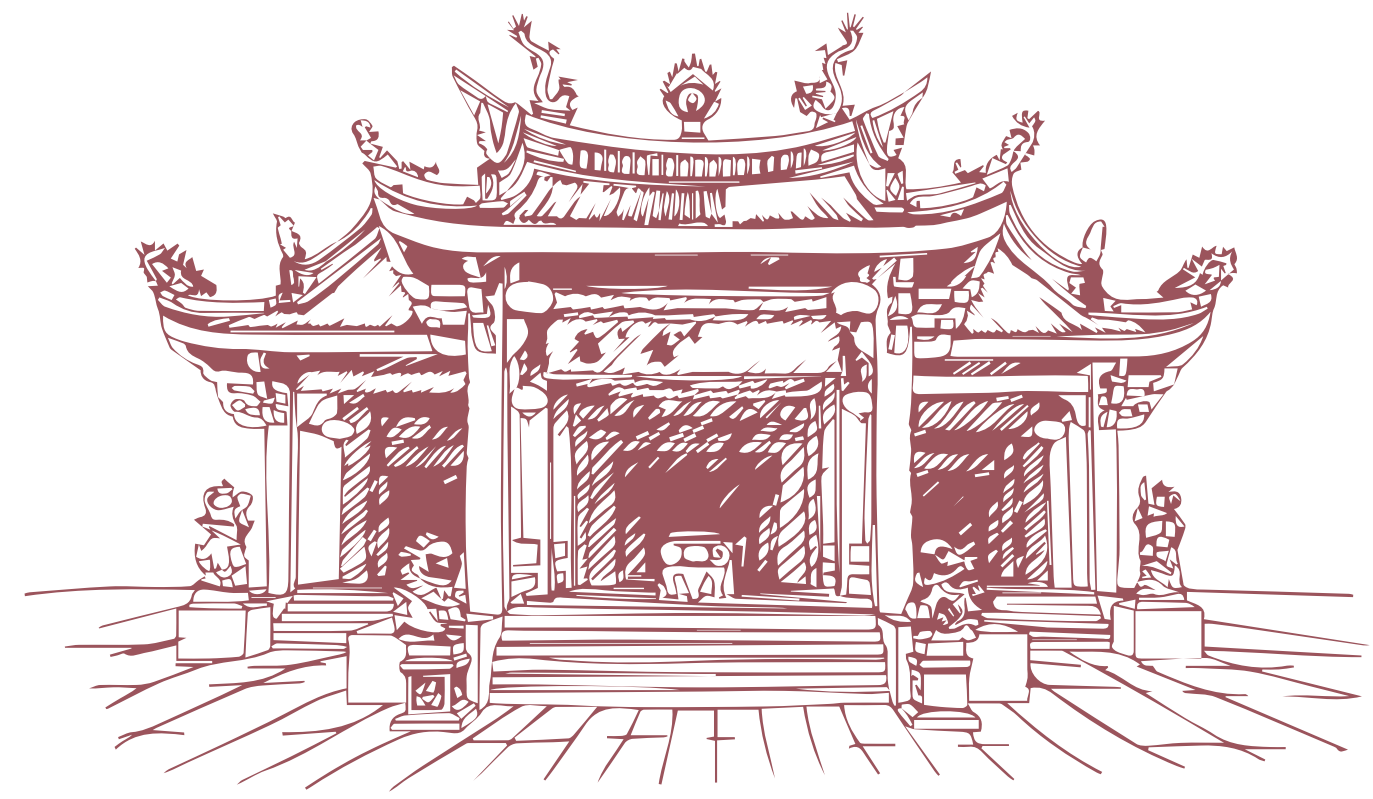

figure 23b. Traditional Chinese Architecture 


\section{COMMUNITIES}

PEOPLE

LAND 


\section{BRONZES}

Metallurgy is the pillar of Dian economy. The exploitation, smelting, and casting of non-ferrous metals is famous throughout China. The bronze drum is a type of bronze ware that was widely produced in the period of the Dian Kingdom, its main use was as a percussion instrument. The patterns upon the bronze drum record the story and history of Dian kingdom (Zhang, 2005). Despite its popularity, the production process of the bronze drum is very complicated (Zhang, 2005).
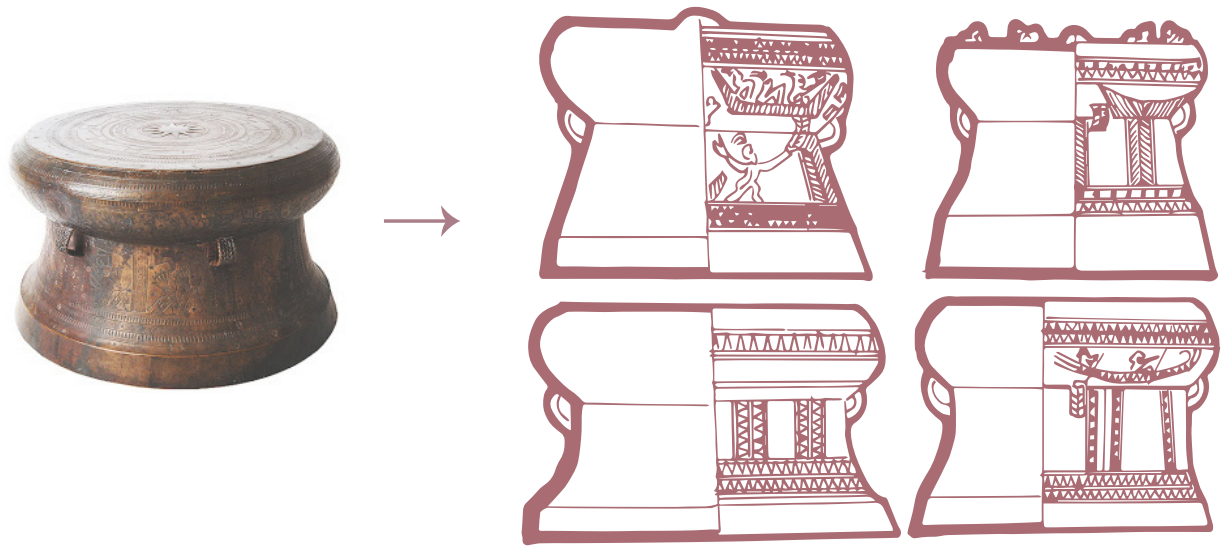

figure 25. Patterns on Dian Bronze 
At first, people need to make an internal model, and people may mix in some mud to make it hard. Secondly, people need to put it in a big model and fix the shape and bake it. Then, people began to draw patterns on them and cast them. Finally, people can install some accessories to make the bronze look impressive (Zhang, 2005).
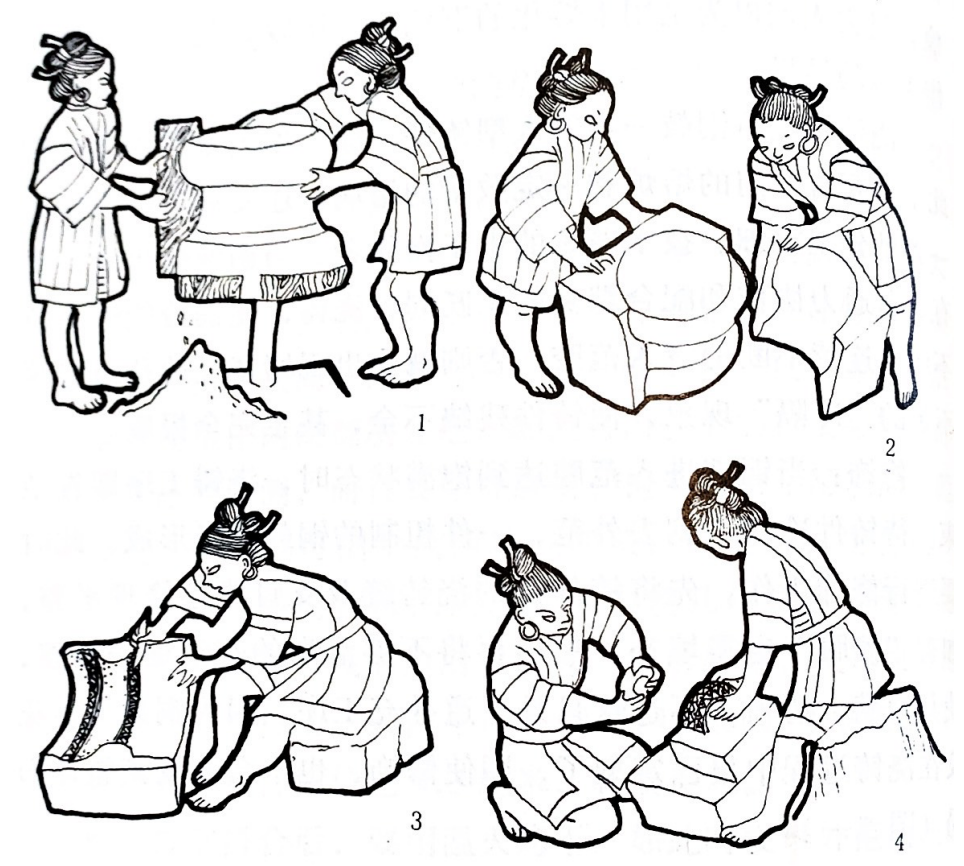

figure 26. Process of making bronzes 


\section{Belief \& Sacrifice}
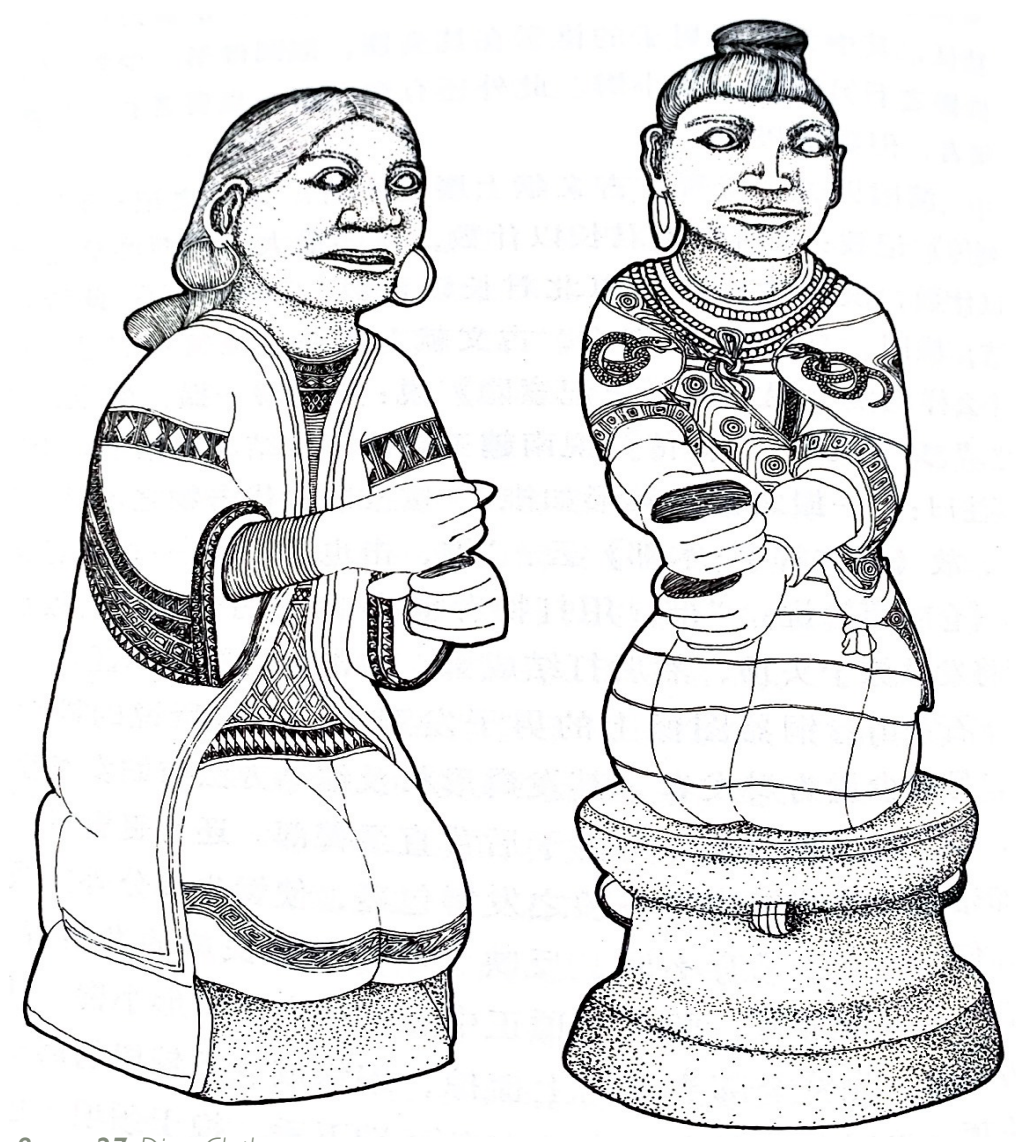

figure 27. Dian Clothes

Dian people generally believed in a more

nature-oriented religion, that is often called the worship of nature. In their view, people contacted everything in their production and life, such as mountains, rivers, thunderstorms, forests, rocks, wild animals, and their ancestors, all of them have potential and irresistible power. 
hese gods can bring good or bad luck directly or indirectly, and affect people's real-life at any time. In order to let the gods make them lucky and ask them to bestow and protect the world (Zhang, 2005). Dian people often had to carry out many complicated sacrificial ceremonies. Therefore, religious activities became a significant event in Dian ancient ethnic groups, affecting all production and life at that time. They often used some fierce animals, such as tigers and snakes, as sacrificial objects. The Dian residents will also sacrifice people and cattle so that the gods will not bring disaster to the human world.
However, in China, there is no sacrificial behaviour from the thoughts of Taoism and Confucianism(Zhang, 2002).

The agriculture, animal husbandry, and metallurgical industry in Dian were relatively developed compared with other regions in China, but the textile industry in Dian was relatively underdeveloped(Zhang,2005). The textile machinery was relatively simple. The raw materials for prevention and control were mainly hemp and kapok, and there was no silk fabric.

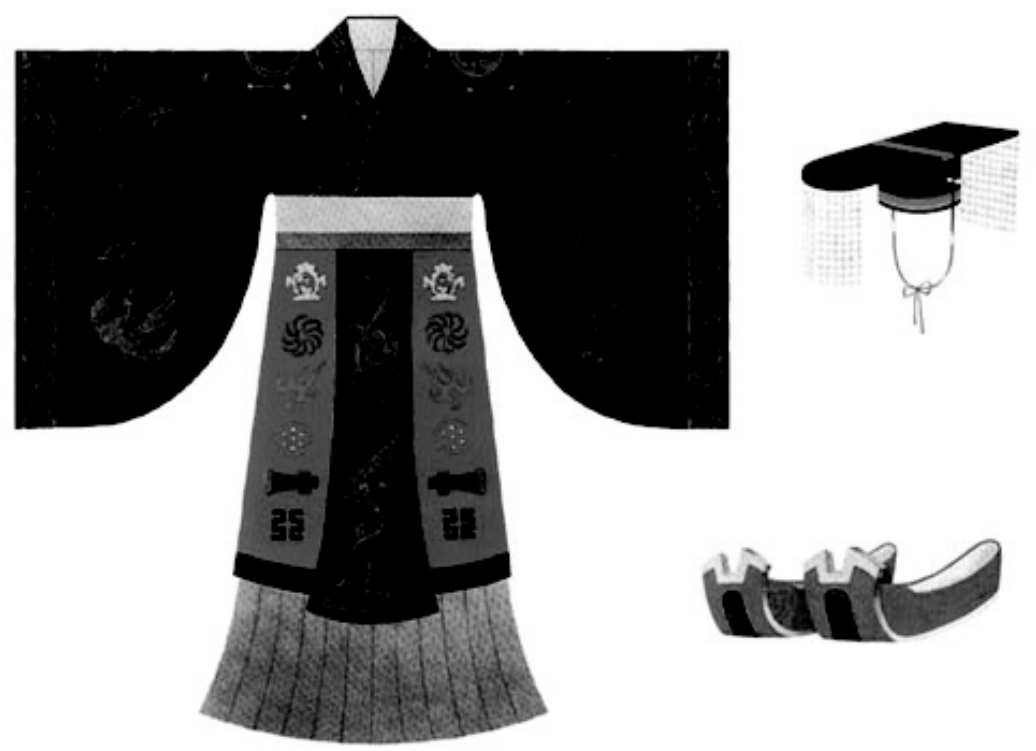

figure 28. Chinese Clothes during Dian period 


\section{Underwater Architecture}

Many years ago, there was a legend of an ancient city at the bottom of Fuxian Lake. This legend began when local people discovered stones with intricate patterns from the bottom of the lake. Later, a professional diver who grew up on the edge of Lake Fuxian in Yunnan Province, discovered a stone road at the lake's bottom during a dive. He vaguely felt that this was the legendary ancient city, so he carried out further research.

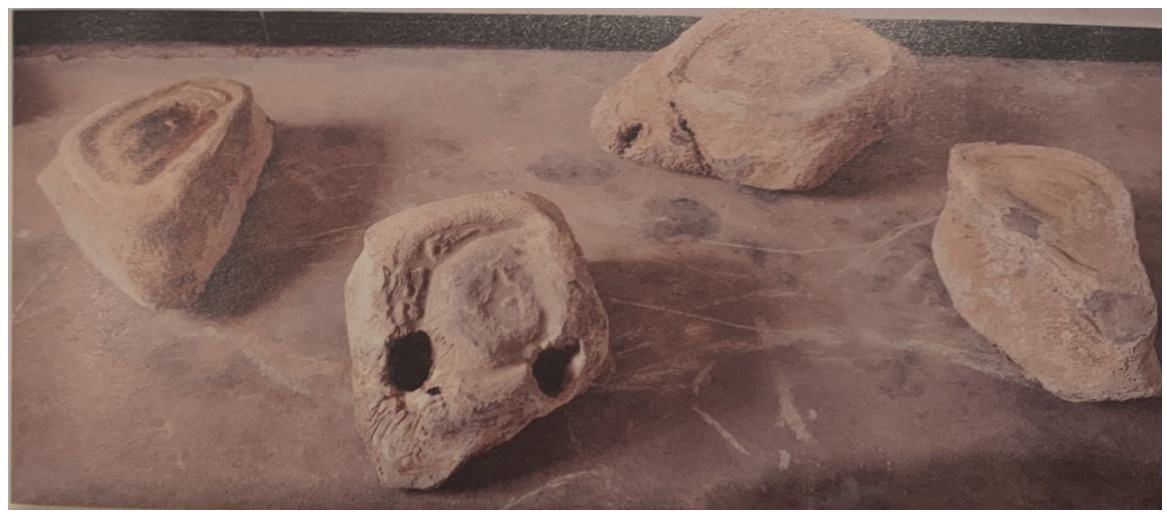

figure 29. Stones found by people, parts of underwater building 
Finally, in 200I, people found a large buildings in the bottom of the lake(Huang, 20I5). According to the sampling results, there are three different types of stone found on the underwater architecture(figure 29). The local Cambrian limestone and some surfaces of the building were similar to the fossils found in Maotian mountain. The other is red rock, which is the hardest stone that local people used to sharpen knives. The third stone is carbonate, which is a common limestone(Huang, 20I5).

The architecture found underwater is human-made and in average has 19 meters high and total 3000 square meters wide, in a complete terrace building. The base of the building is nearly 90 meters long and has five levels. Each terrace is elaborately engraved with symbols and words of sacrifice. Therefore, archaeologist speculates that this building was used by Dian people for sacrificial purposes(Huang, 20।5).

The building is about 1800 years old, during that period, Dian were managed by Chinese government, but able to maintain people traces of their culture. (Huang, 20/5).

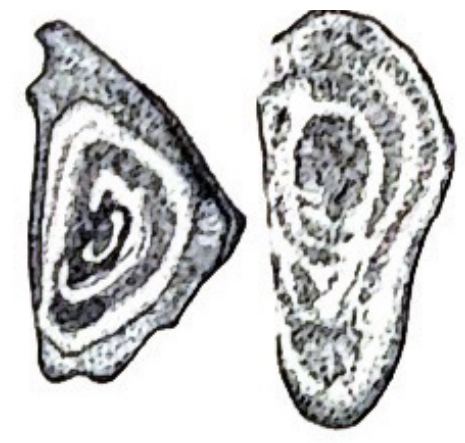

figure 30 . The pattern on the founding stones

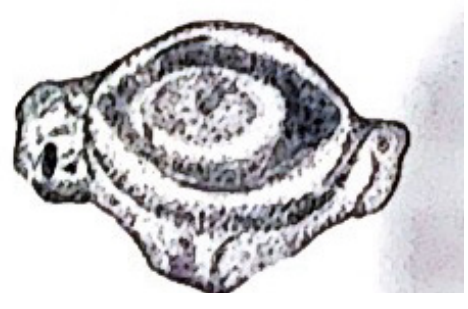


As for why the building was built in the water, there is no way to acquire a specific reason. However, most scientists acknowledge that Lake Fuxian is located in the earthquake fault zone where frequent earthquakes are known to happen. 1800 years ago, an earthquake occured, and a large amount of sand and rock entered the lake. This could have caused the lake's water level to rise and submerge the surrounding architecture on the lake's edge.

Until today, the public have not been given a specific location within the lake of this structure, in order to protect the architecture. On the other hand, the underwater building attracted more visitors to come to Lake Fuxian. According to Huang(20I5), after a documentary called "Exploring the underwater architecture in Lake Fuxian" released, more visitors visit Lake Fuxian. After local government analysis, the architecture attracted at least one million visitors to the site(Huang, 20|5). However, individuals with a professional freshwater diving license can dive to see the underwater structure for themselves. So, most of the locals and visitors could not see the underwater structure.

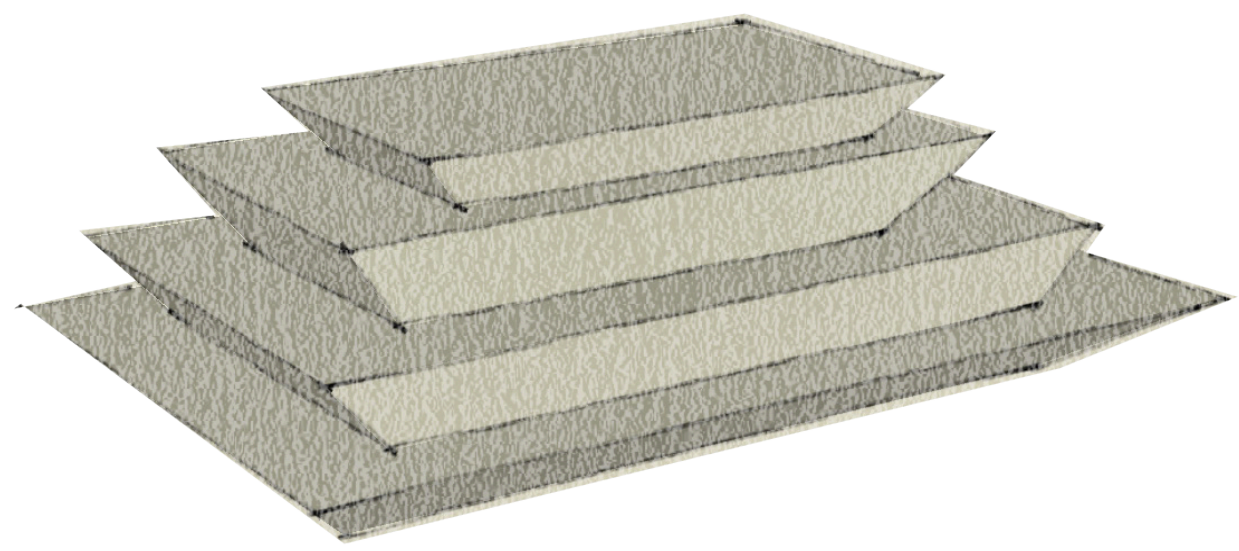

figure $3 \mathbf{I}$. The shape of the underwater building 
SUMMARY

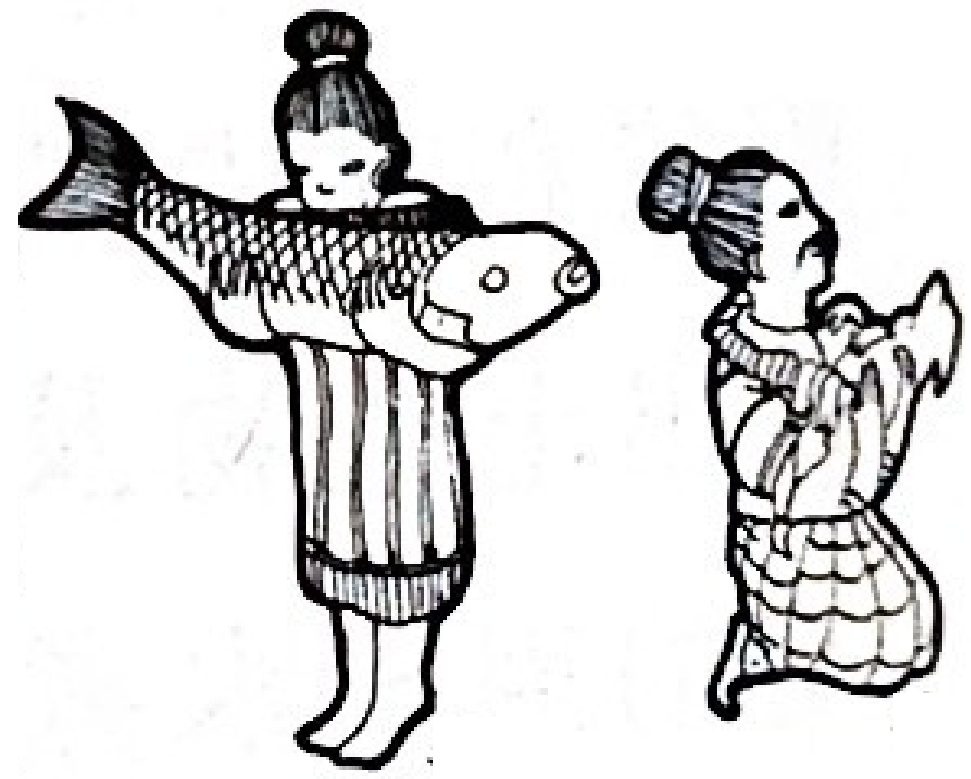

figure 32. Dian people eat fish

To conclude, Dian people settled along the shores of this Lake around 2000 years ago. However, after long years of conquests and land warfare, the kingdom was left uninhabited, with only small remnants of civilization left today. Lake Fuxian has a unique history during a different period. The name of the district is meaningful, and it identifies the Lake is a place that has interesting history and culture. Then, the fossils found on the site have recorded that palaeontology has lived in the area during the Cambrian period(54I million years ago - 48 I million years ago) (Huang, 20|2).

44 
The climate condition affects local agriculture, and the environment is good for planting rice. Therefore, the main food for Dian people is rice, and the meat source is mainly from livestock and poultry fed by themselves. Besides, Dian people liked to get wild blackberries from the local forest, and they ate fish that catch by a singular tool(figure 33) from Lake Fuxian. The Dian Architecture materials are based on the local landcover, and the functions of the architecture illustrate the local environment. Furthermore, due to the pollution from agriculture, Chinese government does not allow any agriculture around the Lake (KunmingNews, 20/9). Nowadays, people cannot see any agriculture activities on the Lake, and they do not know the unique agriculture in Dian period in Lake Fuxian. In addition, because Dian Architecture is made by ow durability--timber, after 2000 years, there are no Dian Architecture left today.

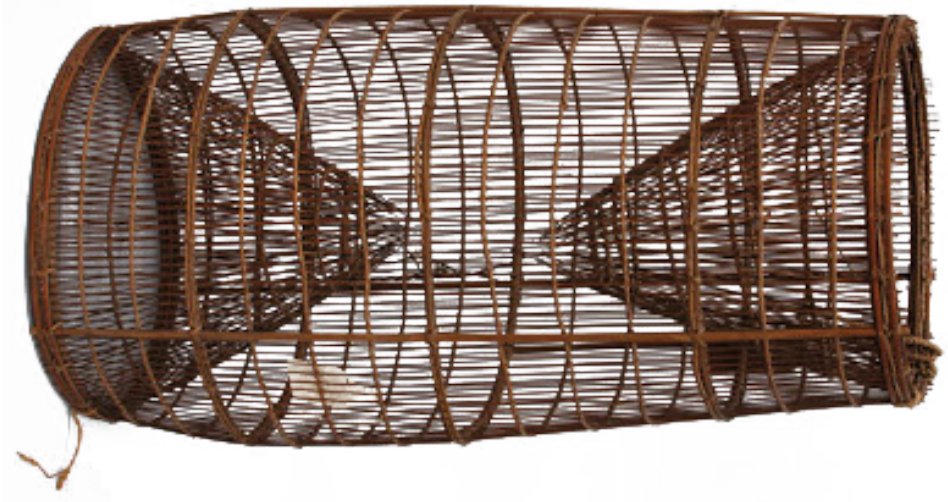

figure 33. The fishing tool made by Dian people 
The bronzes made by Dian people have recorded distinctive Dian culture. Unlike Chinese people, Dian people believed in a more nature-oriented religion, and they often had sacrifice activities. The architecture found underwater recorded the culture 1800 years ago(Late Dian period) (Huang, 20I5). The pattern on the building has illustrated under the management by China; Dian people started to believe "Yin" and "Yang" and Chinese "Fenghsui" in 1800 years ago (Huang, 2015 )(figure 34).

Overall, the architecture of Dian and the culture of Dian is different from Chinese culture (figure 35). To highlight the local identity of Lake Fuxian, it is important to use Landscape Architecture to display the culture and history of Lake Fuxian.
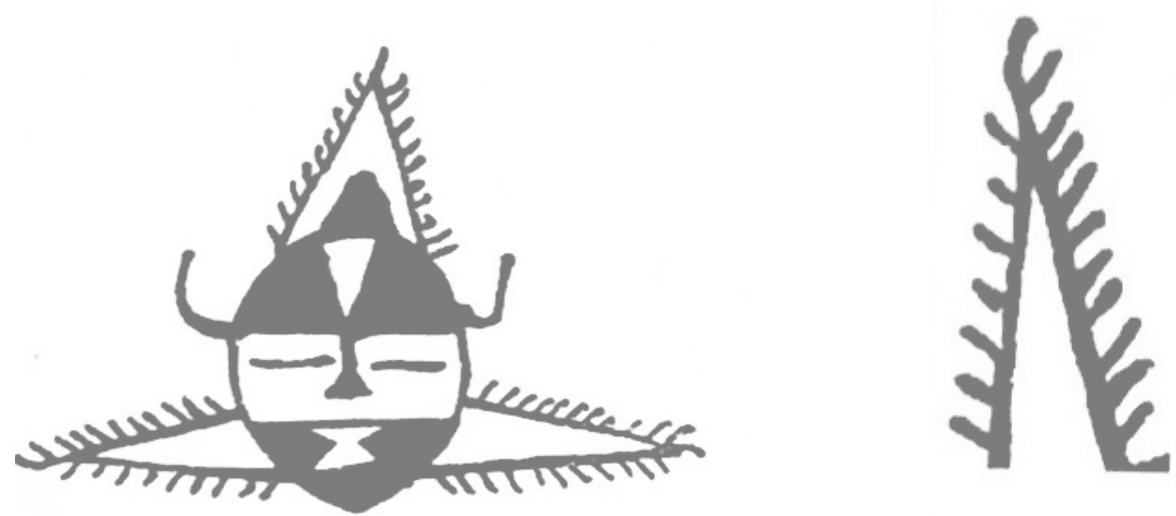

figure 34. Pattern found from the underwater Architecture 
DIAN KINGDOM
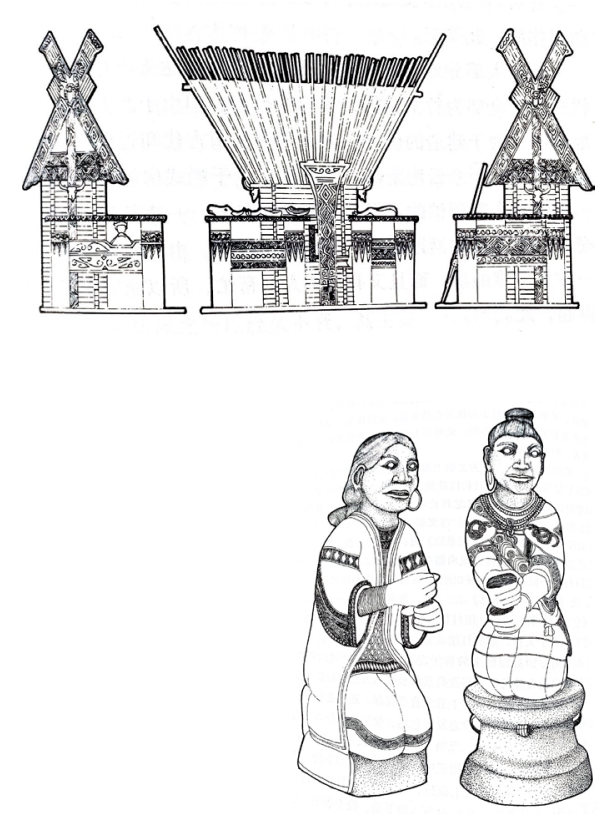

CLOTHING

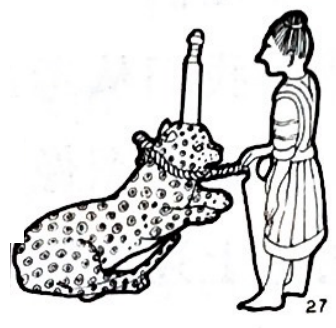

CULTURE

BELIEF

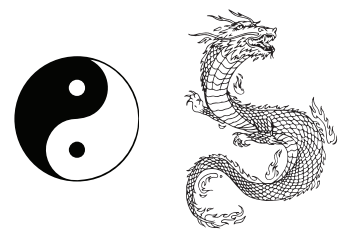

ANCIENT CHINA
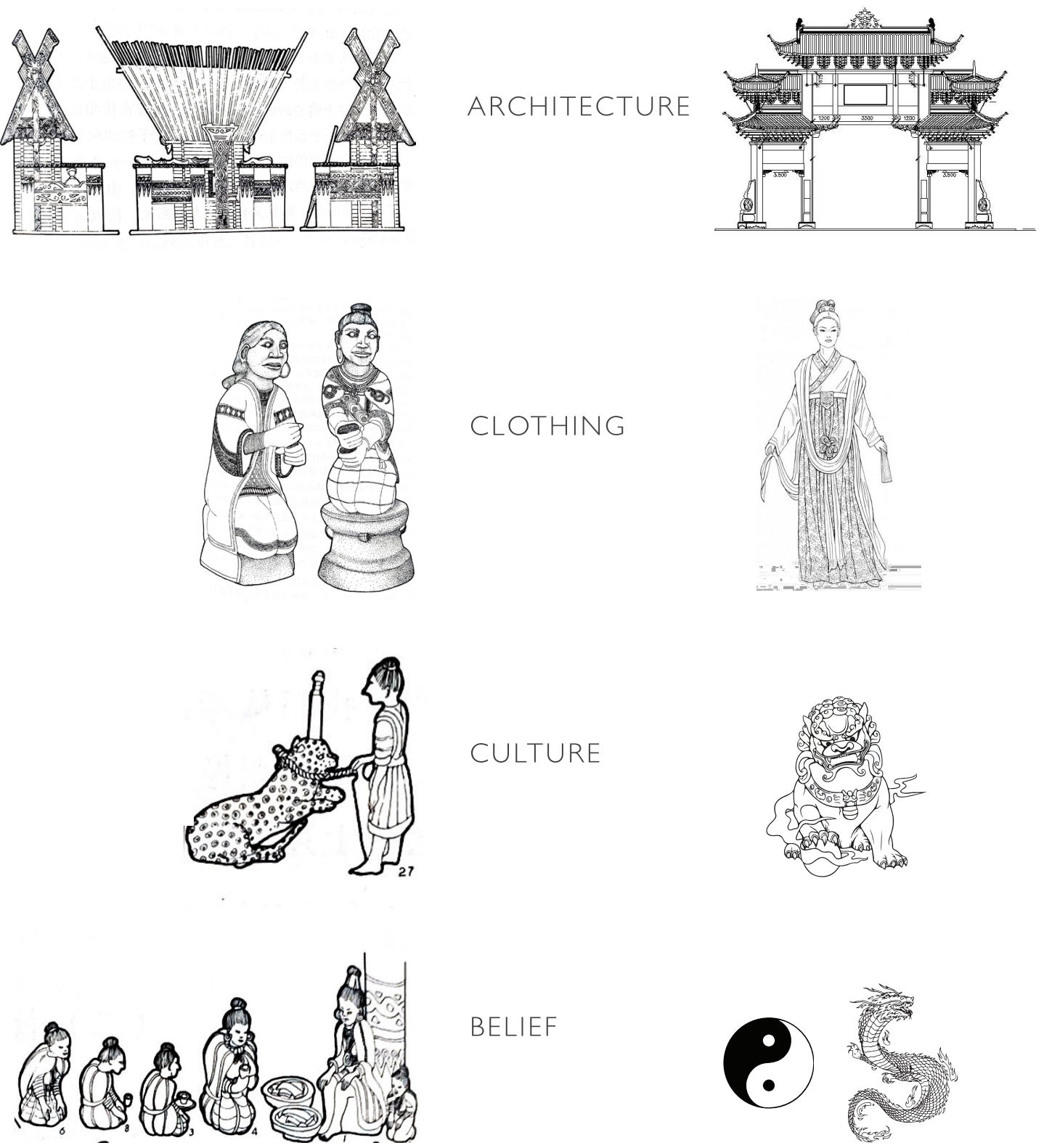

figure 35. Comparison between Dian Kingdom and ancient China 


\section{PROBLEM STATEMENT}

There is a group of non-Chinese metal working tribes called Dian Kingdom settled along the shores of Lake Fuxian around 2000 years ago. Dian people settled along the lake for about 300 years, and remained some relics that allow people to know their culture (Zhang, 2005), he unearthed relics found around the lake recorded the detailed history and Dian culture in Lake Fuxian.
Archaeologists found that Dian architecture is unique globally, and it is totally different from traditional Chinese Architecture (Zhang, 2005). Also, Dian culture is special and have many differences from Chinese culture. However, there are small remnants of civilization left today, and people could not find local Dian features from the physical Landscape in Lake Fuxian.

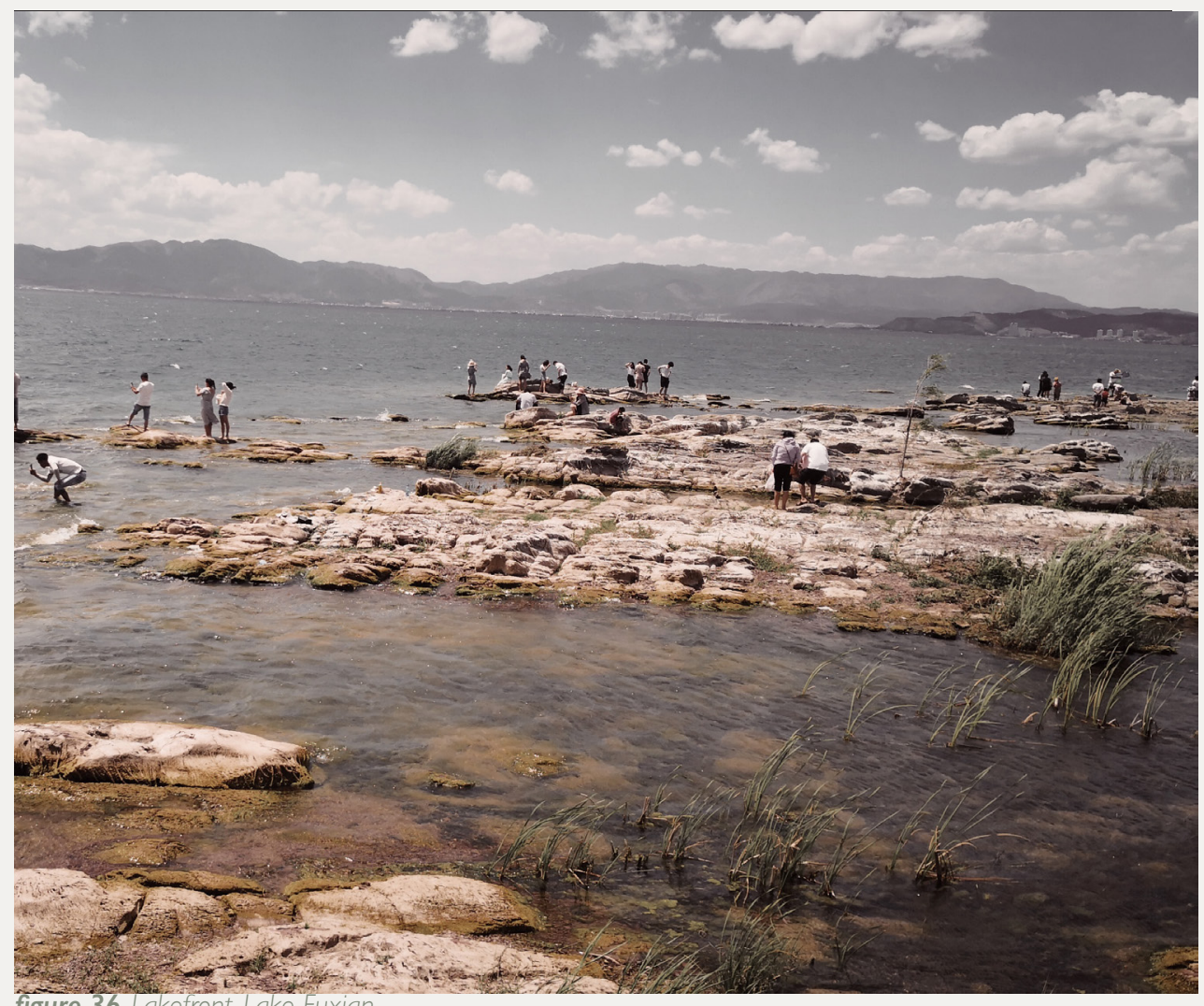

figure 36. Lakefront, Lake Fuxian 


\section{AIMS \& OBJECTIIVES}

-To explore design methodologies associated with landscape narratives

- To reveal the hidden narratives based on the history and culture of Lake Fuxian

- To discuss the importance of the use of new technologies in the design of Lake Fuxian

- To create landscape architectural design solutions that connect both the physical landscape and the augmented layers of information to provide visitors an educational journey in Lake Fuxian 


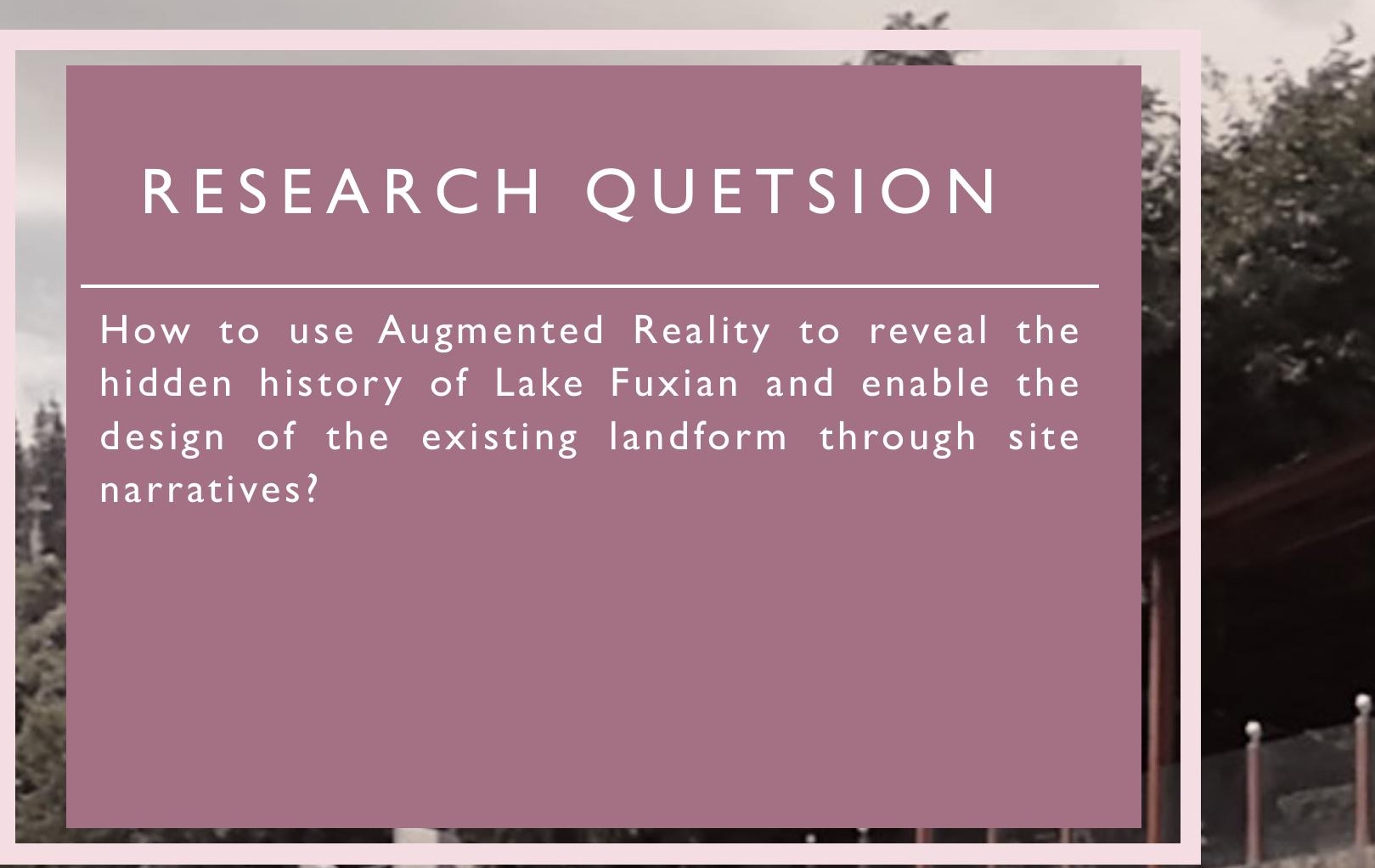

Why
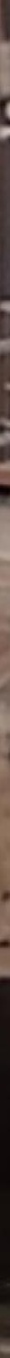


\section{Site Overview \\ Research Question \\ History \\ +Objective}

Climate

Culture

Introduction

Theory

Design Approach

Site Analysis

\section{Reflection + Conclusion}

Design

Connect

Reflect 


\section{nt Review}

ardens

\section{Design Methods}

Site Analysis

Wide Context

Closer Scale

I 4 sites

\section{Chosen Sites}

2 chosen Sites

$\downarrow$

Preliminary Testing

Design

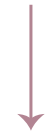

Developed Design

Detail design for zoom sites 


\section{LITERATURE REVIEW

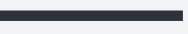


The Literature study includes three aspects which are: New Technology, Narrative Landscape and Chinese Philosophy.

The New Technology explored how technology can enhance Landscape design and two similar technologies Virtual Reality and Augmented Reality can assist in revealing hidden narratives of the land. After comparing these two technologies, one of the technology was picked for future study.

Because the design needs to address the narratives of a space history and culture in Lake Fuxian. The section titled "Landscape Narratives" addresses the importance of the narrative design, and how to design a space.

Due to the location of the lake in China, as wel the 1800 years of management by the Chinese government, it is important to compare and contrast the similarities and differences between Dian and Chinese culture. Therefore, Chinese philosophy is important to understand the design of traditional gardens and the identity suitable design criteria for designing a space that contains both aspects of Dian and Chinese culture.

Overall, the literature study will integrate with the precedent study and the method to create a Landscape narrative that can be used it in the design of Lake Fuxian. 


\section{LITERATURE STRUCTURE}

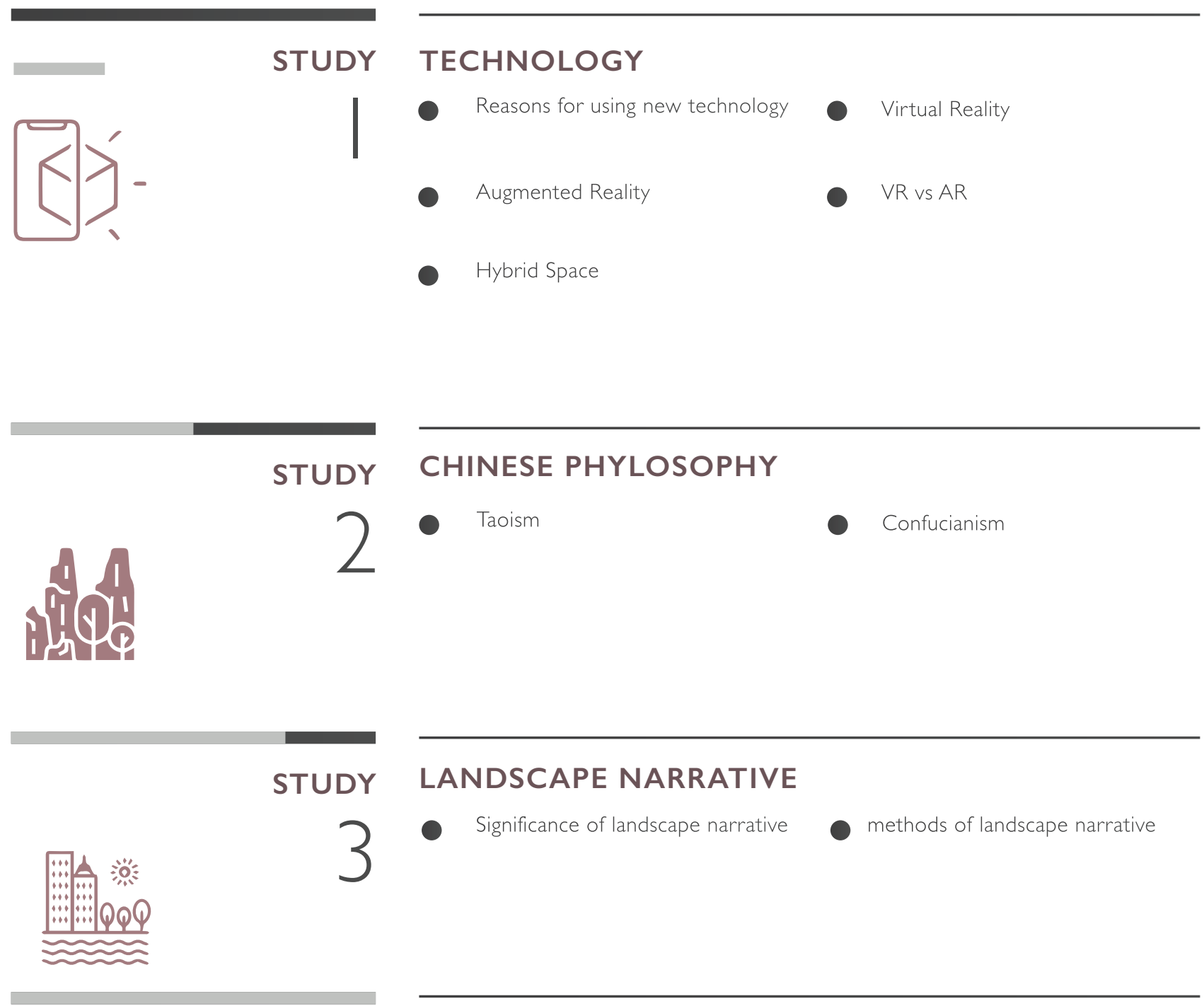




\section{TECHNOLOGY}

This chapter will explain the reason why new technology can enhance landscape design. Then, it discovers what types of technology can be used in landscape design. At the same time, compare the new technologies and pick more appropriate technology for this design research.

It is a well-known fact that as the modern world advances, so too does the technological world, at an equal, if not at a more rigorous pace. There are many facets of technology which are constantly developing, from vehicular transportation to the world wide web and handheld devices. Merging both the internet and handheld devices, visually

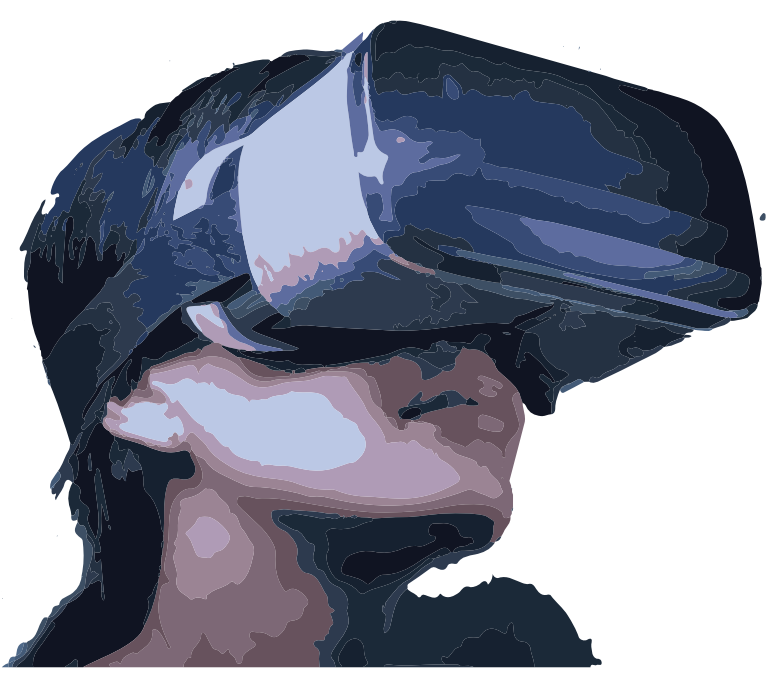

figure 39. people with VR altering technology has made an enormous growth (Walliss \& Rahmann's, 2016).

Virtual Reality (VR) is a compelling computer application that allows humans to interface and interact with computer-generated environments (Grigore \& Philippe, 2003). Virtual Reality is based on a digital world that mimics real-life by engageing and emphasising all of the senses (Grigore \& Philippe, 2003). VR can be used in clinical studies, commercial selling and different situations. For example, doctors use VR to their patients who is different to go outdoor space (Riva et al, 2016). Recently, people increasing use of VR in behavioral health particularly in the treatment of anxiety disorders, because it can bring people to a Virtual World, and relief their anxiety (Riva et al, 2016). In China, there is an App support to the people who want to rent a house using $V R$ to see the house instead of coming physically to the house's site (Beike, 2020).

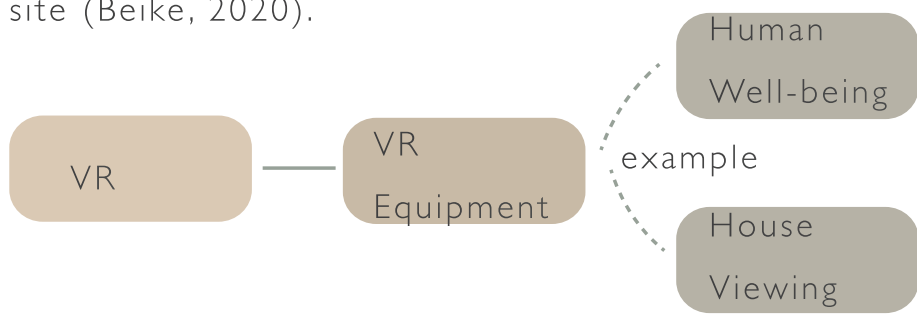

figure 40. Virtual Reality Diagram 
Unlike VR, Augmented Reality $(A R)$ has to be established in the physical world. AR was created in 1990, the function of $A R$ is to allow the electric devices to link to the digital realm(Alexander, 2017). Then, it widely used in historical building reconstruction to illustrate some disappeared heritage by using an application on smart devices(Noh, 2009). It can continuously add layers to the physical world to let users have more interactives with the physical world(Alexander, 2017). The AR relies heavily on the individuals personal needs in each location (Noh, 2009).

Similar with $V R, A R$ also can be used in the treatment by doctors(Riva et al, 2016). It can be a learning tool that improves people's concentration and motivation,

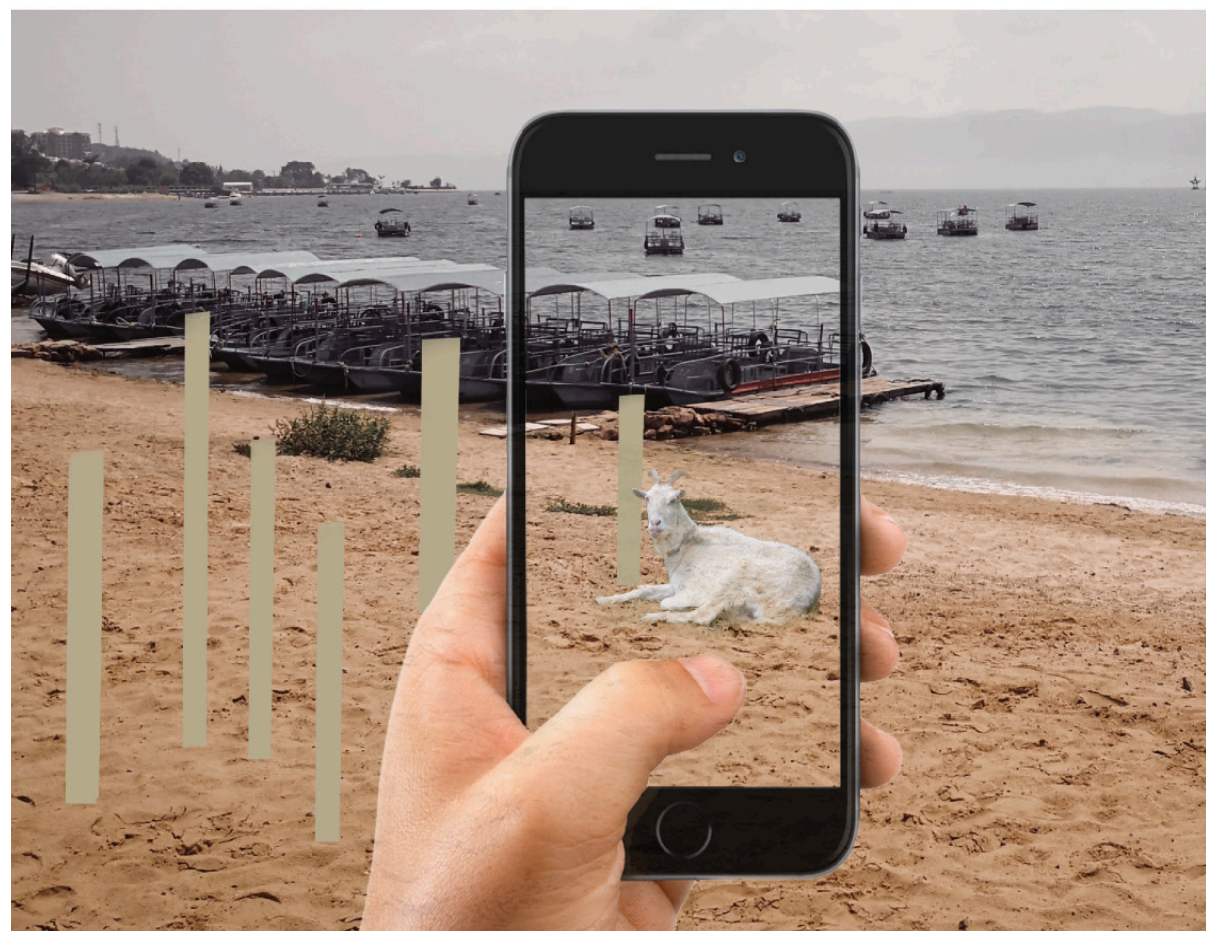

figure 4I. potential AR technology used in Lake Fuxian

and used in treatment of phobias(Riva et al, 2016). According to Riva et al (2016, p6) "AR can be used in the actual places where the subject encounter his difficulties, facilitating the transfer of the acquired skills to the real world." Additionally, AR has been a tool to use in toys for early childhood education, it can shape children's experience and enrich their imagination (Yilmaz, 20।6).

Walliss and Rahmann's

$(20 \mid 6)$ discovered the limitations and opportunities of both new technologies and Landscape Architecture. They discussed how new technology allows Landscape Architecture to be visible and conceptual as it expands on what originally could only be seen on paper (de Souza e Silva,2006) It is a tool that connects the invented world to a physical location, whether the digital prototype's design exists in that time and space (Alexander, 2017). 


\section{- Hybrid Space}

According to de Souza e Silva (2006), "Hybrid space" is described as the borders between physical and digital spaces by using digital technology and devices. They discuss a positive example of Augmented Reality, where a hybrid space was created in Japan. This example is a location-based game that creates circumstantial possibilities for people to navigate site, through the act of finding wildlife. It is an excellent way to encourage continual usage, as the hybrid space adapts to local time changes, as the wildlife follows realistic habitual movements (de Souza e Silva ,2006). The game relies on individuals being in the right location at the right time of day, to maximise the amount of information being uncovered (de Souza e Silva ,2006). There is also an educational appeal to the design, as it can be used by the younger generation to learn about behaviour and movements of specific species throughout a city (de Souza e Silva,2006).
Overall, due to easy accessibility and the wealth of information available, this design can be classified as a successful use and adaption of AR technology.

Stuart (20|2) expands that AR is essential for the archaeology vocation, in that it allows parts of the real world and known elements of the past to be actualised within one space.

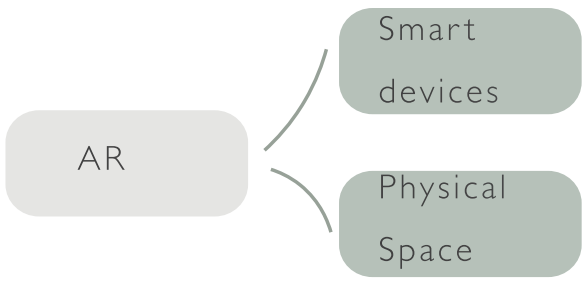

figure 42. Augmented Reality diagram
This allows for people to experience and explore the entire history of a site when only small pieces are physically present.

examples

Children

Education

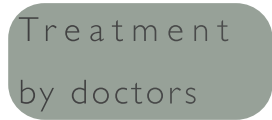

Building

Reconstruction

figure 43. hybrid space

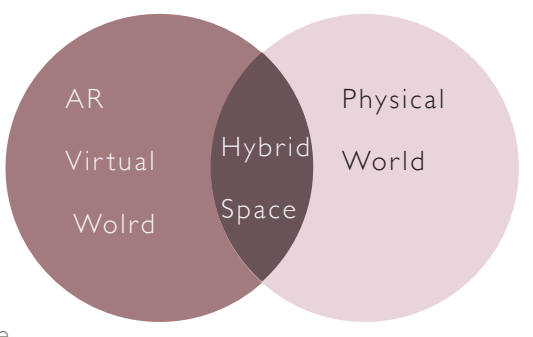




\section{CHINESE PHILOSOPHY}

To a certain extent, traditional Chinese landscapes reflect many aspects of everyday life, such as their cultural world view, philosophy, artistic spirit, and personality. This is reflected in the style, layout, and aesthetic taste of the gardens (Chan, 2008). These ancient designs were mainly influenced by Chinese Taoism and Confucian beliefs ( $\mathrm{Xu}$, et al., 2018). This research will study how the Taoism and Confucian religion has influenced Chinese Landscape Architecture and the possible potentials for inclusion within the design phase of this thesis.
T Taoism has a long and impactful history

A throughout China, dating back to its origin O in 4th century $\operatorname{BCE}(X u$, et al., 20l8). S Taoism has two famous creators: Laozi and M Zhuangzi (Xu, et al., 20l8). They believe that the world and Universe is a large organic system. The system with endless vitality is called Tao and is considered the ultimate foundation of the universe ( $\mathrm{Xu}$, et al., 20/8). In Taoism, Tao is transcend and impregnates everything ( $\mathrm{Xu}$, et al., 2018). Zhuangzi offers this opinion within his subject law of nature, which specifically states "Heaven and Man Are United as One"

In China, many architects and landscape

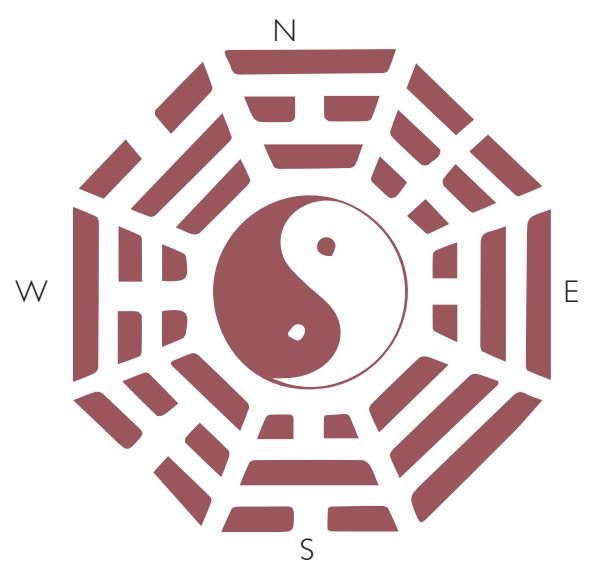

figure 44. Fengshui Universe in Taoism version 
designers need to follow the concept of Taoism. However, these are simplified into three categories;

I)Fengshui(figure 44) (comprising ancient arts of necromancy, astrology and medicine) it believes there is an "Invisible force" after planning a space or an architecture with the universe, earth and humanity. It highlights the significance of nature to human affairs, and humans can pursue life and values from the interaction with nature (Chan, 2008).

2)Yin Yang(figure 45)(negative or positive, opposing principles in nature) It is described as two contrary forces interrelate to one another (Chan, 2008)

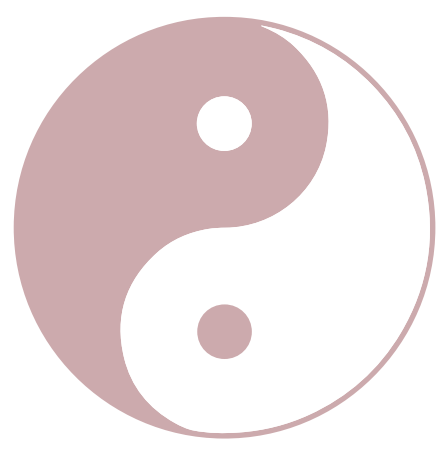

figure 40. Yin and Yang
3) Wu Xing(figuer 46) (includes the five elements, metal, wood, water, fire and earth) (Qiao, 20I5). This order of presentation is known as the "Days of Week" sequence. The five elements allow for interaction with each other, as well as having individualised space (Zai, 20I5). In traditional Chinese gardens, designers always utilise different colours and materials to represent the five elements within Taoism, such as

Earth,(Incense), yellow, center, up and down Wood (Spring Wind), green.

Fire (Summer Dew)red, Metal(Fall Sounds) white,

Water (Winter Sunshine) black/blue

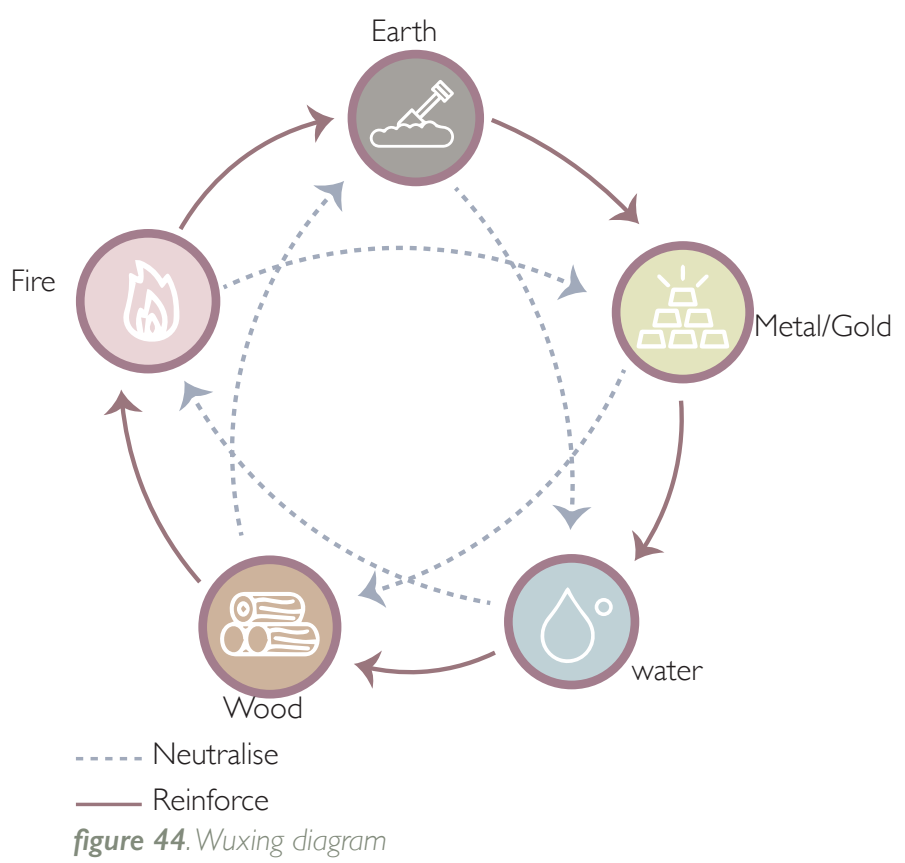


One of the main design elements within Tao inspired classified Chinese gardens, is the illusion towards infinite space (Chan, 2008). The landscape's infinite space is applied through the existing structures and aims to allow individuals to experience the feeling of the landscape and spiritual transcendence (Chan, 2008). For example, people put walls to design a maze in a small space, and make people feeling the space is infinite (figuer 45) (Wenku, 2013).
Appling this, there is a lot of potential within the design phase, to include traditional roots. Taoism will play a fundamental part. Fengshui, Yin Yang and Wu Xing will act as design principles for all future design concepts.

It is important to include Taoism within this thesis as a design strategies in the design of a Chinese public park.

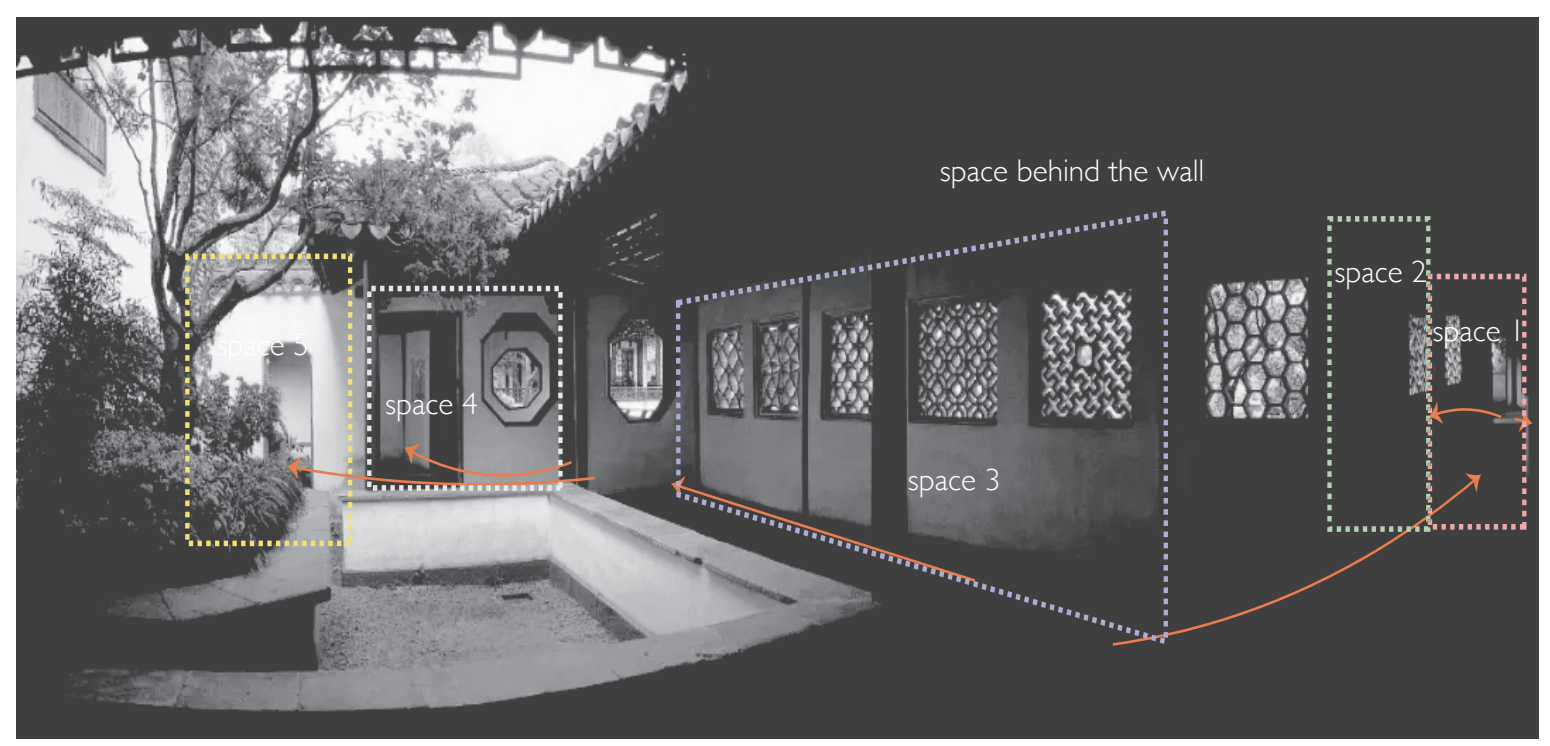

figure 45. use walls to create interesting movements and divide a big space into several small spaces 


\section{CONFUCIANISM}

Confucianism was established during the 500 BCE, and was called as such, as it was founded by a man called Confucian (Chan, 2008). According to $\mathrm{Xu}$ et al. (2018, P4) "The core of Confucian thinking is "Ren"" which can be translated to humanity. Humans as a part of the universe and should respect heaven and love earth ((Xu, et al., 20/8). He believes everything needs to be based on humans' "Wushi" (five senses) which are touch, smell, taste, sight and sound described (Xu, et al., 20।8).

Therefore, following Confucian beliefs "advocating nature and learning from nature" has become a principle followed by Chinese traditional garden design ( $\mathrm{Xu}$, et al., 2018, P 8). Under the influence of this

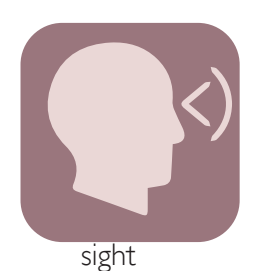

sight

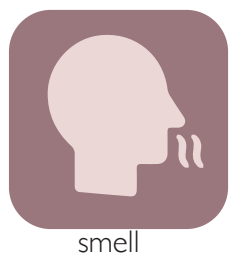

smell

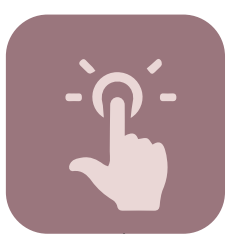

touch

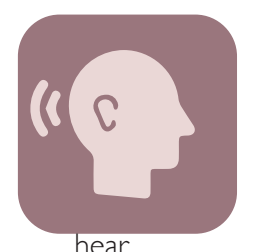

hear

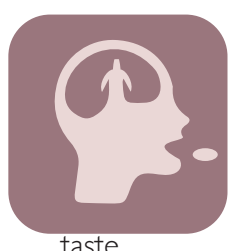

taste figure 46. Chinese "Wushi" ( five senses) sight, smell, touch, hear and taste idea, traditional Chinese gardens organically integrate material components with natural conditions, unifying both natural and artificial aesthetics (Qiao, 20|4).

The planting selection within a Chinese gardens is heavily influenced by Confucian philosophy. The Confucian idea of comparing morality has influenced the theme of traditional Chinese gardens, such as paying special attention to the morality, or immortality, of physical elements of the design (Qiao, 20l4). The flora within a Chinese garden can give people spiritual strength and indicate characteristics similar to people's ideological character $(X u$, et al., 2018). People often use flowers and trees to represent people's idealistic sentiment and narrative of the site (Chan, 2008) For example,(figuer 48 at next page) in Chinese culture, bamboo describes as a strong man because it can grow well in a bad weather condition. In addition, people often describe bamboo is a handsome man, because of its' slender shape (Chan, 2008). 
Traditionally, plants including pine, bamboo, and plums and are frequently titled the "three friends of winter" in China mortality. For thousands of years, these plants have been praised for their noble quality. developing into symbols of strength, longevity, and integrity, often becoming the most artistic feature within the park.

Therefore, they are respected and praised by people as they represent human's great strength and positive work attitude. Landscape designers in China often use the meaning of plants within a narrative, to illustrate the landscape's story. However, this part of the design can be undervalued and misunderstood if visitors do not poses this knowledge.

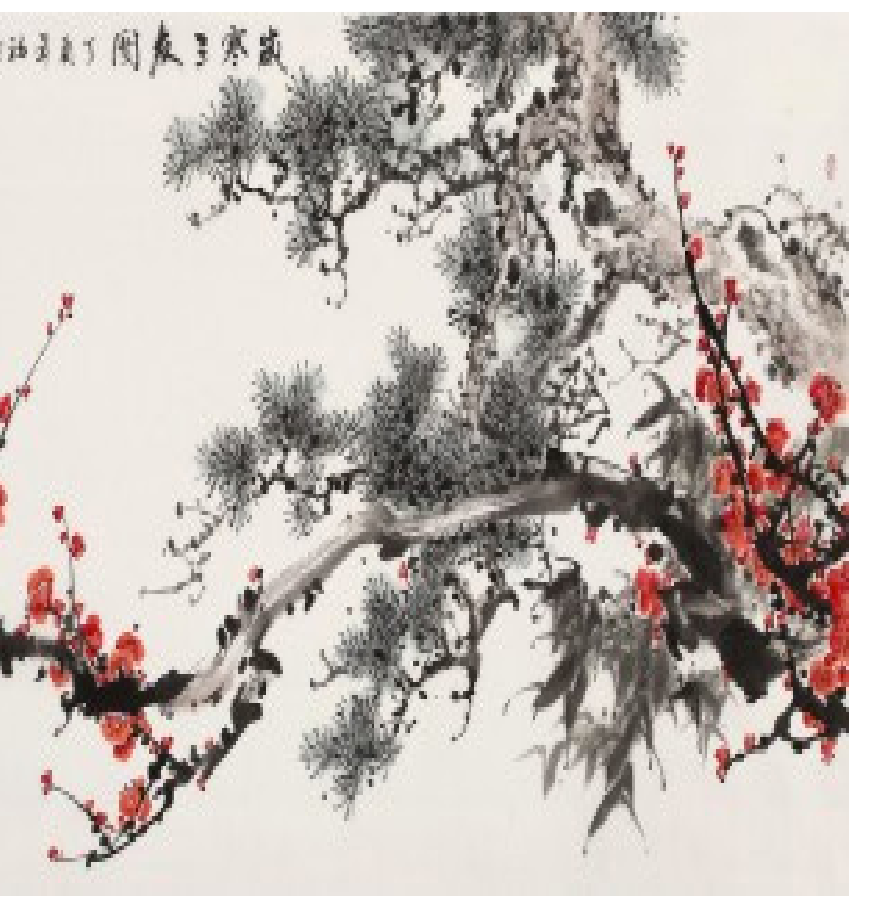

figure 47. "Three friends in the winter", pine, bamboo and plum. Bencai $Z$

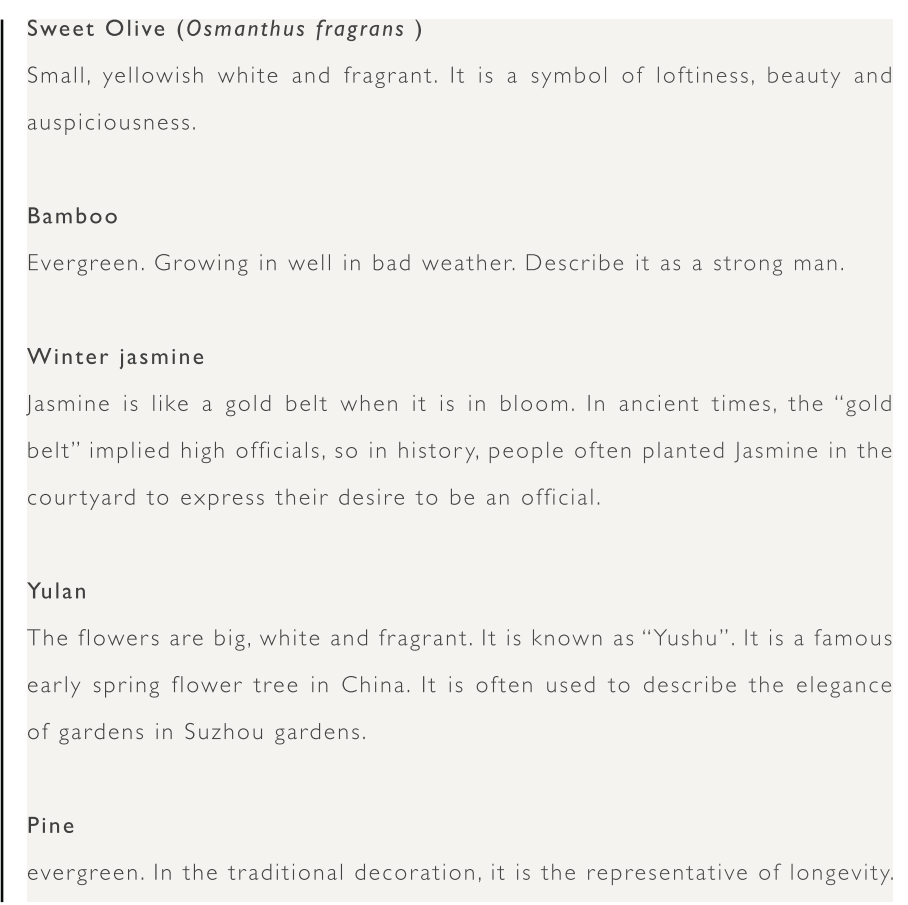

figure 48. examples of narrative plants in Chinese culture

Overall, in the development of Chinese traditional gardens, Confucianism and Taoism are intricately intertwined. They work together on both a physical and mental capacity to develop a Chinese landscape with cultural thoughts. As this design research is based in China, this design research will ultilise the principles set upon by Chinese philosophy, as a way to emphasise and acknowledge Chinese culture 


\section{LANDSCAPE NARRATIVES}

The narrative in landscape intercepts with site's accumulated layers of history and can be changed over time (Potteiger \& Purinton, 1998). Potteiger \& Purinton (1998) defined landscape narratives linking what is treated as a material or visual scene with the less tangible, but no less real, network of narratives" (P7). The methods to make a landscape narrative are mentioned in the book and can be summarised as follows:

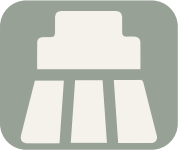

- Naming (naming something to make people feel interesting about the space),

- Revealing (creating suspense of the space to affect the emotion of the users, invite users to mask and unmasking the suspense about the place)

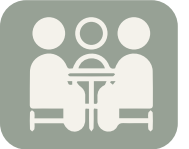

- Gathering( miniature landscapes condense events into a coherent and clear story)

- Opening( draw distinctive boundary between users) (Potteiger \& Purinton, 1998).

Furthermore, to design based on narratives, Landscape Architects can utilise natural materials, such as trees, rocks, ground or weather, to serve as symbols (Potteiger \& Purinton, 1998). 
Aesthetically pleasing environments provide valuable experiences to users that can improve human quality of life (Chenoweth \& Gobster (2019). Jones (2013) discussed Landscape as a place that showcases human aesthetic and illustrates human culture and history. Jones(2013) emphasises designing a space to be unique with its own identity, highlighting the local landscape's extraordinary ecosystem. Koolhaas et al. (2013) discusses how they designed for uniqueness in Berlin, which was achieved by choosing areas that already conduct a robust existing identity. By highlighting what is important and meaningful about

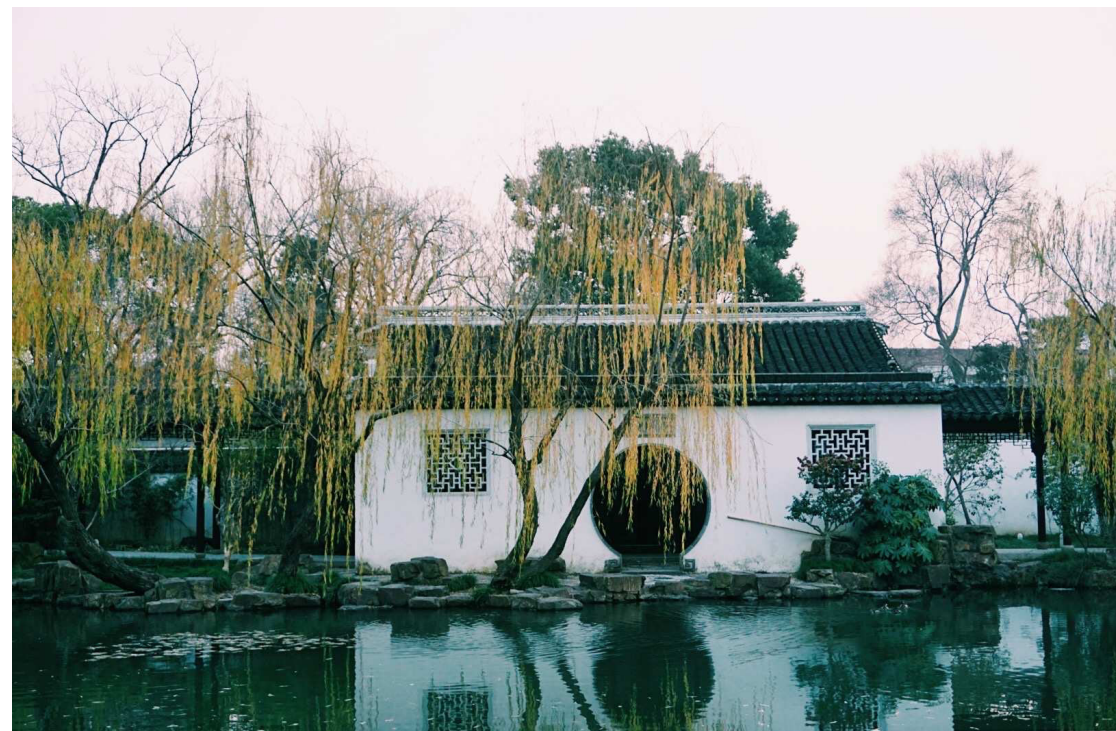

figure 49. Shallow hidden by using the wall, Suzhou the site, it allows for perseveration of critical elements. This has led Berlin to being more legible and celebrated by foreigners and in particular, by locals (Potteiger \& Purinton, 1998). To conclude, landscape narratives has brought stories and site history to users, by featuring the identity of different space' (Potteiger \& Purinton, 1998).

Landscape Architects all over the world have experimented with how to make a Landscape narrative. They each offer different methods on how to develop a Landscape and encourage special human experiences through the act of telling stories.

In China, the use of vegetation and abstract shaped rocks showcase the personality of the privately owned garden. Also, to tell these stories, designers prefer to use methods like penetration, Hidden and Reveal, Shallow Hidden, Shallow reveal, and suddenly change a scene to increase the user's impression of Lou (20II). 
In Europe, designers thought to make a landscape narrative by dividing the location into several smaller sites with connected the scene with story-telling (Calder, 2006). The landscape is working four dimensions of time, as the users move through the garden on a journey from one place to another (Calder, 2006). A practical principle used within Europe Landscape is working with sculpting and manipulating the vertical space as a horizontal plane (Calder, 2006). It is similar to the narrative Landscape in traditional Chinese gardens feature, where the garden evolves through movement. Besides, users cannot see the entire garden from any location in the garden. Users can collect stories in each stopping point throughout the journey, abstractly, it can be considered like chapters in a novel.
Overall, landscape narrative can always, if done correctly, highlight the identity of a site. The method to make a landscape narrative from different literature pieces can be the possible way to use it in the design in Lake Fuxian.

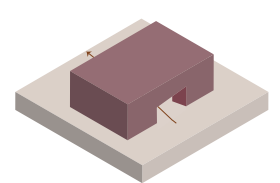

penetration

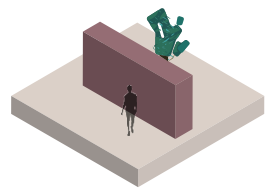

hidden

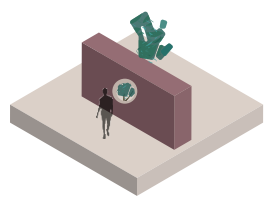

shallow hidden

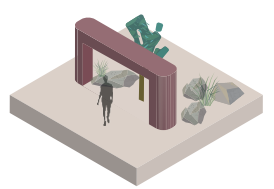

reveal

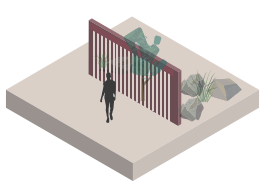

shallow reveal

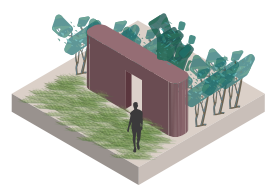

suddenly change a sene

figure 50. Methods for landscape narrative in Chinese traditional garden 
PRECEDENT REVIEW

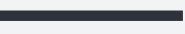




\section{STRUCTURE}

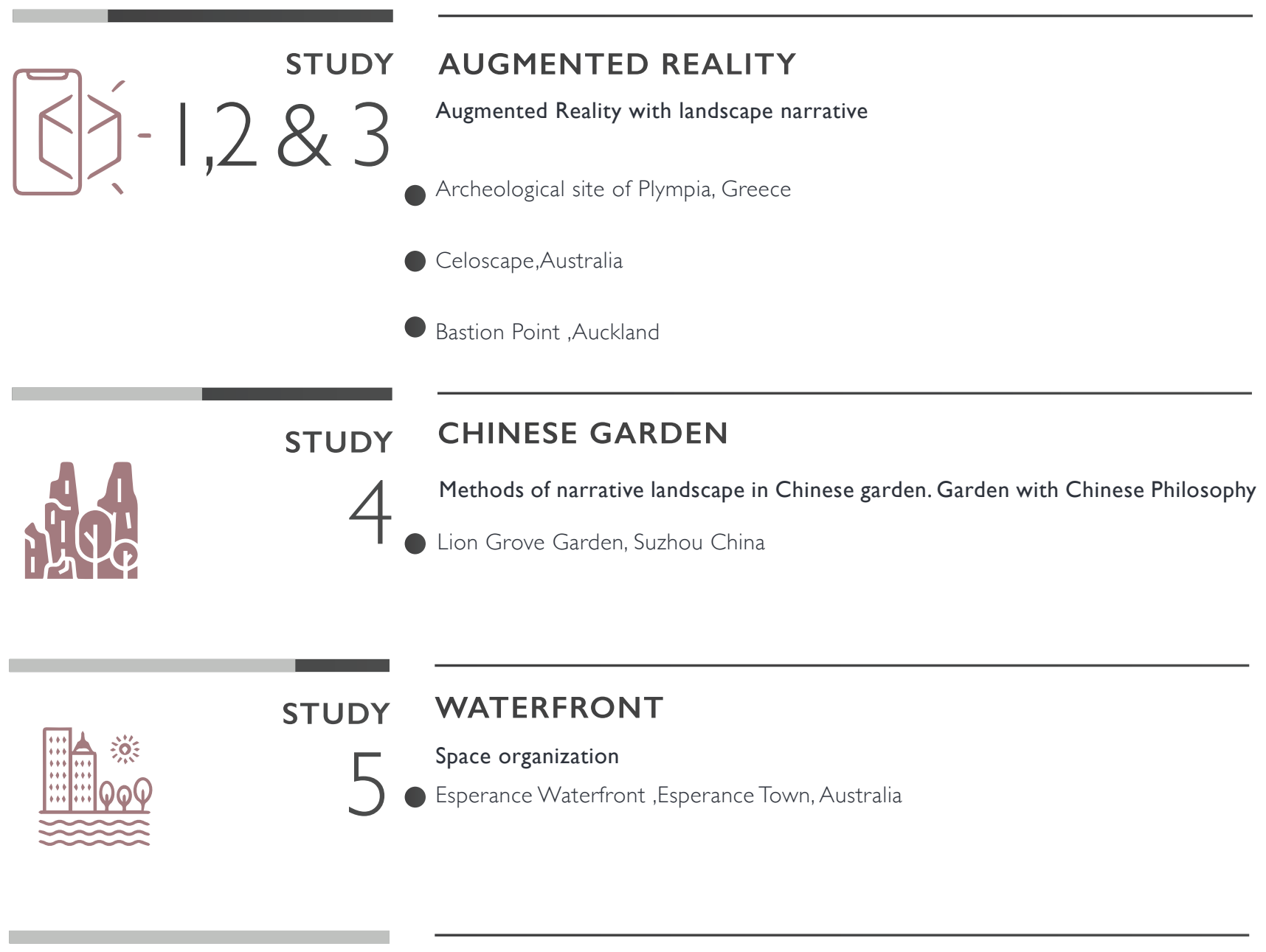




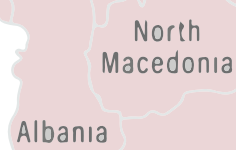

Greece

\section{O ARCHEOGUIDE

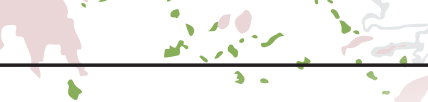

ARCHEOGUIDE is an IST (Information Systems \& Technology) project funded by the European Union. It aims to provide a cultural tour to visitors by personal devices in the archeological site of Olympia in Greece (Vlahakis et al, 200I). The design research was in 2000, where mobile phones were not yet popular, however the designer allows people to utilise a computer and witness the hidden space of the site. The 3D reconstruction of monuments and artifacts are presented to the user through a particular AR interface with natural surroundings and audio commentary (Vlahakis et al, 200I). It rebuilds how the architecture used to be in conjunction with what is present, through AR.
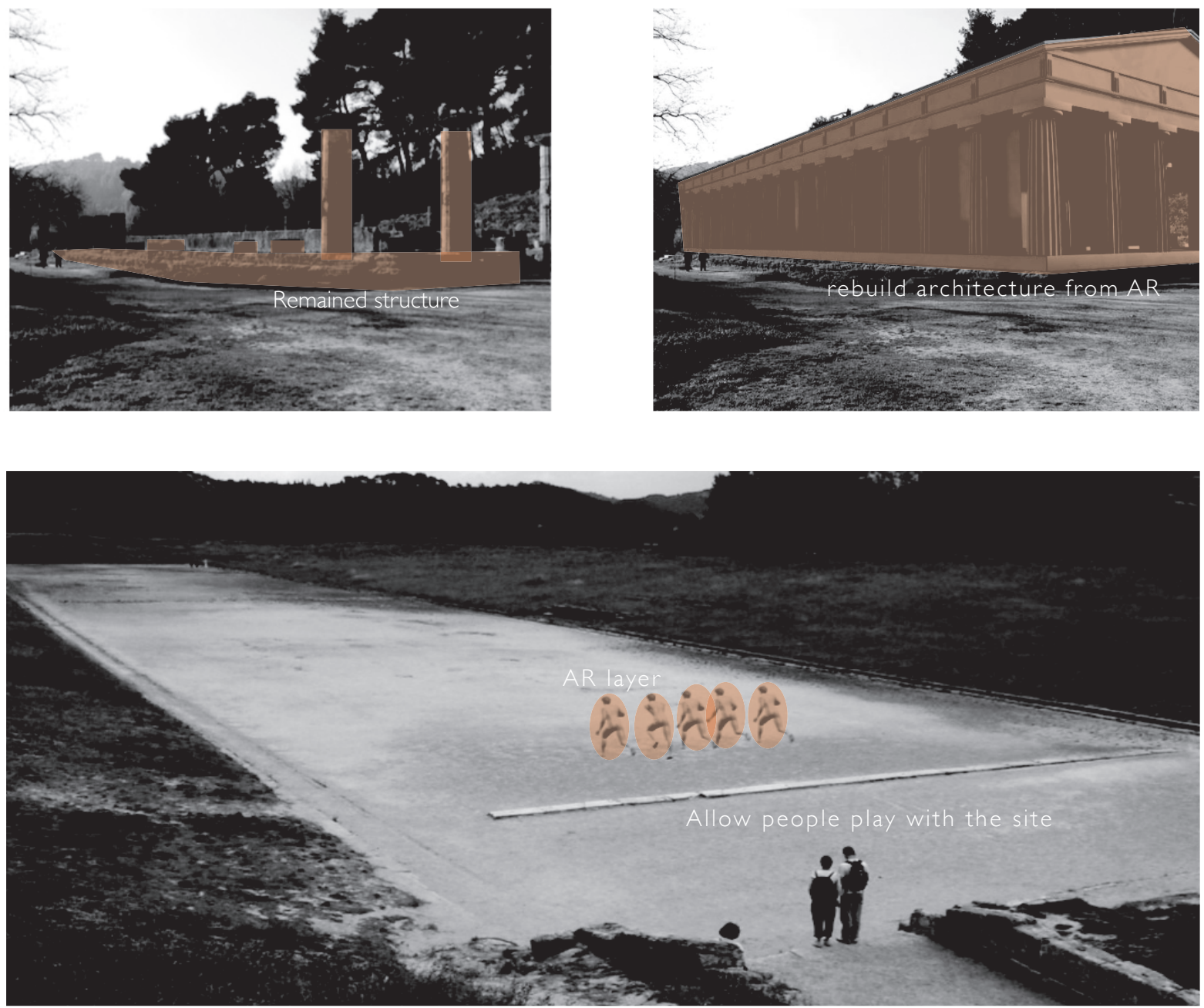
Additionally, the designer included accurate virtual human models within the software. They were historically exact and included mannerisms to the era they were depicted in (Vlahakis et al, 200l). However, the attention to detail that is needed, results in a high cost of capturing data, making it not ideal for wide commercial use.

Due to minimal change in environmental conditions throughout time, there was little influence within the AR of the landform and natural conditions, only human and built forms. However, this provided visitors with an educational journey via their phone, by adding layers to existing heritage. It is an instructive and effective way to show the roots that informed the future.

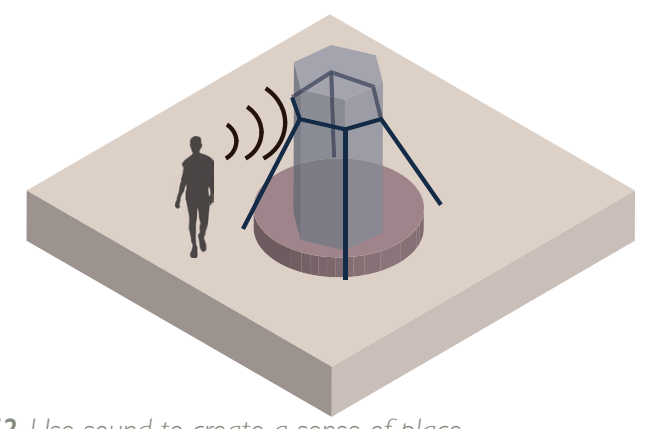

figure 52 . Use sound to create a sense of place

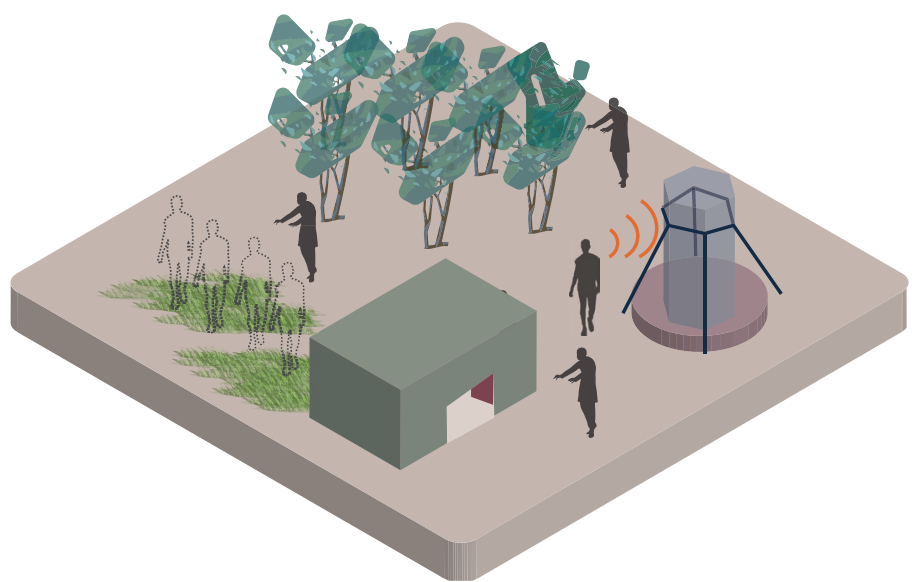

figure 53. Location tracking in different area
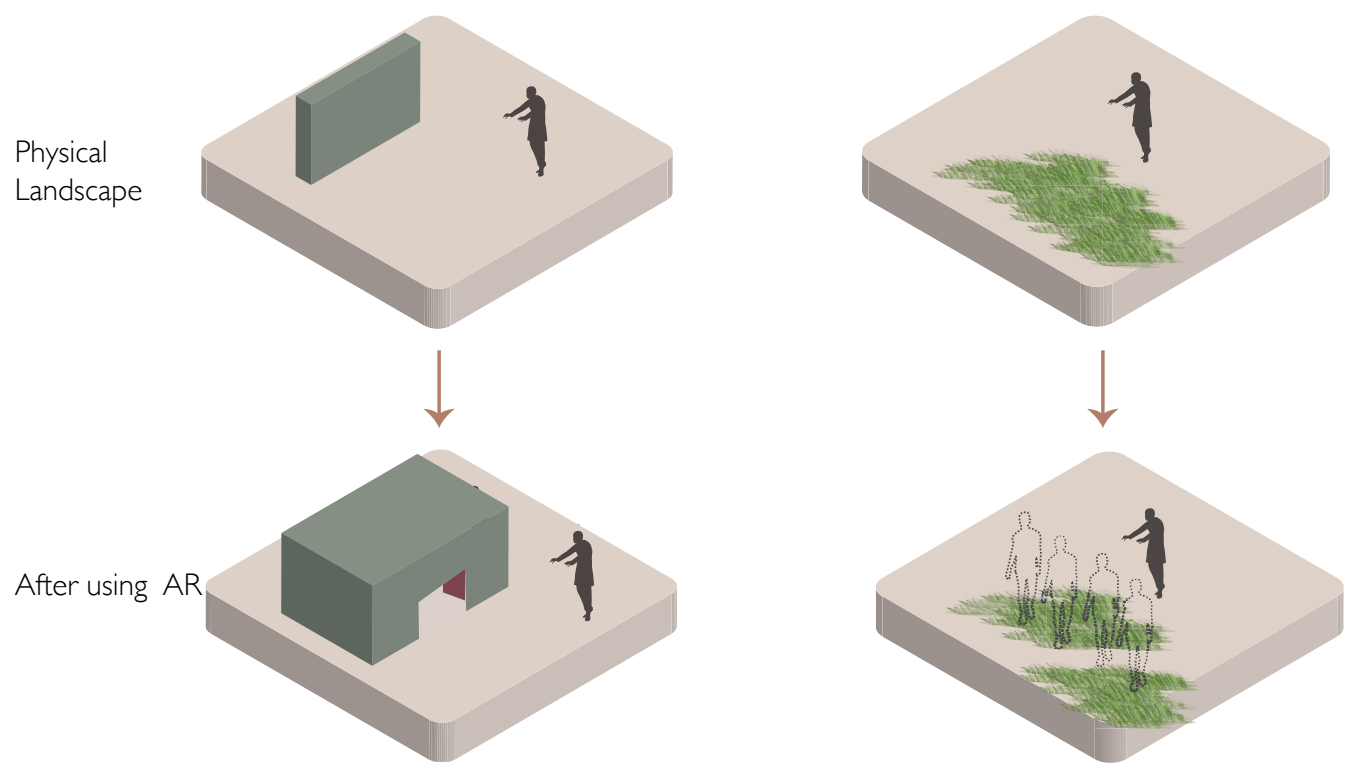

figure 54. Design of Archeoguide, Physical site and AR layer 


\section{CELOSCAPE}
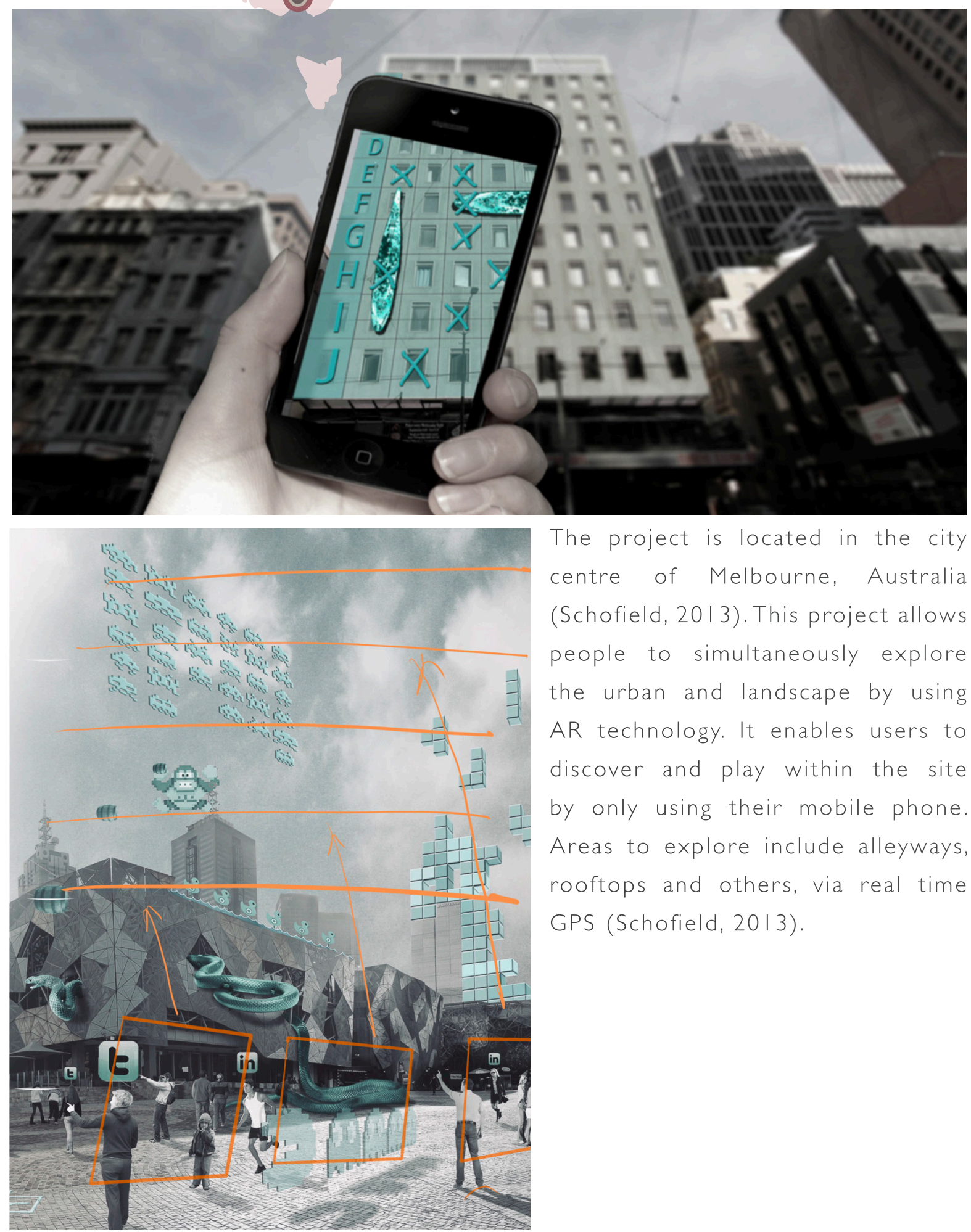

The project is located in the city centre of Melbourne, Australia (Schofield, 20|3). This project allows people to simultaneously explore the urban and landscape by using AR technology. It enables users to discover and play within the site by only using their mobile phone. Areas to explore include alleyways, rooftops and others, via real time GPS (Schofield, 20|3).

figure 56. location tracking, Celoscape 
The functions within the app include(figure 57):

- Identity :identity the space that has things to explore.

- Create :people are allowed to create a story like a draw the things they interesting, Play: allow play games on the site by using their applications.

- Strategy :tell the stories of the space and exercise users' focus and mind, it would help people have a stronger impression for the place.

- Social :meet new people and make friends which is functional. Due to the design, people are more welcomed to go to urban space and stay longer.
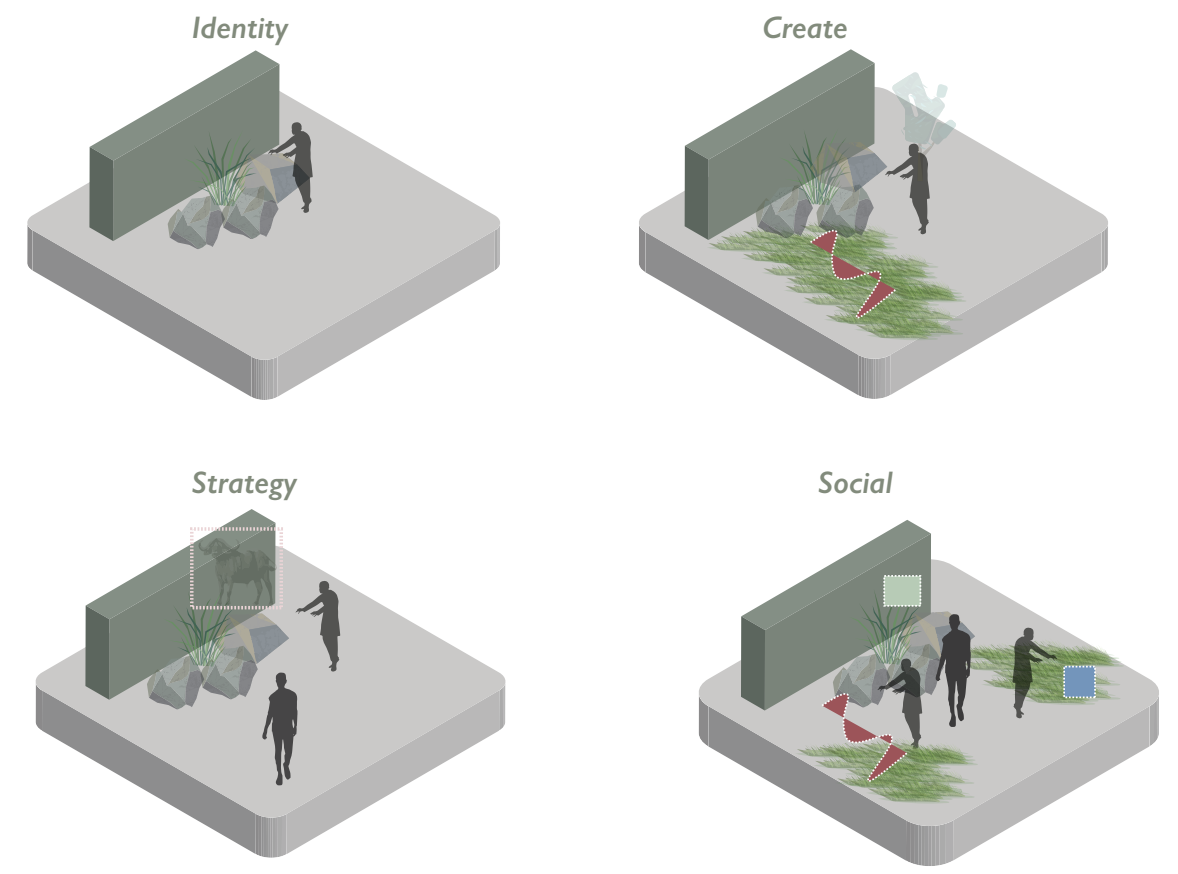

\section{Weaknesses}

The design is based within a crowded city and as a result, might affect the safety of pedestrians or and the app users. Focus on mobile devices minimises social awareness as there is no specific stopping locations to utilise and view the digital information(figure $58)$.
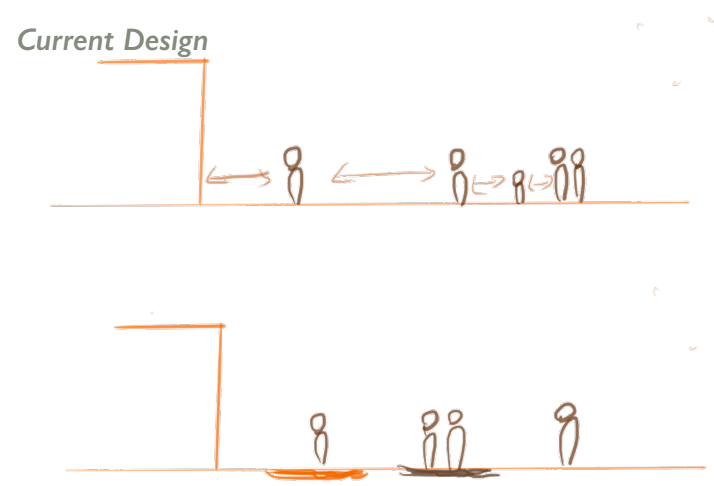

Potential Development

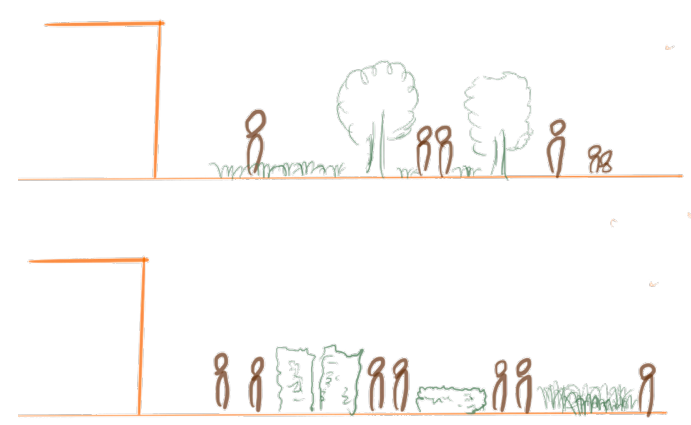

figure $\mathbf{5 8}$. safety of the users 


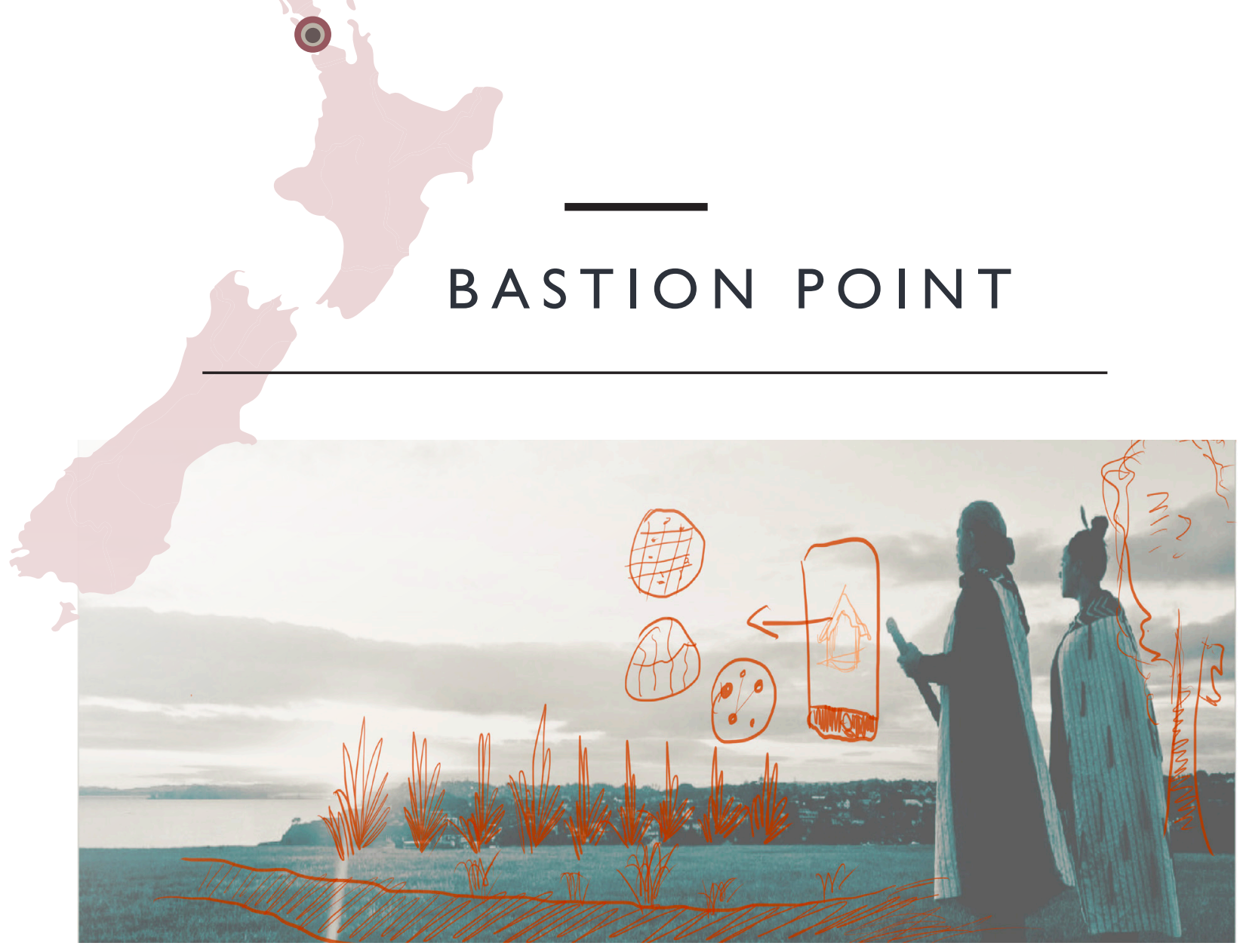

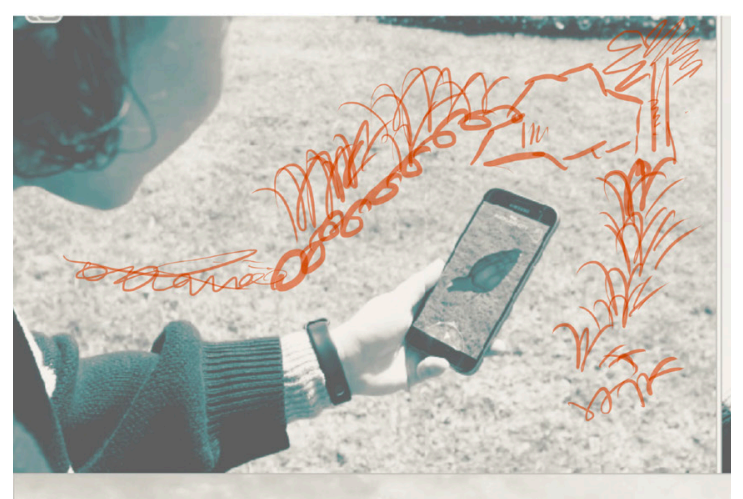

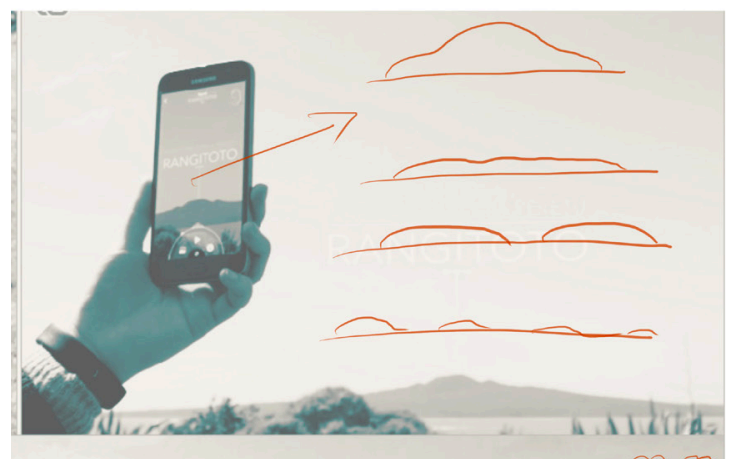

figure 60. Potential development for the design
This is a mobile app that allows users to have a walking tour around Bastion point in Auckland (Williams, 2018). It is produced by Virtual and Augmented Reality Studio and Ngā Whātua Ōrākei. Unlike the Celoscape project, it uses geo-tracking technology to identify specific areas to educate people on the journey of Māori culture (Williams, 2018).It highlights sites that have specific historical and cultural values by adding layers to the physical landscape on a Smartphone application (Williams, 2018). When people visit a site that has been registered within the app, simply pointing the camera at the geological 
landform will overlay its history in an engaging formation (Williams, 2018).

A drawback for this application is that there are often not many changes from the physical landscape to the digital one. This limits the engagement of the app and visual understanding of the space(figure 60). However, for areas that are heavily documented within the app, the information is invaluable. People not utilising the app cannot fully appreciate the importance of the site as they do not realise its past.

The use of $A R$ on the site makes the location playful and interactive. After the journey through the site with the guidance of the application, users will gain knowledge about how the site has become what it is known as today. However, the design for the physical landscape did not get enough development, which might decrease the interest in wanting to know the space.
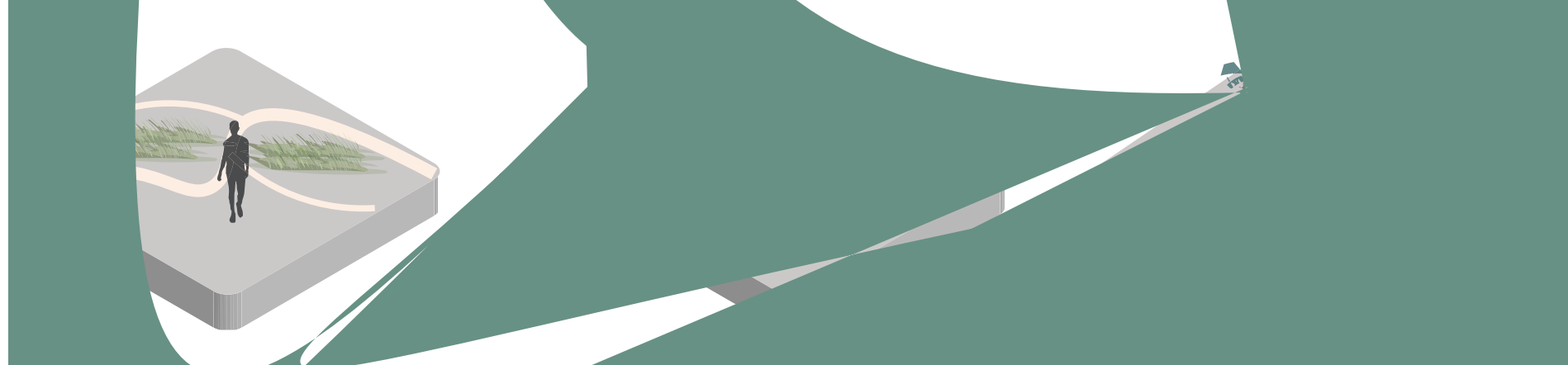

figure 6I. key ideas of the precedent 


\section{Lion Grove Garden O}

Lion Grove Garden is a heritage garden in Suzhou, China. It has more than 700 years of history (Wenku, 2013). The total garden covers an area of about 10,000 square meters and the central pond occupies a quarter of the total area of the park(Wenku, 2013). Comparing to other traditional Chinese gardens in Suzhou, this garden is at a much smaller scale. However, it does include many interesting elements such as an artificial labyrinth with many levels, caves, and peaks.

The most famous spot within the garden is the maze and cave, made by groupings of Taihu Stone (a type of natural stone in China). The unique shape of the rocks has a similar appearance to lions, many people come to the site to count how many lions are actually in the garden. Therefore the park called Lion Grove Garden(Wenku, 2013). Furthermore, the most memorable feature of the garden is the amusement and excitement of traversing through the maze.

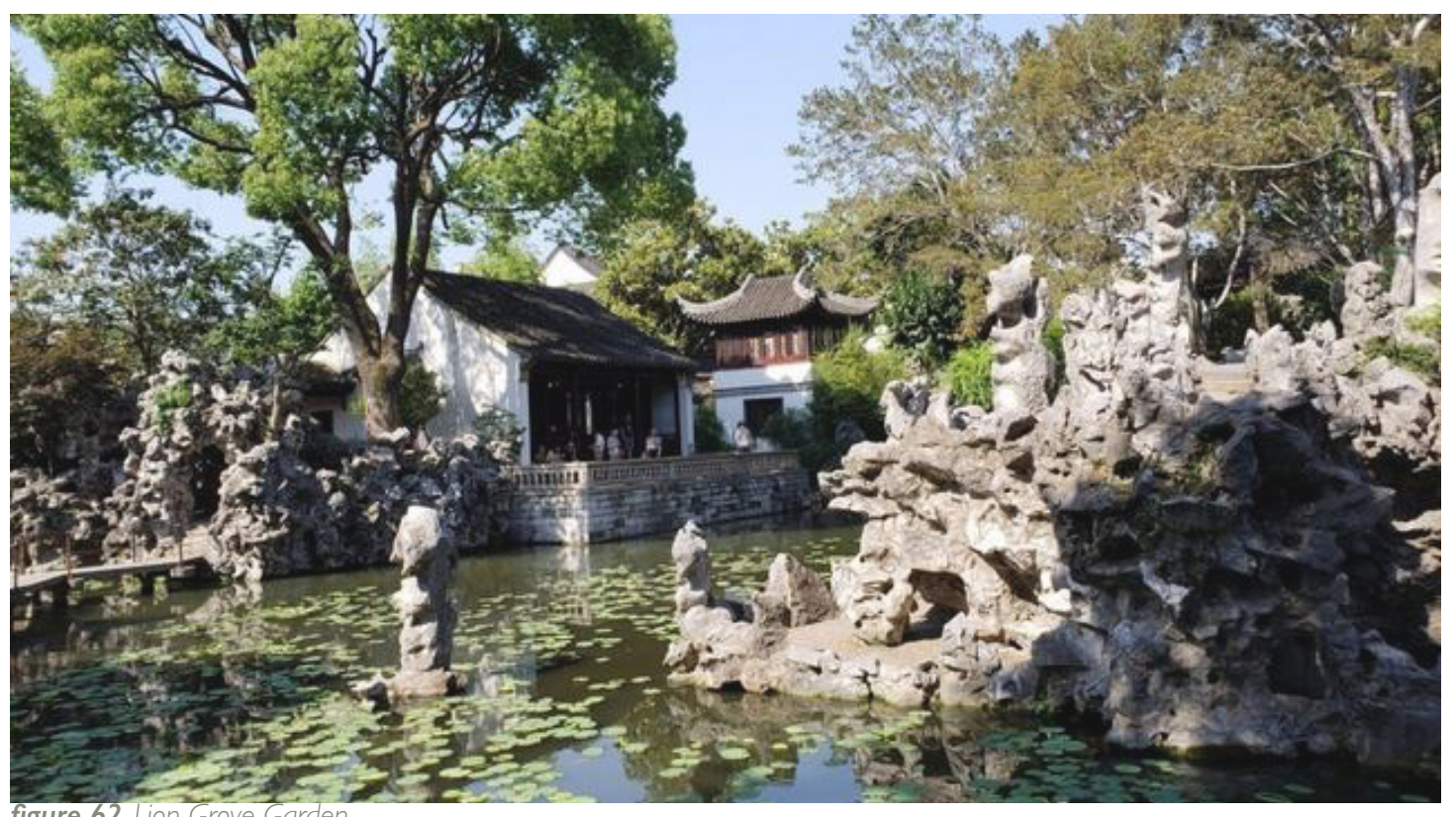

figure 62. Lion Grove Garden 


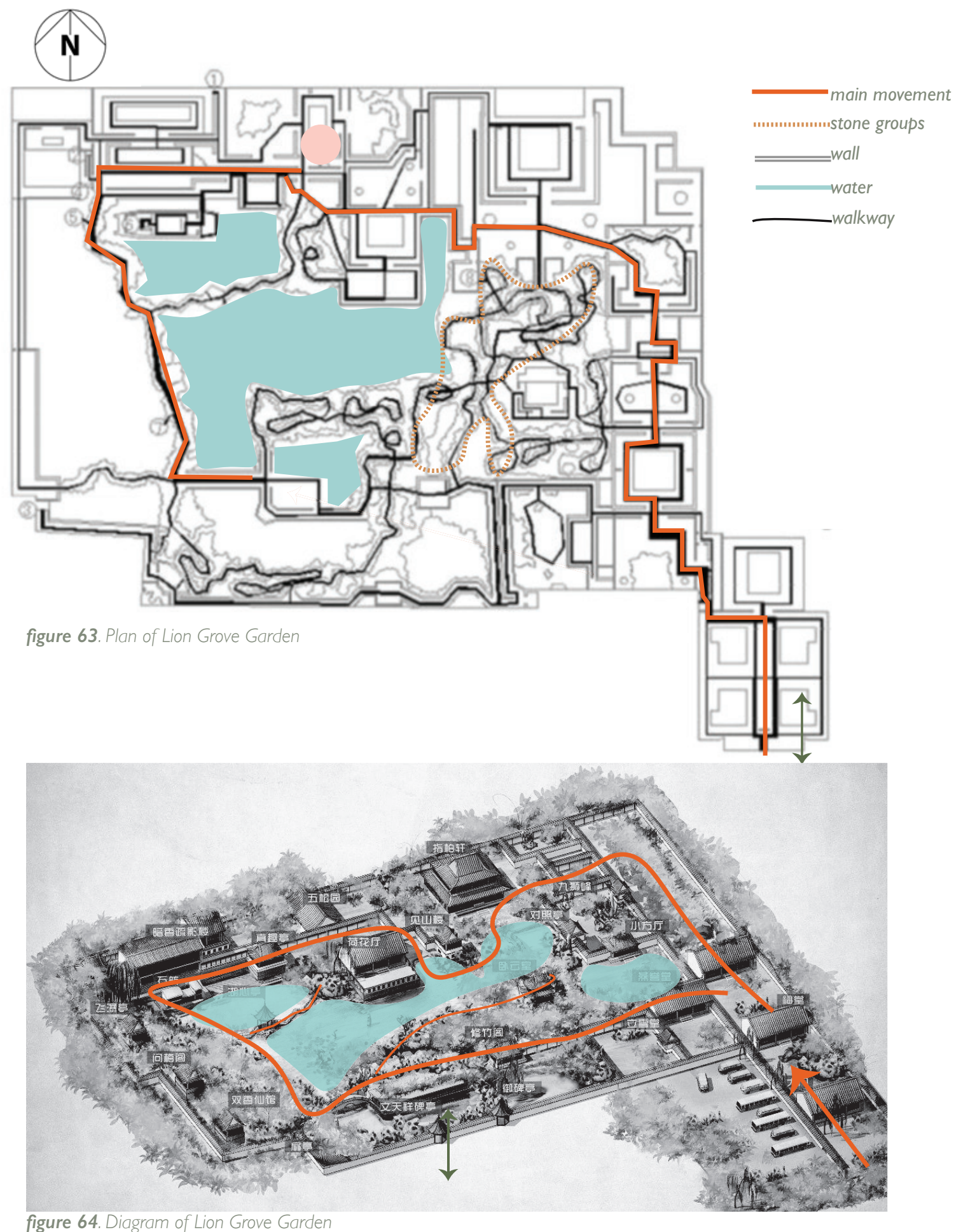


When people are walking in the garden, they do not realize that they have walked into a maze. The interesting element is that people can see through the maze, but it does not necessarily mean that they can walk through it(Wenku , 2013). The time it takes to traverse through the maze depends on skill and luck, varying from five minutes to over an hour. The garden includes the method used with penetration, hidden and dew, shallow hidden, shallow dew and suddenly change a scene. (Wenku, 2013). It also collects the view from their windows(figure 66). Every view of every window in the garden is like a Shanshui Painting.
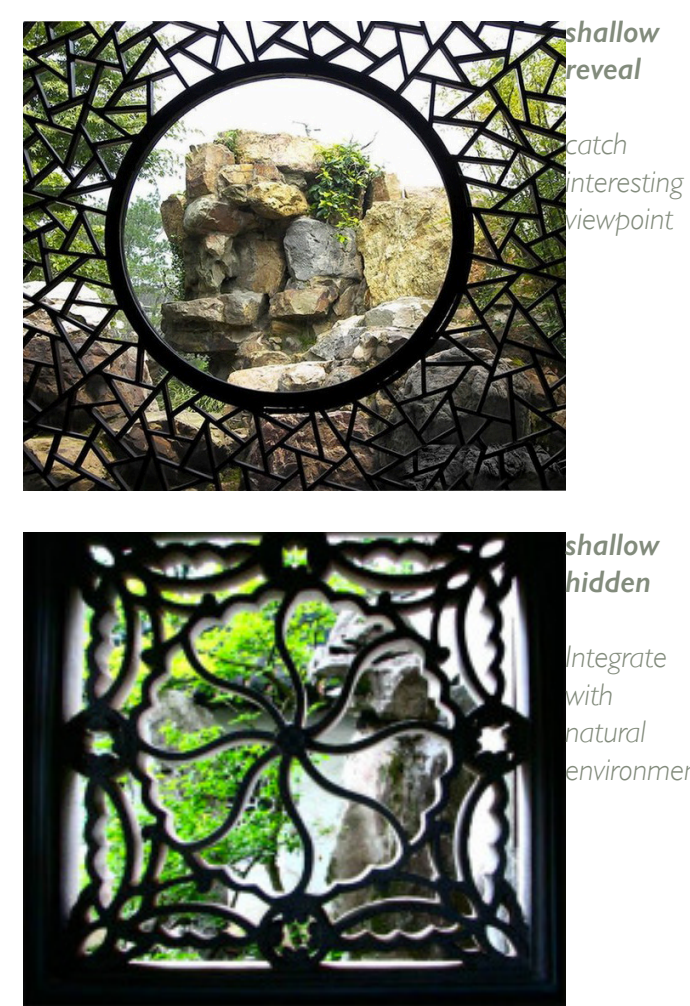

figure 65. Windows of the garden
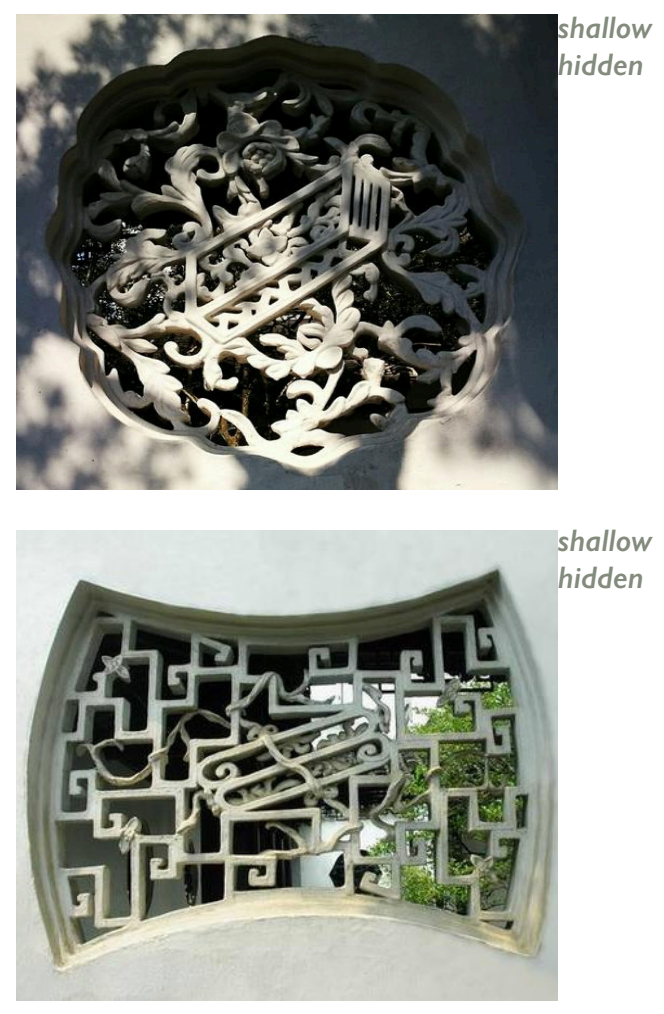


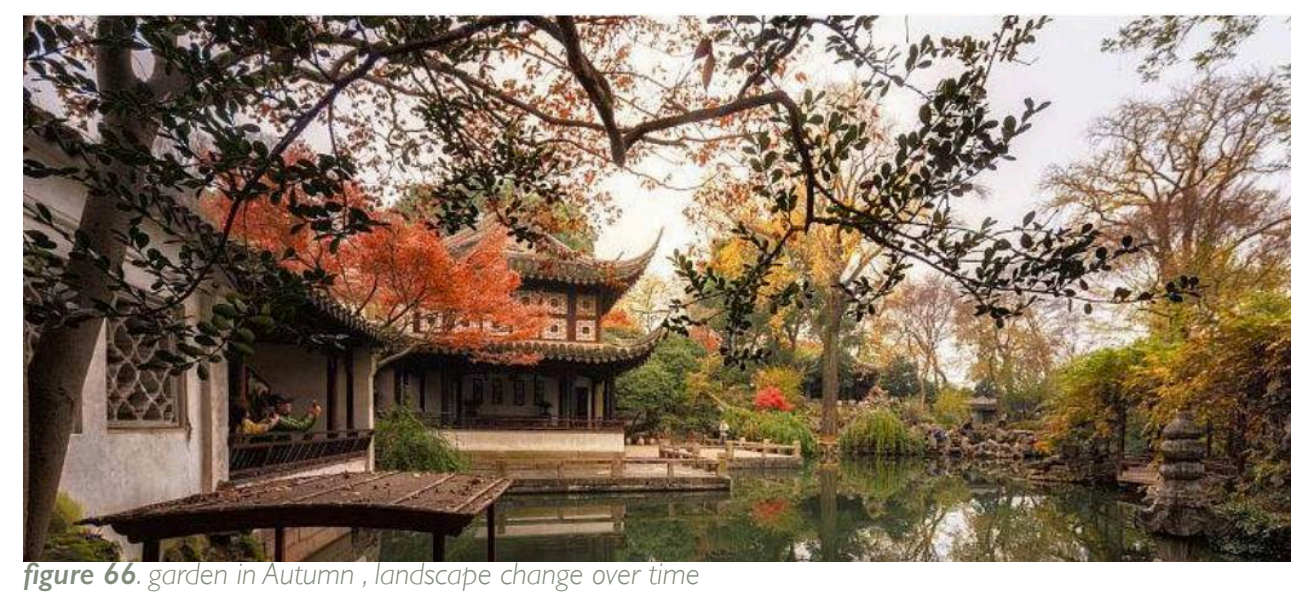

Overall, the design of the grove garden used many concepts of Chinese philosophy. This includes the material used and the elements in the garden include Wuxing (Metal, Wood, Water, Fire,Earth). The techniques from traditional Chinese culture creat multiple journeys that are never the same, resulting in a unique experience for every visit. Also, it has a sense of traditional Chinese identity, which is very easy to differentiate from a foreign culture. The ways the designer created different spatial qualities, to subtly inform the visitors the narrative of the space, would be a potential to use within design iterations.

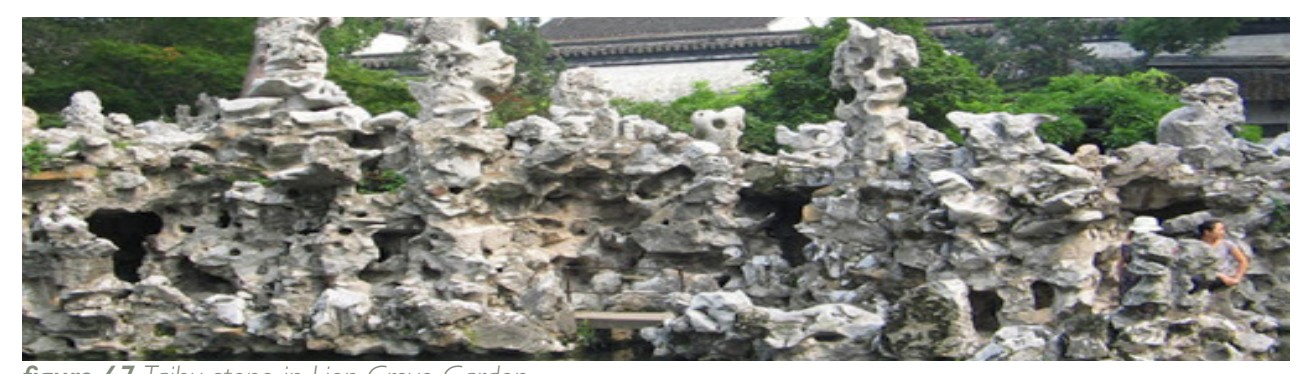

figure 67. Taihu stone in Lion Grove Garden. 

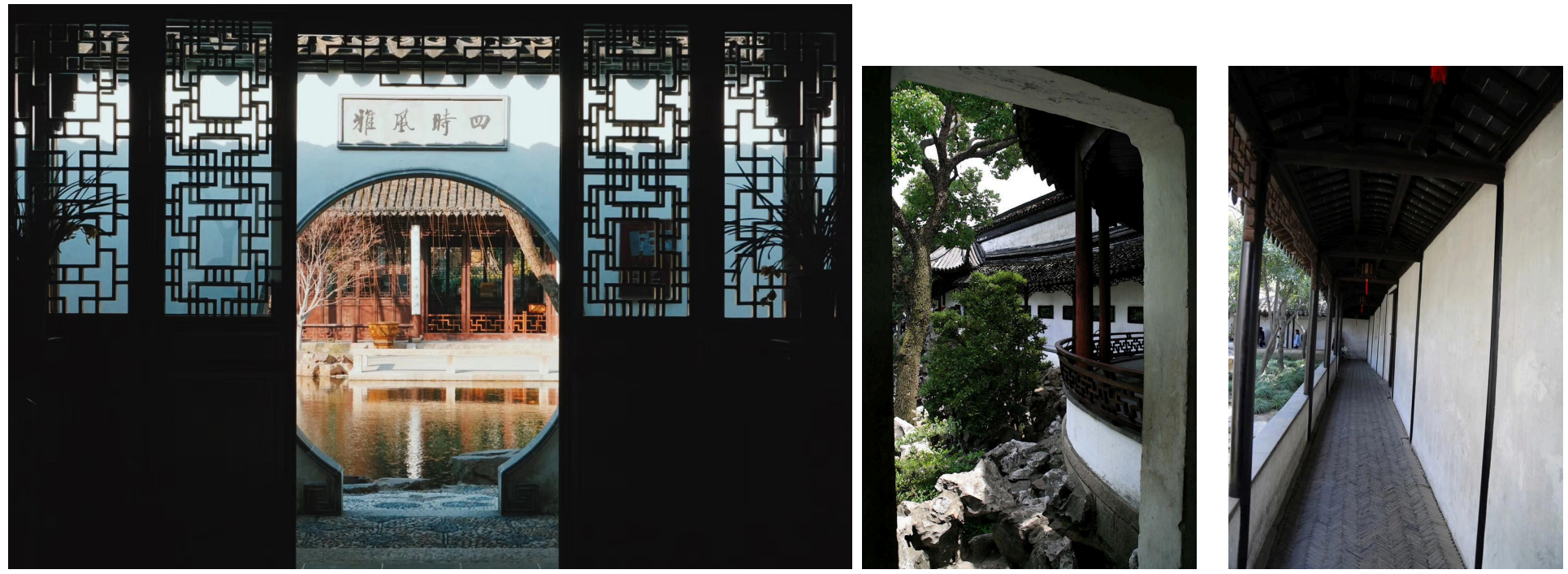

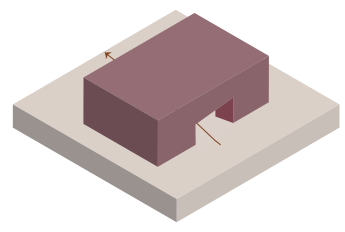

penetration

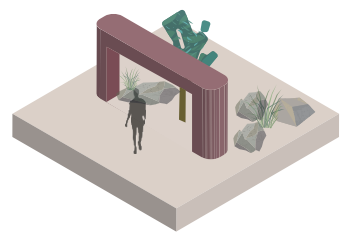

Reveal

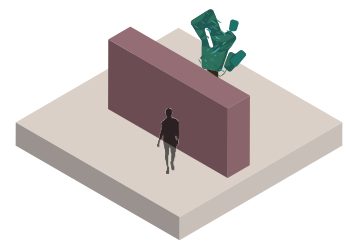

hidden

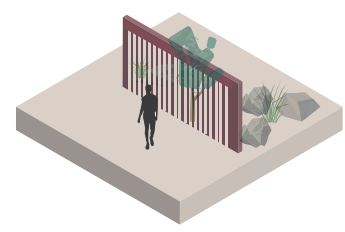

shallow reveal

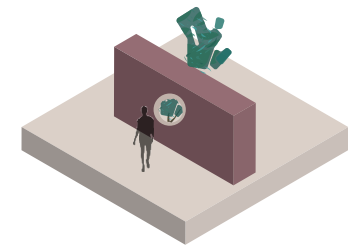

shallow hidden

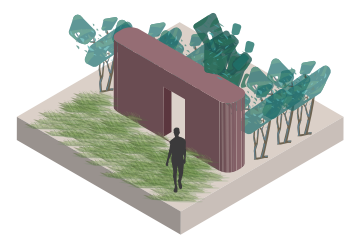

suddenly change a sene

figure 69. methods used in Lion Grove Garden 


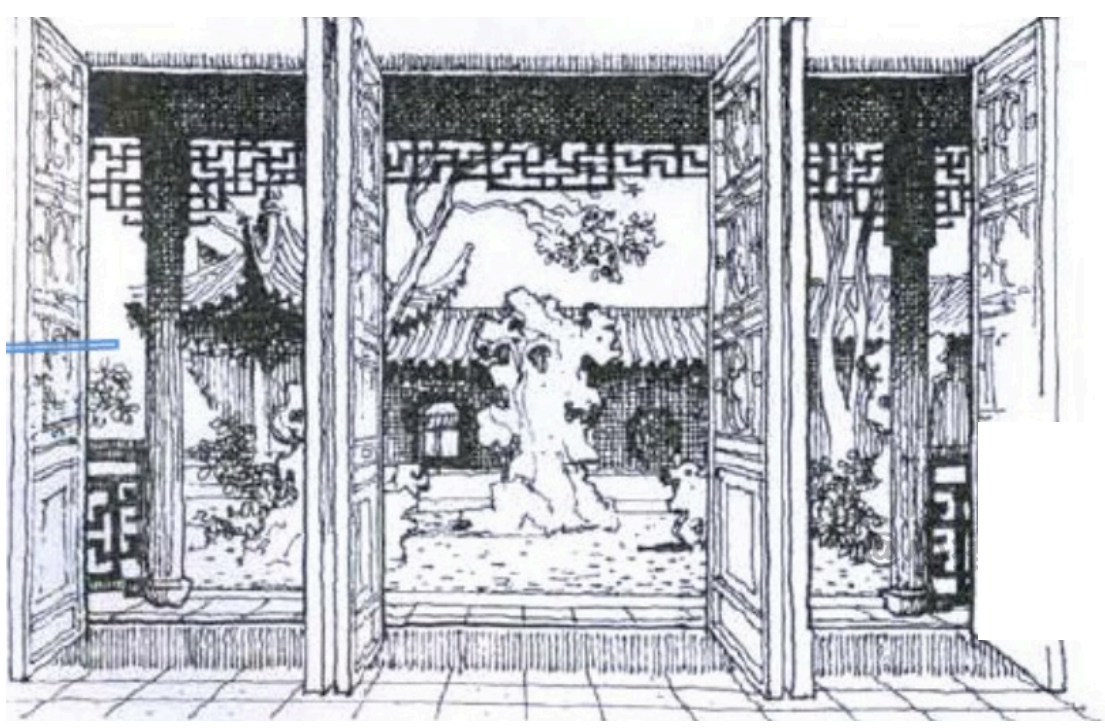

figure 69. Reveal method

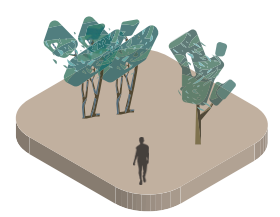

contrast between sparse and dense

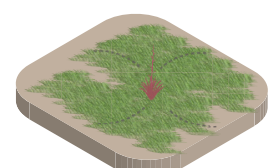

contrast between dynamic and static

the layout of landscape elements require appropriate density and create a contrast.

eg:if there are only three trees planted, two trees should be close to each other, and the third tree should be far away

can use colour to make a contrast.

for example, in Lion grove garden, a grassland suddenly reveal a red flower,.designer in China thought it can make the landscape dynamic

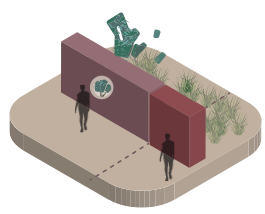

slowly show the landscape to visitors, and make a contrast between hidden and reveal

Contrast between hidden and reveal

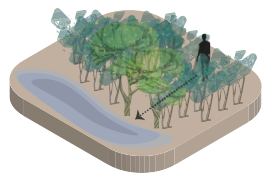

similar with method, that suddenly change a scene 


\section{Esperance Waterfront}

0

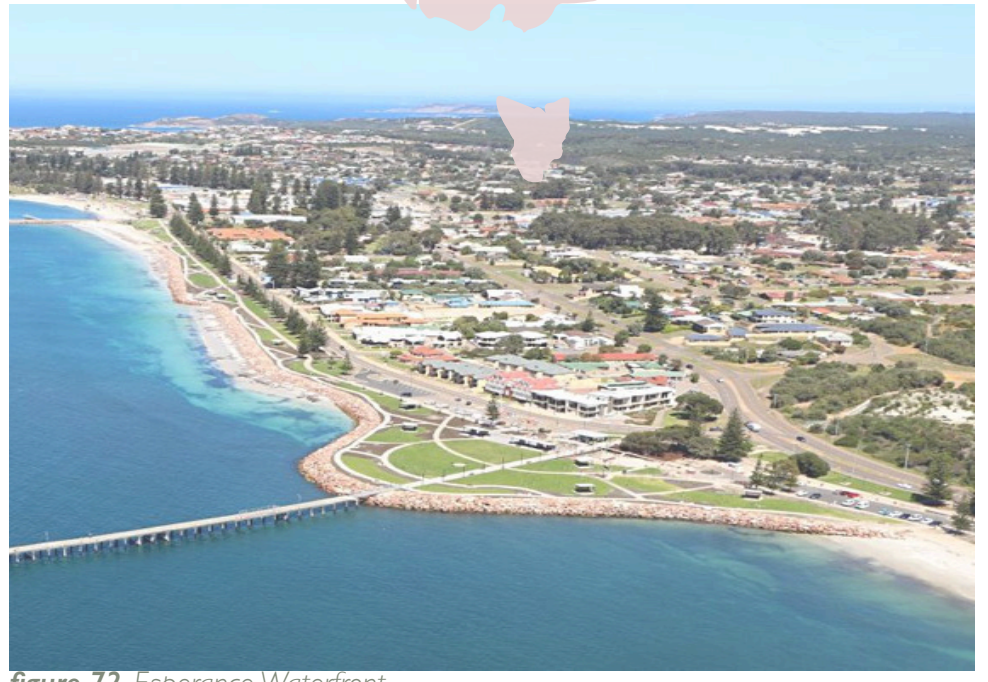

figure 72. Esperance Waterfront

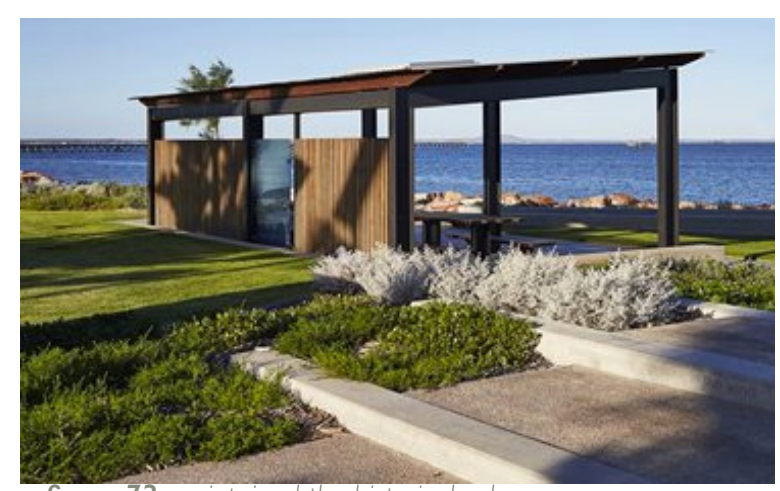

figure 73. maintained the historical values

The design of the waterfront aims to tell the story of Indigenous people, natural land formations and early settler history $(\mathrm{Ou}, \mathrm{n} . \mathrm{d}$.). To reinforce the sense of place, the designer used local materials in the form source from reclaimed timber, and railway lines, which was converted into a serviced jetty, benches and walkways (Ou, n.d.).(figure 73) This design as a new attraction and offers both local, family and tourists come and use the site. Food trucks and pop-up events, can be held on the vast lawn space in waterfront $(\mathrm{Ou}, \mathrm{n} . \mathrm{d}$.). The designers used interpretative signage, shelters, and public art to highlight areas of interest, such as the foreshore jetties and the unique flora and fauna.

As a successful waterfront design, it differentiates a large space into multiple smaller spaces (Ou, n.d.). People have the option to navigate freely through the site, choosing to either travel quickly or take the longer scenic route. It is a space for community, bringing together multiple different demographics through various design iterations (Ou, n.d.). 
Journey and differentiate space

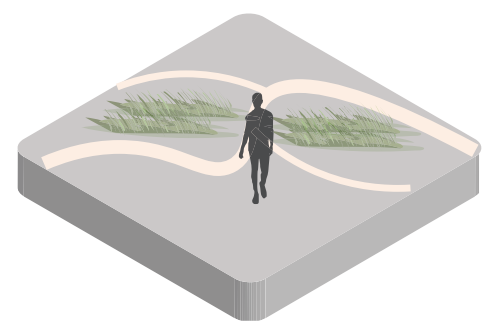

Maitain the historical things

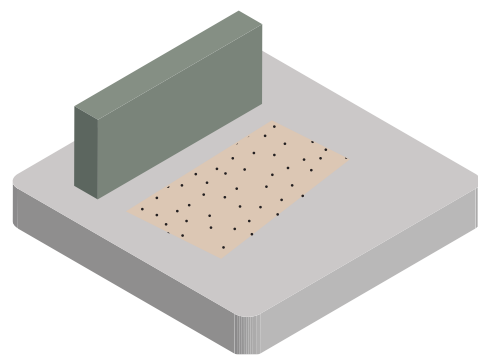

figure74. method used in the design

Overall, the techniques create a multifunctional space, perfect for all activities and people.

The way the design manages spaces via the allocation of function for these areas, can be replicated through future designs. Additionally, the way the designers managed confusion from the multiple pathways with signage should be replicated within the final design.

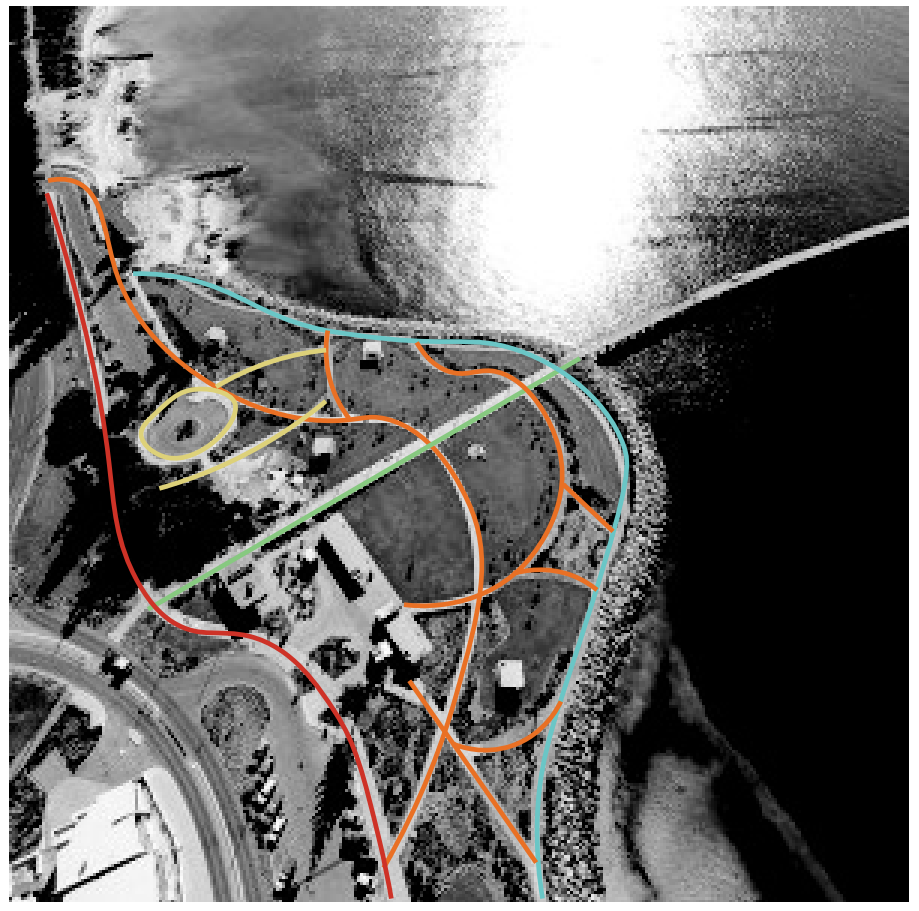

figure75. different journeys on waterfront

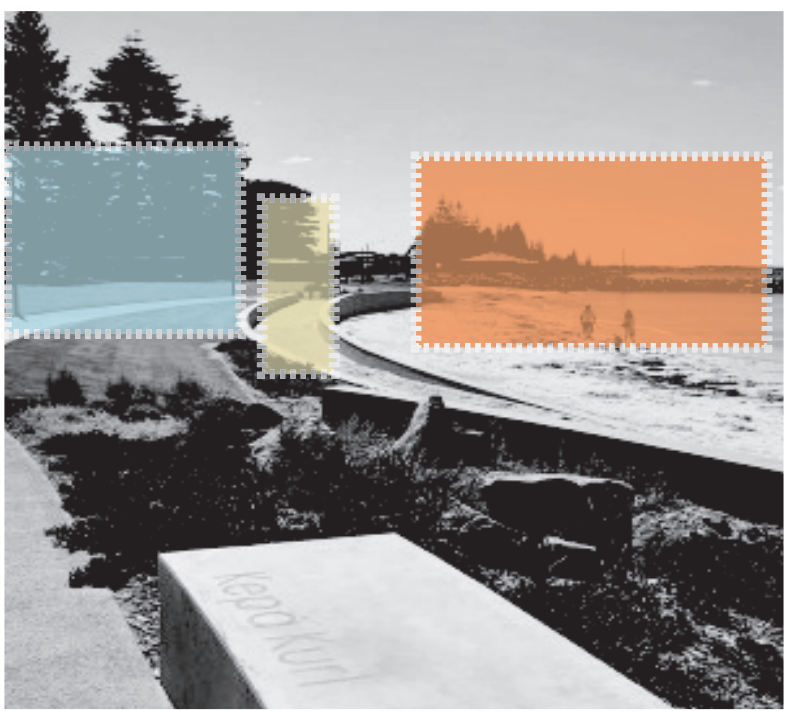

figure76. space organization

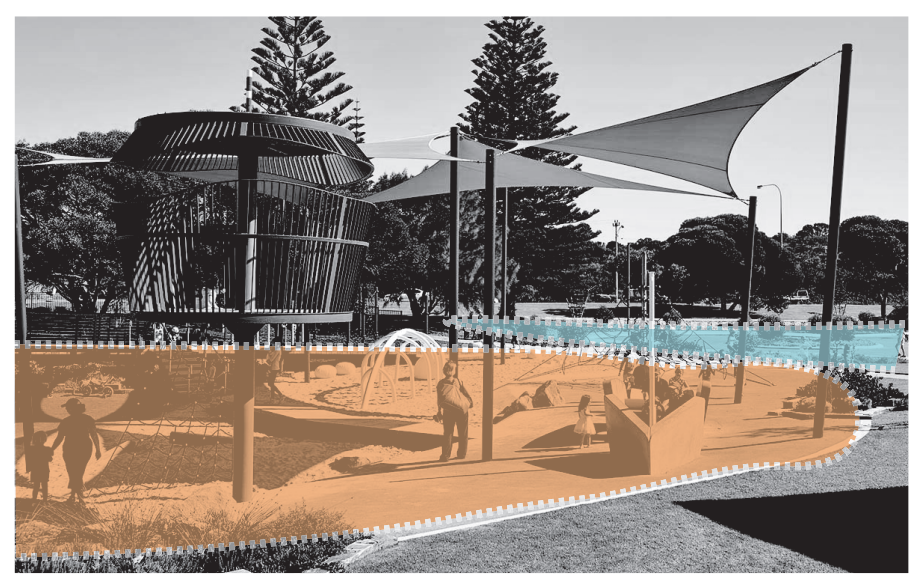

figure77. transition between walkway and children entertainment area 


\section{DESIGN STRATEGIES SUMMARY FRO}

Experiences/ "Wushi" in Confucianism

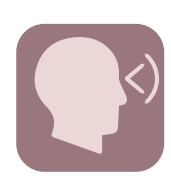

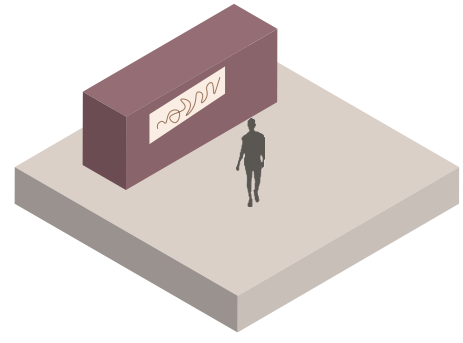

sight

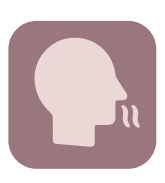

smell

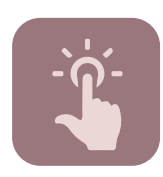

touch

Narrative strategies in China

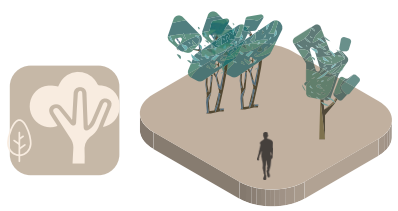

contrast between sparse and dense

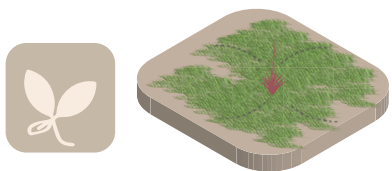

contrast between dynamic and static

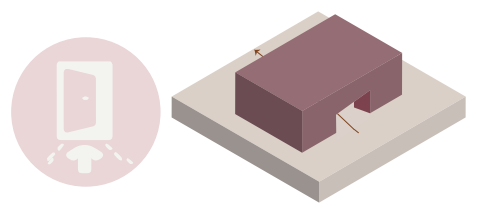

penetration
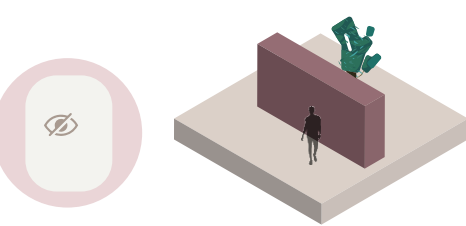

hidden

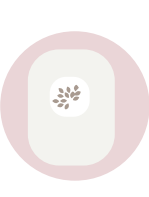

shallow hidden
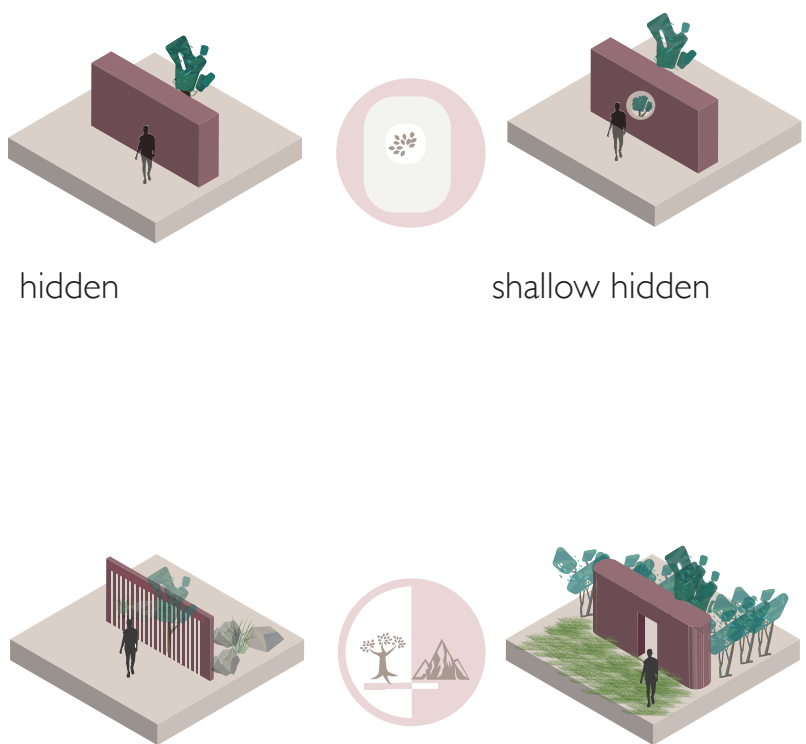

shallow reveal
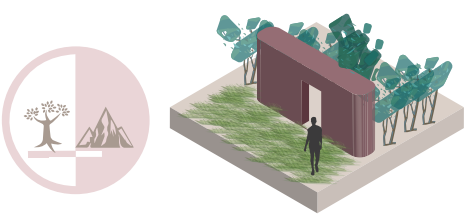

suddenly change a sene 


\section{LITERATURE AND PRECEDENTS}

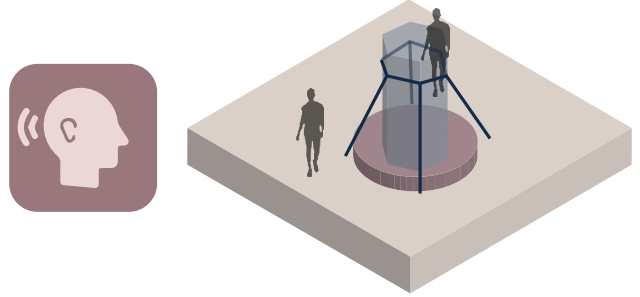

sound

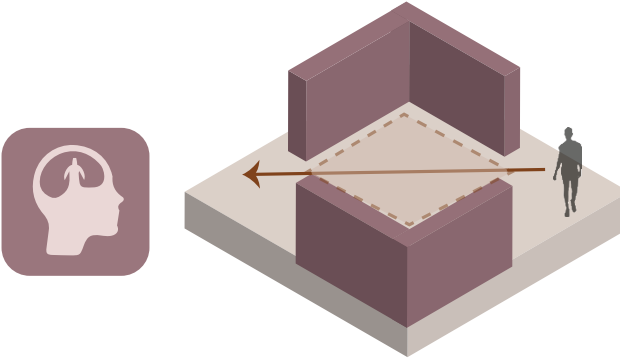

preception

Narrative with Augmented Reality

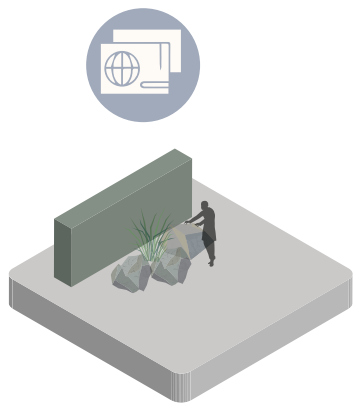

Identity

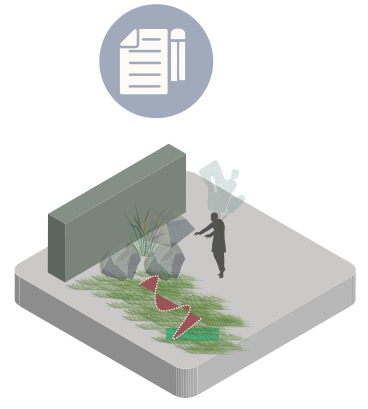

Create

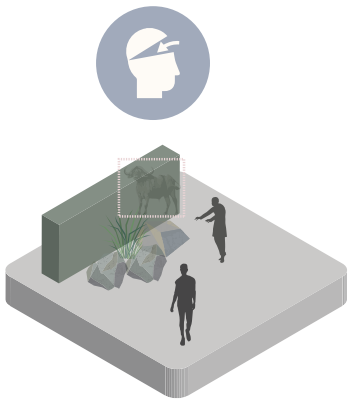

Impression

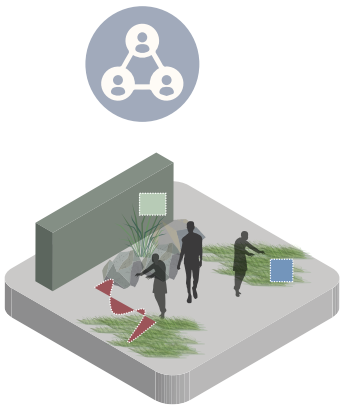

Social

Process of story-telling $3=$ Naming

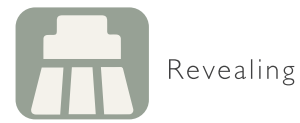

Landscape narrative

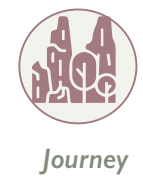

Gathering

Opening

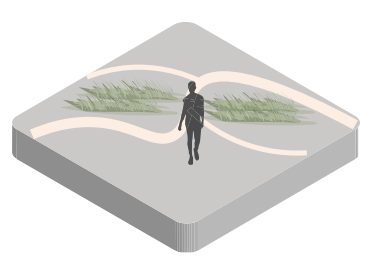

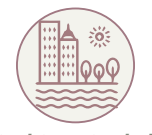

Remain historical things/

Cultural significance 


\section{SITE}

Site Analysis 


\section{VALUES}

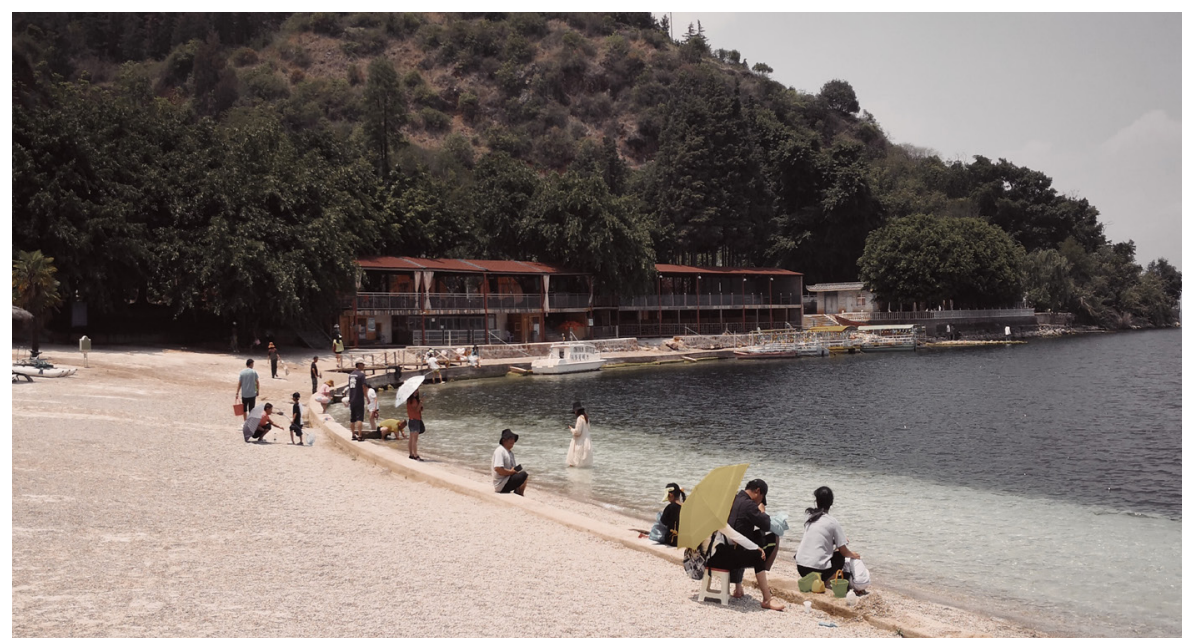

figure 79. Lake Fuxian

CONTEXT

SITE SCALE

CLOSER SCALE 


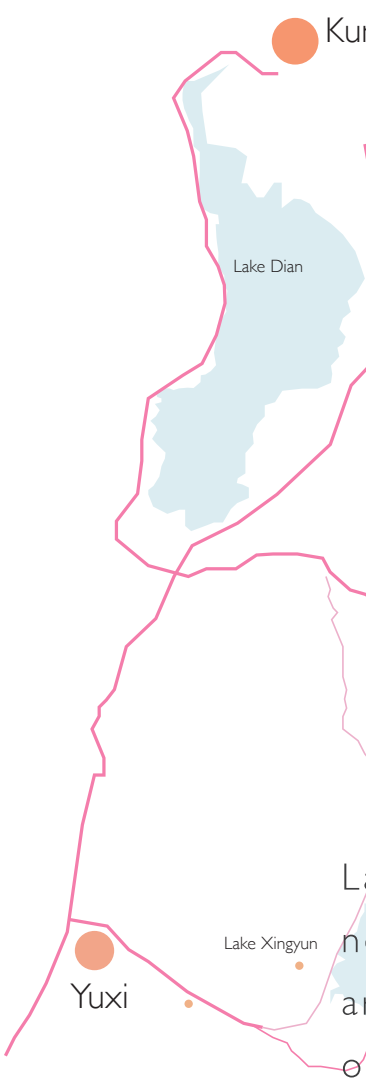

figure 80 . Location of the lake

\section{Journey of Lake Fuxian}

Lake Fuxian has over seven million visitors all year round, directed from two neighbouring cities, Kunming and Yuxi (Yuxi News, 2017). There is no airport around the Lake, the closed airport is in Kunming City (figure 80), which is one hour and half drive away. Most of the visitors are come within Yunnan Province, and they come to Lake Fuxian by cars. So, the public transportation in Lake Fuxian is relatively poor, without driving there is no visitor access to the Lake. The reason visitors come to the lake because the lake is considered one of the most beautiful lake in China. Some people are aware of the stories related to the about underwater building remains from Dian culture, and came to the site to explore the structure further. According to the data analysis, after a documentary about the underwater structure in Lake Fuxian was released, it attracted at least one million visitors (Yuxi News, 2017).

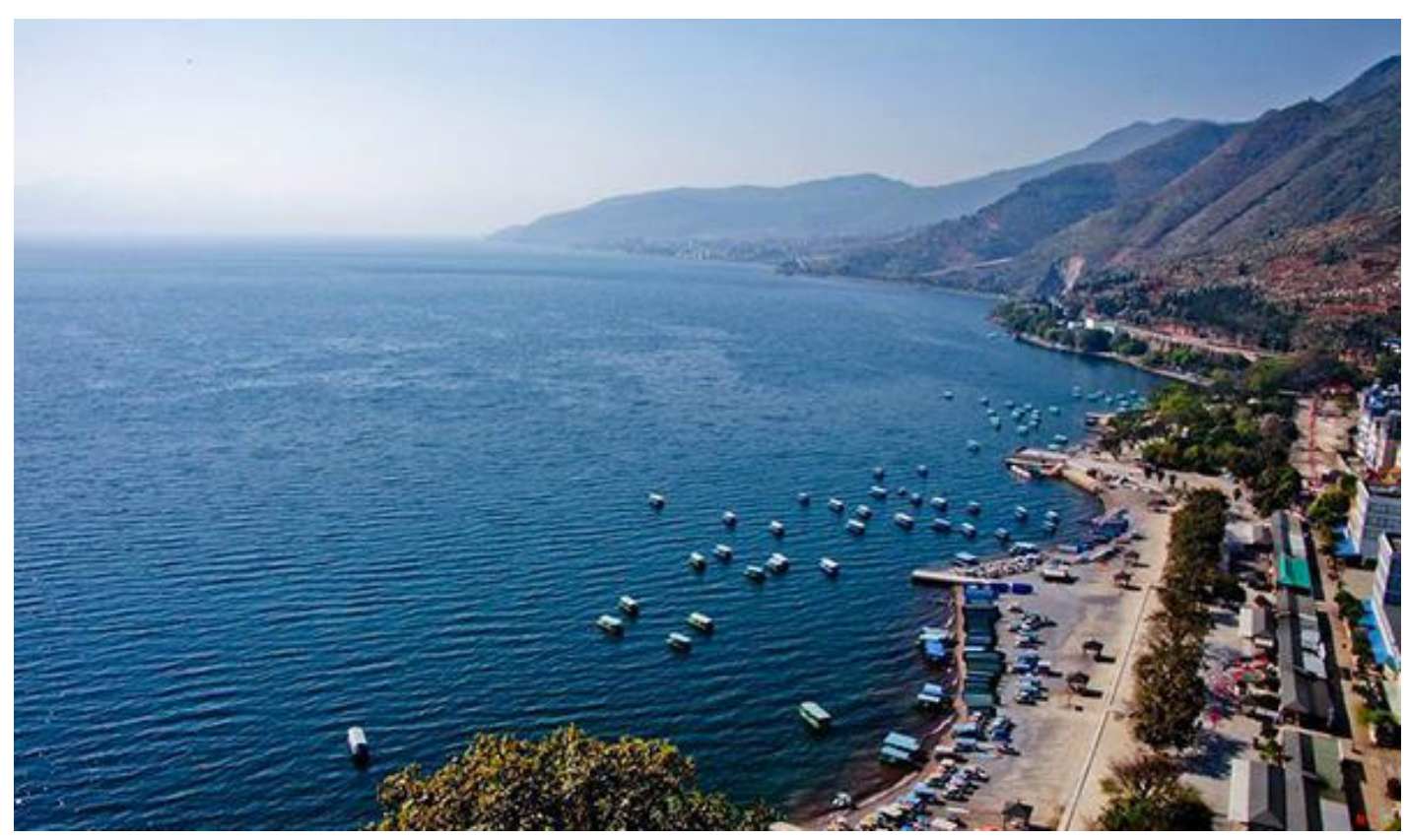

figure 81 . Bird view of Lake Fuxian

(The buildings in this figure has been removed) 
There are six types of sediments, in and around the lake (Chen, 2015), they alter the colour of the water due to changes in weather conditions and seasons.
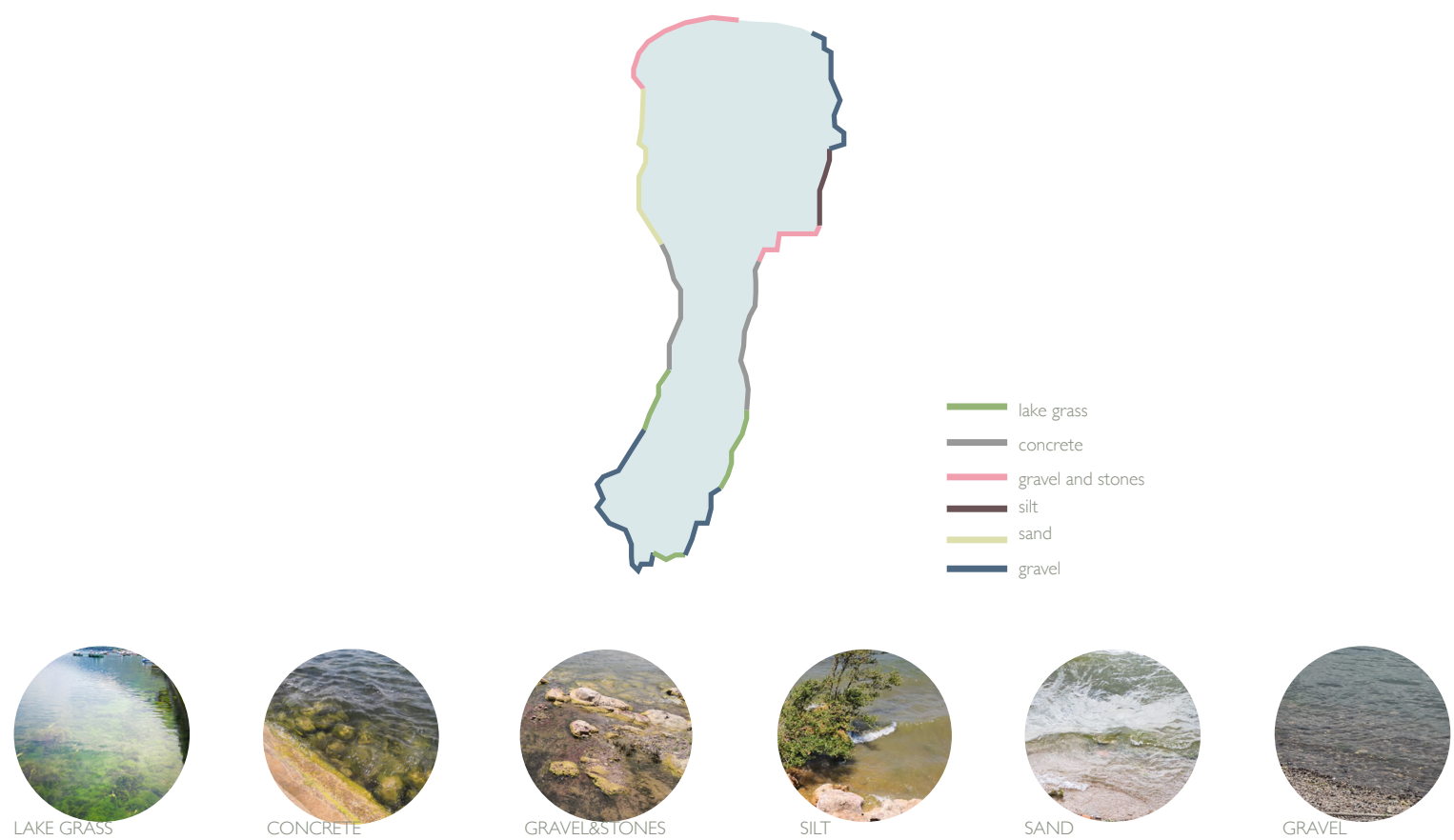

\begin{tabular}{|l|l|l|l|l|l|}
\hline Name & Visibility & \multicolumn{1}{|c|}{ Uses } & Colour & Texture \\
\hline Gravel \& stones & $60 \%$ & Interact with water & Grey, Green & Smooth, Scratch Skin & Change during rainy day \\
\hline Lake Grass & $90 \%$ & Have a clear view of water & Blue, Green & Slippery,only watch & Crystal-clear all the time \\
\hline Sand & $40 \%$ & Interact with water,with a lot of potential & Yellow, Green & Safe to play & Change during rainy day \\
\hline Concerete & $95 \%$ & Have a clear view of water,touch water & Blue, Green, Yellow & Slippery & Crystal-clear all the time \\
\hline Silt & $20 \%$ & & Green, Yellow & Slippery, Unclean & Change during rainy day \\
\hline Gravel & $20 \%$ & Interact with water,with a lot of potential & Grey, Blue & Scratch Skin & Crystal-clear all the time \\
\hline
\end{tabular}

figure $\mathbf{8} \mathbf{I}$. sediments on lakeshore diagrams 


\section{Pollution details}

\author{
Lake Fuxian \\ Water Quality: \\ Class I water quality: good water quality. \\ only needs disinfection, and surface \\ water can be used for drinking after \\ simple purification (such as filtration) \\ and disinfection; \\ Class II water quality: slightly polluted. \\ Regular purification treatment can \\ provide drinking water; \\ Class III water quality: it is applicable \\ to the secondary protection zone of
}

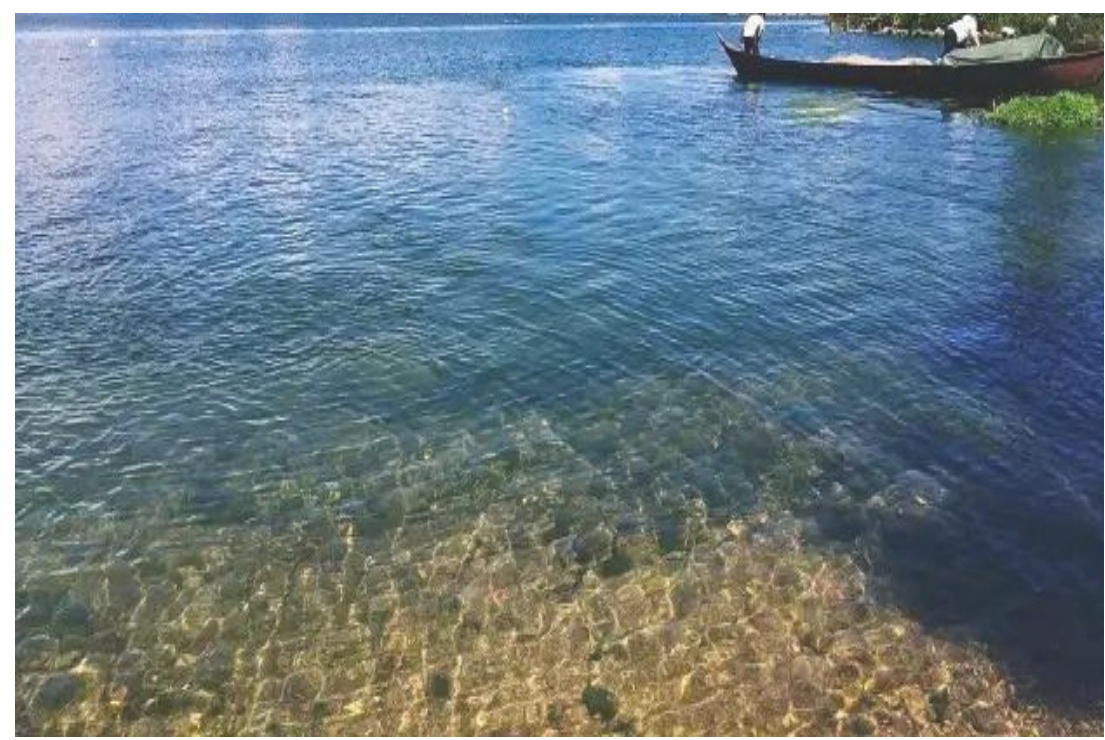

figure 82. Photo of Lake Fuxian centralized drinking water source, general fish protection zone and swimming zone;

Class IV water quality: suitable for general industrial protection areas and not in direct contact with human body;

Class $V$ water quality: The water bodies that exceed the five water quality standards basically have no use function (China water quality standard, 20|8).

The current water quality of Lake Fuxian is Class I. There are two nearby lakes to Lake Fuxian: Lake Dian and Lake Xingyun (figure 83). Both of the lakes have been polluted due to urbanization and soil erosion with Grade IV (Xinhua, 2020). 
Also, the total amount of water resources in Lake Fuxian accounts for $9.16 \%$ of the total freshwater resources of lakes in China(Dai et al, 20l6) As a Lake contained almost one tenth fresh water in China, the government decided to use severe methods to manage the lake water.

For the protection of Lake Fuxian, the government of China decided to move all the residents away from the lakeshore(figure 84). Also, to reduce the impact of farmland on the quality of water, the government prohibited any related activities to agriculture or pasture from around the lake (KunmingNews, 2019). Besides, the outlet of the lake, also known as the Ge River(Connection between Lake Xingyun and Lake Fuxian), flowed into Fuxian Lake until recently; it has been closed off to protect Fuxian Lake from the polluted waters of Xingyun Lake(Dai et al, $20 \mid 6)$.

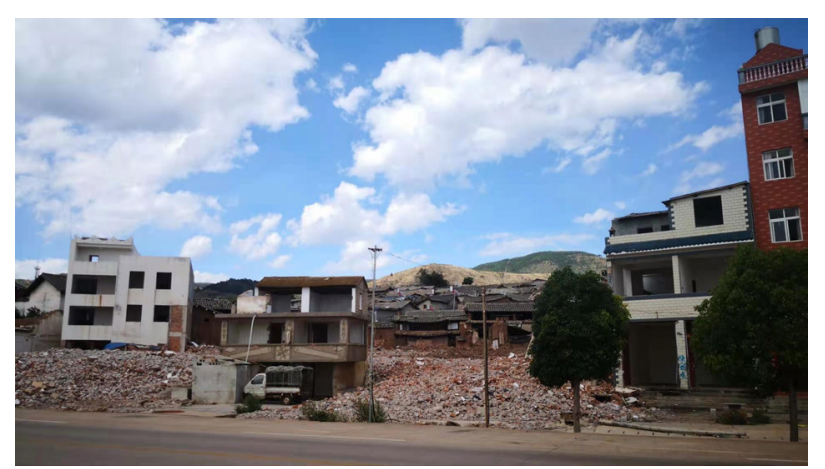

figure 83. Ruined buildings, photo took in 2019

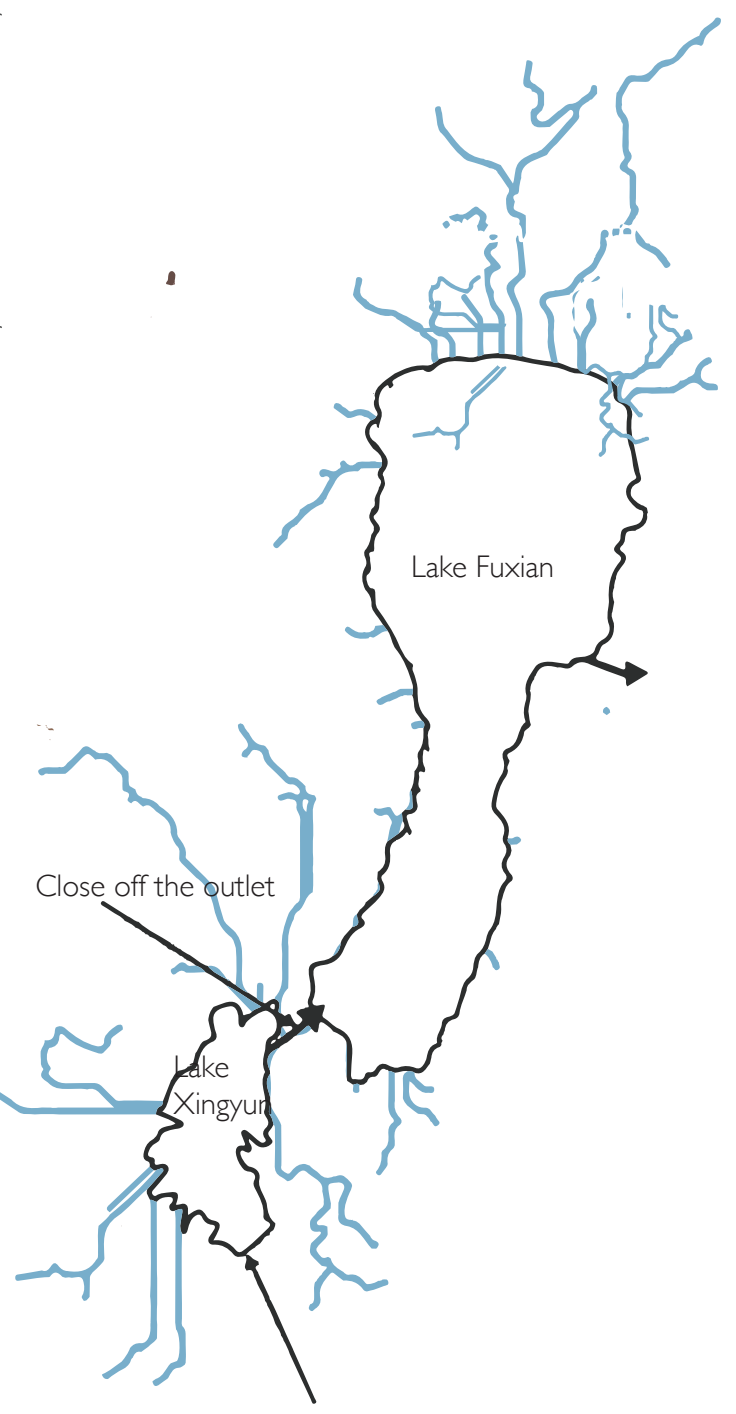

figure 84. close off the outlet of the connection between Lake Xingyun and Lake Fuxian 


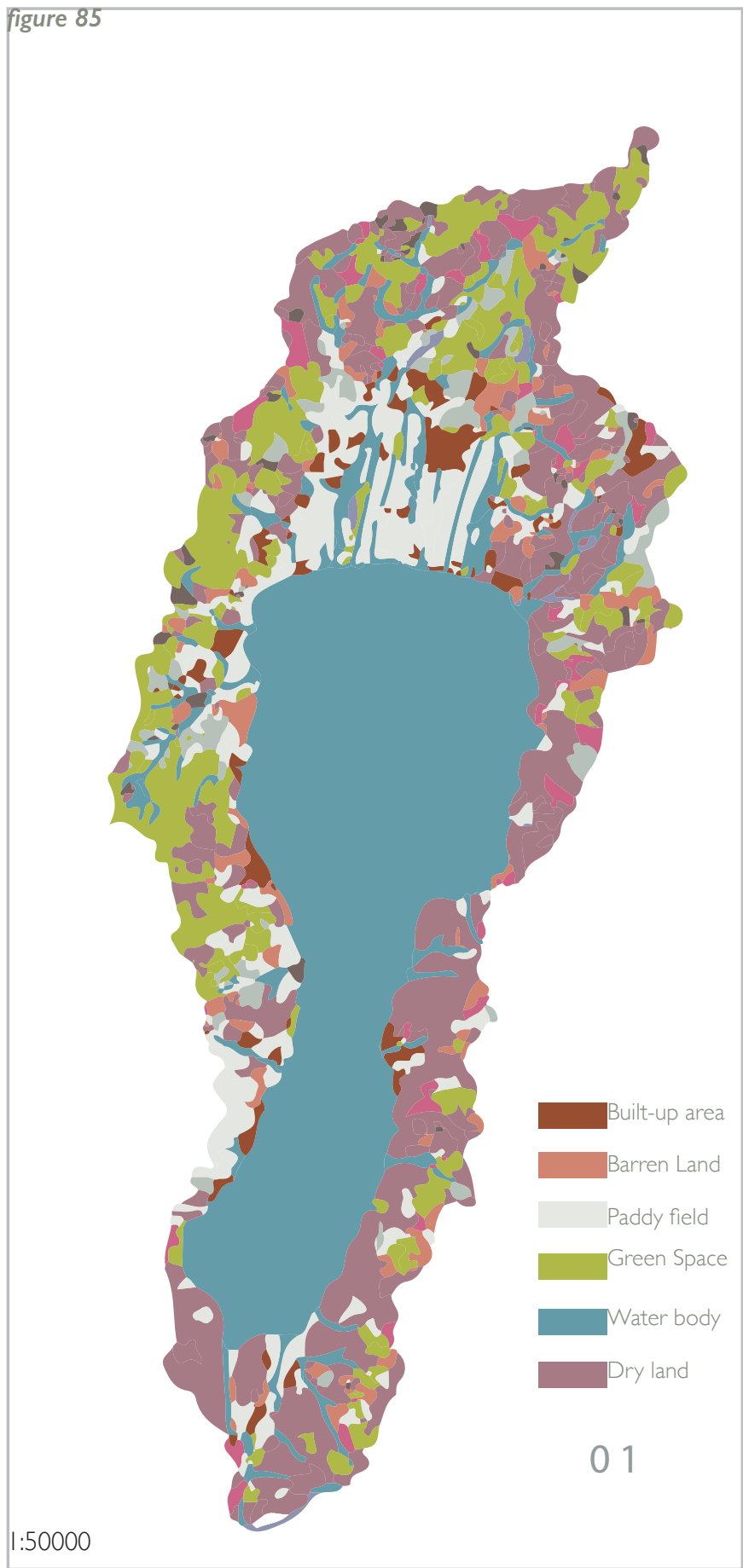

Lake Fuxian is a vital fishery base and scenic spot within the Yunnan Province. However, due to the species invasion and large human recreational fishing, many native fish species are endangered (total 40 species, 12 species endangered). For protecting native fish, the government prohibited any fishing activities on the Lake(KunmingNews, 2019).

Land use 


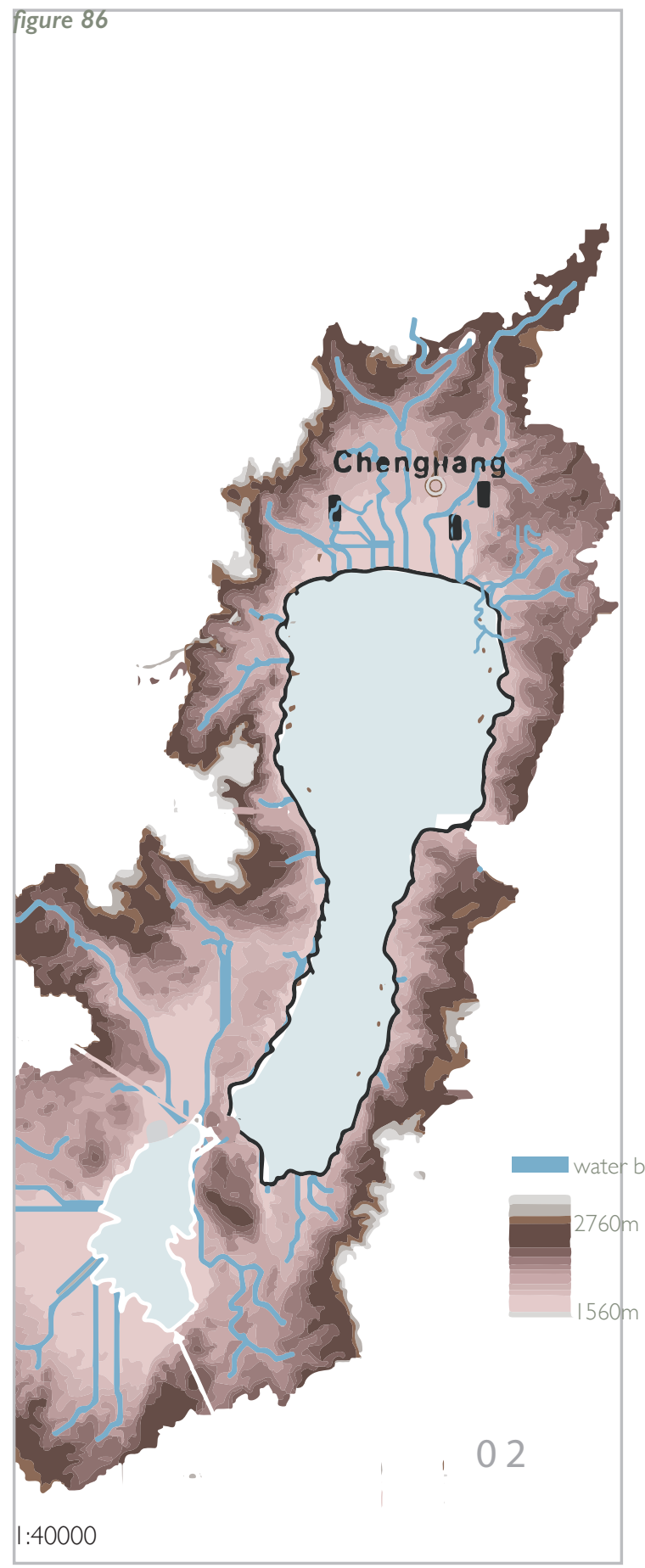

Inlets of the lake

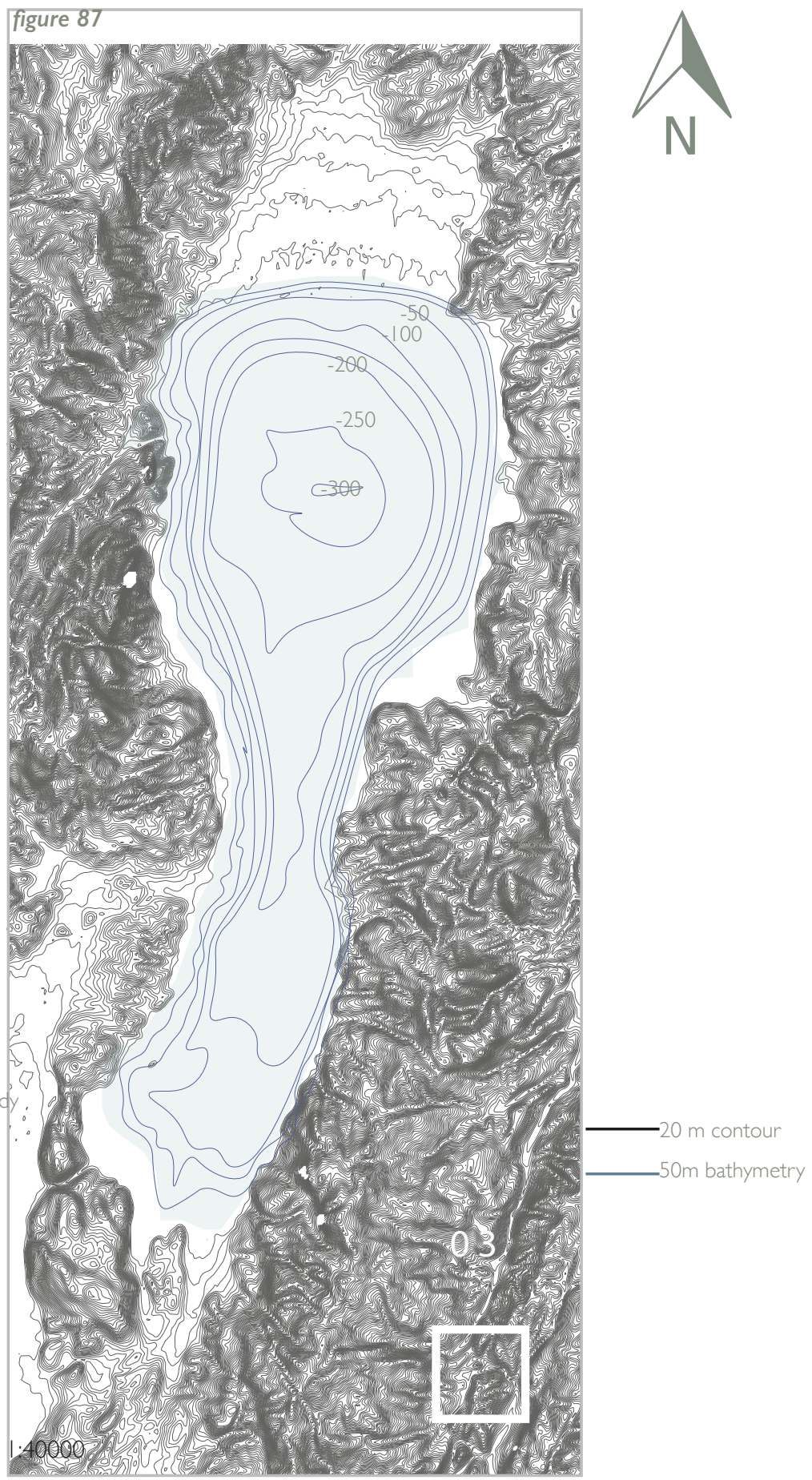

Topography

$25 \mathrm{~m}$ contour of Lake Fuxian 


\section{PLANTS AROUND THE LAKE}

Pollution has been, and continues to be, a major issue at Lake Fuxian. The main reason for this is the sewage and waste runoff, leading to a high pollution load of nitrogen and phosphorus in the lakeshore (Yang, 2018). The first step that the introduced by the government was to demolish all residential buildings, including hotels, around Lake Fuxian(KunmingNews, 2019). Additionally, to help aid the lake in its recovery, the government has already constructed a wetland on its northern side, with the intention to later expand it(KunmingNews, 2019)

figure 88. Plants in Lake Fuxian
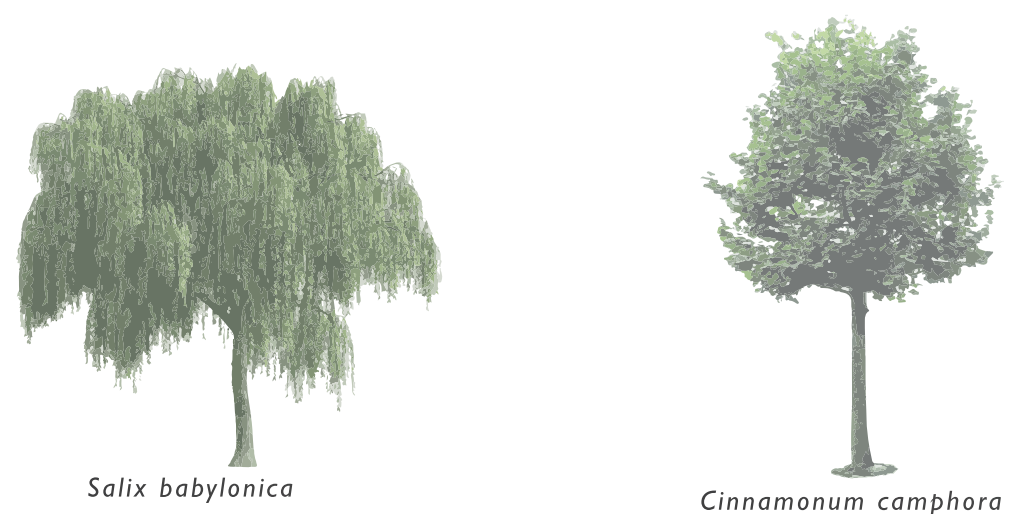

Babylon willow

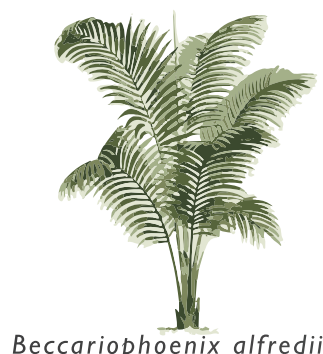

camphor tree palm

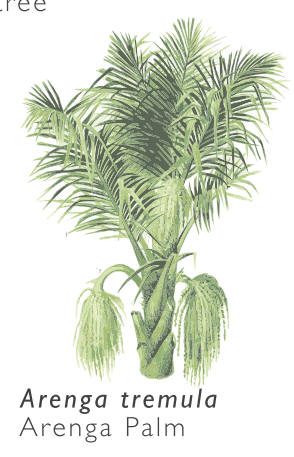


Lake Fuxian can be divided into three vegetation groups, deciduous broad-leaved forest, evergreen broad-leaved forest and wetland grass(fig) (Yang, 2018). Lake Fuxian plants include Fabaceae Family, Palmae Family, Moraceae Family, Lamiaceae Family, and Poaceae Family(Yang, 20|8). Furthermore, the establishment of the northern wetland provides an addition of over 100 plant species (Yang, 20l8). The most common plants include invasive plants, which are shown in the above figures.

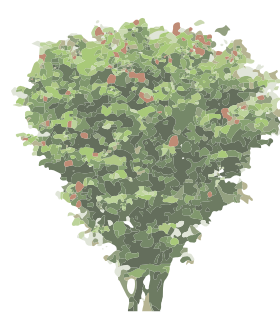

Morus alba white mulberry

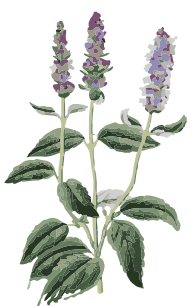

Stachys affinis Chinese artichoke

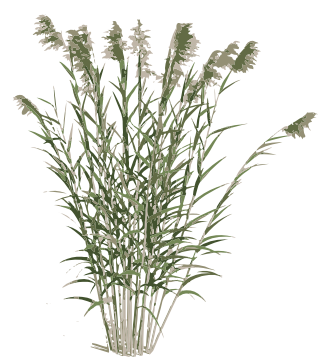

Phragmites australis

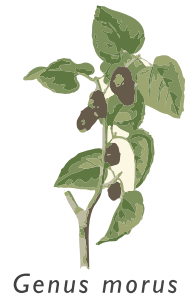

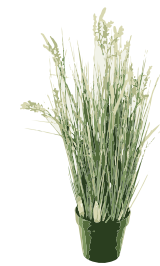

Scirpus validus grey club-rush

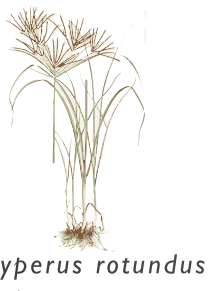

nut grass 


\section{SITES}

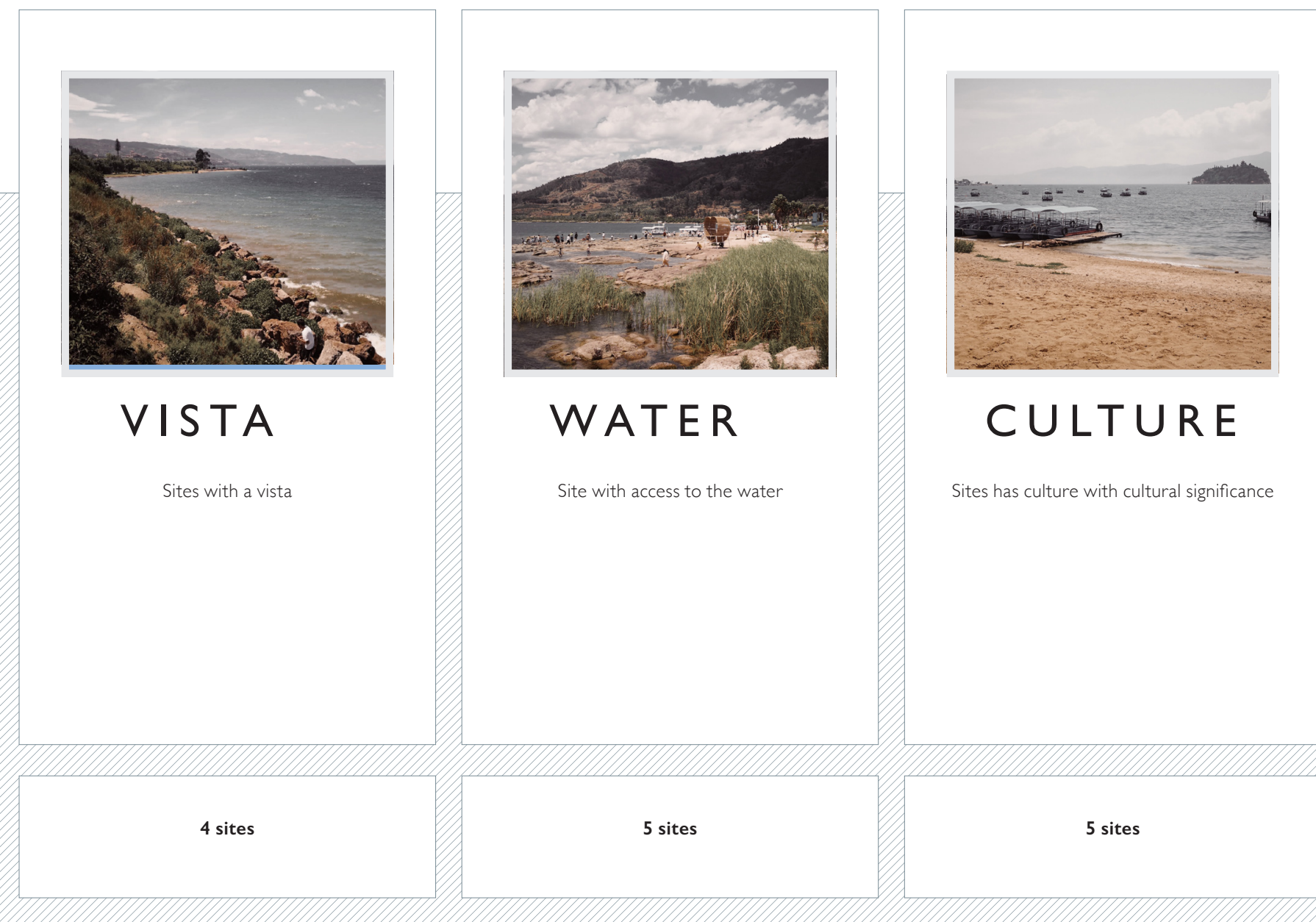

figure 89. Lake Fuxian

There are 14 sites around the lake that allow people to partake in different activities. The 14 areas can be divided into three groups: the sites that can see the lake, the sites that have access to water and the natural environment, and finally, sites with vital cultural significance. 
There are several sites(more than 15) around the lake and allow people close to the water. Most of the visitors come to the site by driving a car. Then, consider about the convenience about the visitors, and possibilities to analyse what people was doing on the sites. So, the all of chosen site for analysis allow people park a car, and then walk to the water or have a lake view.

This research will complete a detailed analysis includes visualisation, natural environment, gathering space and cultural significance for each of these sites, as a way to determine potential locations for designed development. 


\section{SITES WITH A VISTA}

\section{Site Values:}

\section{visualisation:}

has mountain view without any trees hide the views 30

has mountain view but hidden by trees 20

has open water views 15

has lake views but hidden by trees 10

has lake views but most area has been hidden 5

natural environment

massive planting 25-30 (according to the density of plants)

only a few trees 5-10

has ecotone 10

gathering space:

has different types of people use it 15

has most of the people use it 20

has few people use it 5

only young people use the space 5

cultural significance:

has mountain views 10

has a little bit culture remains 10

Used to settle by Dian history 25

has fossils 30

With Dian legend 30

water activities:

can see the lake with long-distance 5

can see the lake closely 10

can touch the water $2 Q$

has different types of water activities

can take a boat 5
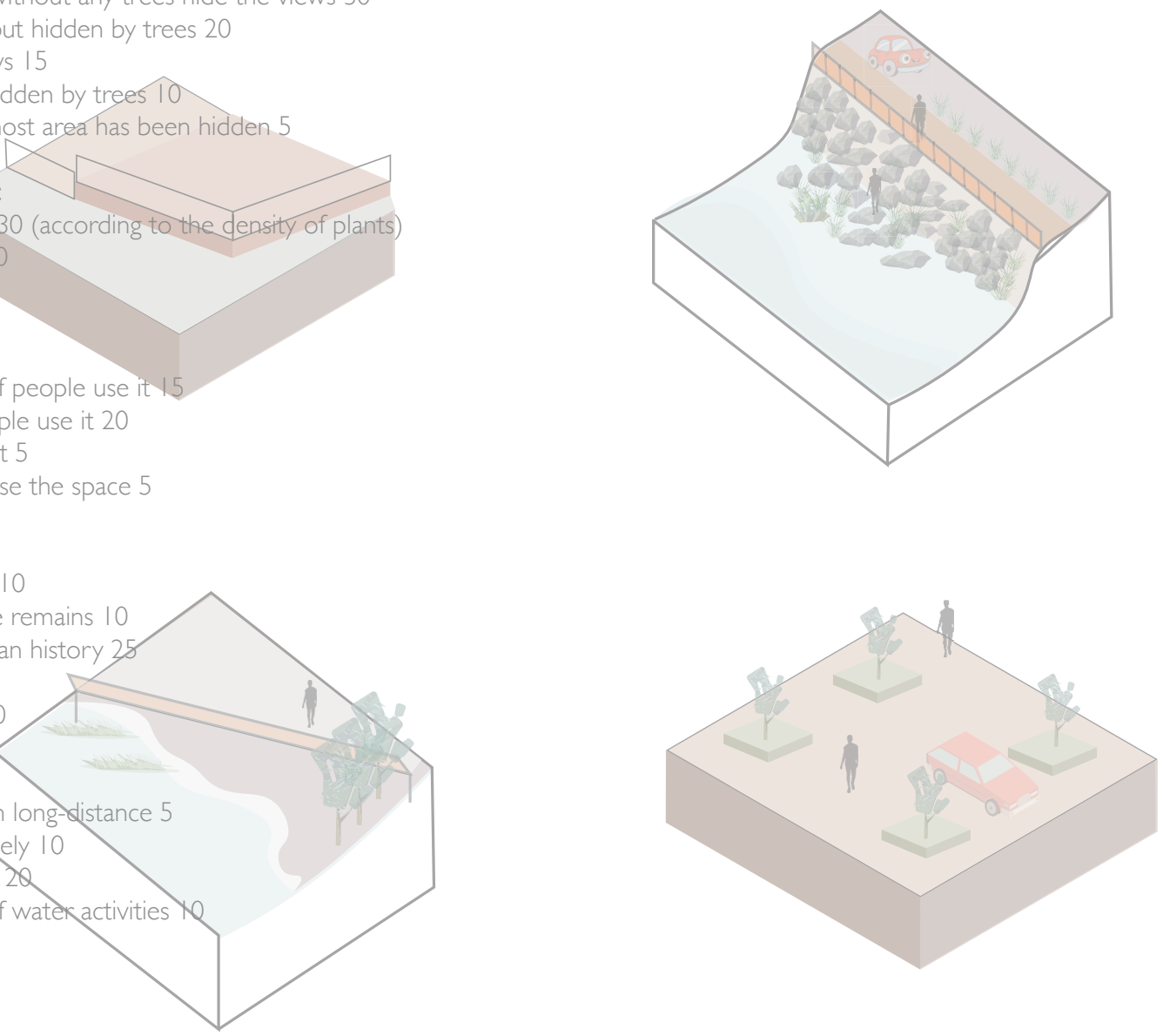


\section{SITE I.I}

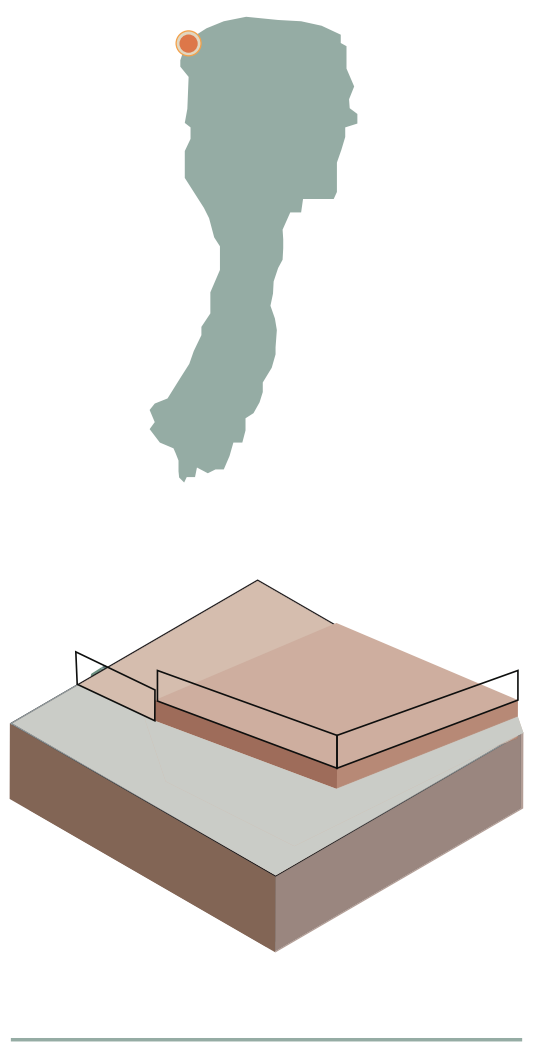

DATA ANALYSIS

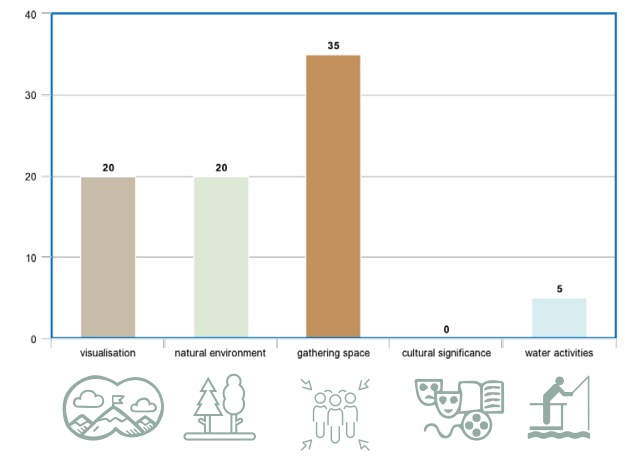

from top to bottom. --figure92 9394

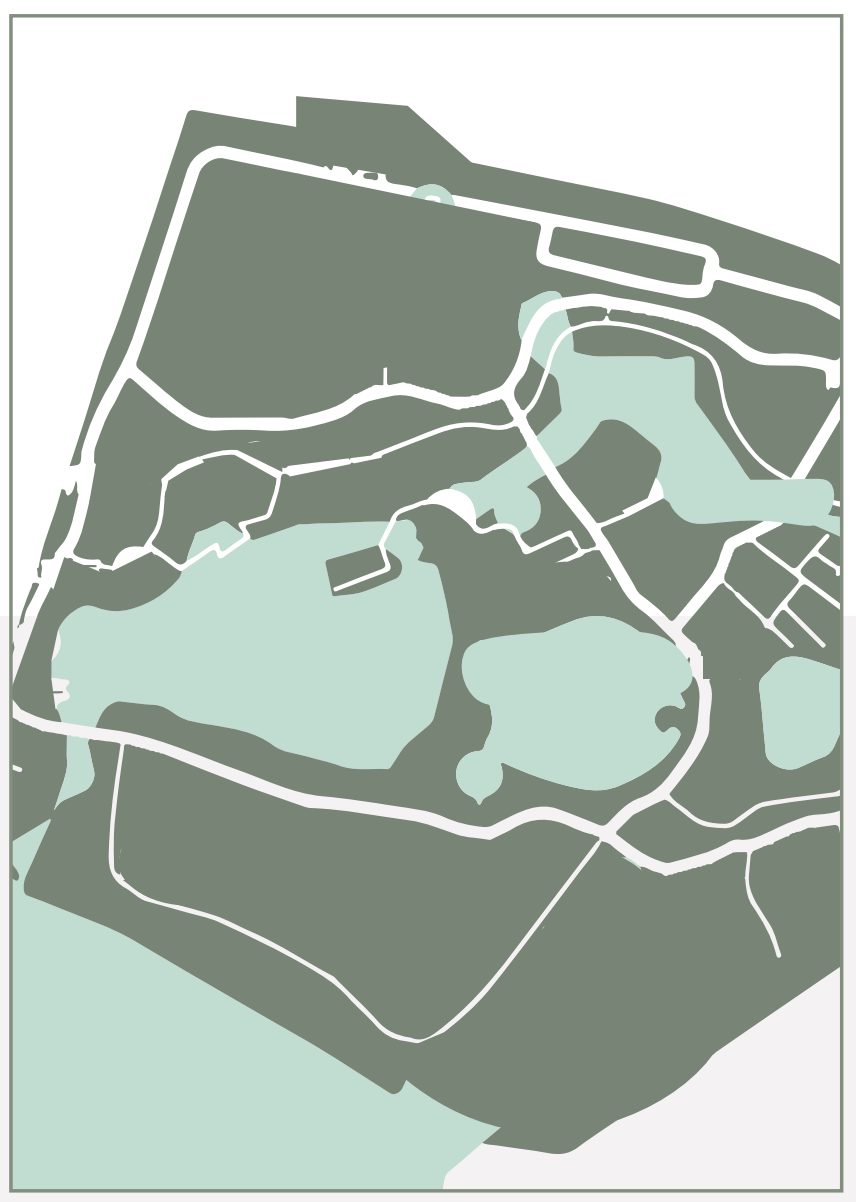

SITE I.1

WETLAND PARK

The site is a wetland park at the northern section of the Lake. The community built it in 2018 , so it is a fairly recent development (ctrip, n.d.) This is a very active section of Lake Fuxian as it contains many restaurants that attract a lot of visitors all year round.

However, the tall wetland plants around the park only provide around 10 metres of visibility to the lake (figure 96). The site topography does not facilitate for a higher vantage point. 


\section{- - num}

Scirpus validus $f$

Typha orientalis Presl

Arundo donax var

Thalia dealbata Fraser

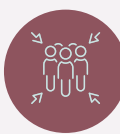

\section{GATHERING SPACE}

Many people gathered here because it is a new-built- park with fresh look. The wetland design does not have a space to allow people to touch the water or do any water activities.

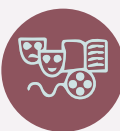

\section{CULTURE SIGNIFICANCE}

There is no existing culture, or any history people can discover about the site. The wetland design does not provide permanence to the water's edge and therefore lacks physical and visual connection.

mainly conformed into a lookout over the lake (Fig).
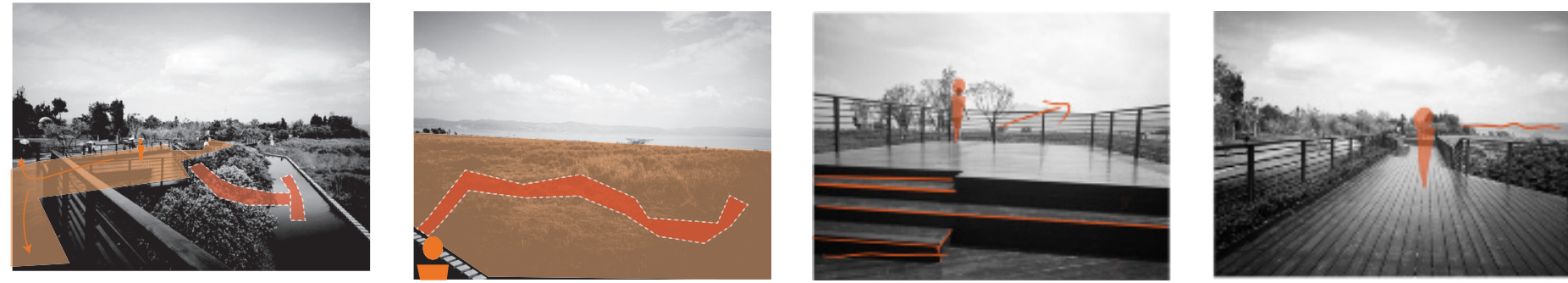

figure 95 , photos of the site

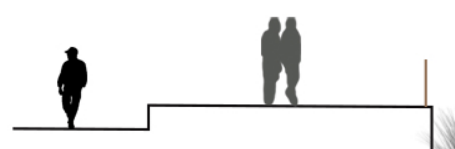




\section{SITE I.2}

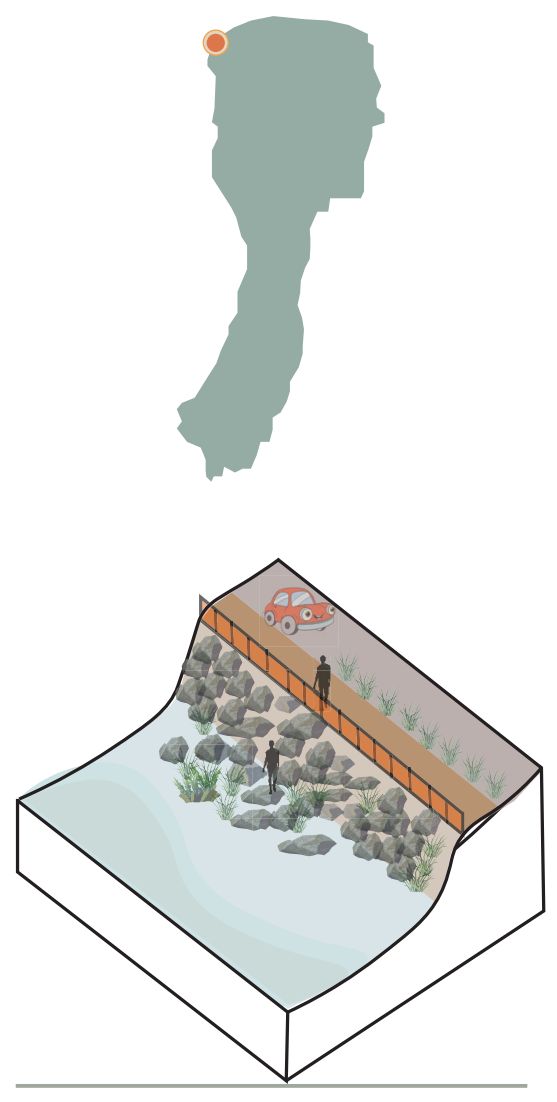

DATA ANALYSIS

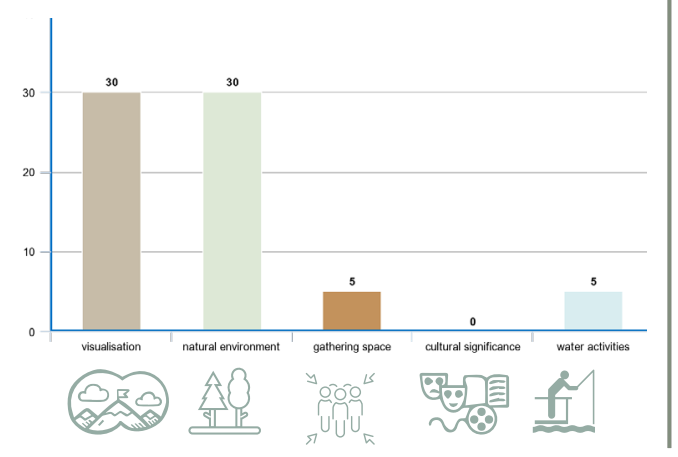

from top to bottom. ---figure97 9899

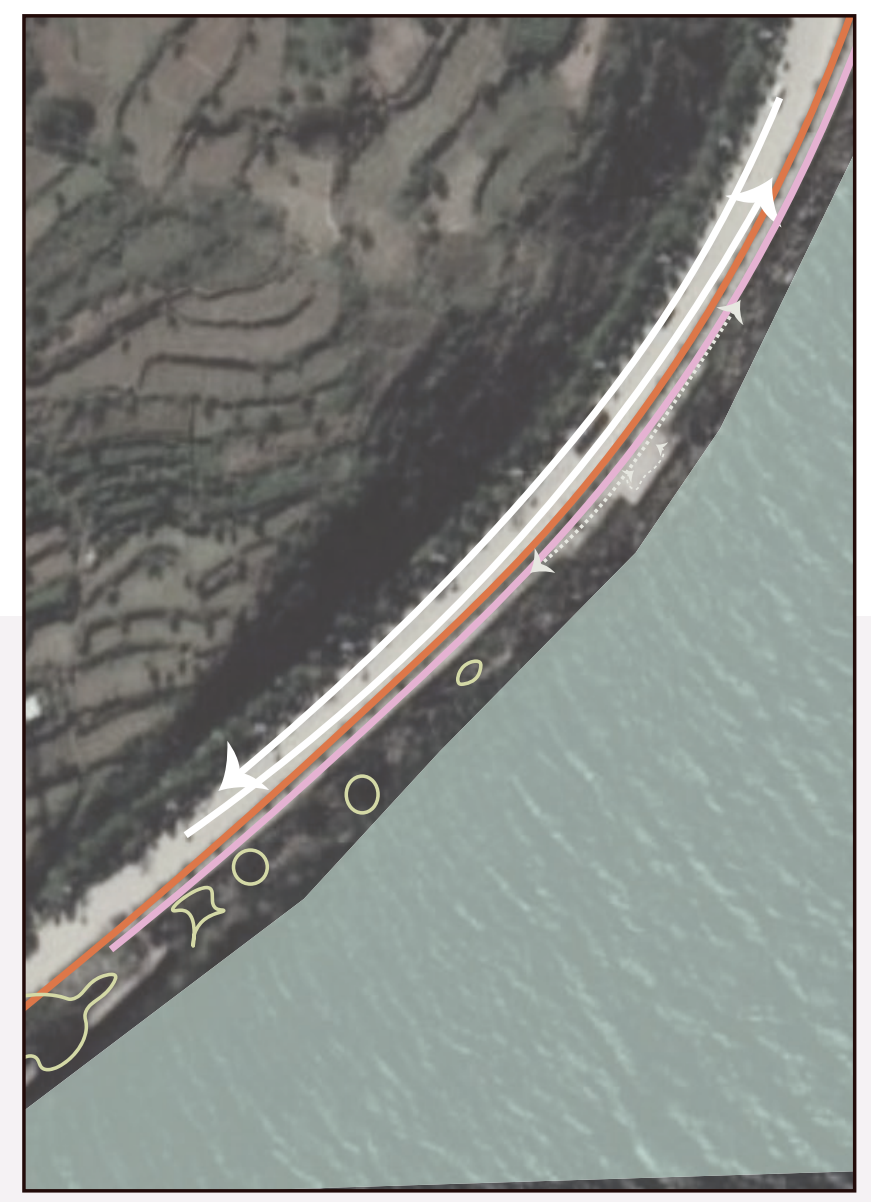

SITE 1.2

LOOKOUT

Similar to site I.I, this site is a viewing lookout to the lake. People park their cars on the road and walk straight to this lookout(figure 100).

A major setback for this site, is that people cannot get close to the water.Visitors will often climb the fence and travel over a series of stones to get to the water's edge. It is a dangerous behavior, as the path is treacherous, it could cause an accident.

To further aid in the sedimentation process, weeds and small plants grow in the gaps between the stones. However, the weeds are exotic plants and negatively affect the growth of the native vegetation. 


\section{- -}

Ageratina adenophora (Invasive species)

Agave tequilana

Cnnamonum camphora

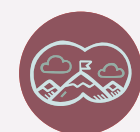

VISUALISATION

The lookout only has a view large of 8 meters towards the lake. However, the lookout is located on a high topographical area. By comparing with site I. I's perspective, the lake view of this site is better.

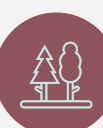

NATURAL ENVIRONMENT

There are many stones on the edge o lake, it is an excellent prevention to the site's sedimentation problem.

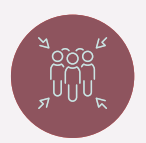

GATHERING SPACE

As the site only offers a lookout, visitors only feel the need to stay for around ten minutes.

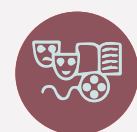

\section{CULTURE SIGNIFICANCE}

Same with site I.I, there is no existing culture or any history people can discover about the site.
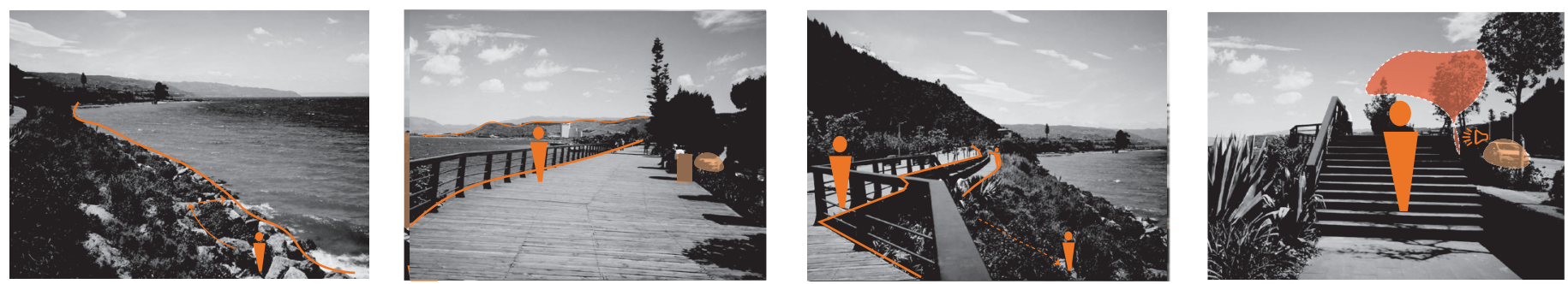

figure 100 , photos of the site 


\section{SITE I.3}
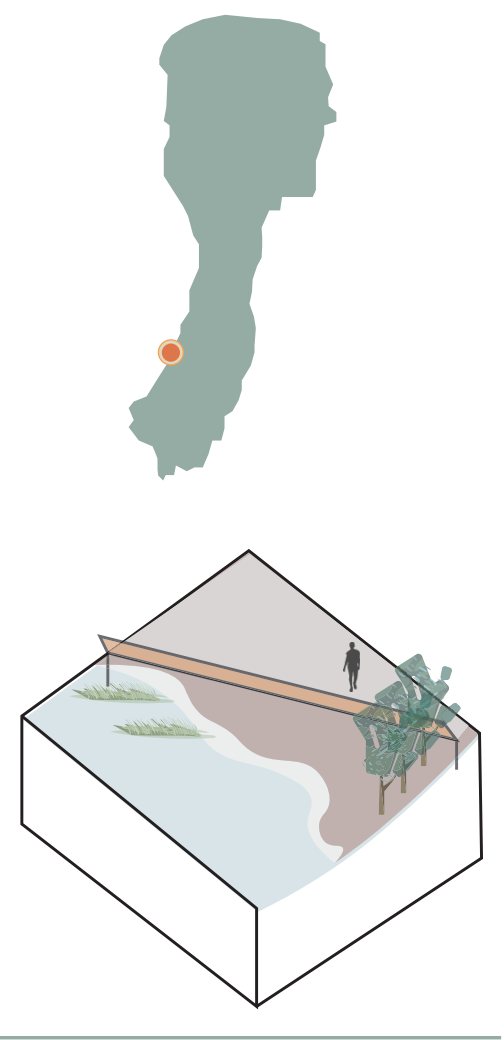

\section{DATA ANALYSIS}

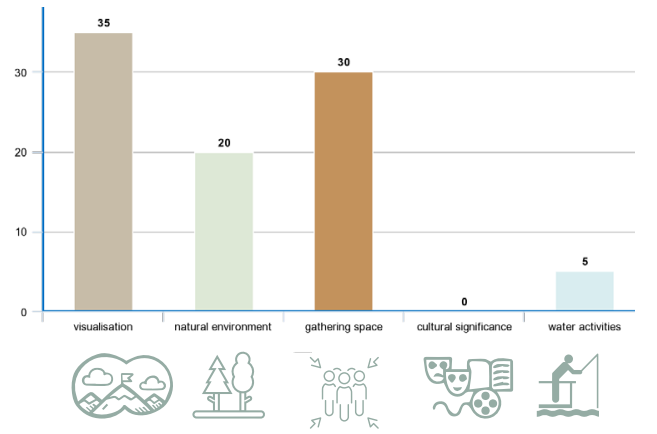

from top to bottom.--figurel $02 \quad 103 \quad 104$

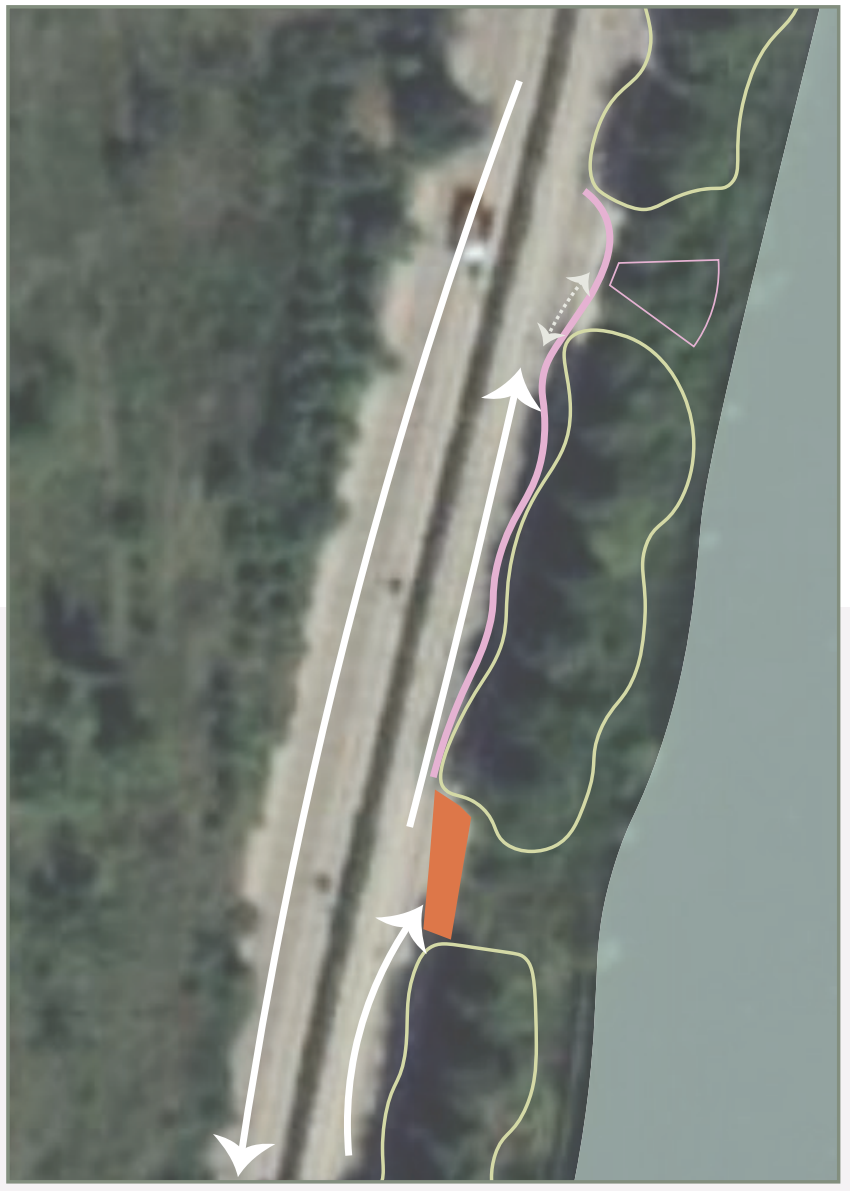

SITE 1.3

LOOKOUT
Same with site 1.2, this site is a lookout that lets people conveniently park their car and have a look at the lake. The lookout is close to the water; people are only two meters away from the water. The site is the most popular lookout around the lake because people from Jiangchuang County have to use this route to arrive at Lake Fuxian. There is signage on the road to let people know there is a lookout ahead.

The fence's shape is oblique to make users psychologically feel they are much closer to the water.The sediments around the site are mostly stones, so the lake looks transparent and clear. Nevertheless, the lake is too deep in this area, so the designer prevents people from physically touching the water as a safety precaution. There is also a sign of letting people do not close the water. Signage also acts a secondary measure to enforce this. 


\section{- - num}

Salix babylonica

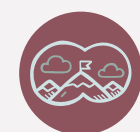

VISUALISATION

Proximity to the water is around two meters, it allows visitors

to feel physically connected

to the water without actually

interacting with it. However,

few trees on the lakes edge

block the parts of the view from the lookout.

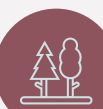

NATURAL

\section{ENVIRONMENT}

Three Salix babylonica trees are growing on the edge of the lake

they are 3 meters tall and block the view to some parts of the lookout.

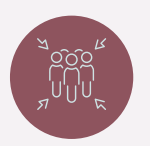

GATHERING SPACE

Due to most visitors going to the Lake from Yuxi City, this route is popular for tourists and travellers have to through the site, many people gathered in this site.

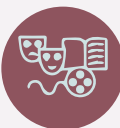

CULTURE SIGNIFICANCE

There is no existing culture, or any history that people can find about the site.
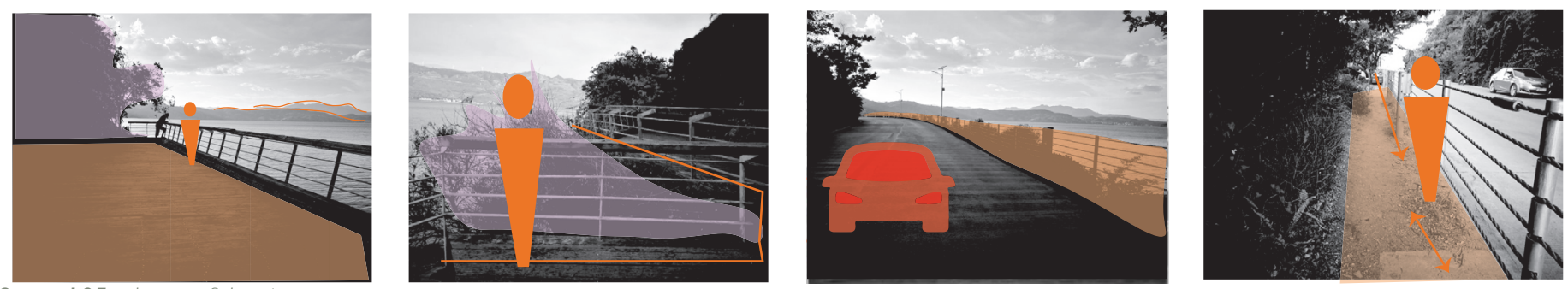

figure 105 , photos of the site

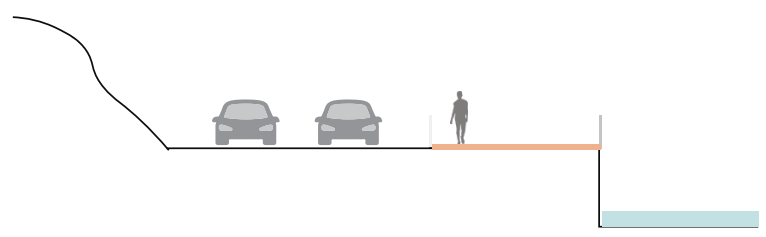




\section{SITE I.4}
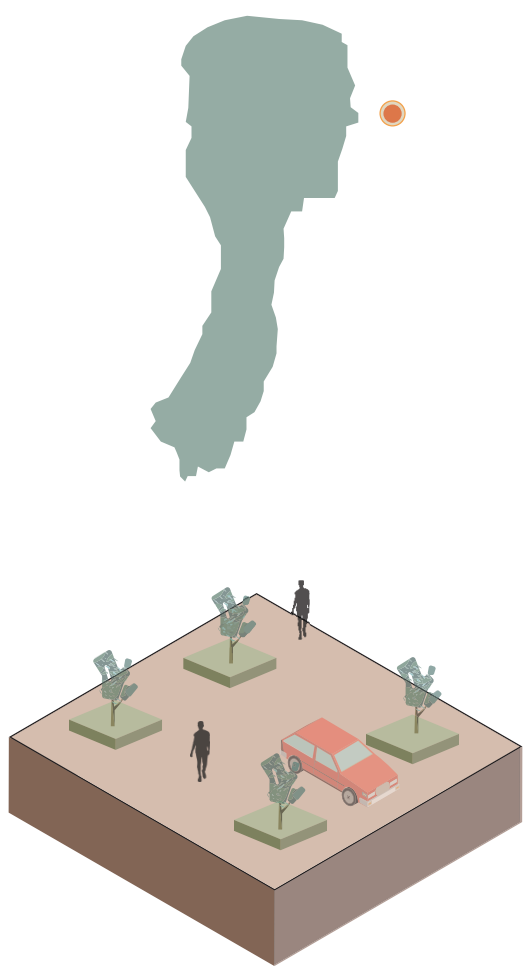

\section{DATA ANALYSIS}

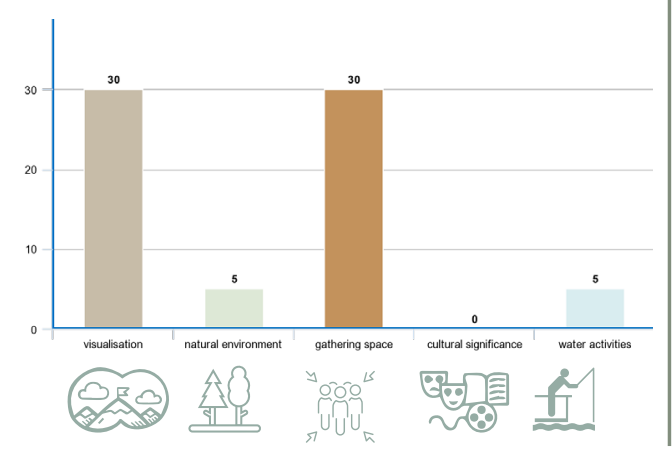

from top to bottom.--figure 107108109

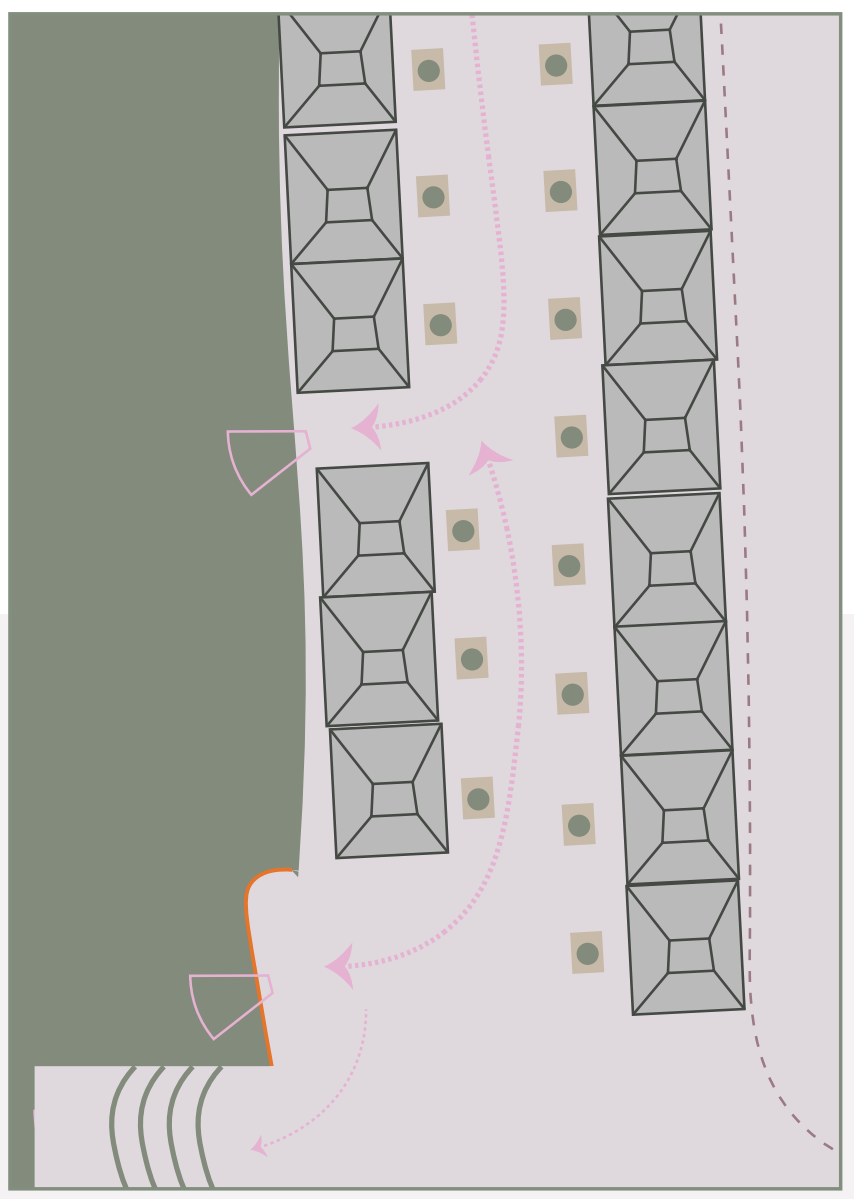

SITE 1.4

VIISITOR CENTRE

This is a newly developed community in 2017 , half of the site has been completed, with the rest still under construction. It is built on a mountain that is a ten minutes drive from the nearest lakefront. The site is very active, due to local businesses, such as restaurants, this location provides facilities for people to stay longer and enjoy their time. Architecture in this site looks different from traditional Chinese architecture. 


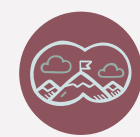

\section{VISUALISATION}

The lookout on this site provides sightlines to the Lake and

mountains. The topographical

elevation of this lookout provides excellent sightlines to the

surrounding mountains and entire lake. However, people prefer

to stay indoors due to a lack of sheltering space.

\section{会是}

대난

NATURAL

\section{ENVIRONMENT}

There are only few plants which

is seasonal flowers on the and

act as a decoration of the street.

\section{GATHERING} SPACE

The site is functional, with

a lookout to the Lake and

mountains, seats, and interior

space like Tea House or

restaurant. However, due to the

small size of the lookout, visitors

do not spend much time here

compared to the restaurants

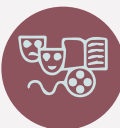

\section{CULTURE SIGNIFICANCE}

There is no existing culture, or any history people can find about the site. The site has beautiful mountain views which are essential elements of Dian culture.
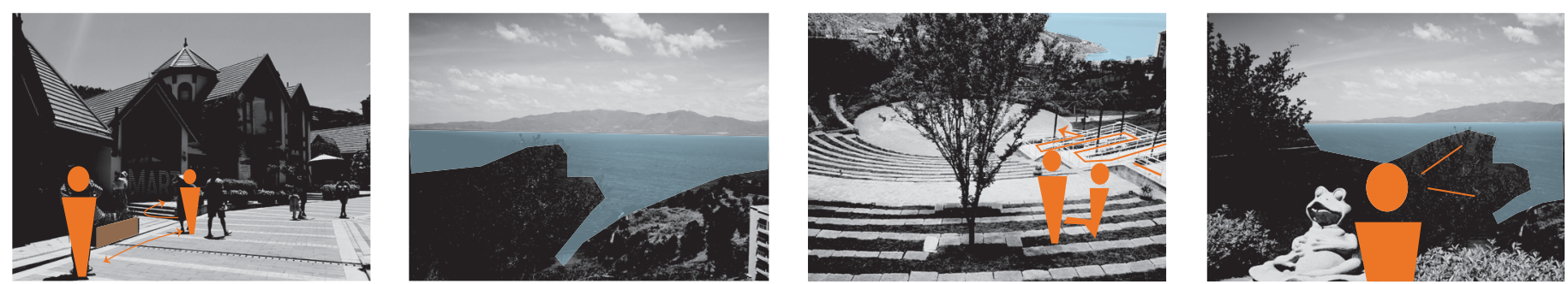

figure I I0, photos of the site

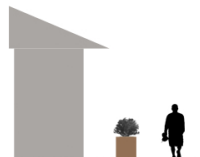




\section{SITES WITH ACCESS TO WATER}

\section{Site Values:}

\section{visualisation:}

has mountain view without any trees hide the views 30

has mountain view but hidden by trees 20

has open water views 15

has lake views but hidden by trees 10

has lake views but most area has peen hidden 5

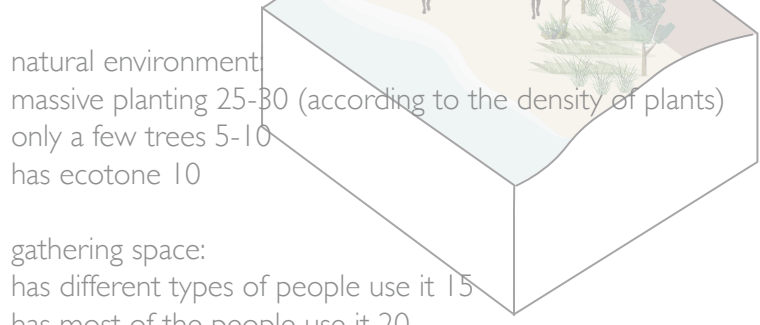

has most of the people use it 20

has few people use it 5

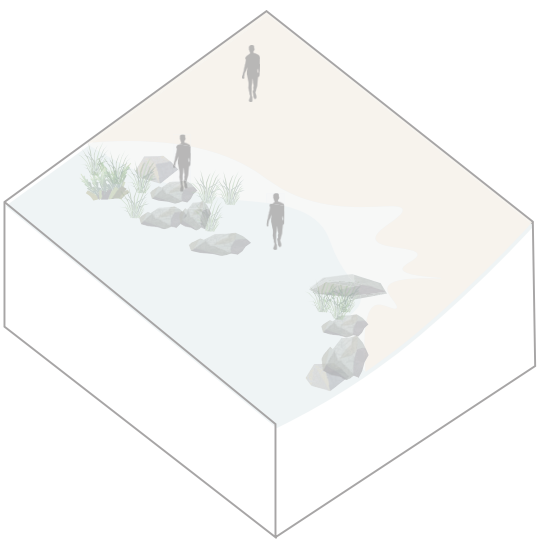

only young people use the space 5

cultural significance:

has mountain views 10

has a little bit culture remains 10 Used to settle by Dian history 25 has fossils 30

With Dian legend 30

water activities:

$$
30
$$

can see the lake with long-distance 5

can see the lake closely 10

can touch the water 20

has different types of water activities 10

can take a boat 5
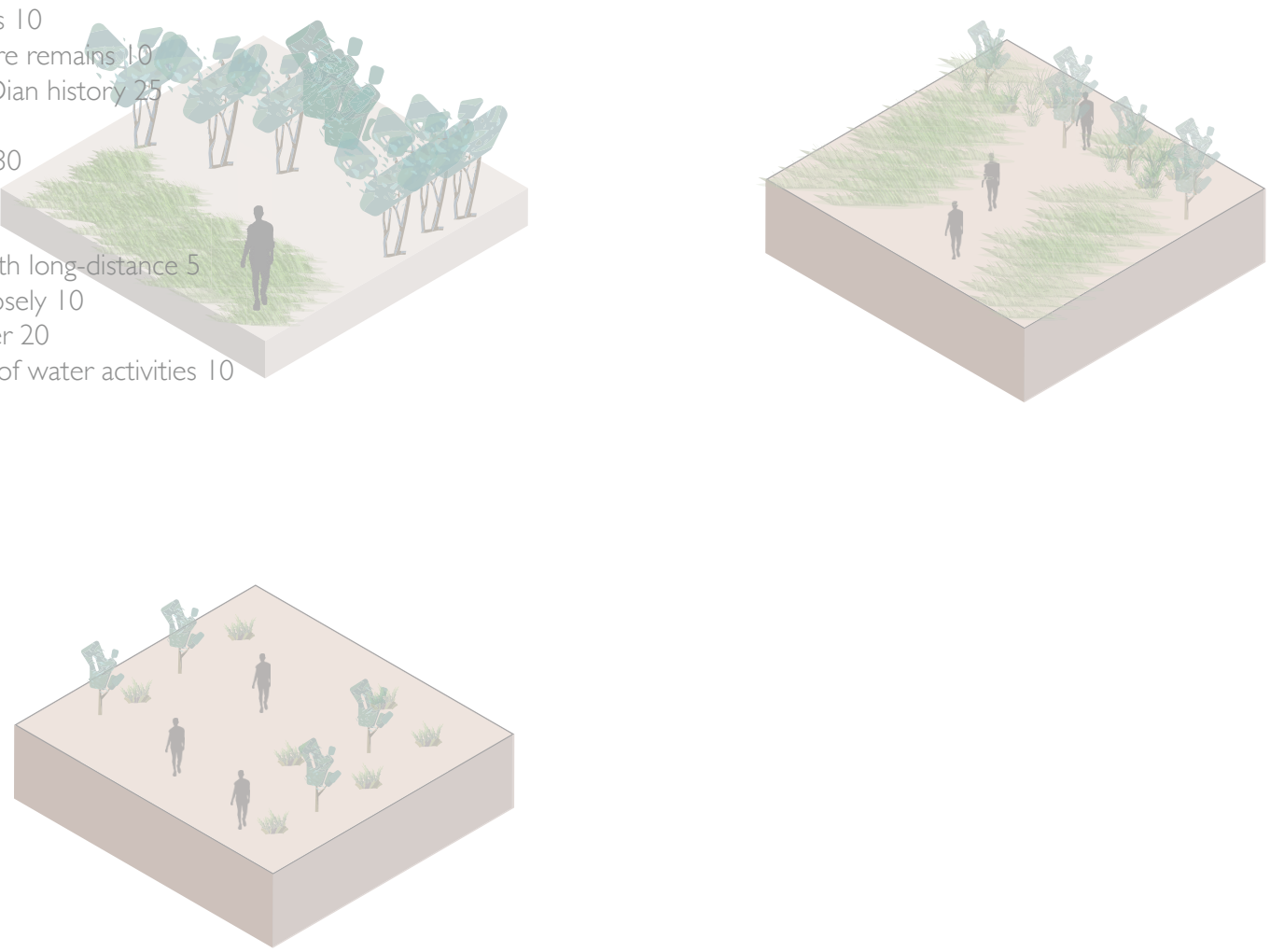


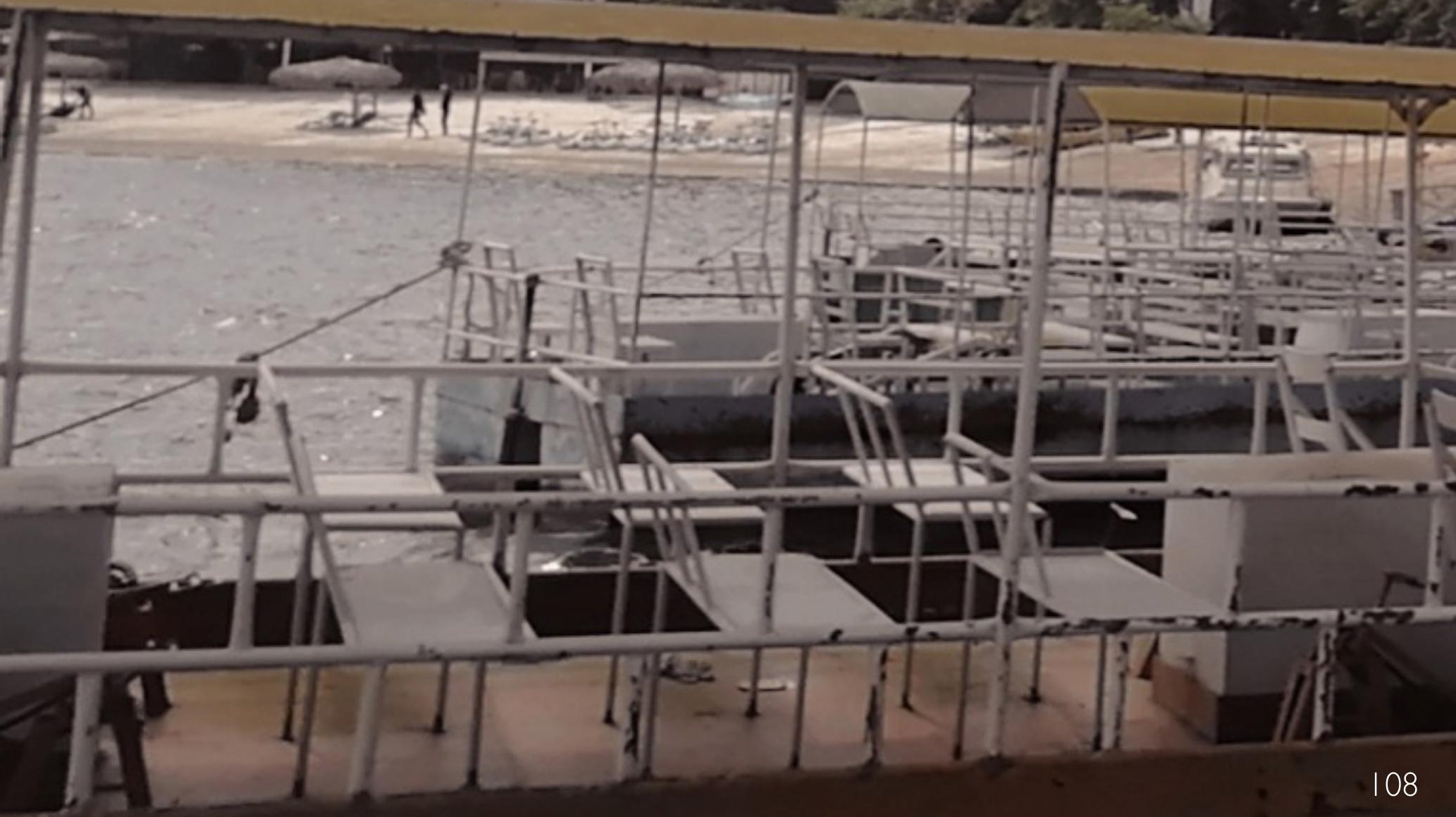




\section{SITE 2.I}
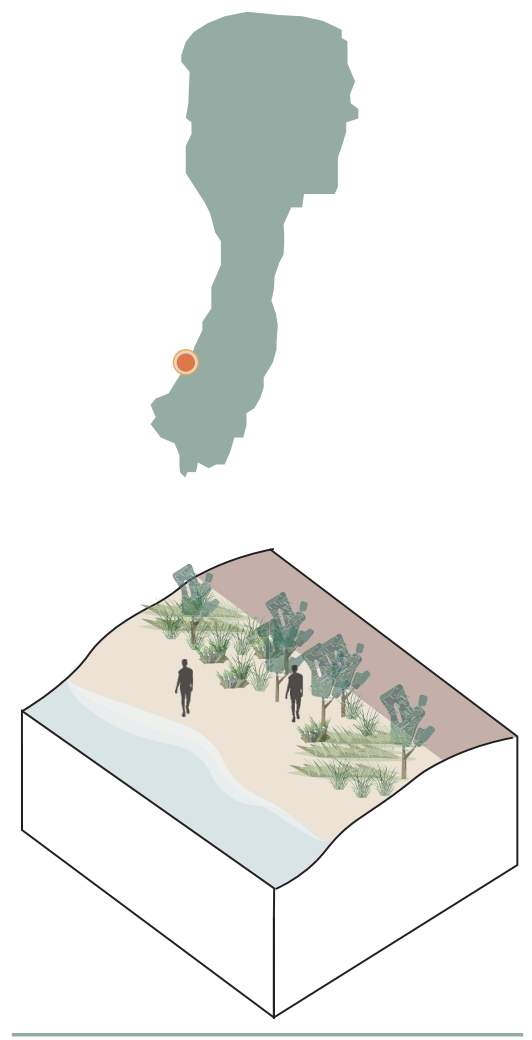

\section{DATA ANALYSIS}

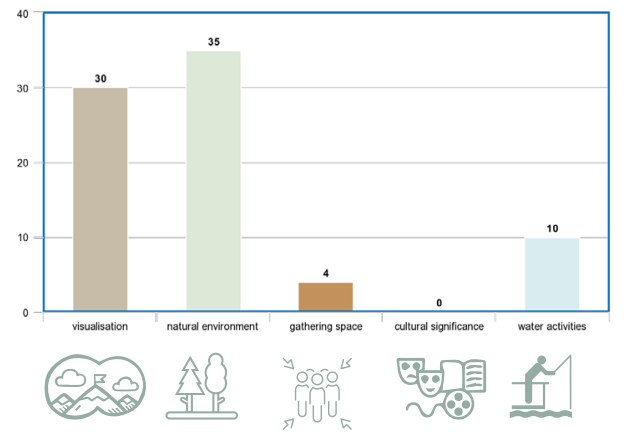

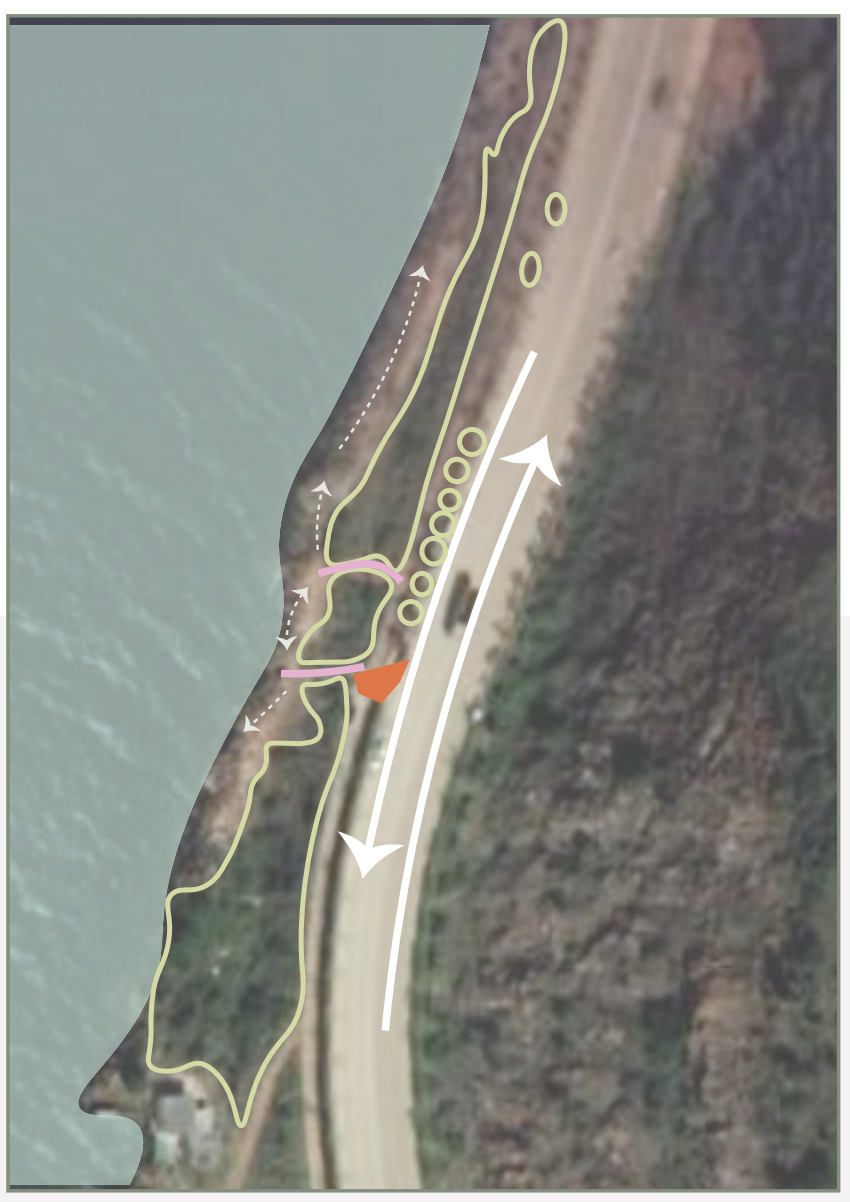

SITE 2.1

This site does provide access to the water, but it is not easy to find the entrance. Visitors park their cars on the driveway and walk ten minutes to this site. Due to the secret location of the space, there are usually no visitors. 


\title{
- -
}

\author{
Cinnamonum camphora \\ Scirpus validus \\ Cyperus rotundus \\ Beccariophoenix alfredii
}

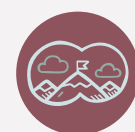

VISUALISATION

People can see the shapes of the mountain from this site.

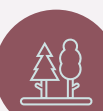

NATURAL

\section{ENVIRONMENT}

The journey from the road to

the water's edge has a beautiful

ecotone, the transition from the forest to a marsh environment

makes this site ecologically

special.(Fig)

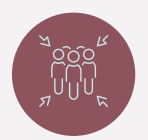

\section{GATHERING SPACE}

The shallow water level can allow children to play with the water.

Overall, it is a beautiful site without any human-made design and

showcases how a natural lakefront should be. There is opportunity to include the exciting ecotone of the site to future designs.

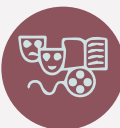

\section{CULTURE SIGNIFICANCE}

There is no existing culture, or any history people can find about the site.
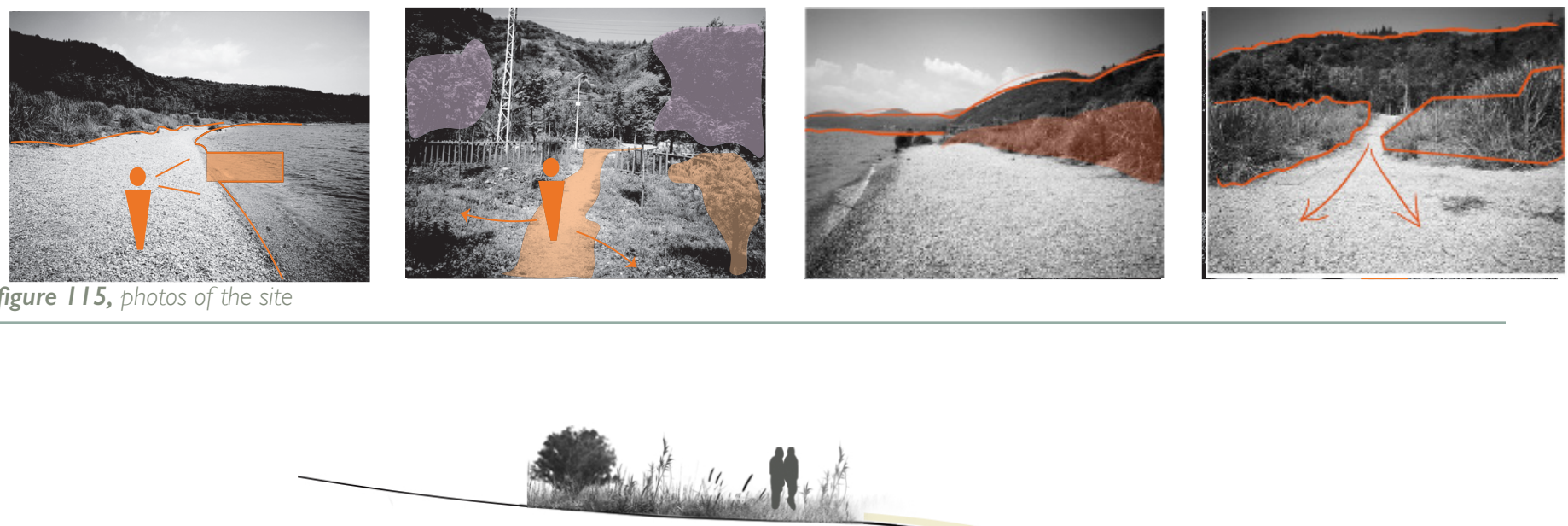


\section{SITE 2.2}
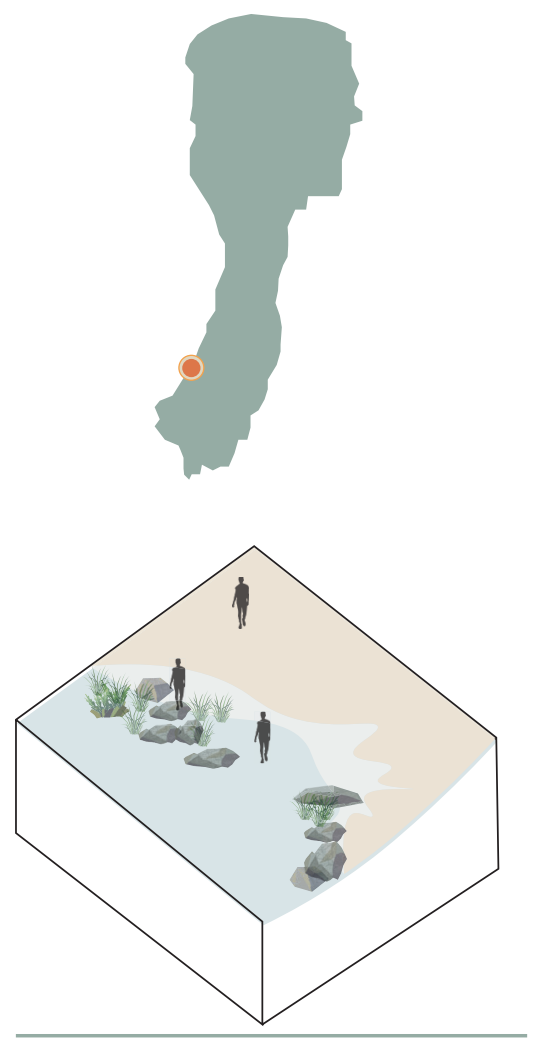

\section{DATA ANALYSIS}

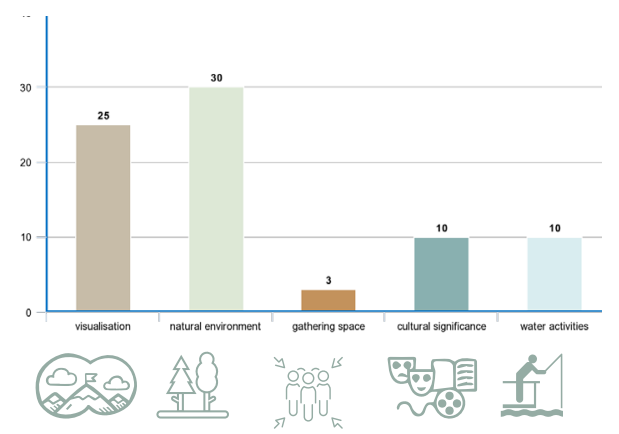

from top to bottom.--figure I I 7 II / I I9

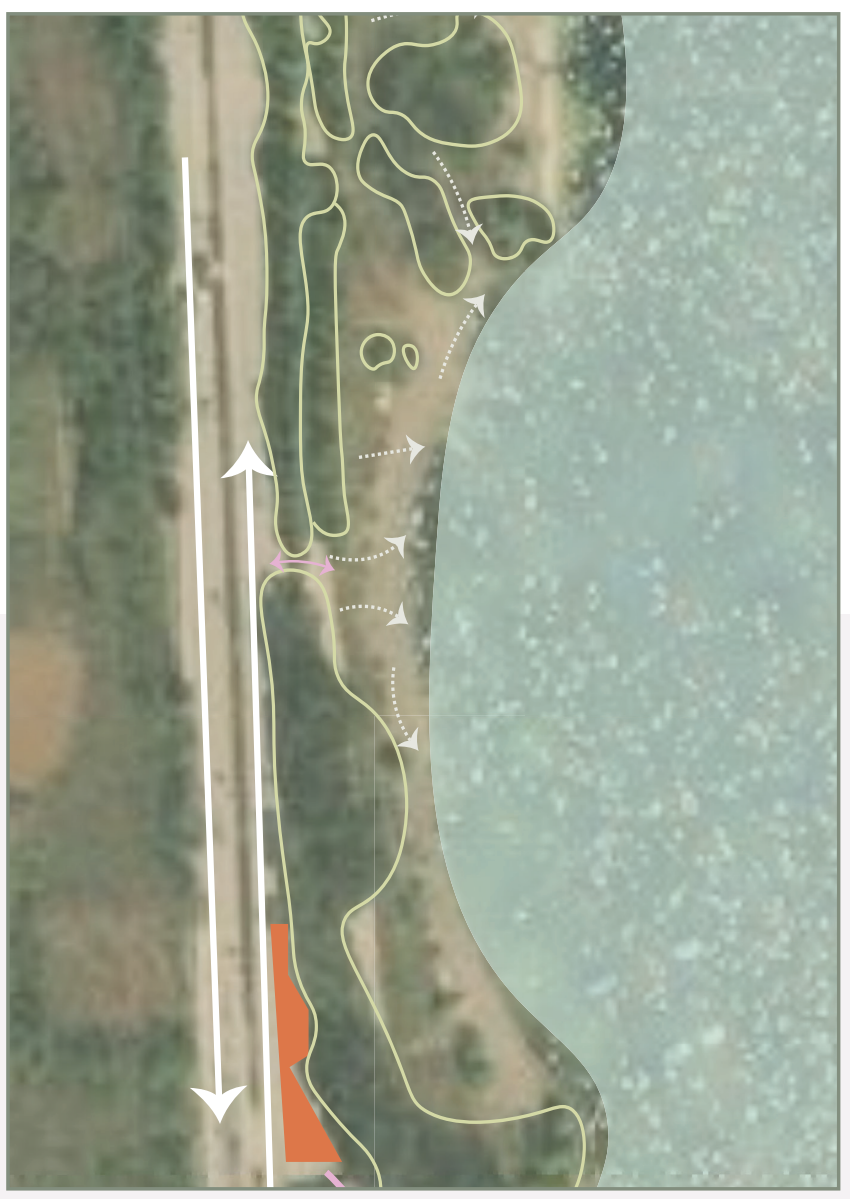

SITE 2.2
Similar to Site 2.I, Site 2.2 is hard to find and inconvenient to park a car. Due to the lakefront's limited size, the site is not flexible to develop any changes. 


\section{- - nums}

Medicago linn

Beccariophoenix alfredii

Scirpus validus

Cyperus rotundus

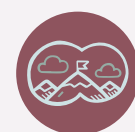

VISUALISATION

The trees blocked the view of the pathway, concealing any

view of the lake, until visitors are proximately on the water's edge. People have a beautiful mountain view from this site.

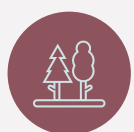

NATURAL ENVIRONMENT

The journey, from the road to the waters edges has a beautiful ecotone. Some of the trees are I.5 meters tall, which blocked the view of the lake.

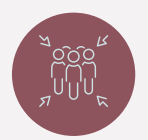

\section{GATHERING SPACE}

There are no visitors using the site because the entrance is hard to find, and the access is not attractive.

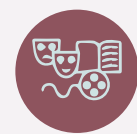

\section{CULTURE SIGNIFICANCE}

One can find an abandoned canoe left on the lakefront, which makes the space appear to have symbolic references.
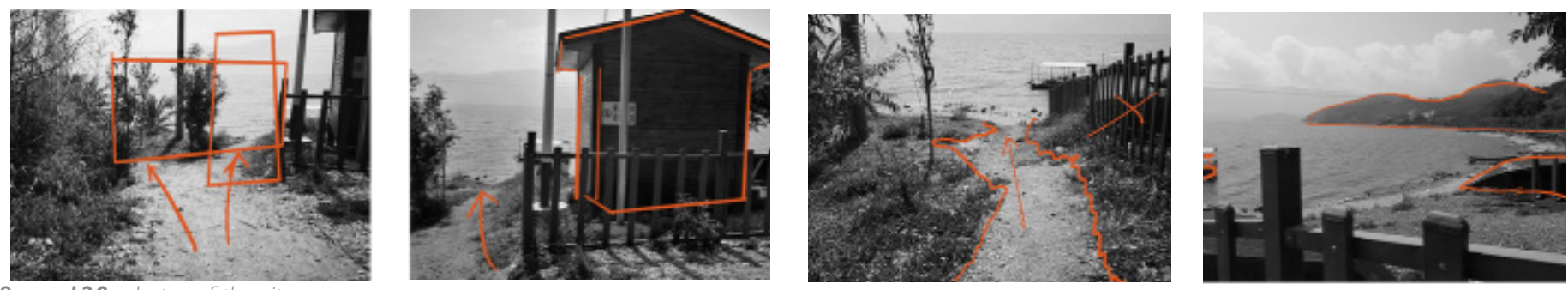


\section{SITE 2.3}
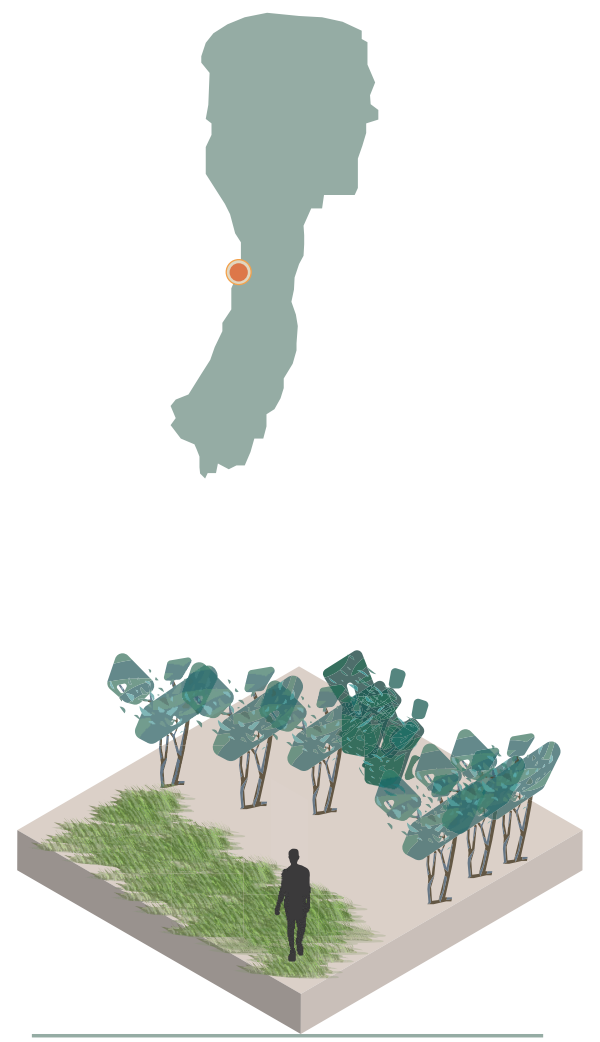

\section{DATA ANALYSIS}

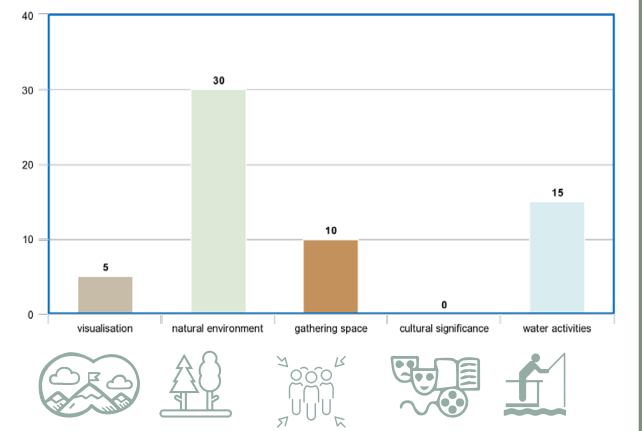

from top to bottom.--figure I 22 I 23 I 24

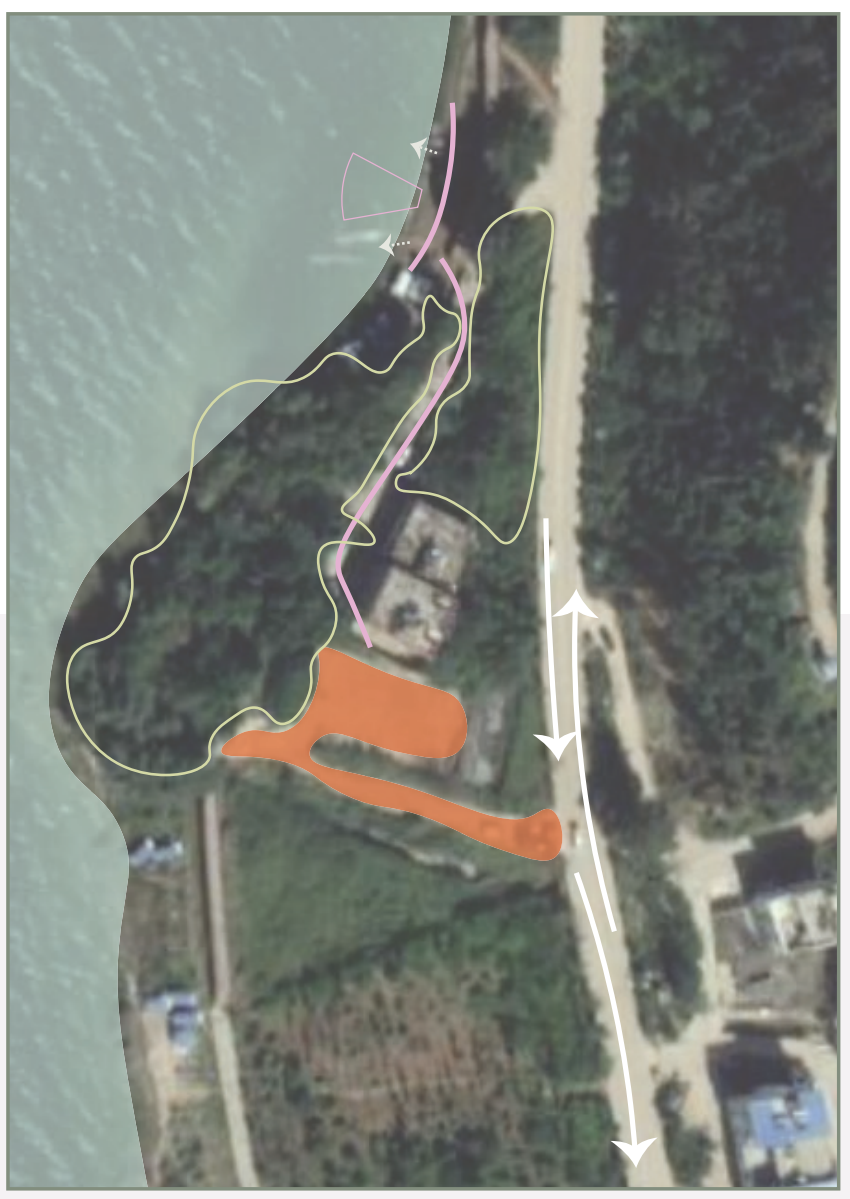

SITE 2.3
It has a carpark around the site, and it makes many people come to use this site. Also, because of the tree canopy and grassy footings, there is a relaxing atmosphere, as shade and lake views are combined. This invites people to sit and stay longer. 


\section{-}

\section{PLANTS}

Cinnamonum camphora

Salix babylonica

Cyperus rotundus

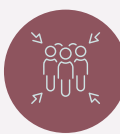

\section{GATHERING SPACE}

The low water level allows people to engage in water activities on the site.

However, due to and and soil sediments, the water in this site is not transparently clear and unappealing.

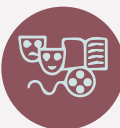

\section{CULTURE SIGNIFICANCE}

There is no existing culture, or any history people can find, about the site. time, the lake scene sharply appears to people when travelling through the bush. site are about 2-3 meters high, and the trees form a canopy. There are a few tussocks growing on the lawn.
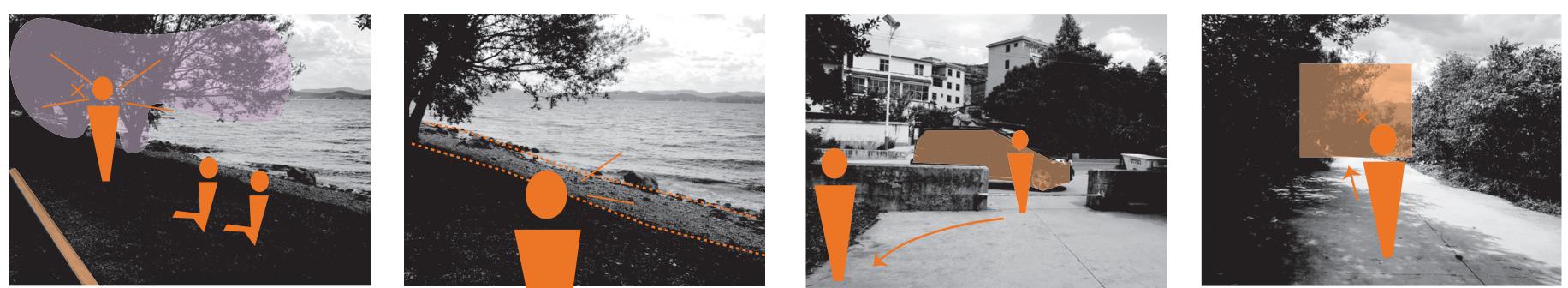

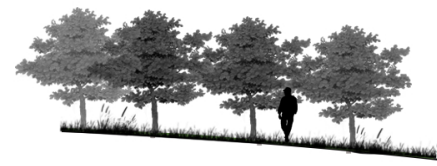




\section{SITE 2.4}
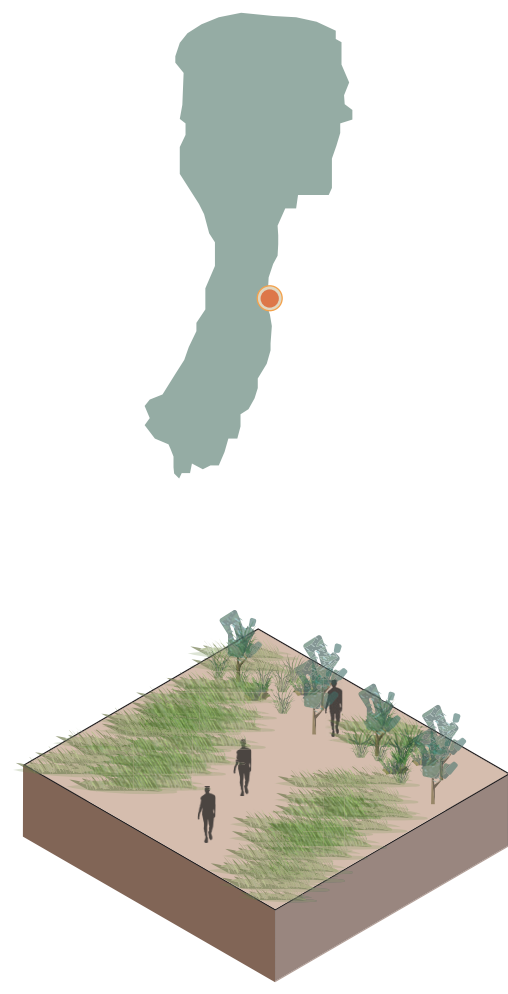

\section{DATA ANALYSIS}

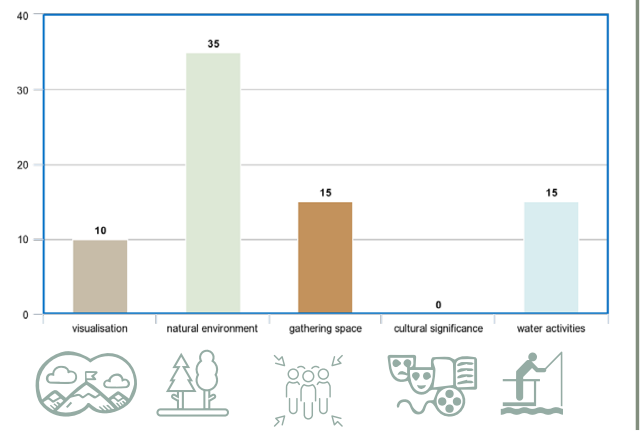

from top to bottom. --figure / 26 /27 / 28

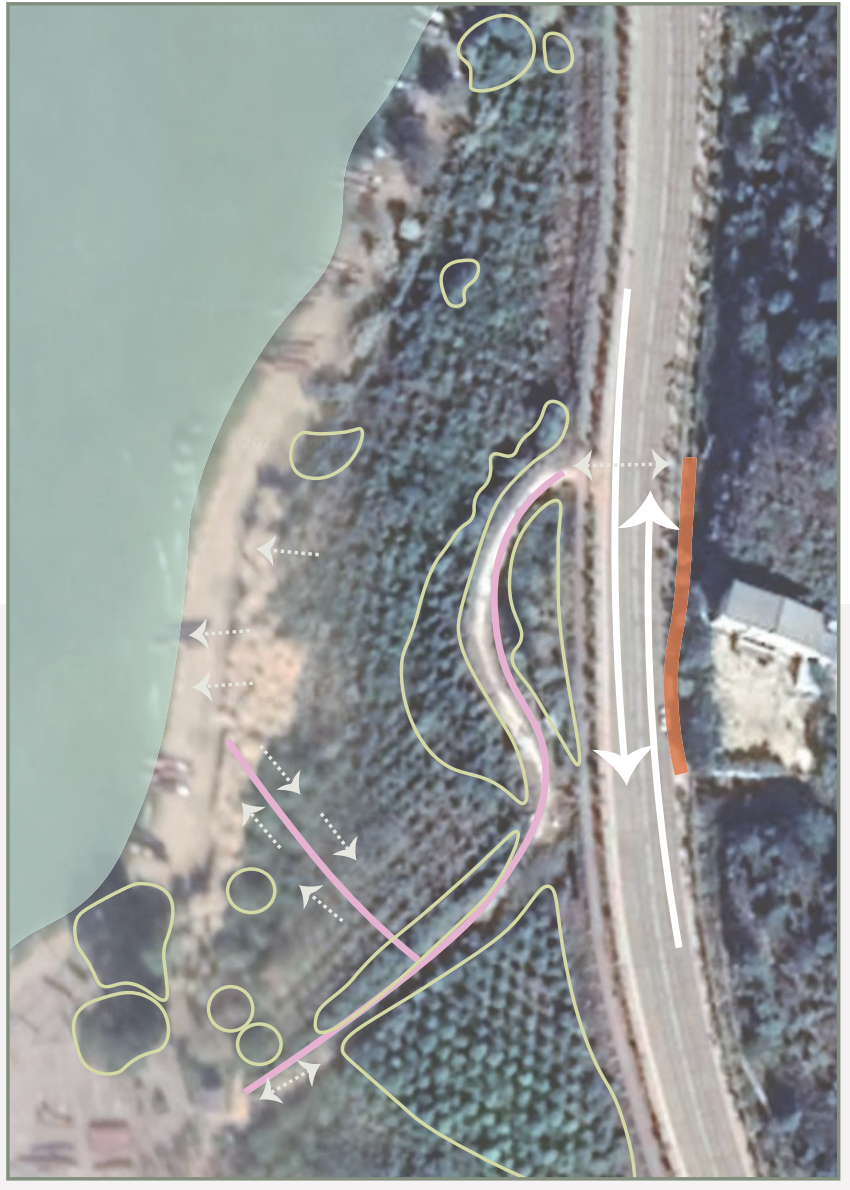

SITE 2.4
Compared with the other three sites with a natural environment, Site 2.4 is the location with the highest density of plants. Even though it is not easy to find, some people come to the site to use it as a picnic area. If people want to park their car and walk to the site, they have to cross the road without any pedestrian aided assistance. 


\section{-}

\section{PLANTS}

Cinnamonum camphora

Salix babylonica

Cyperus rotundus

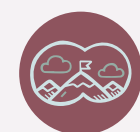

VISUALISATION

It has a close view of the

lakeshore ecotone. However, some individuals will damage the vegetation by driving closer to the water's edge.

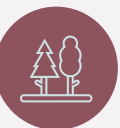

NATURAL ENVIRONMENT

It has diverse types of plants that include trees, shrubs, and grass.

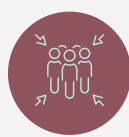

\section{GATHERING} SPACE

There is no carpark around the area, so many visitors drive their motorcycle to this site.

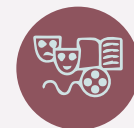

\section{CULTURE SIGNIFICANCE}

There is no existing culture, or any history people can find about the site.
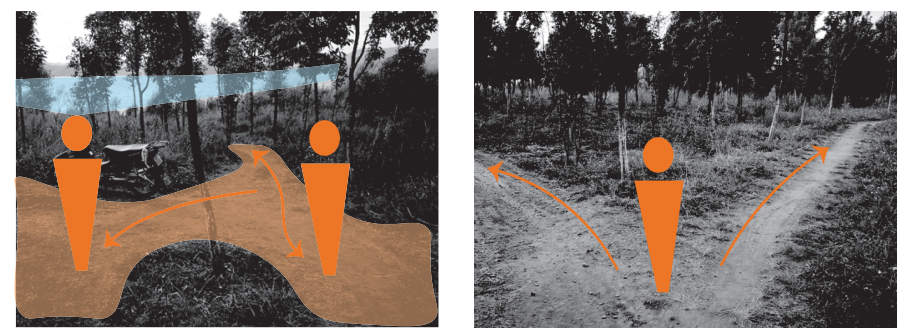
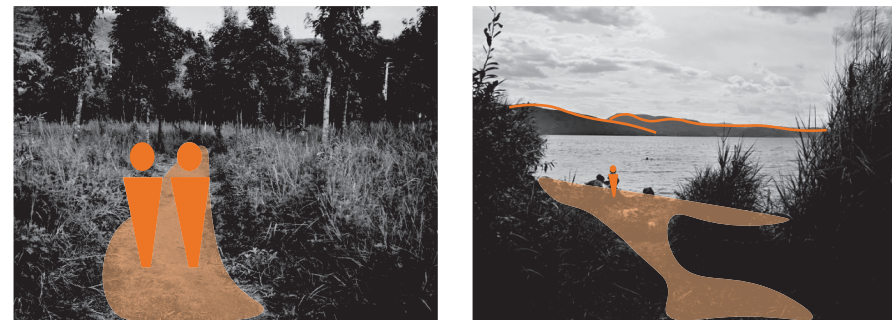


\section{SITE 2.5}
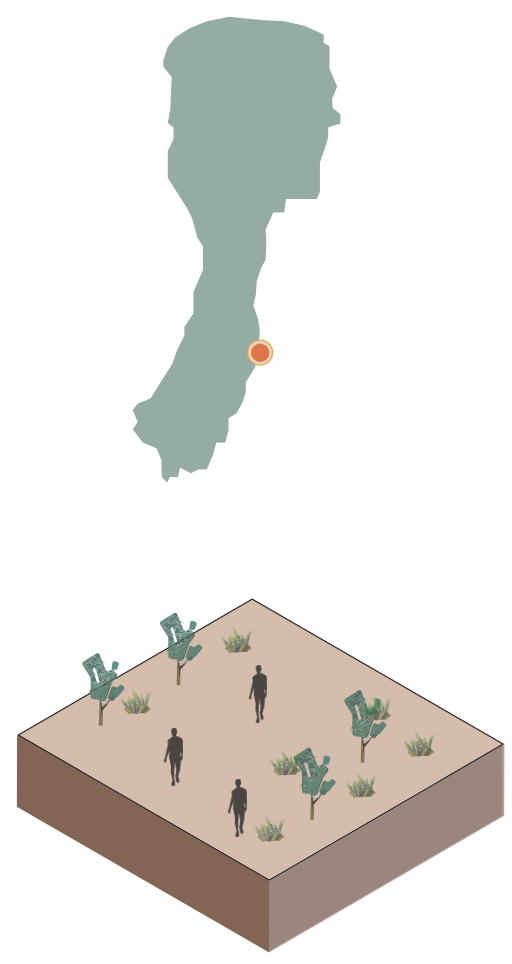

\section{DATA ANALYSIS}

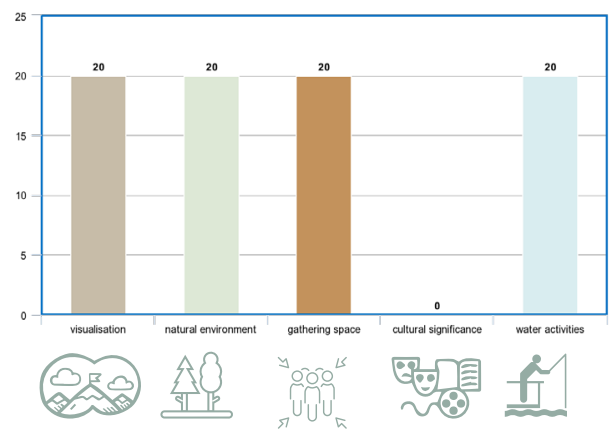

from top to bottom.--figure / 3 I I 32 I 33

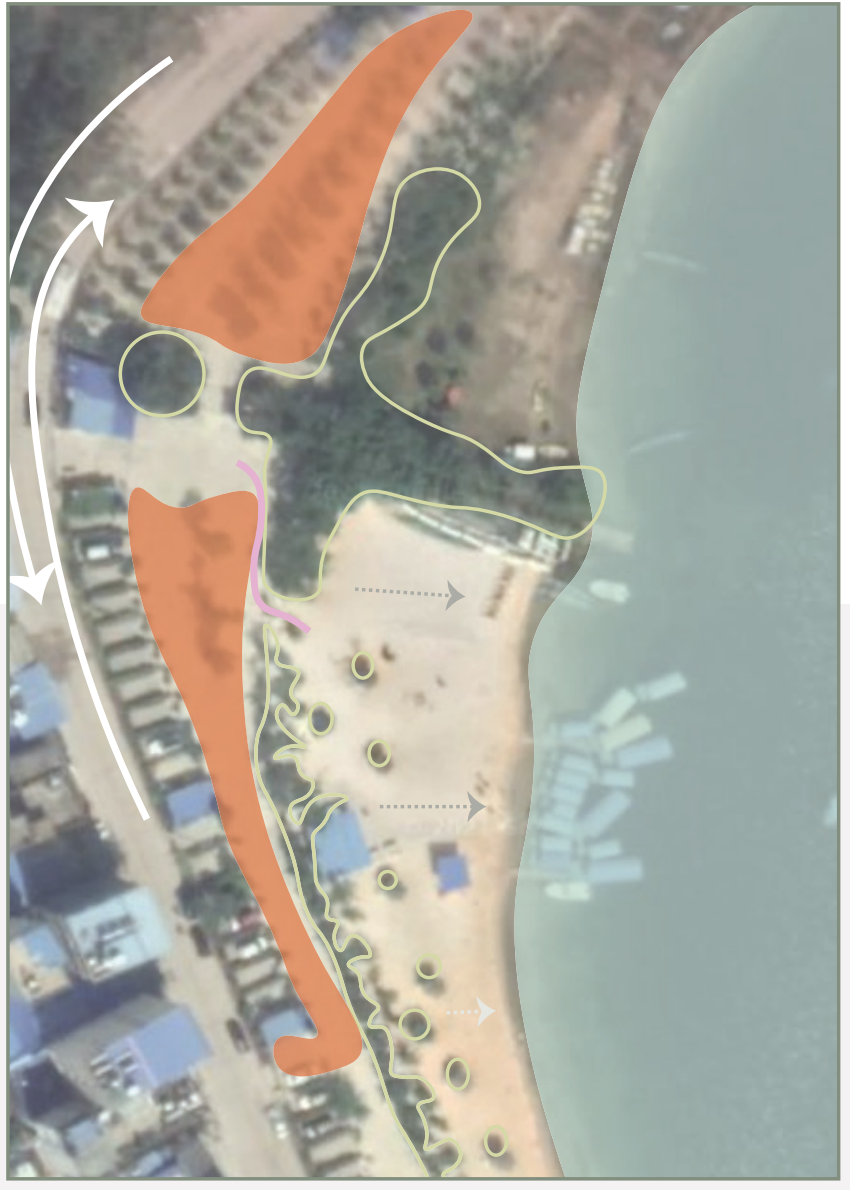

SITE 2.5

This site has a public park that allows people to take a boat to explore the area. The design of the park is simple including vegetation which is limited to a few trees at the water's edge. 


\section{- - nom}

Cinnamonum camphora

Salix babylonica

Cyperus rotundus

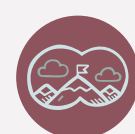

\section{VISUALISATION}

The sediments on this site are combined with gravel and sand, so the texture of the ground is friendly to people who want to play with water. However, during a rainy day, the transparency of water might will be low.

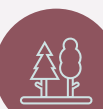

NATURAL

\section{ENVIRONMENT}

There are many canopy trees on the water's edge, attracting people to sit beneath them
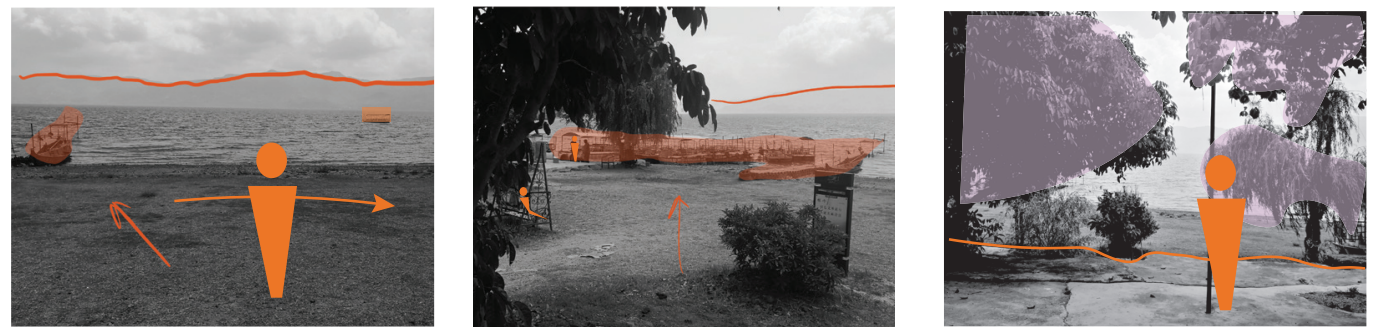

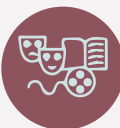

\section{CULTURE SIGNIFICANCE}

There is no existing culture, or any history people can find about the site.
Due to the convenient location, there is a high visitor flow. However, there are no seats on this site, which makes the visitors only stay briefly.
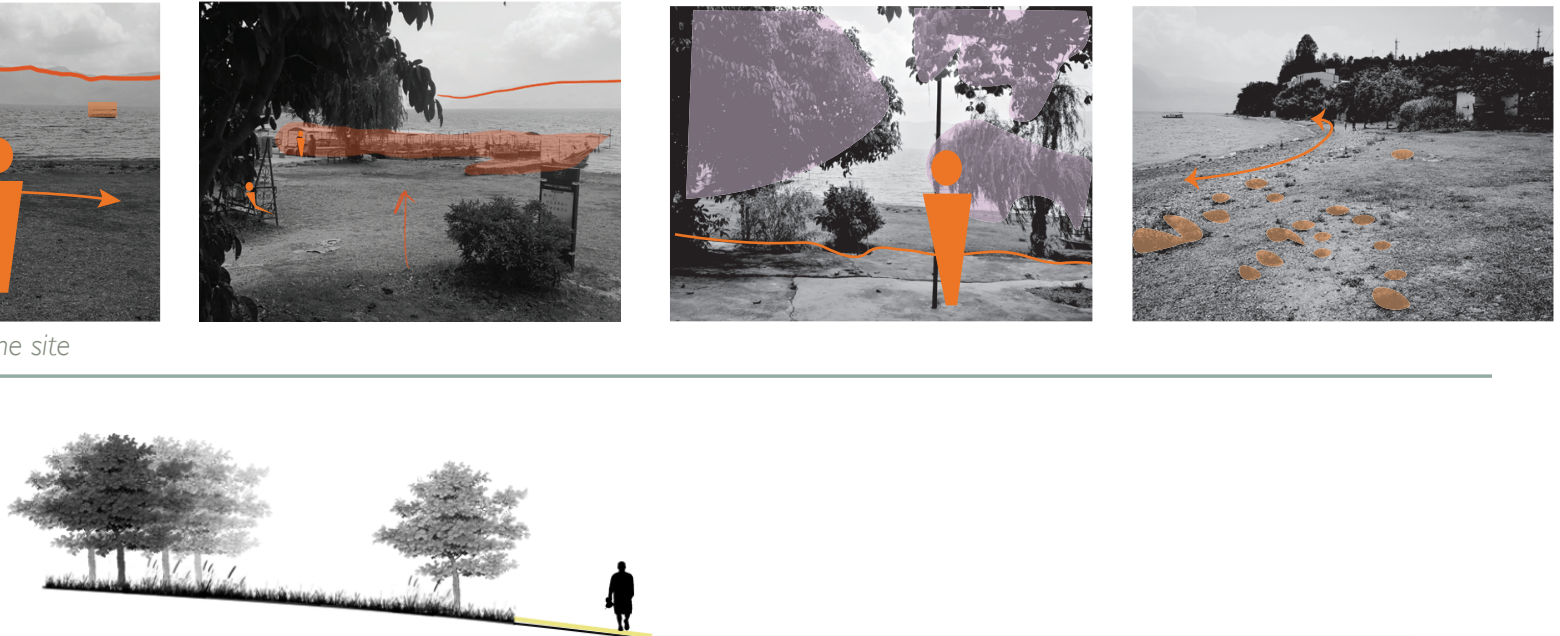


\section{SITES WITH CULTRUAL SIGNIFICANCE}

\section{Site Values:}

\section{visualisation:}

has mountain view without any trees hide the views 30

has mountain view but hidden by trees 20

has open water views 15

has lake views but hidden by trees 10

has lake views but most area has been hidden 5

natural environment:

massive planting 25-30 (according to the density of plants)

only a few trees 5- 10

has ecotone 10

gathering space:

has different types of people use it 15

has most of the people use it 20

has few people use it 5

only young people use the space 5

cultural significance:

has mountain views 10

has a little bit culture remains 10

Used to settle by Dian history 25

has fossils 30

With Dian legend 30

\section{water activities:}

can see the lake with long-distance 5

can see the lake closely 10

can touch the water 20

has different types of water activities 10

can take a boat 5

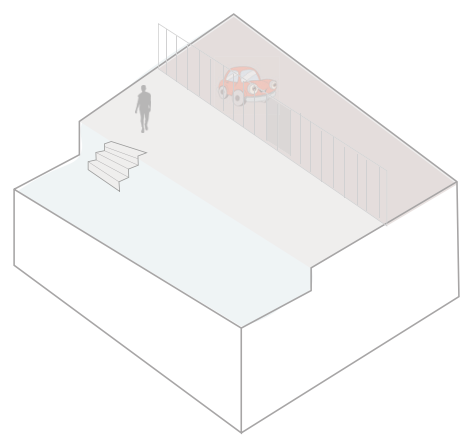

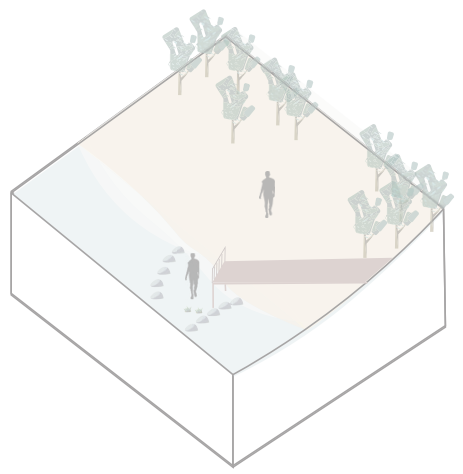

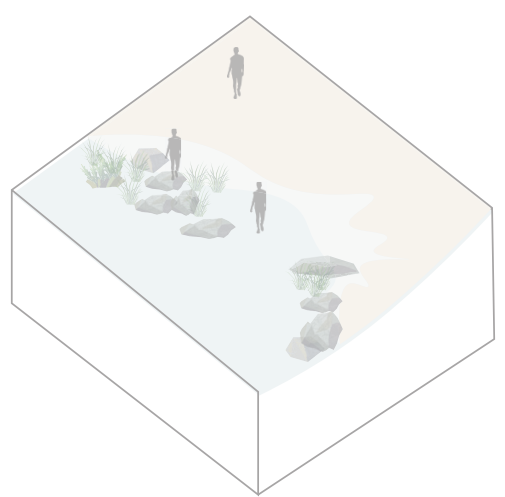




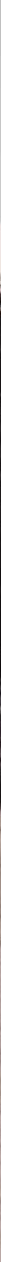

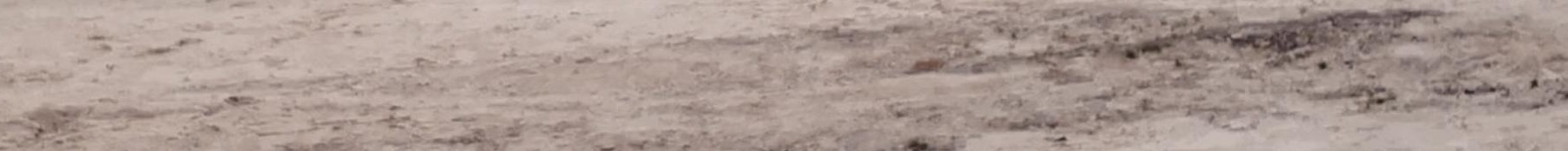

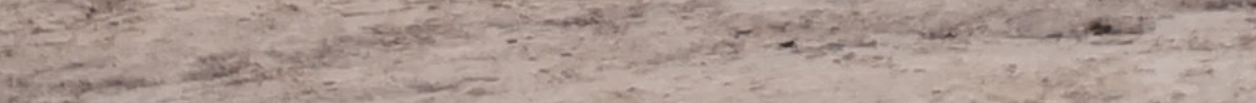

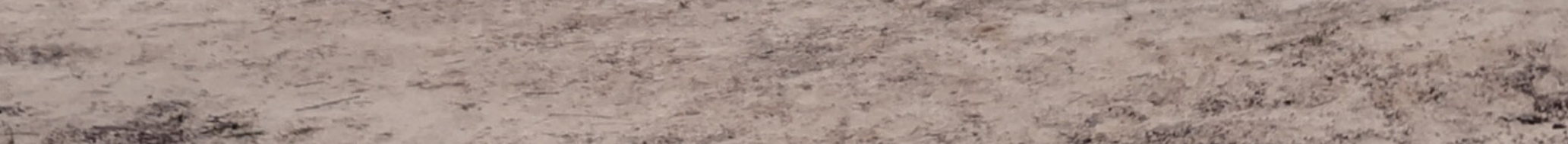

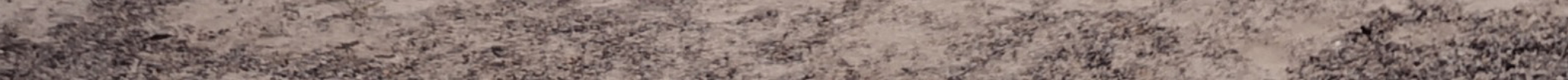
H.

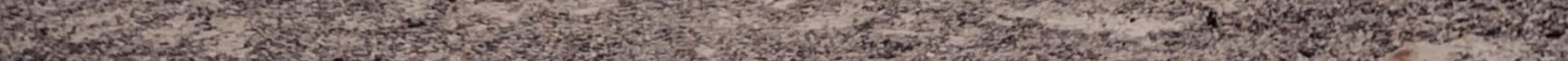

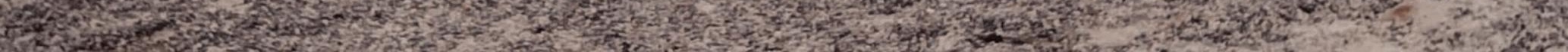

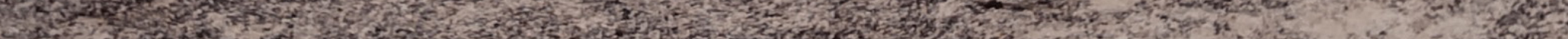

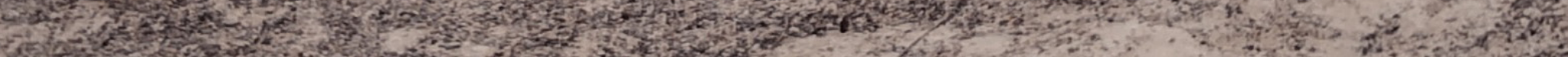

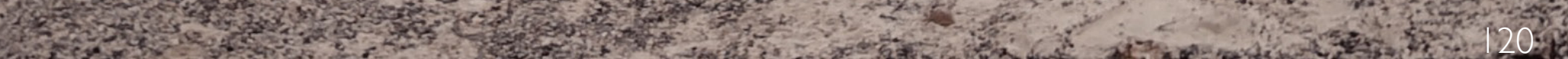

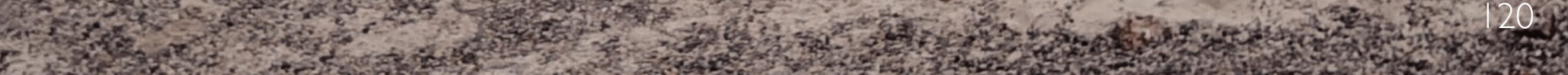
E 


\section{SITE 3.I}
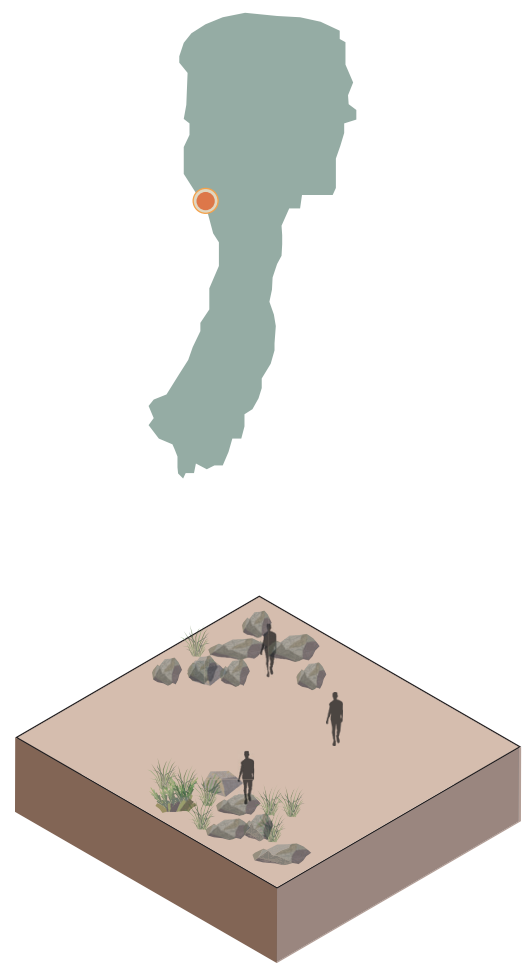

\section{DATA ANALYSIS}

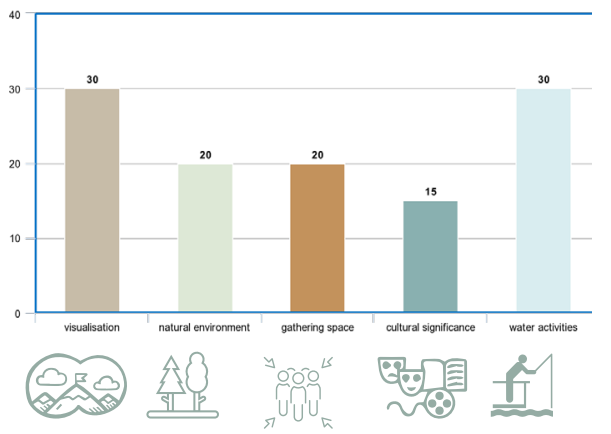

from top to bottom.--figure / 36 I37 I38

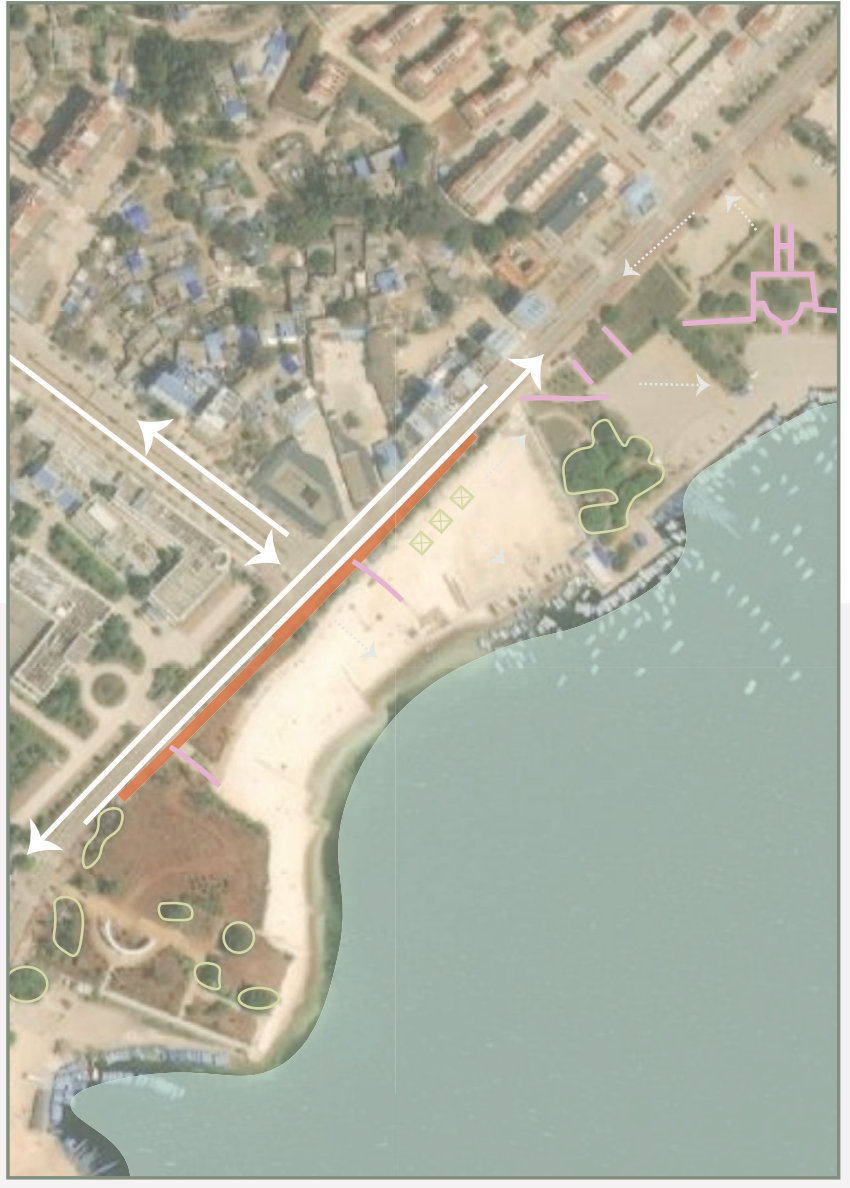

SITE 3.1

The public beach is just next to a carpark, and visitors can enjoy taking a boat on this site The location is close to a legend where a fish king is supposedly living. To make the site look like it has Chinese features, the designer utilised Chinese Architecture when creating the canopy. 


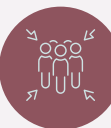

\section{GATHERING SPACE}

On this site people can use a boat from the beach and enjoy other water activities.

the boat or canoe on this site. However, the views on this site are not very open compared to other sites. Because of the tall trees surround the park, people can only see what's happening within the site.

NATURAL

\section{ENVIRONMENT}

The palm trees

(Beccariophoenix alfredii)

give the site a tropical

atmosphere.

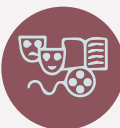

CULTURE SIGNIFICANCE

Except for the Chinese styled canopy, there are no cultural features on this site. The canopy is similar to any other canopy pavilion in China.
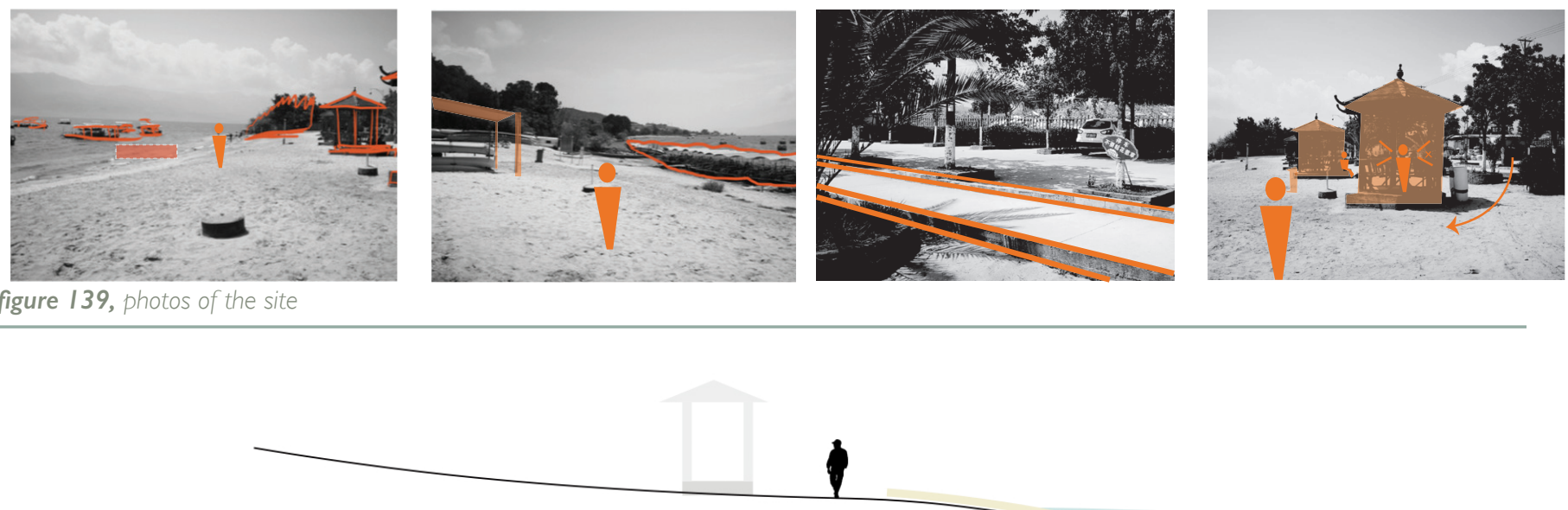


\section{SITE 3.2}
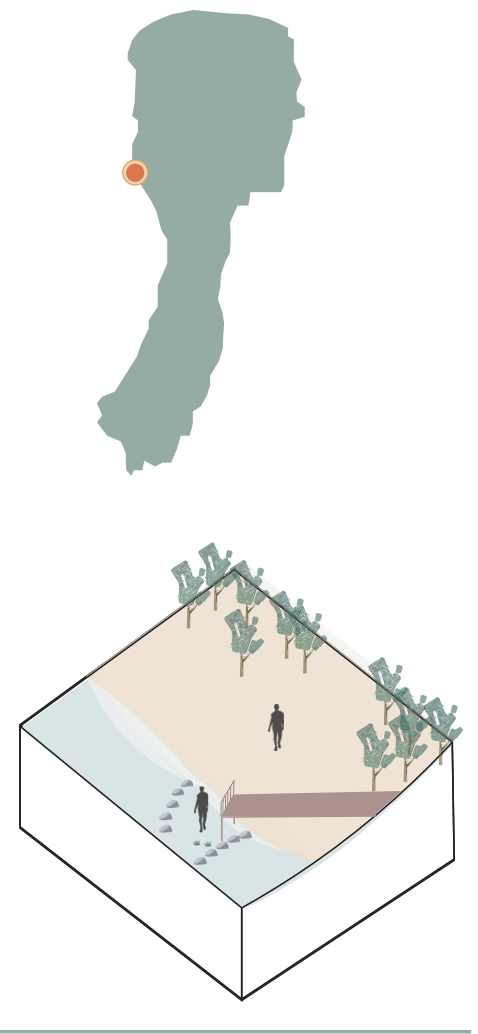

\section{DATA ANALYSIS}

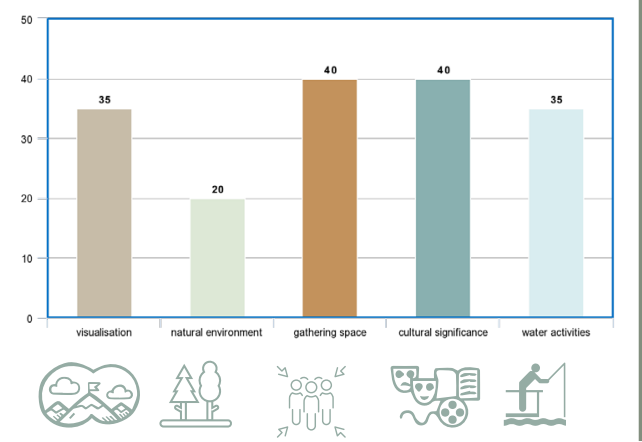

from top to bottom.--figure / 4 I I 42 I 43

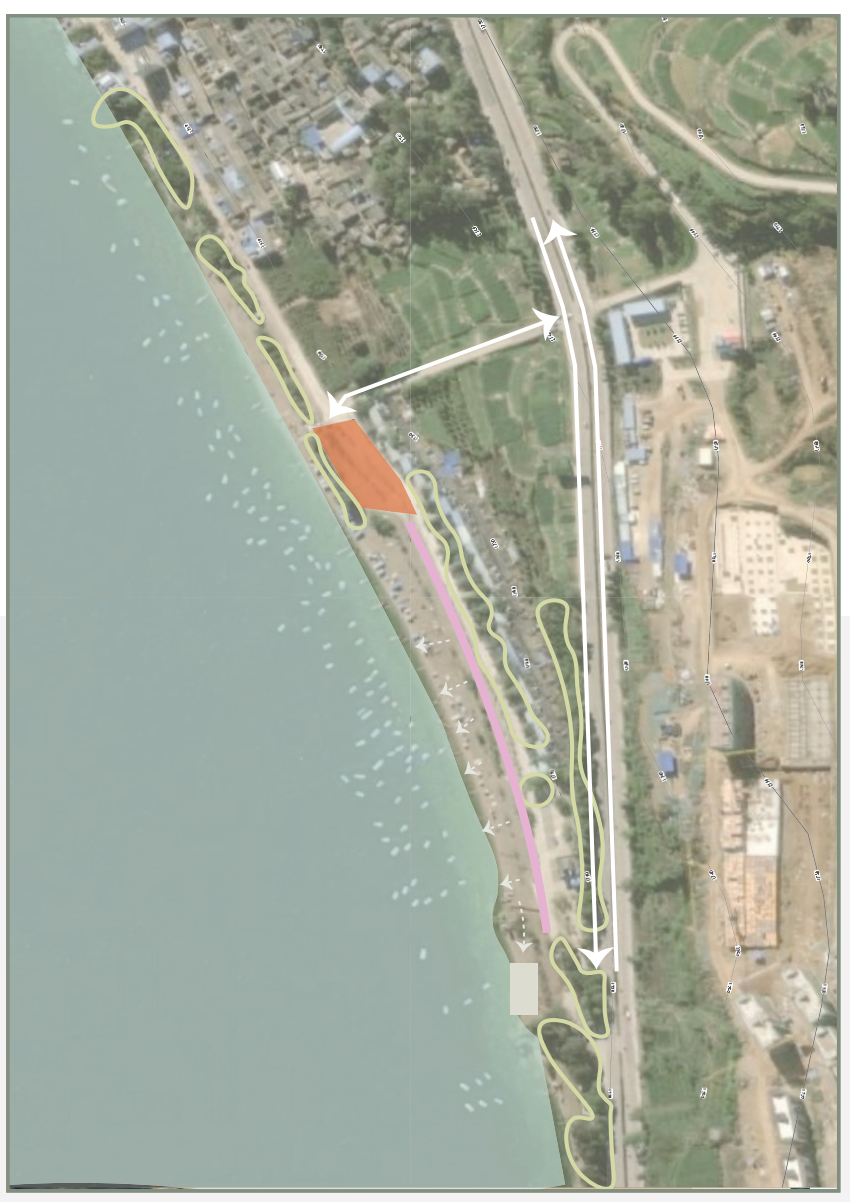

SITE 3.2
Only this public park has some feature of Dian culture around Lake Fuxian. The location has the highest visitor flow, as many visitors come and use the site. There are many opportunities to improve the Dian cultural features on this site. For example, the park has a sculpture illustrates the tool, for improving the Dian culture, the site can allow people experience how Dian people used to fish. 


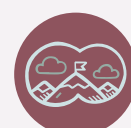

\section{VISUALISATION}

The primary materials of this site are sand and concrete. The mountain view is excellent; it can be defined as the best mountain view around the entire lake.

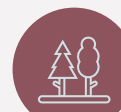

NATURAL ENVIRONMENT

The site includes few trees that can be utilised as a natural sheltering space.

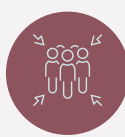

\section{GATHERING SPACE}

The site includes a beach, lookout, and sheltering space that allows people to do different activities such as beach volleyball, lying down and play with water.

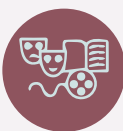

\section{CULTURE SIGNIFICANCE}

The entrance from the carpark has a structure that shows an ancient tool Dian people used for fishing. It is engaging and informative to the visitors as it provides the history of the site.
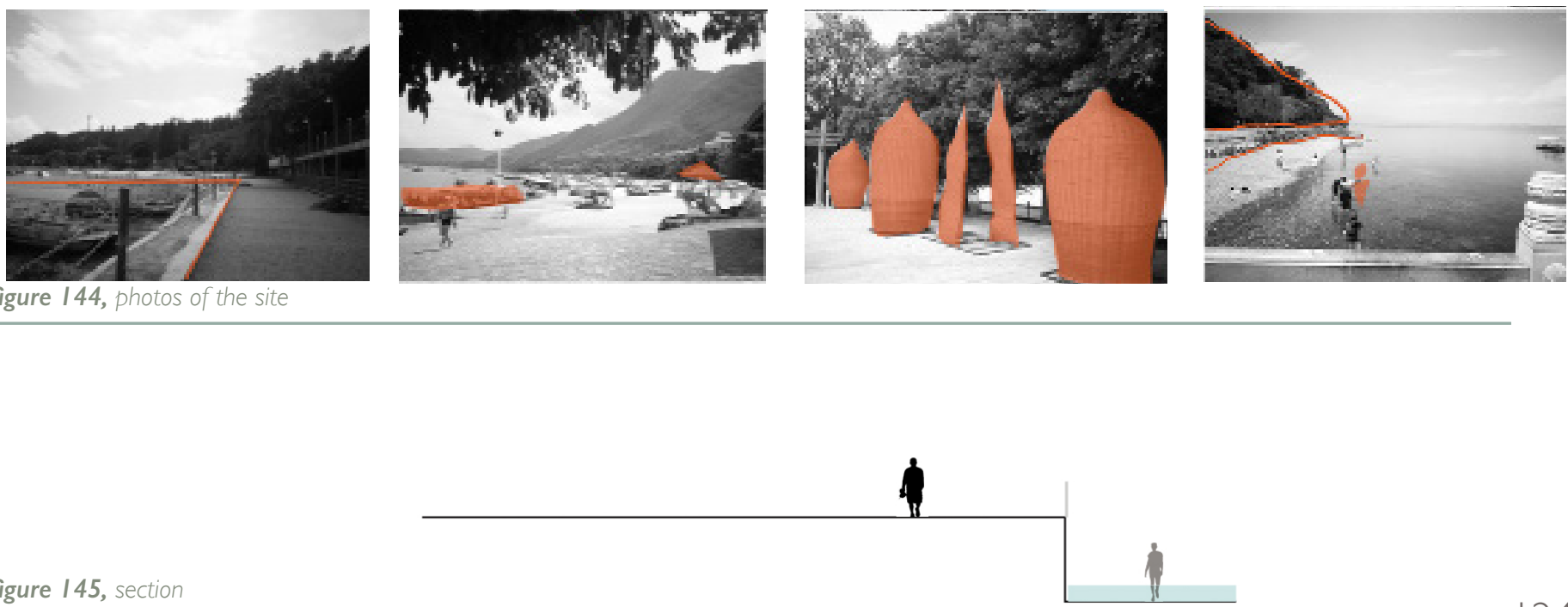


\section{SITE 3.3}

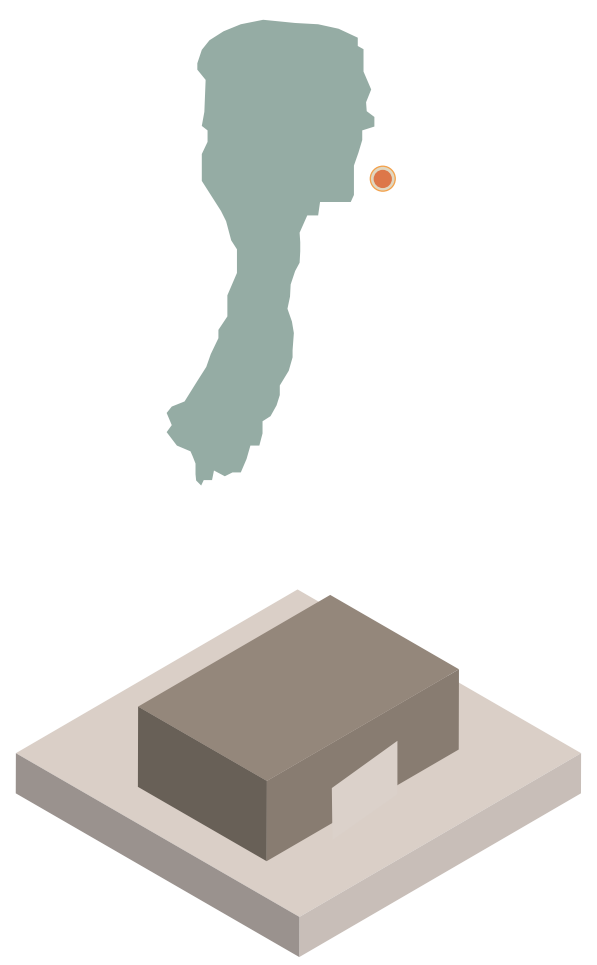

DATA ANALYSIS

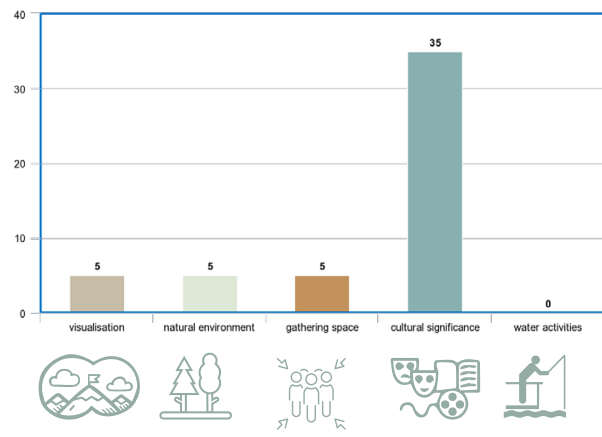

from top to bottom.--figure/46 I47 I48

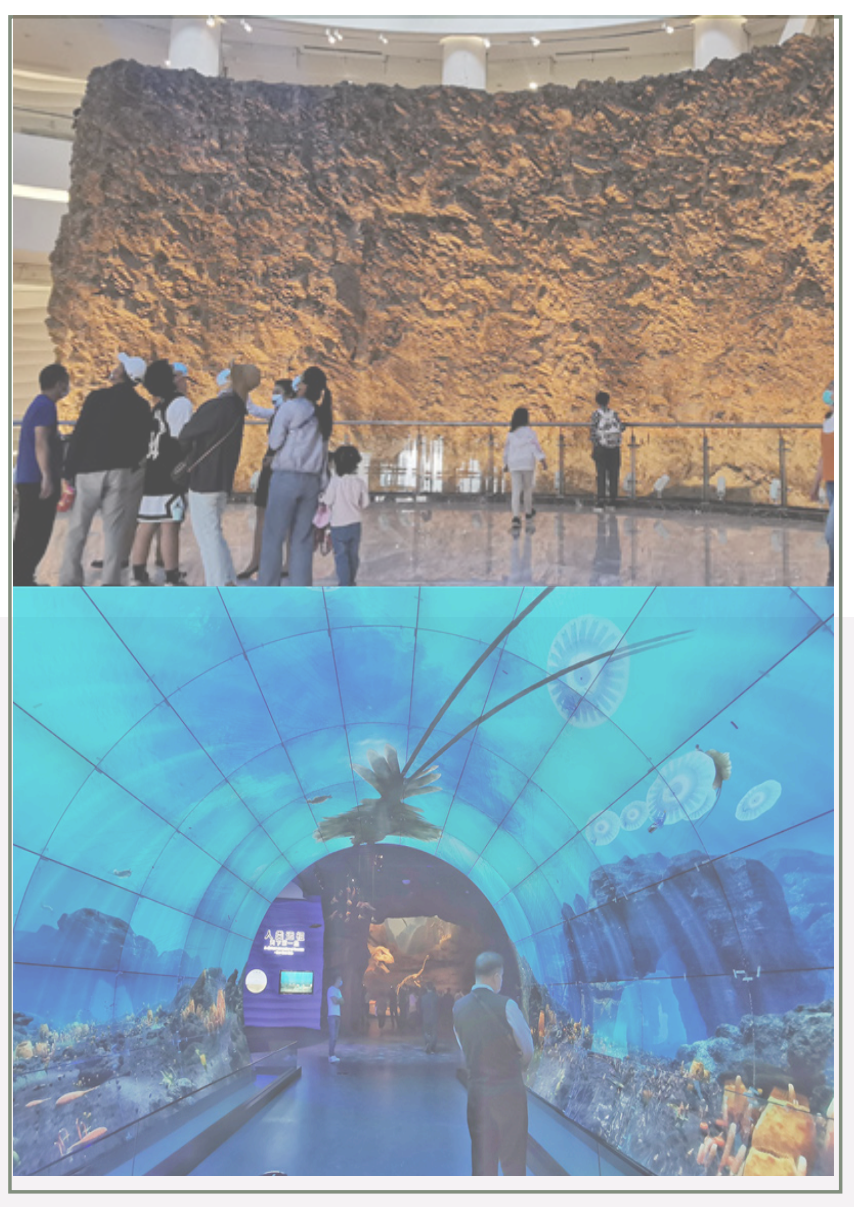

SITE 3.3

As a celebreation of the site, this museum was created in 1992 to educate visitors on the paleontology of the site, from 500 million years ago until present day. 


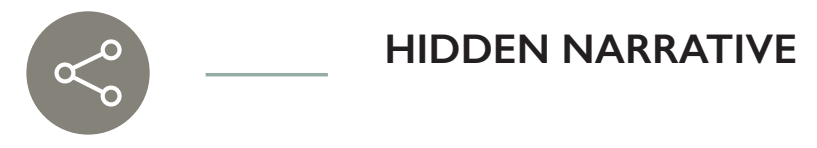

Museum to show tha paleontology during Cambrian Period in Lake Fuxian

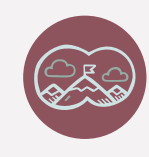

VISUALISATION

The site does not have any lake views or mountain views.

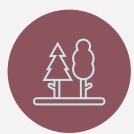

NATURAL ENVIRONMENT

It only has a few trees planted within the carpark space.

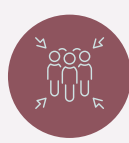

\section{GATHERING SPACE}

The site is inconvenient for people to travel to because it is a 30 -minute drive from the nearest lakefront. The driveway needs to be developed because it is bumpy and rough. Also, probably the museum is not attractive to let people spend 30 minutes driving to come the site.

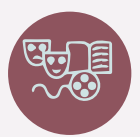

\section{CULTURE SIGNIFICANCE}

The Cambrian era fossils found in this site is one of the key features of the site. This site is the fossil founding site, which is special for the lake and the city.

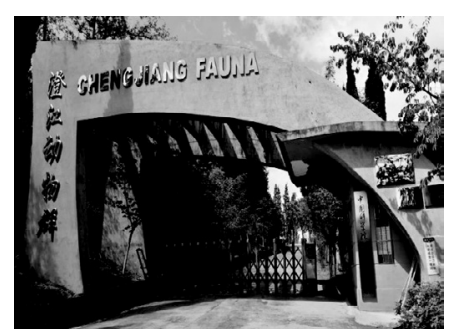

figure 149, photos of the site
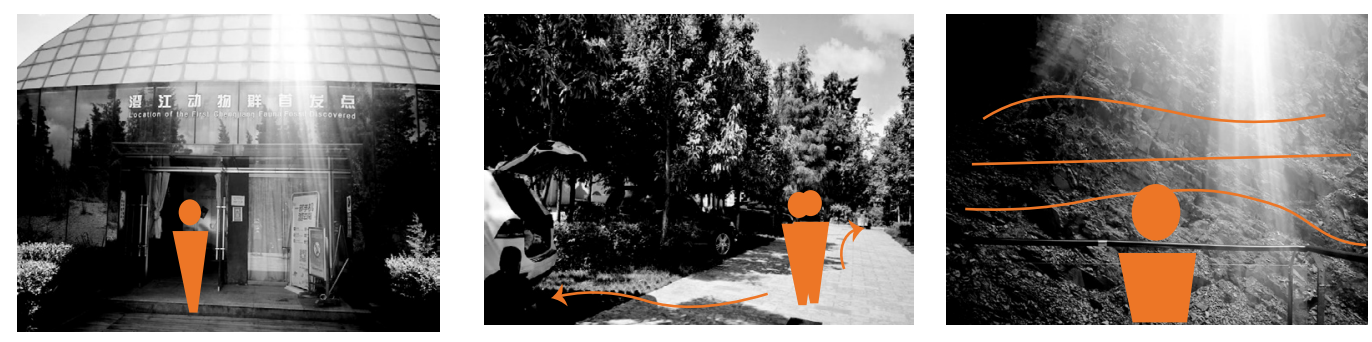


\section{SITE 3.4}

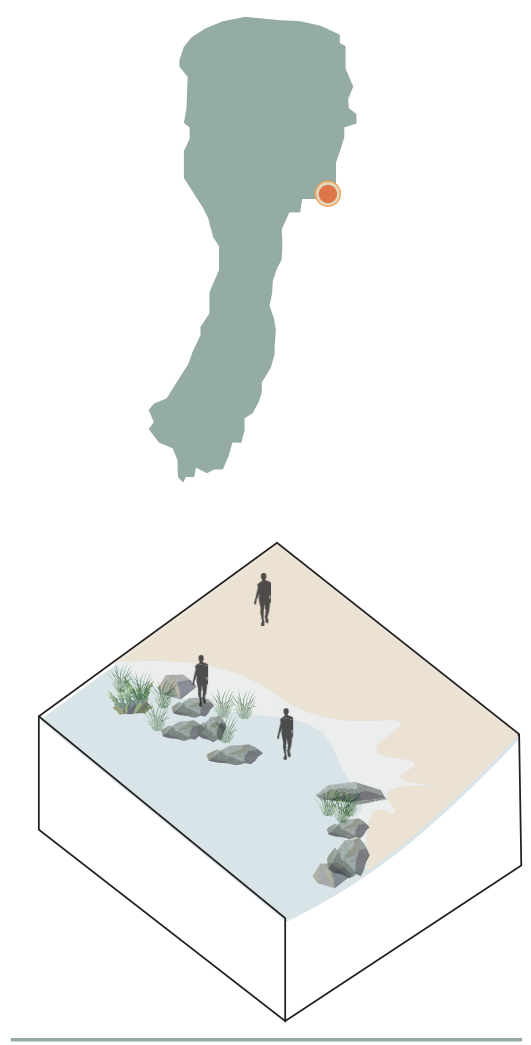

\section{DATA ANALYSIS}

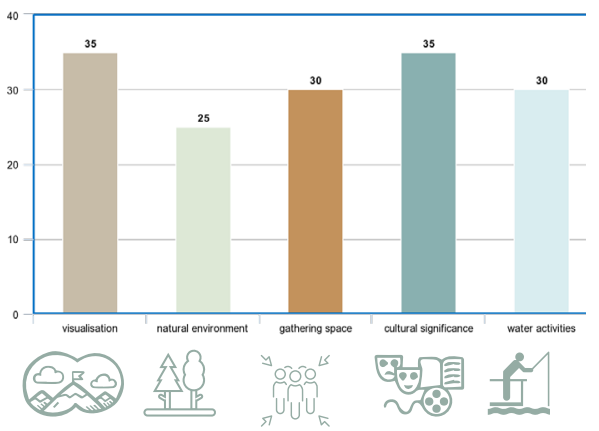

from top to bottom.--figure I5I I52 I53

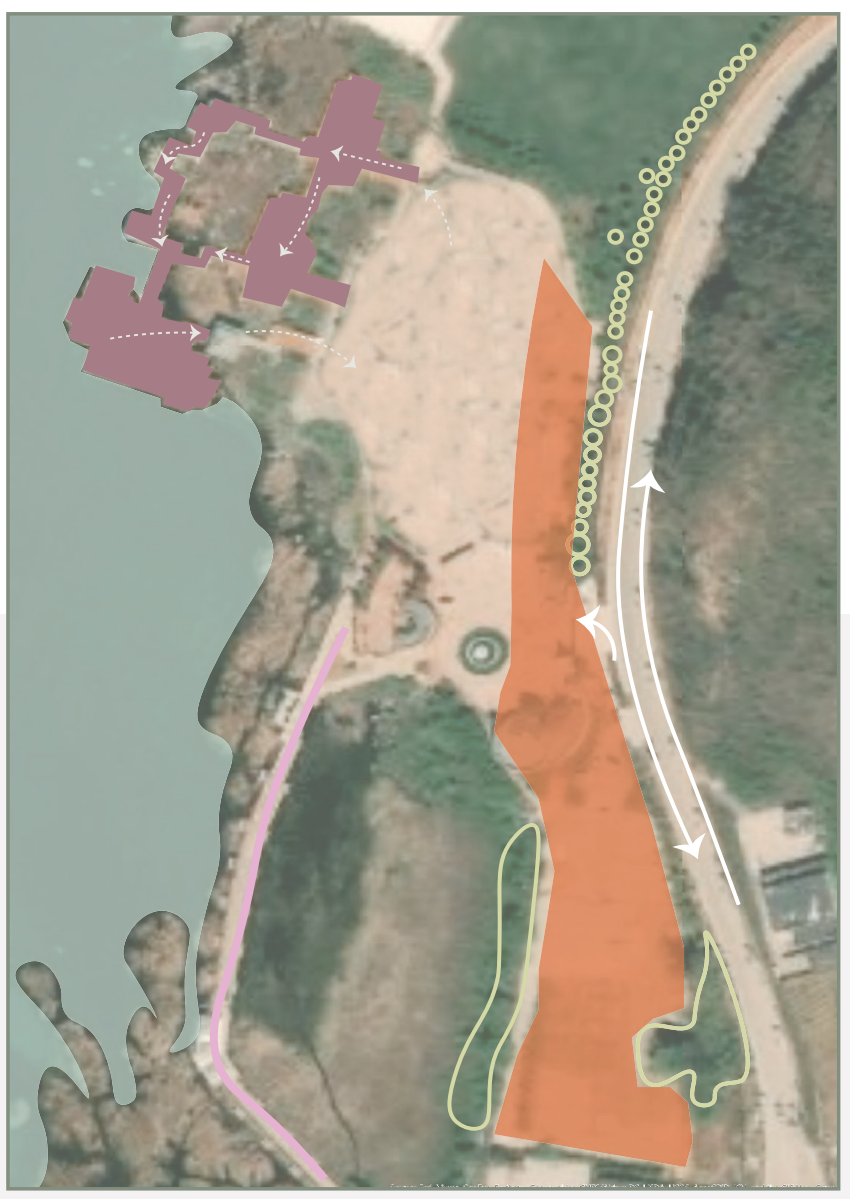

SITE 3.4
This site is uniquely close to the underwater building remained from the Dian period. People are not allowed to dive or swim in the lake unless they have a freshwater diving certificate. 
details of underwater structure will expand more

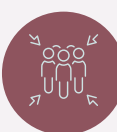

\section{GATHERING SPACE}

\section{The waterfront part is unique because it has big stones on the water's edge. People can enjoy standing and walking on the stones in the water \\ Few wetland plants are growing \\ on the site. Also, there are plants growing in the gaps of the stones. It has formed unique pattern on the water} the pattern is quite similar to the man-made pattern on the underwater remains.

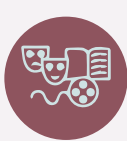

\section{CULTURE SIGNIFICANCE}

The walkway has some features of Chinese Architecture that create a sense of place. The Chinese features are on the shape of the sheltering pavilion. However, the pavilion can be seen in other places in China, and it does not highlight the identity of this site.
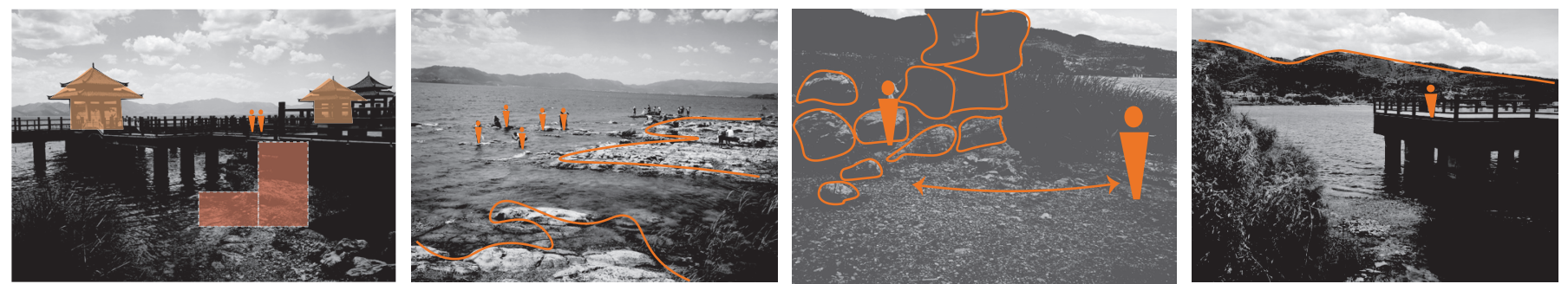

figure 154, photos of the site

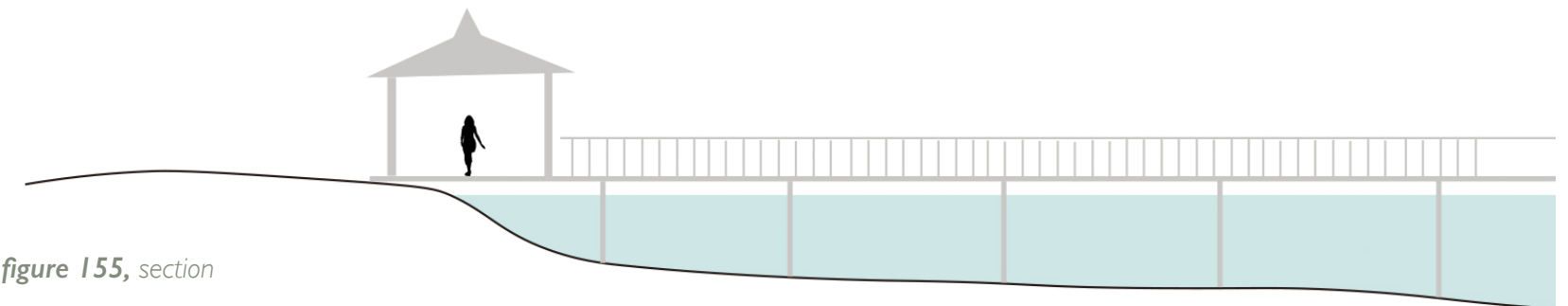




\section{SITE 3.5}

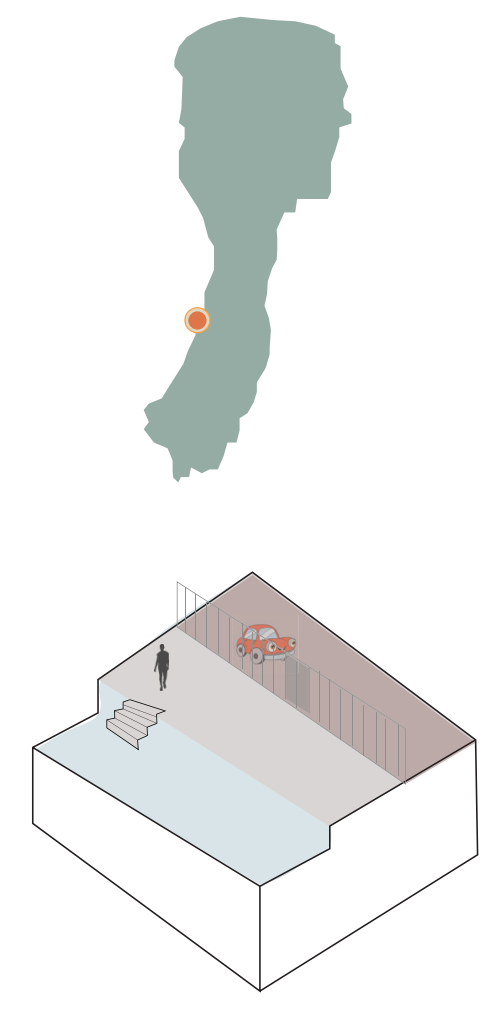

\section{DATA ANALYSIS}

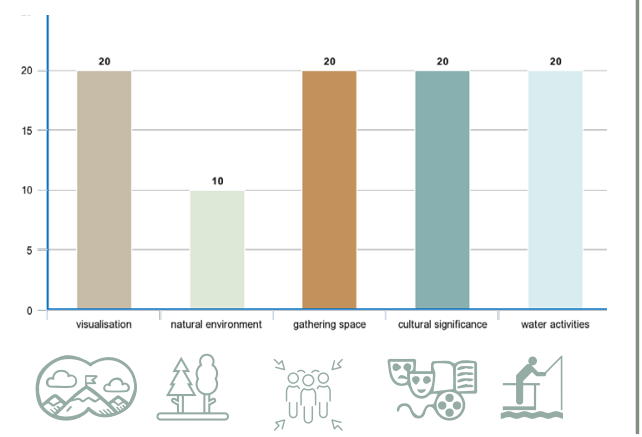

from top to bottom.--figure I56 I57 I58

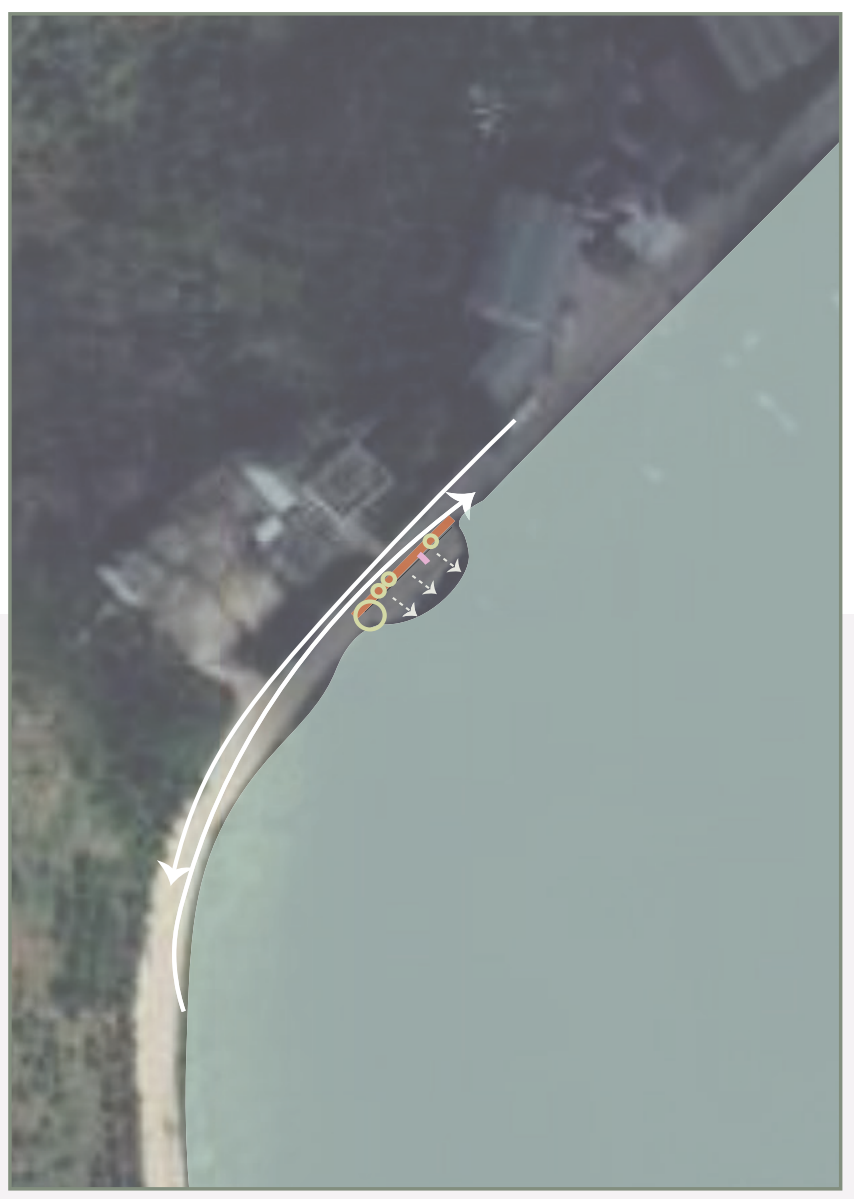

SITE 3.5
Unlike the other four sites (Sites 3.1 , 3.2, 3.3, 3.4), this site does not contain any vital cultural significance. This site used as a fishing site for 20 years, however, due to over fishing the number of native fish has decreased. To protect the fish, the government has not allowed people to go fishing in the lake since 2015 (Yunnanwang, 20I5), making the site less popular than before the law change. This site only has three carpark spaces, and is often full through out the day. 


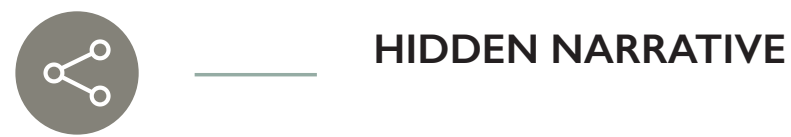

A Fishing site in 1990s

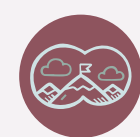

\section{VISUALISATION}

The site has a great view to

the lake, and the terrace allows

people to play and interact

with the water.There are no

sediments around the place,

which makes the water in this

area look crystal-clear.

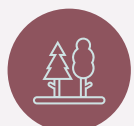

NATURAL

\section{ENVIRONMENT}

It only has few trees planted on the carpark space.

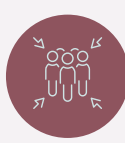

\section{GATHERING} SPACE

People can look at the lake by just staying in their car because the carpark is so close to the waterfront. Negatively, this stops people from actually experiencing the waterfront as a result.

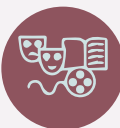

\section{CULTURE SIGNIFICANCE}

As a site used to be a fishing site, there are still some canoes left on the site, reminding us the fishing culture.
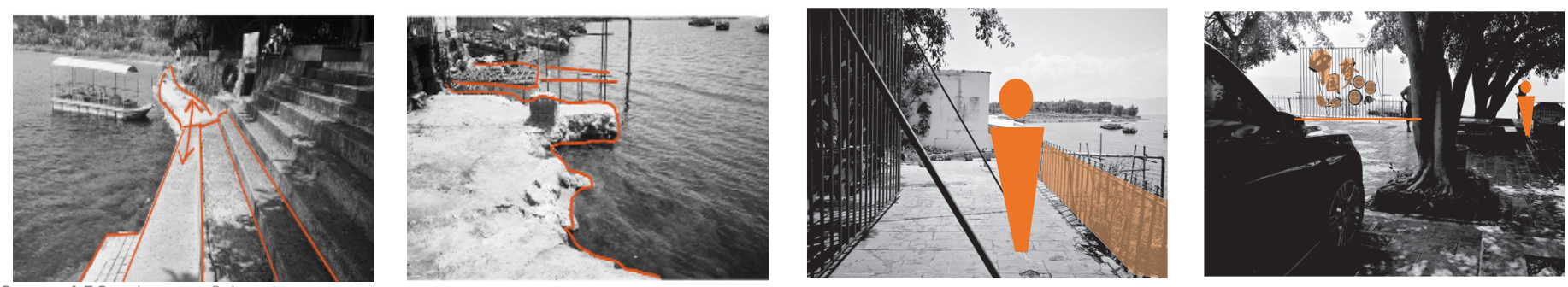

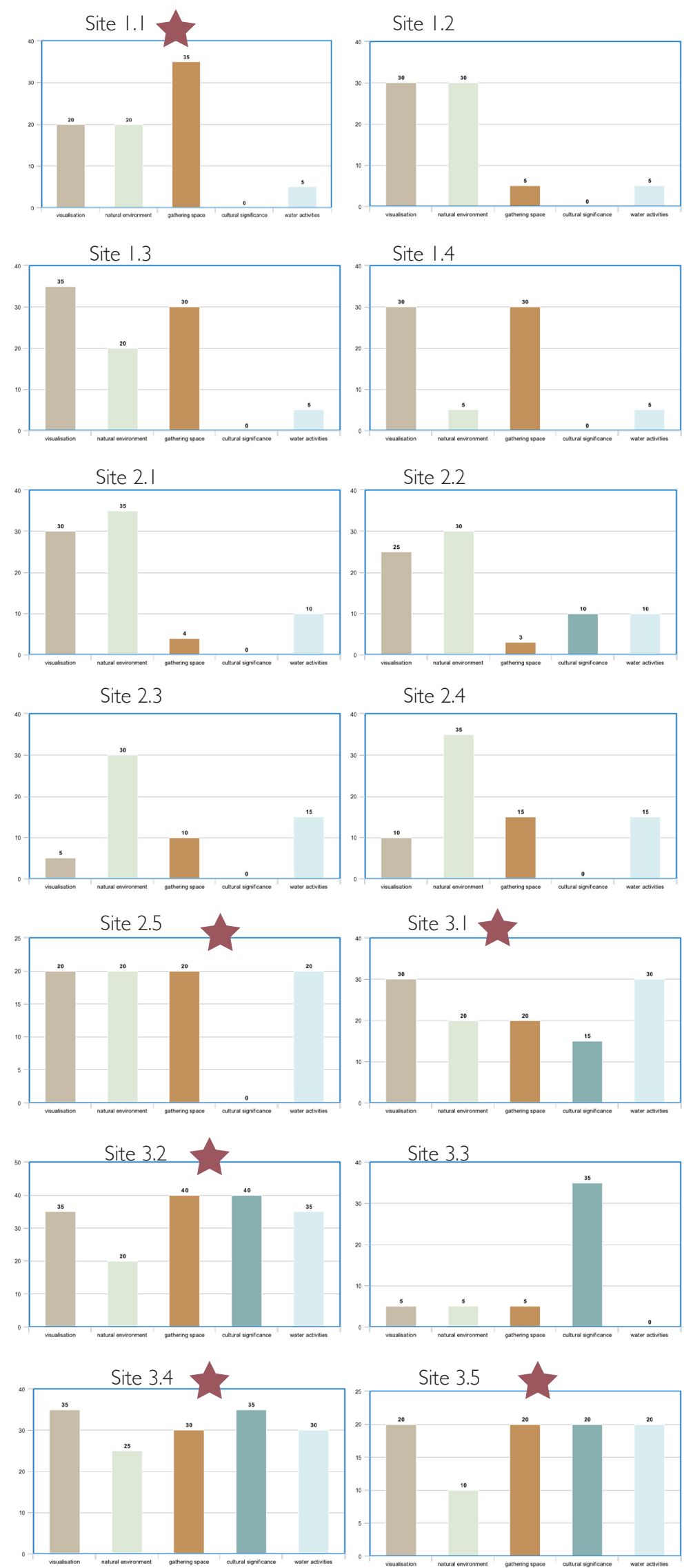

$\checkmark$ sites reach most of the design criterias

figure I6I, Data of I 4 sites

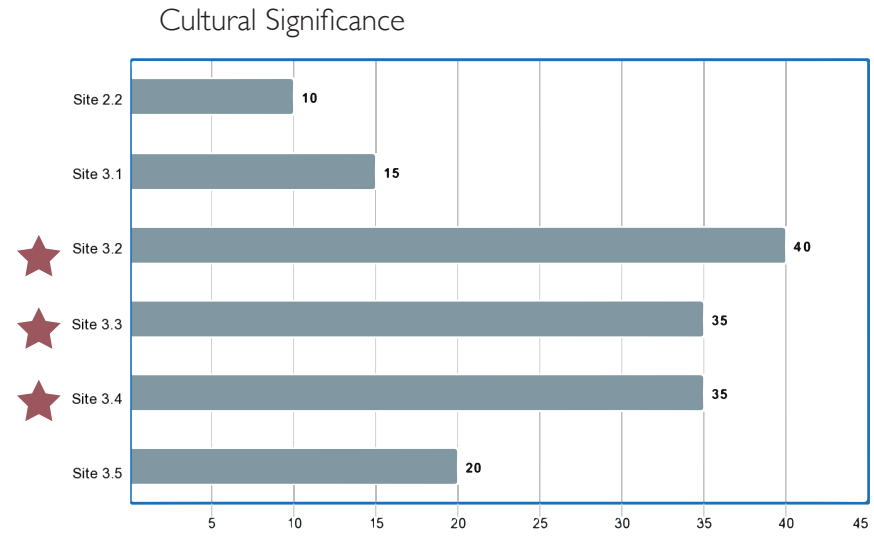

figure 162, Sites with cultural significance

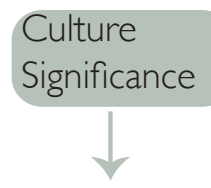

Visualisation

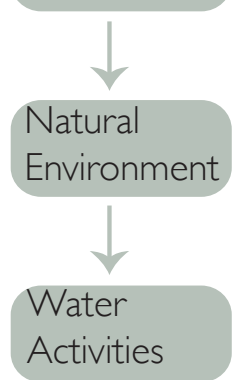

figure 163,

numerical order of site criteria
From the data anaysis(figure |6|) for 14 sites,most of the gathering space has exiting cultural values.

Then, the visual things is very important for design. For example, if a site does not have mountain views, it is not easy to bring natural mountains views from Landscape design.

As figure 163 shows, sequencing the data, the most important criteria is "Cultural Significance". After that, "Visualisation" is second important. Then they are "Natural Environment" and "Water Activities: 


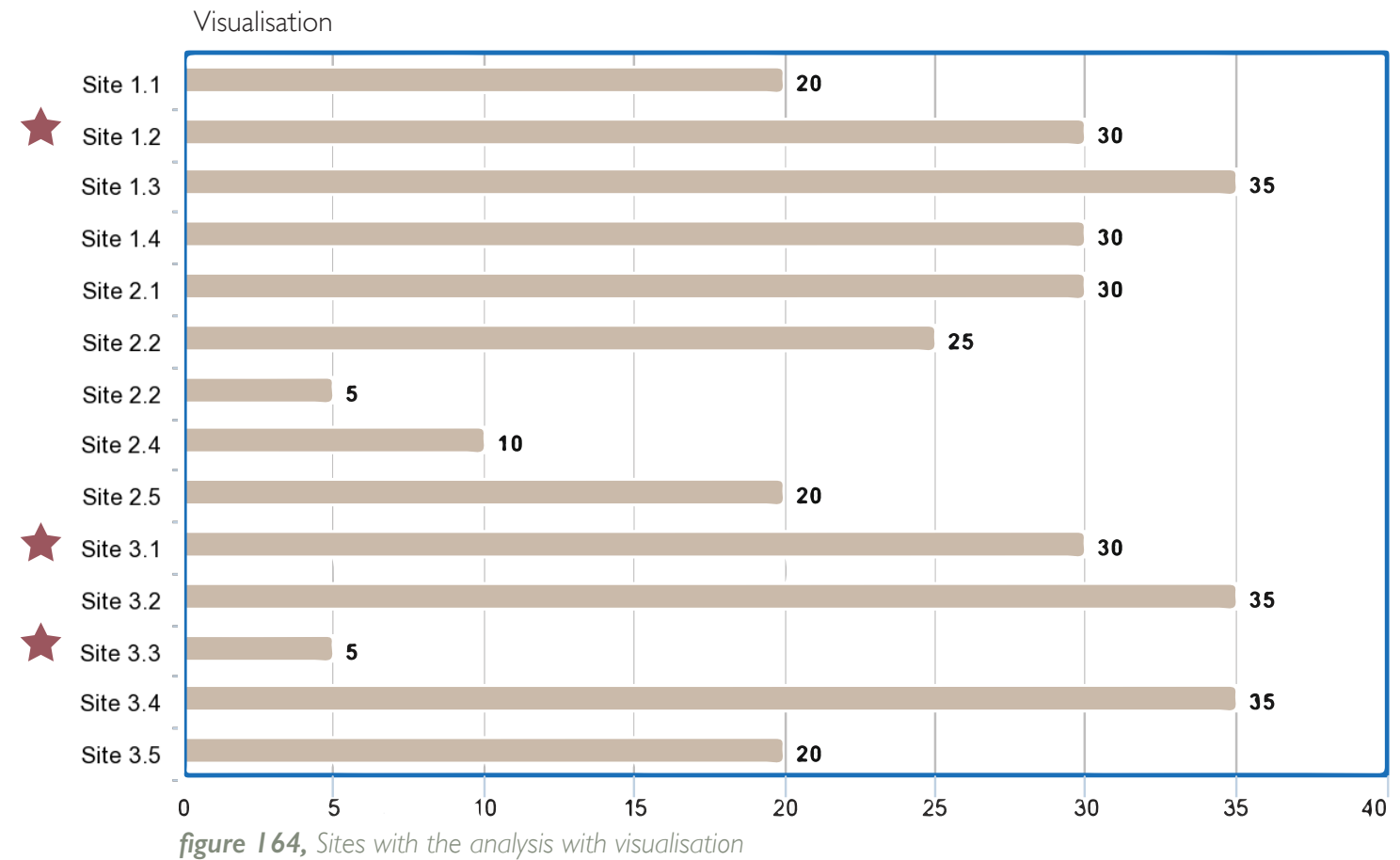

\section{Process for choosing sites}

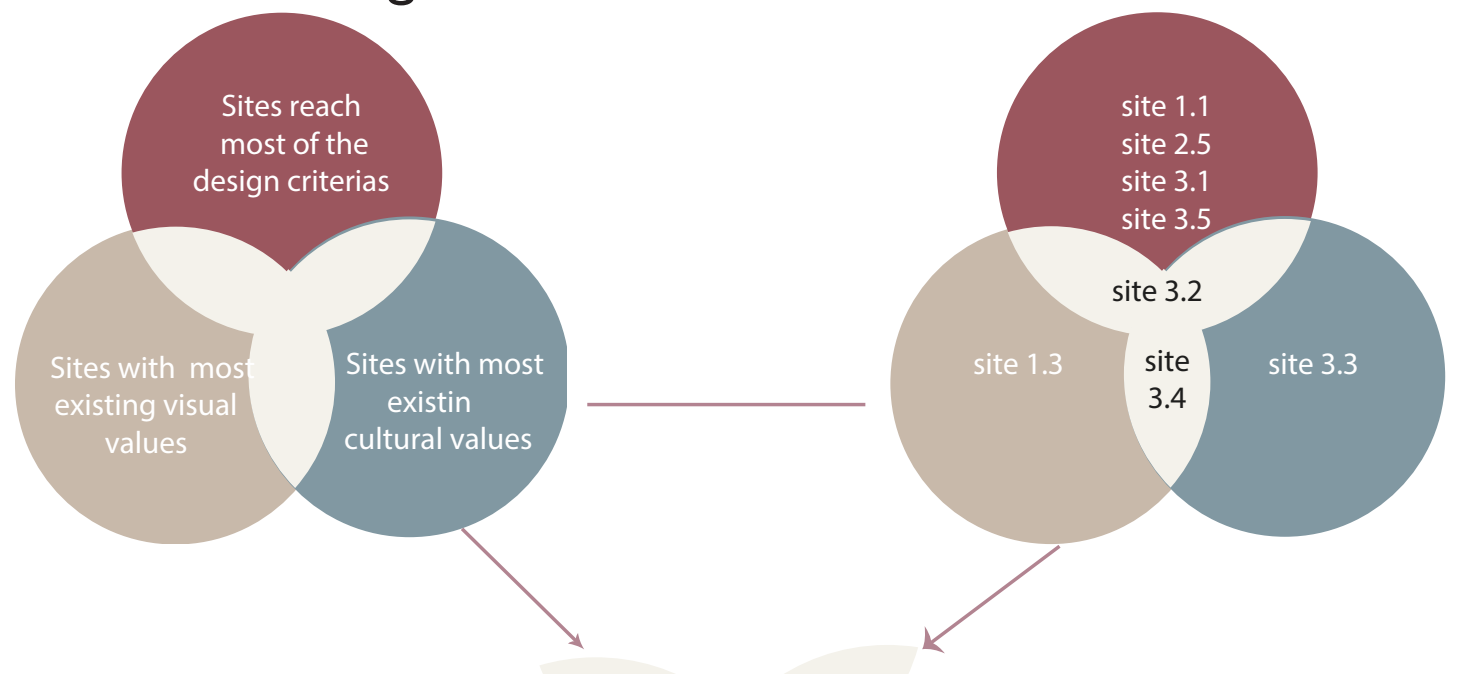

Site 3.2

Site 3.4

figure 165 , Process of choosing sites

Finally, after analysing the data. There are two sites coincide with "Sites reach most of the design criterias" , "Sites with most existing visual values" and "Sites with most existing cultural values" 
PRELIMINARY TESTING 

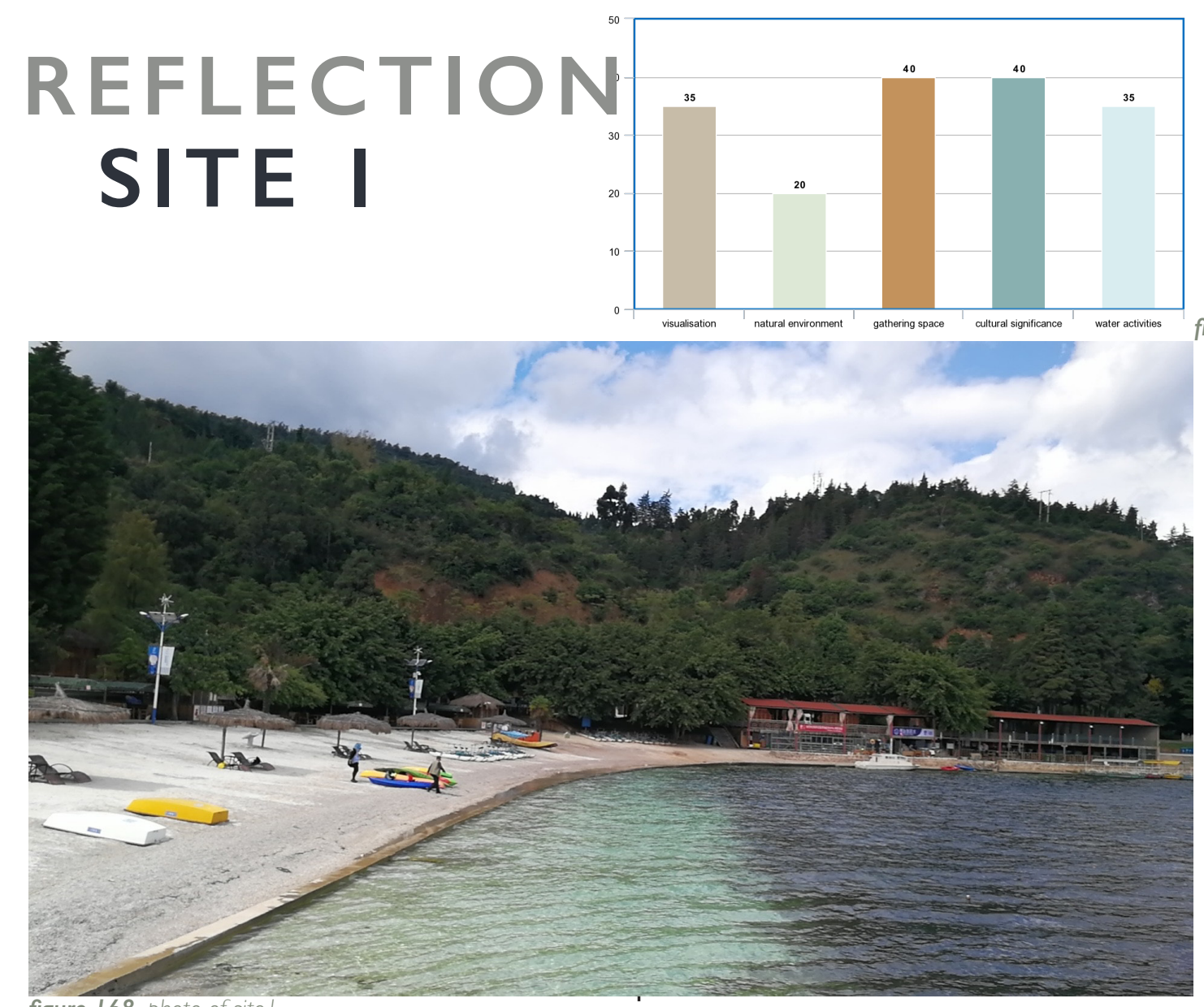

figure 168, photo of site I

Site one is the only one pakr has some feature of Dian culture around Lake Fuxian.

The testing aims to maintain the positive aspects of the sites, and reveal more hidden narratives of history and culture of Dian Kingdom.

The potential strategies will mainly be the methods that can create a landscape narrative. At the same time, allowing the design bring people a journey of Dian.

Then, to create a sense of place, "sight" ,"smell" "touch","sound" and "preception" should be considered during the design process.

The Augmented Reality would mainly focus on the functions that can enhance with the physical landscape. 


\section{DESIGN STRATEGIES BASED ON FRAMEWORK}

\section{Primary}
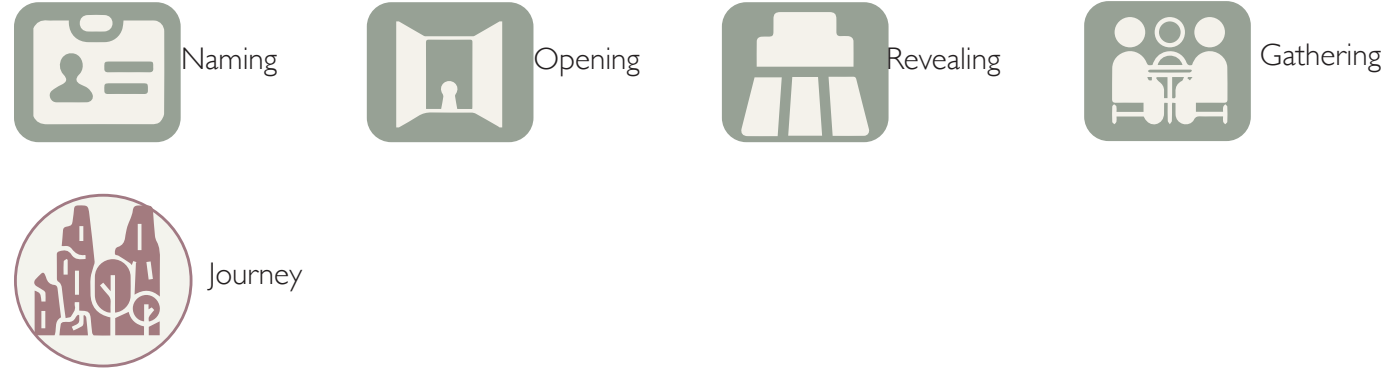

\section{Secondary}

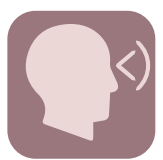

sight

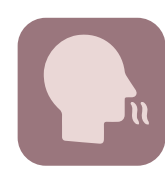

smell

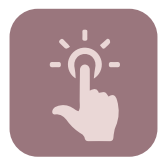

touch

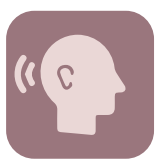

sound

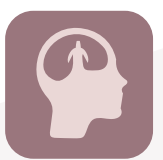

preception

\section{Augmented Reality}

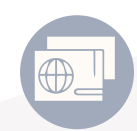

Identity

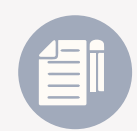

Create

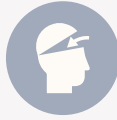

Impression

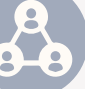

Social

\section{POSITIVE}

- Exiting community gathering space

- Exiting Dian cultural aspects

- open views of Lake and surrounding mountains

- Close to Underwater Structure Site

\section{NEGATIVE}

- Some unused space

- Few existing vegetation 

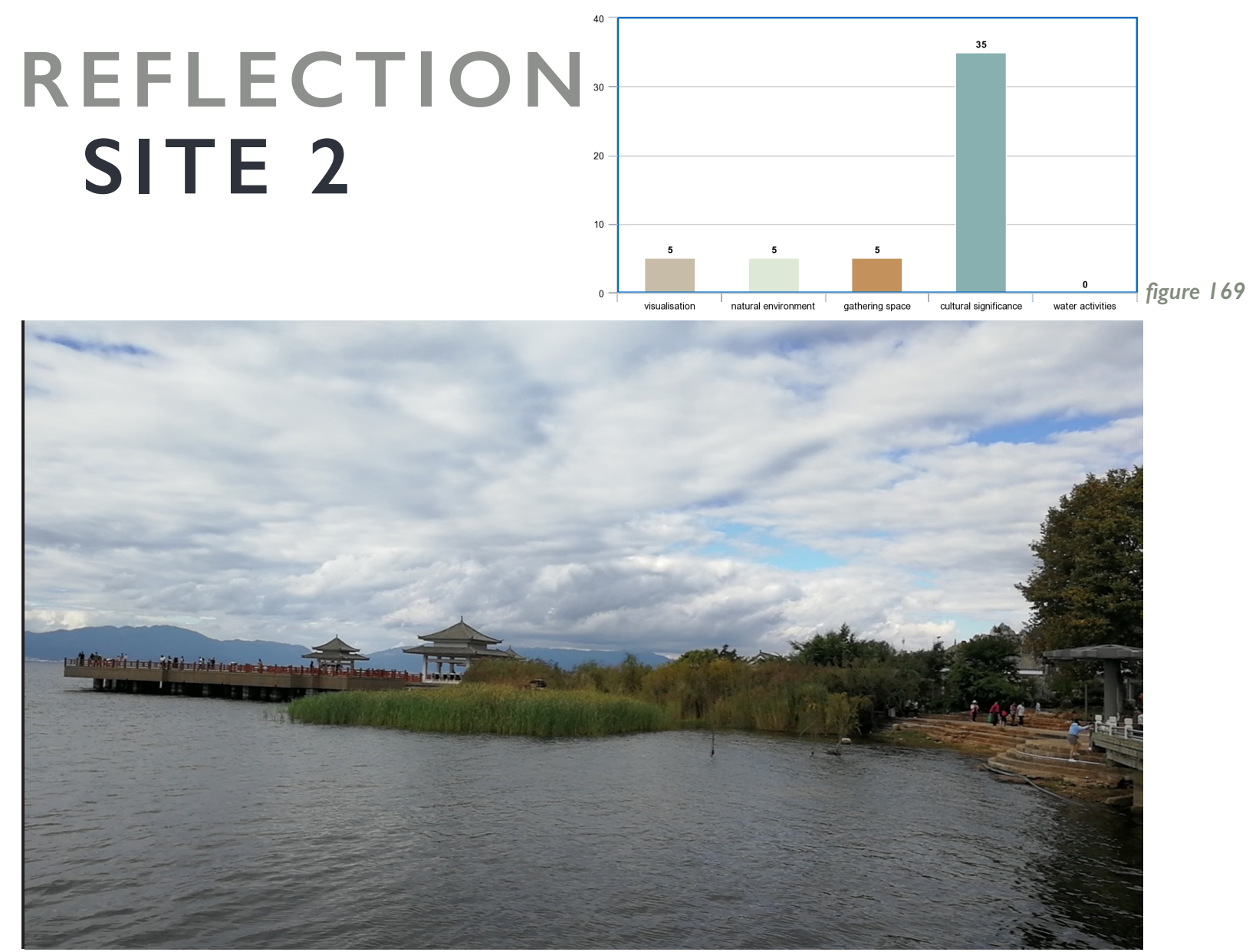

figure I70, photo of site2

Site current two has a bridge walkway that allow people to walk on the bridge and view the lake.

The potential strategies will mainly be the methods used in traditional Chinese garden. It also will focus on human senses "sight" ,"smell" ,"touch","sound" and "preception".

Then, to design a garden with Chinese features,"Fengshui" "Wuxing (five elements)" have to includes to the design.

The Augmented Reality methodology of site two is potential same with site one. 


\section{DESIGN STRATEGIES BASED ON FRAMEWORK}

\section{Primary}

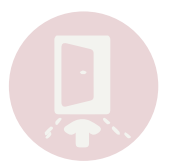

penetration

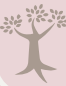

reveal

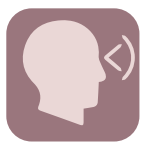

sight

Secondary

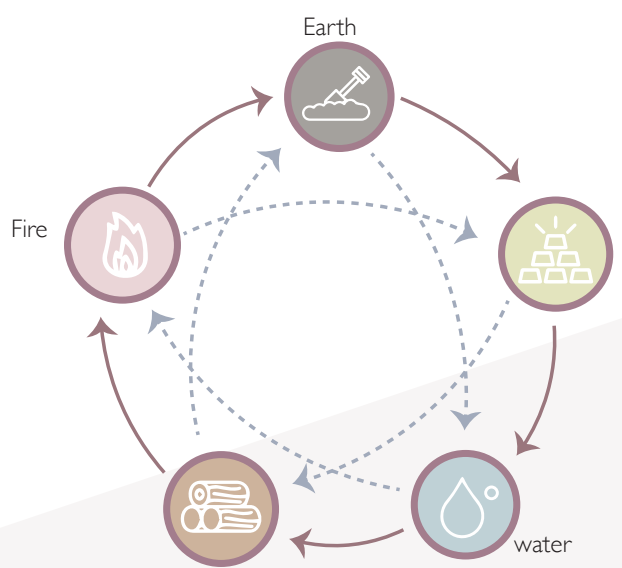

POSITIVE
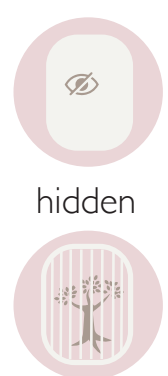

shallow reveal

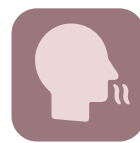

smell

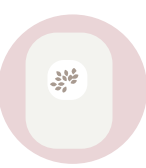

shallow hidden

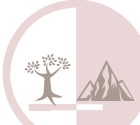

suddenly change a sene

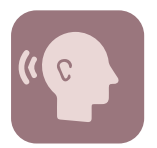

sound
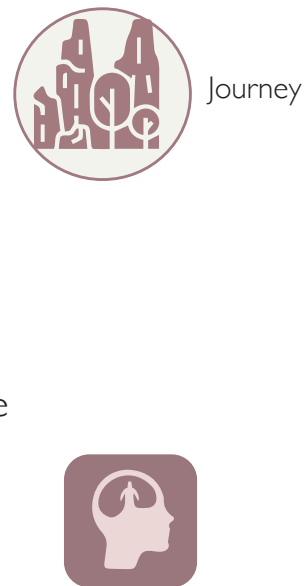

preception

Augmented Reality

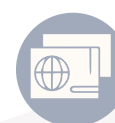

Identity

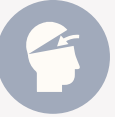

Impression

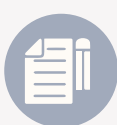

Create

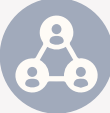

Social

\section{NEGATIVE}

- has a sense of Chinese culture

- with existing marble materials

- Has natural intereting pattern of the stone

- Close to Underwater Structure Site
- Loss of connection with water

- Regular Chinese Features 


\section{ITERATIONS}

The following iterations will be testing spatially key ideas, such as, landscape narratives, multifunctional uses, attractive and inviting space for AR, cultural presence, ecological diversity and the connection of people. The overall goal is to focus on designing a landscape narrative by testing how to incorporate a sense of place to the physical world while simultaneously testing how augmented reality can enhance the design.

Looking into the idea of adaptive use, these chosen iterations (Fig) take advantage of multiple experiences that visitors can explore around the lake. Using the landscape to highlight specific sites of interest, the design aims to incorporate cultural significance and ecology through layers that can only be achieved with AR 
For AR, people can easily download an application in their phone when they scan the code on the site. The free wi-fi will cover on the whole site to make sure people can load the AR model on their phone.

Framework reflection:

To back-up and reassure the design is delivering in its purpose within the research; the key ideas (Fig. ) to give clear criteria and intention for all design moves.

This step is a crucial part of the design process, as it connects the design section with the previous research analysis. This process helps the researcher to gain an understanding of what the design strategies should include. It is equally important to reflect and critique each iteration in its connection to the framework, to allow for further development and a perfect final design. 


\section{ITERATIONS OF SITE 2}

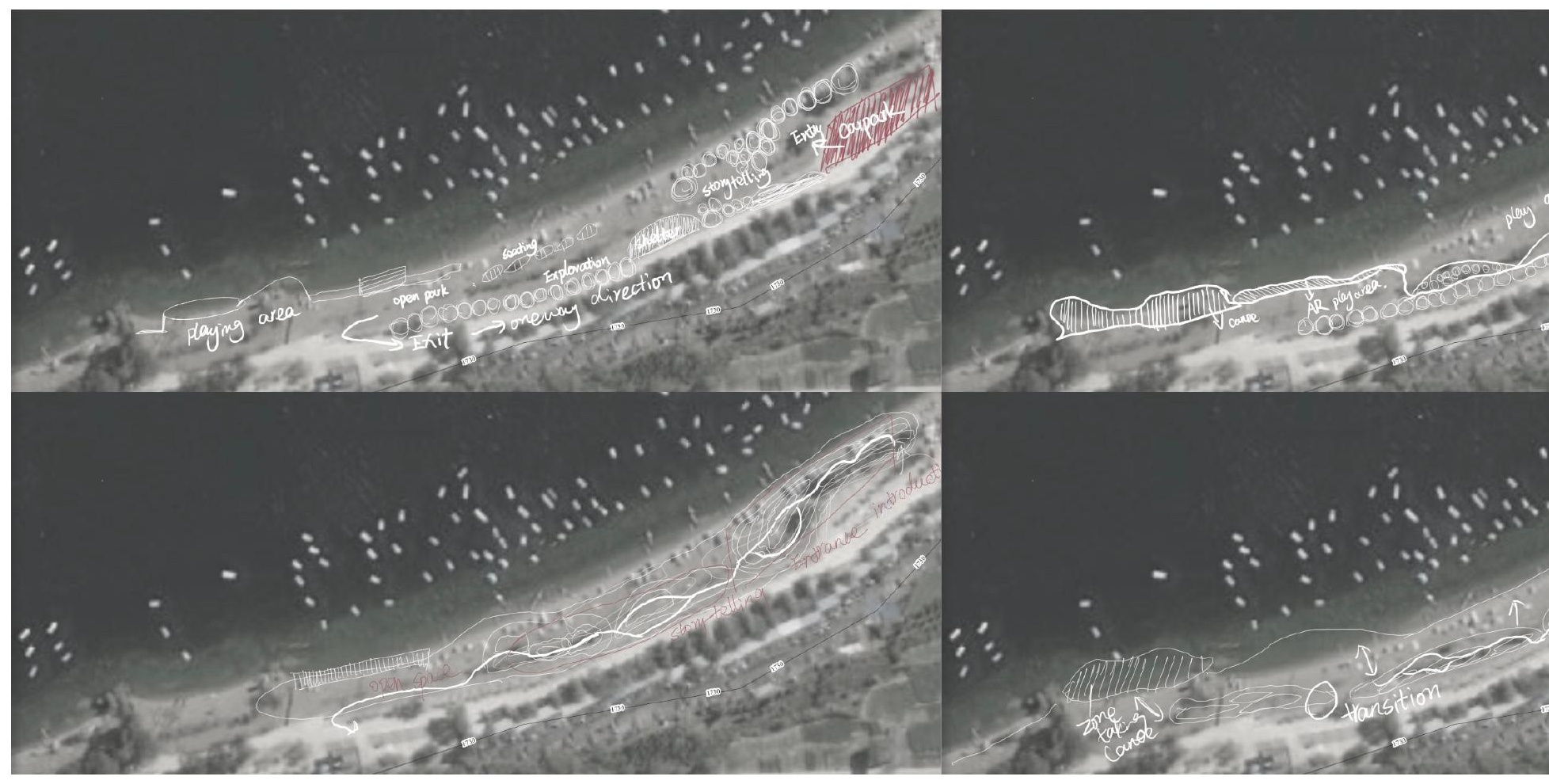

CHOSEN ITERATION 

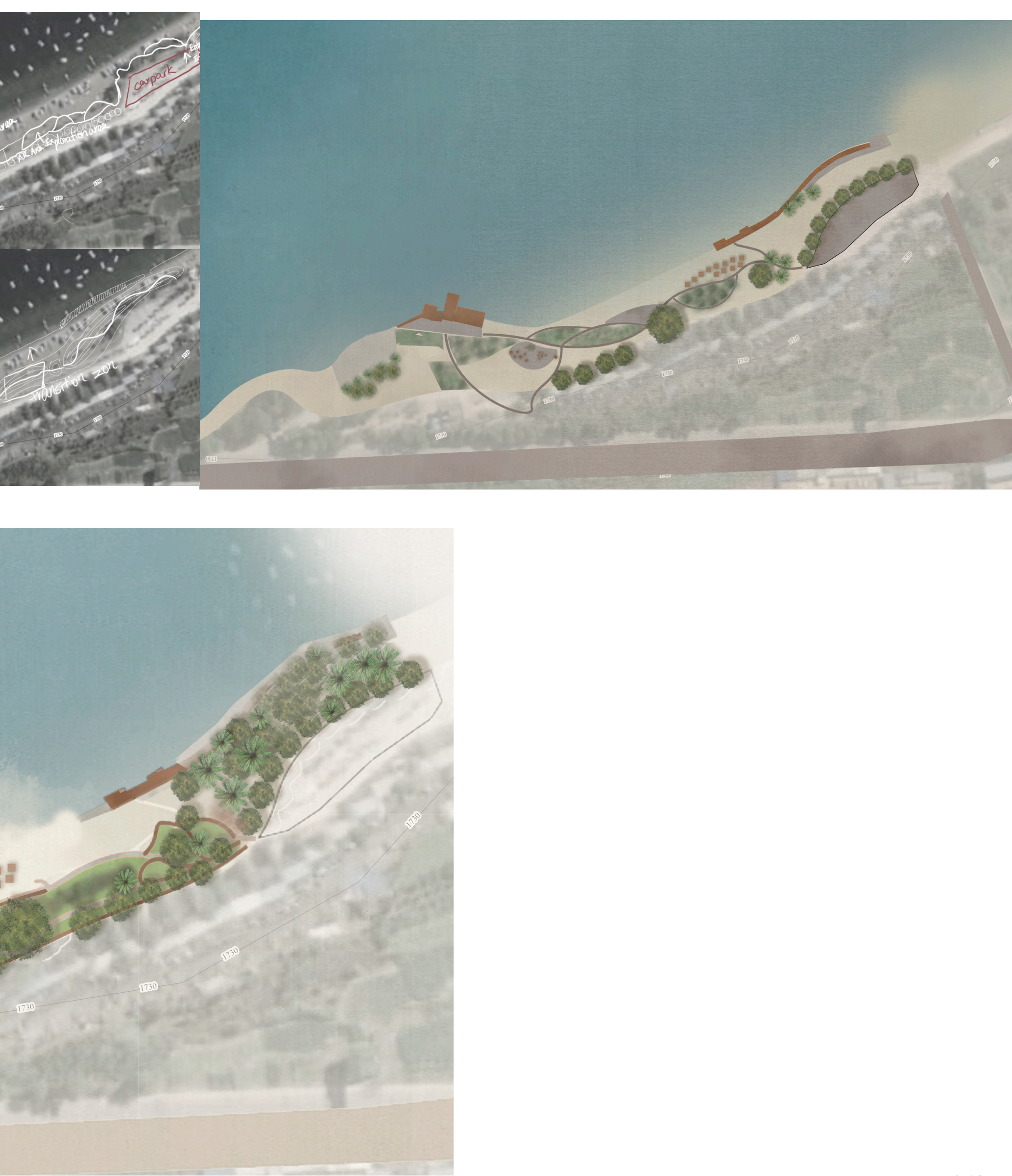


\section{ITERATIONS OF SITE 2}

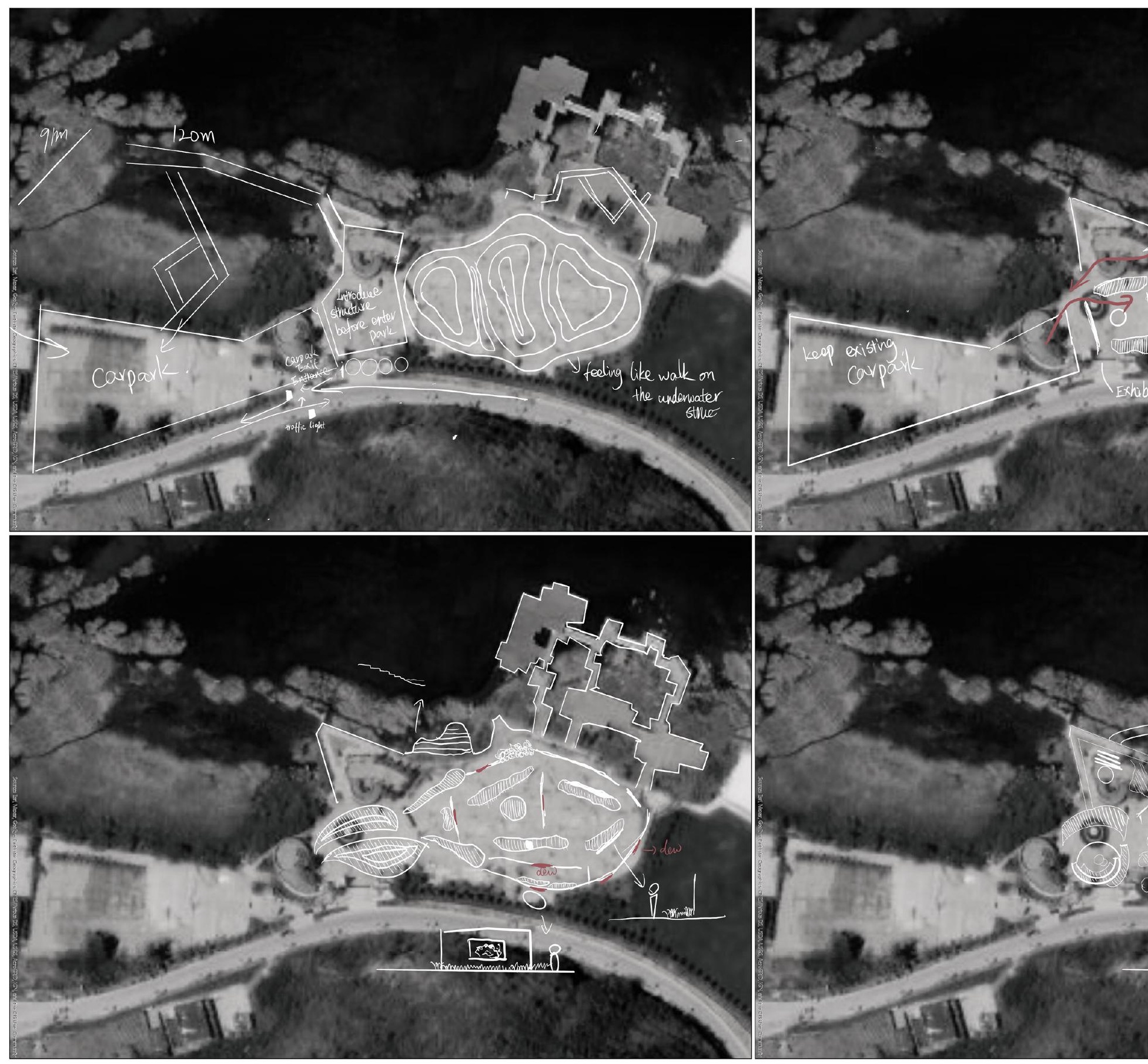

By sketching over the top of maps, including the motif o the final design has improved with more attention framework. Many additional ideas were able to be fur such as telling stories in different parts and planning enhance the a famous Dian story. 


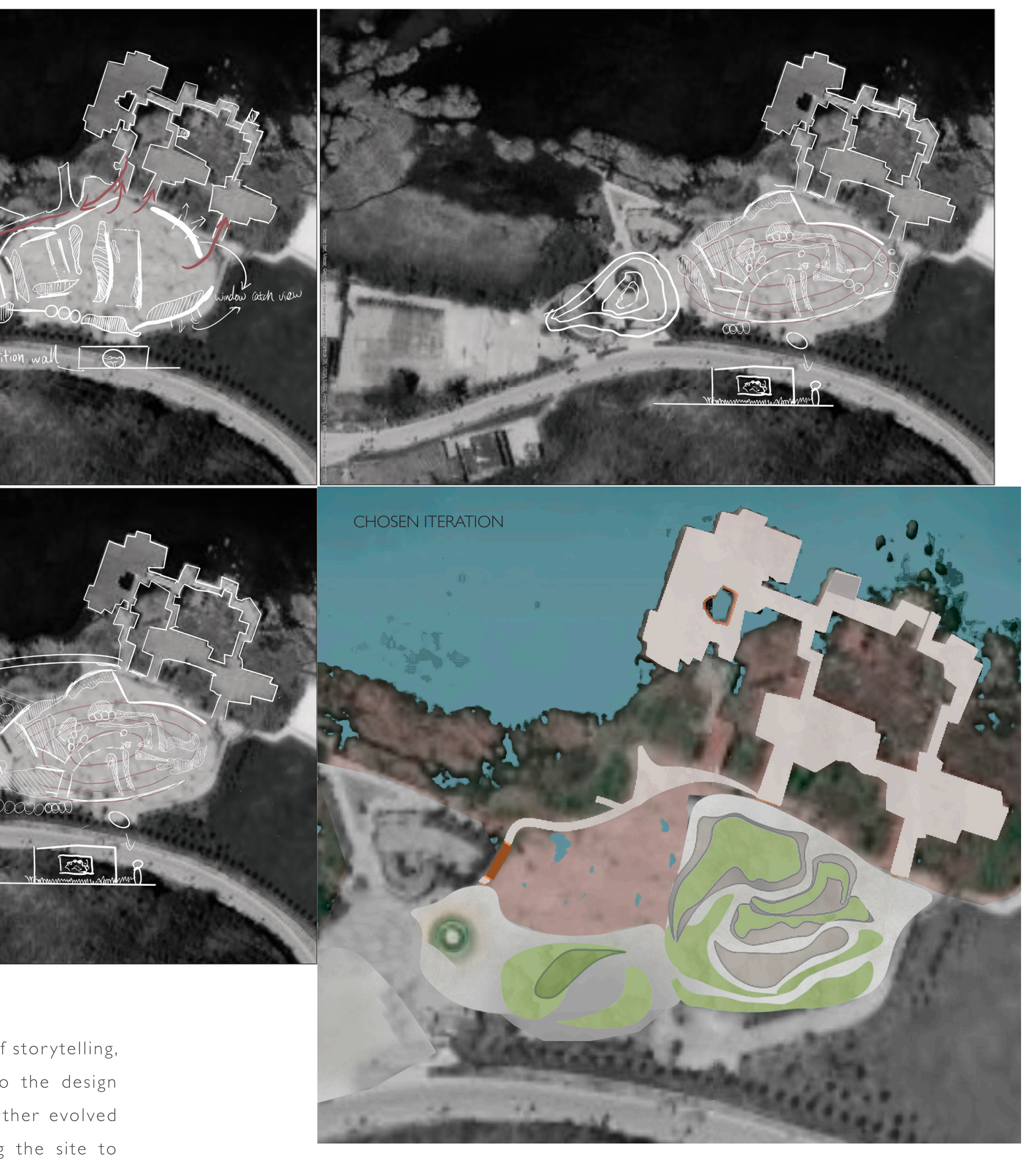


DESIGN

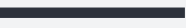


SITE I

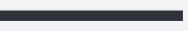




\section{Design of site I}

Site one aims to be a place that celebrates Dian culture and can educate people through the many facets of this ancient civilisation. As some aspects of Dian culture is intricate and expensive to rebuild in the physical world, therefore most of the Dian characteristics will be shown within AR. The design aims to enrich Dian cultural value through narrative landscape design which is enhanced with Augmented Reality.

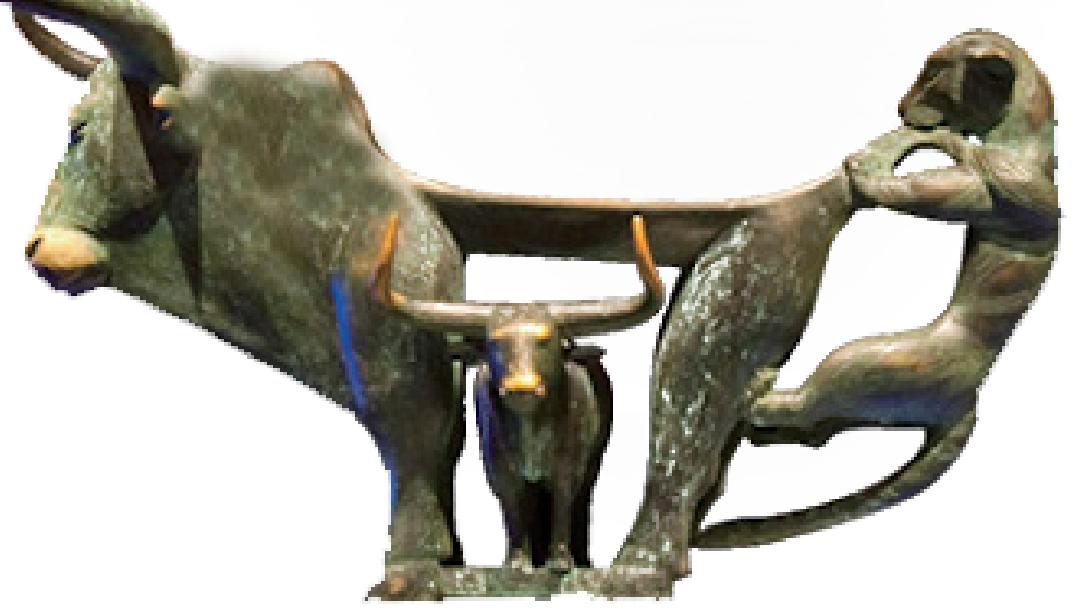

Whenever people refer to the Dian Kingdom, most people recall the bronze drum and the techniques used to create it. The most famous bronze of Dian is "an Ox protecting its calf from a tiger", it demonstrates both the culture of Dian and Taoism. Also, it is identified as the most famous antique in Yunnan Province (Yishiweijian, 20।8).

The story that is illustrated by the bronze statue: A tiger is biting the tail of an Ox, intending to kill both the adult and the calf it is protecting (Yishiweijian, 20।8). The story has an open ending, allowing people to imagine what will happen in the next moment. The protection of the calf reflects how a mother defends and shields her baby and the actions of the tiger show the concept of survival of the fittest, from Taoism philosophy (Yishiweijian, 2018). 
The experience of the design is based on the story of the Ox bronze statue, where users are treated as the calf. There are three changes in mood as the calf gets protection from its mother. The first one is the tiger found the calf and its mother, and they are escaping to find a hiding place. Secondly, the $O x$ is slower than the tiger, and because of that the mother takes care of the calf. At this moment, the calf might not know what is happening to its mother, because the mother is holding the calf in her arm. The third change in mood is the calf's mother gets seriously injured after the attack, and the calf noticing that the mother is getting weaker and that itself needs to be stronger, as a form of self preservation, to protect both the calf and its mother (Yishiweijian, 2018).

By treating the users of the design as the calf of the bronze, the users experience the park into three main parts. The first, is feeling unsafe because a tiger is chasing them. The second part is feeling protect as their mother defends against the attack. The third part is feeling a bit unsafe and becoming mature within the environment.

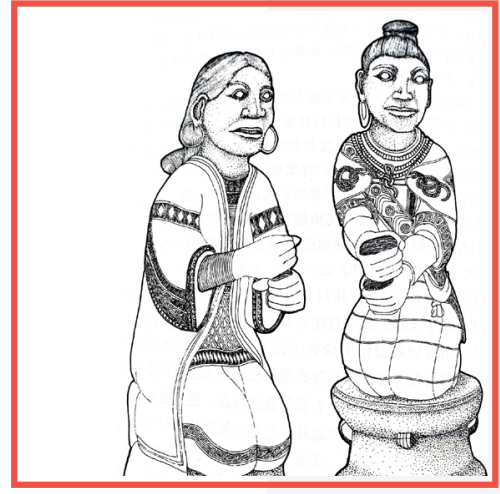

figure 176, Dian Clothing

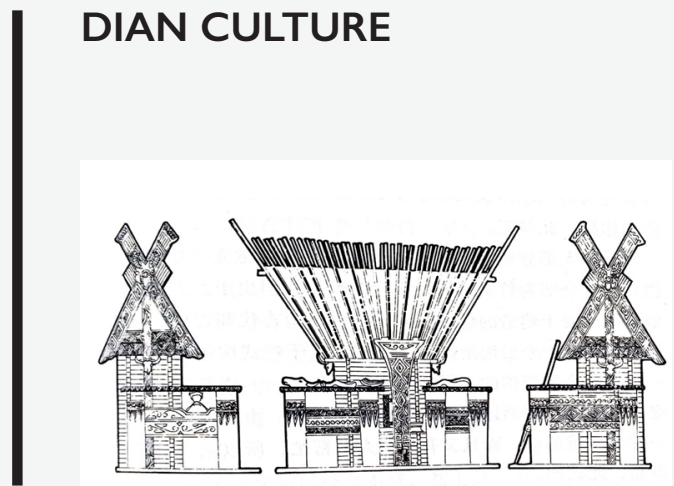

figure 177, Dian Architecture 


\section{MASTER PLAN}

pathway

„unu,u, slow movement area

mumum entrance area, fast movement

exploration area, medium speed movement

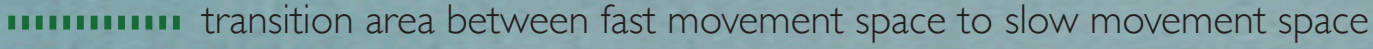

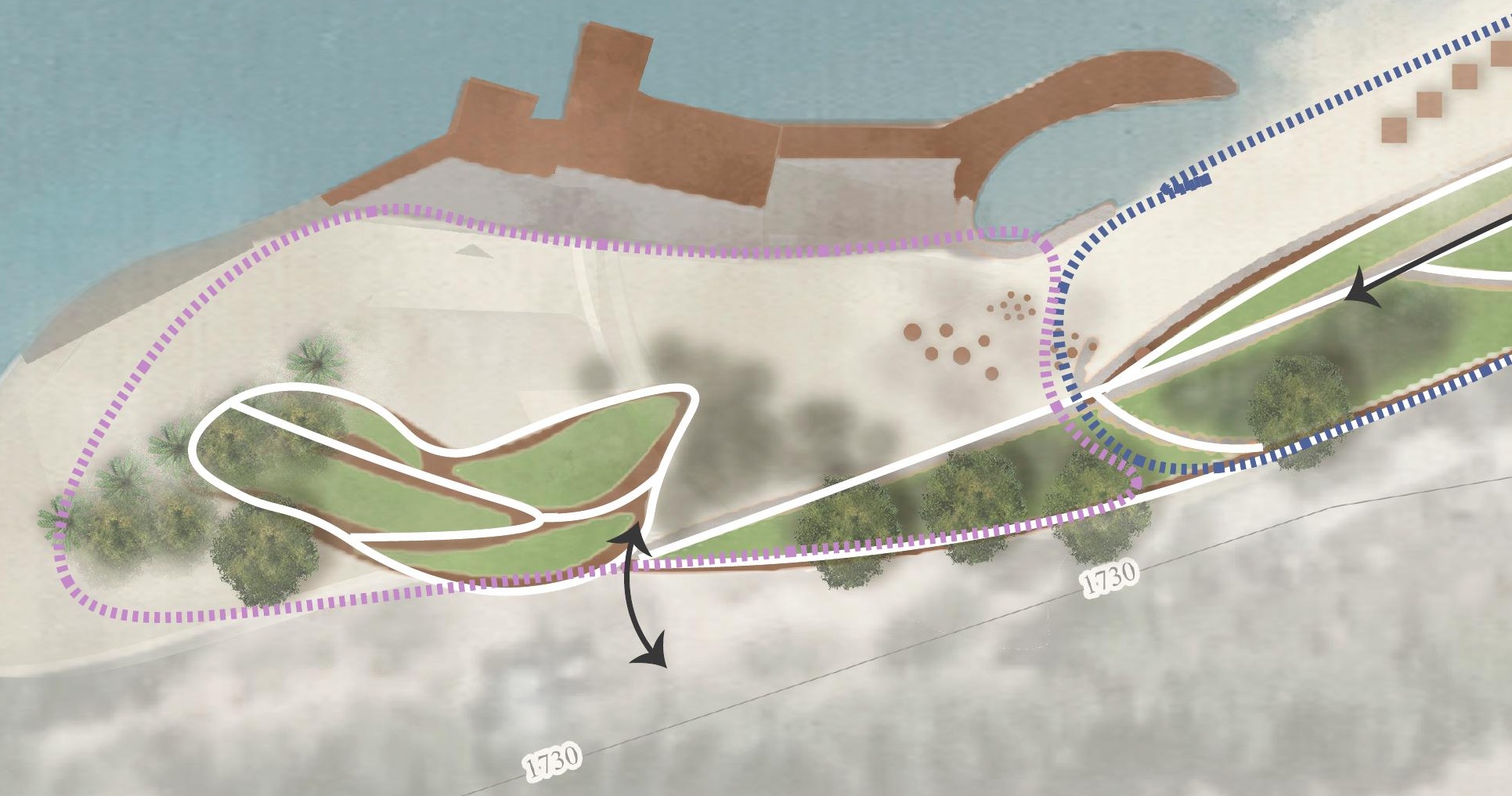


MASTER PLAN WITH DESIGN METHODS
rat
回
IIIt

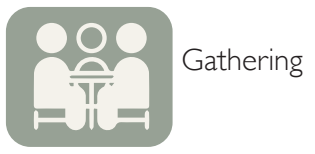

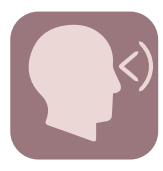
sight

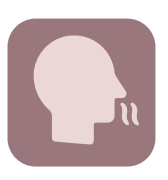
smell

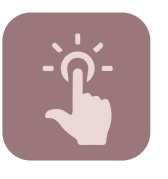
touch

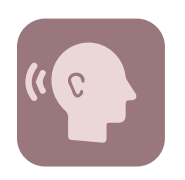
sound

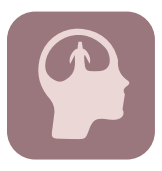
preception

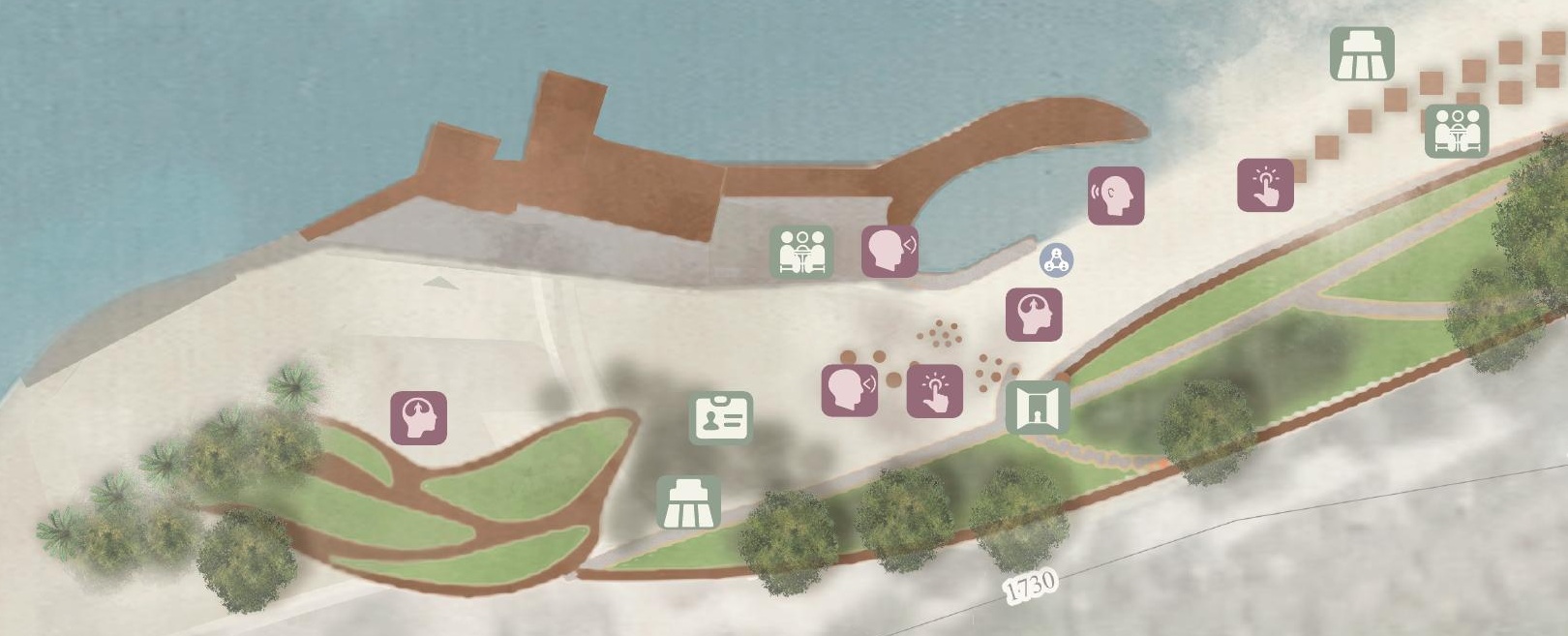




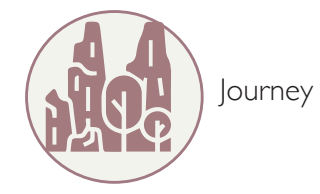

\section{Augmented Reality}

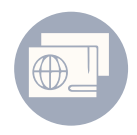

Identity

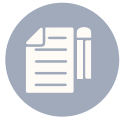

Create

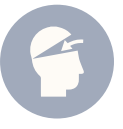

Impression
8

Social

0 (290 113 $i=$ 
SITE 2

- 
This site is unique and close to the underwater remains. People are not allowed to dive or swim in the Lake, unless they have a freshwater diving certificate. The bridge has some features of Chinese Architecture that create a sense of place and cultural connection. The primary material of the existing bridge is white marble as the site is only $30 \mathrm{~km}$ away from the origin of the marble. Also, the white colour of marble can be a tool to design a Chinese garden, as a contrast with other colour(Wenku, 2013).
The historical narrative of fossils is significant to the Lake, but locating the fossils is difficult. Thus, there are opportunities to bring the knowledge of fossils to all visitors at this site. Therefore, the users of the design will be treated as if they are walking amongst the underwater remains.

The Landscape will demonstrates the meaning of the name of the county Chengjiang(figure 180).
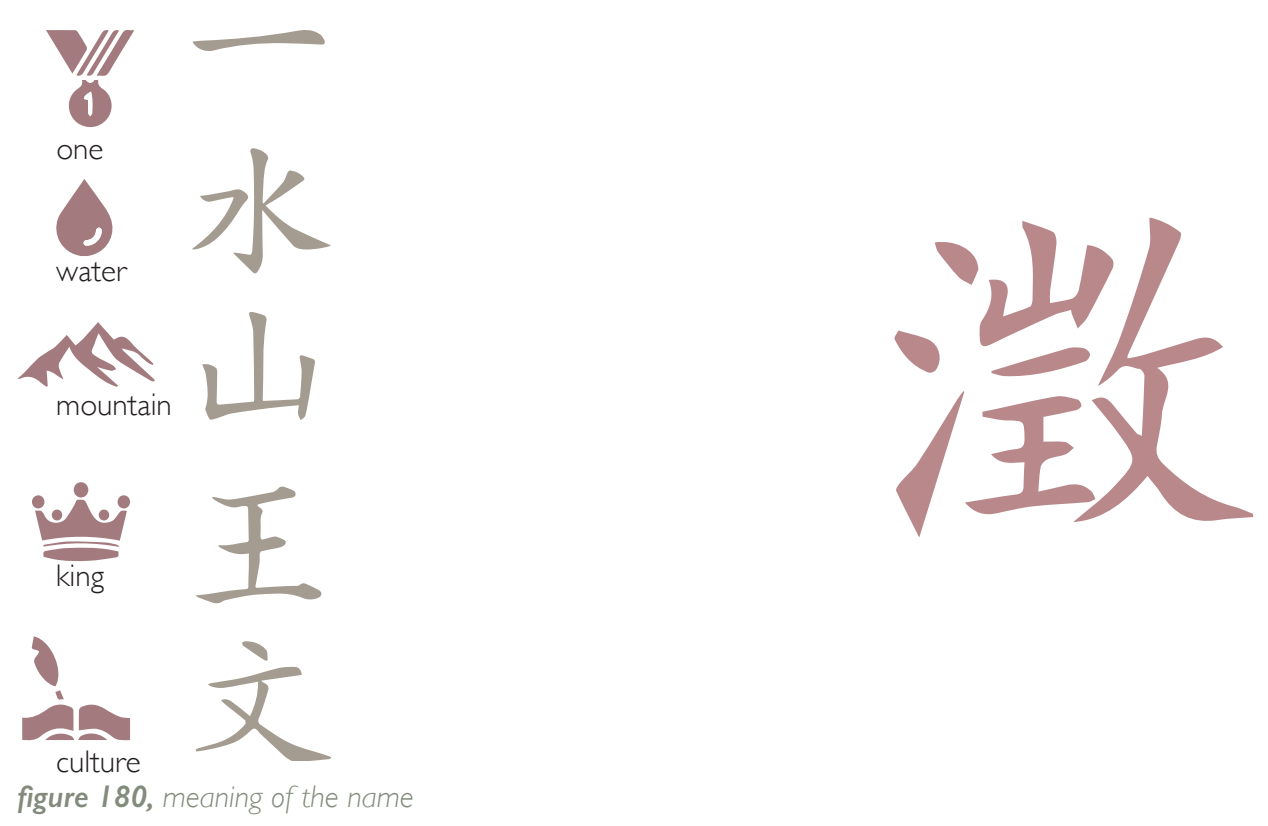

figure 180 , meaning of the name 
The age of the underwater remains according to material analysis, sets its origin to around 1800 years ago. During that period, Dian was under the management of the Chinese government. Therefore the design will let people know how Dian and Chinese culture combined through landscape. During the underwater structure period, the traditional Chinese gardens in China were mainly for hunting wildlife. Then, to use people experience the history in 1800 years ago, has concept for hunting the parts of underwater structure and paleontology found around the Lake through Augmented Reality.

Inspired by the Lion Grove Garden from the precedent review, the same methodologies that were utilised with in the garden to portray a narrative, will be used in the design(figure 180). As a design with Chinese features, it should follow the Fengshui of Chinese philosophy, which include the five elements metal, wood, water, fire, earth.

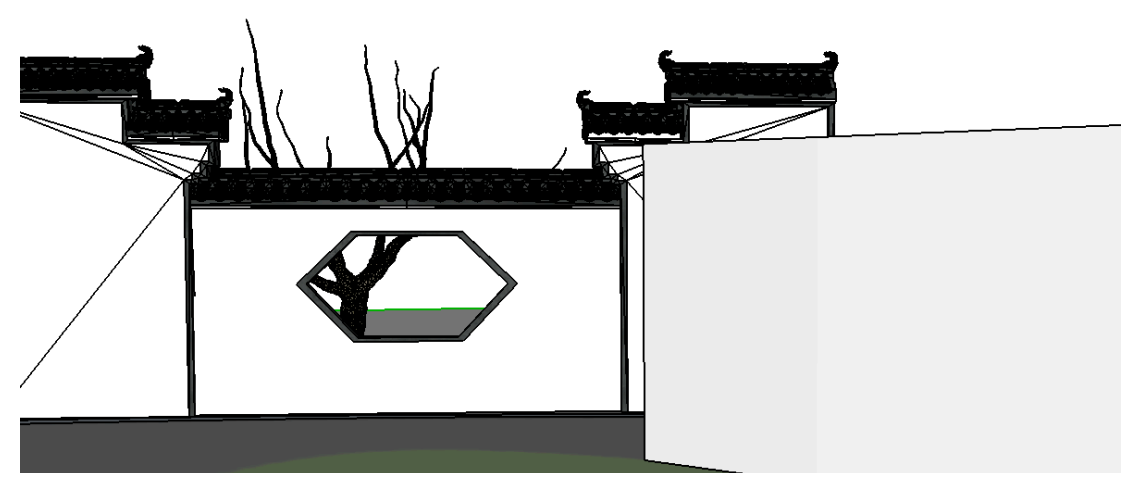

figure 180 , methods of narrative 


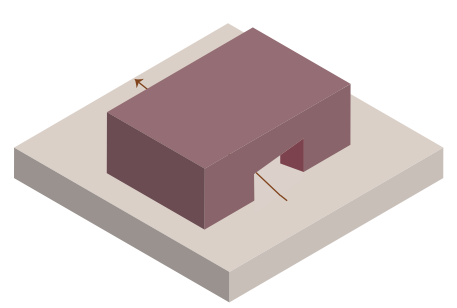

penetration
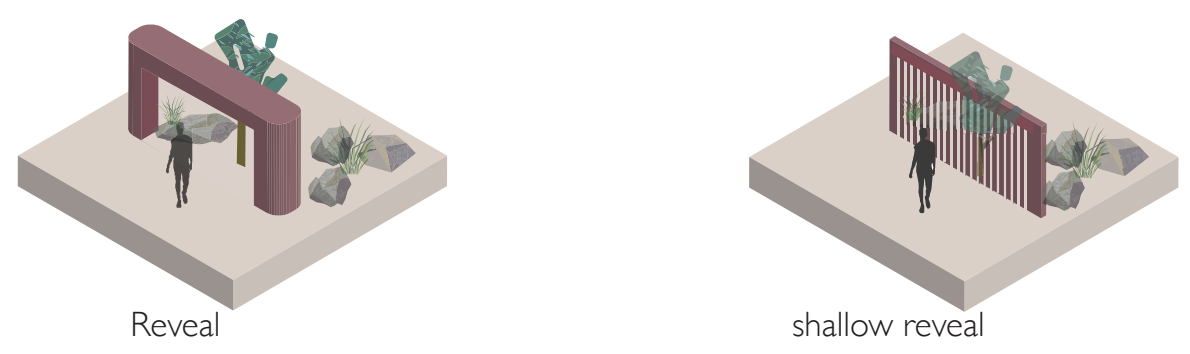

shallow hidden

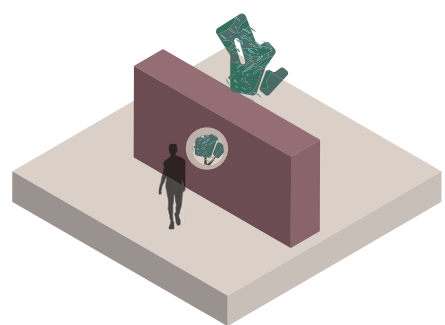

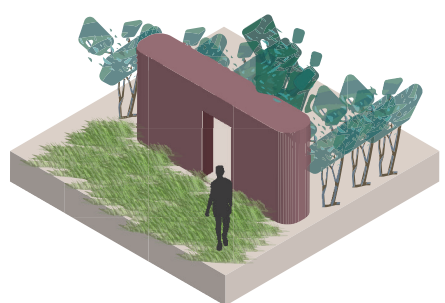

suddenly change a sene

figure $18 I$, methods of narrative 
welcoming area

exploring area

closure space(relevant private area)

transition space( exhibition area)

existing walkway

1 windows
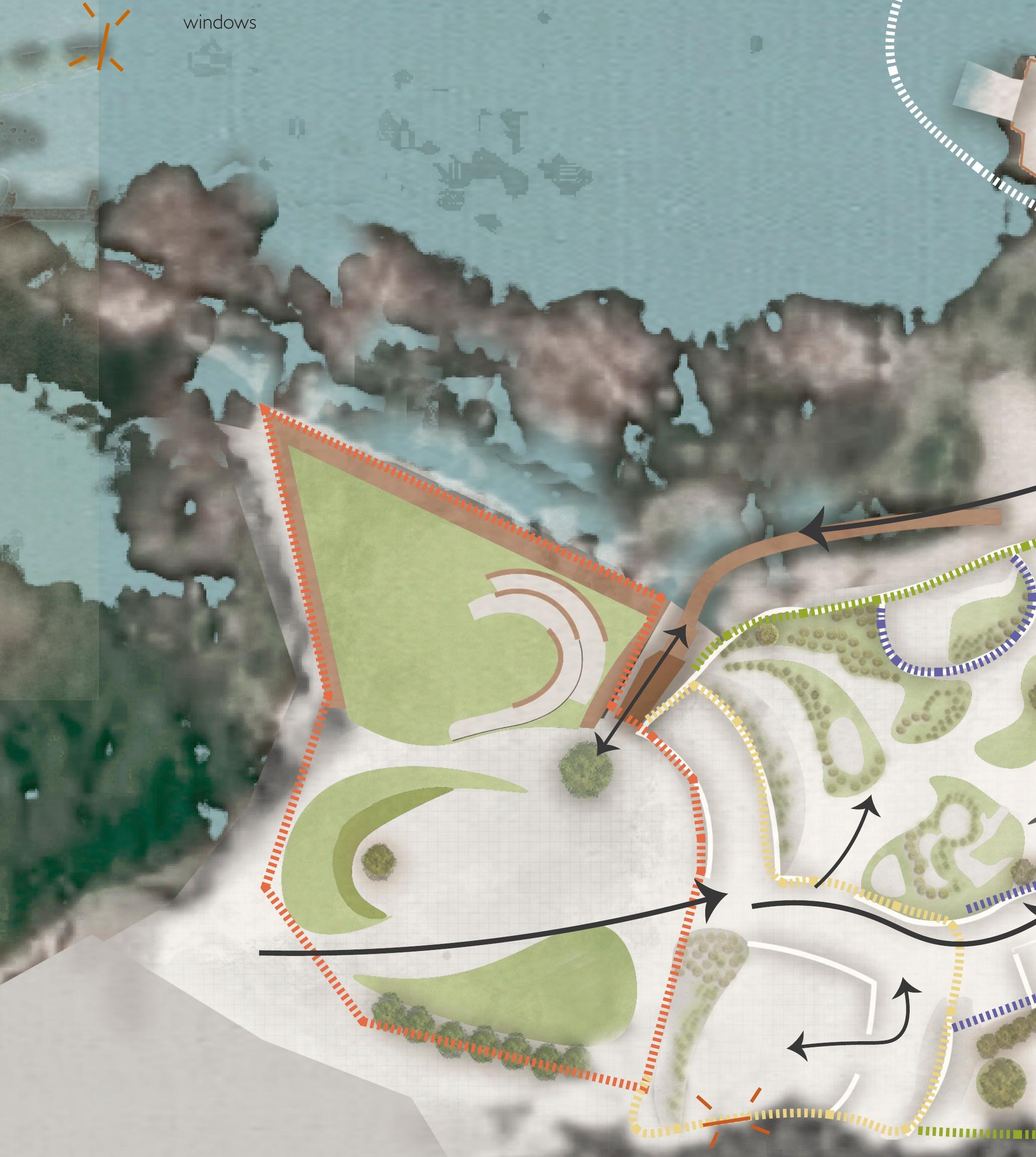


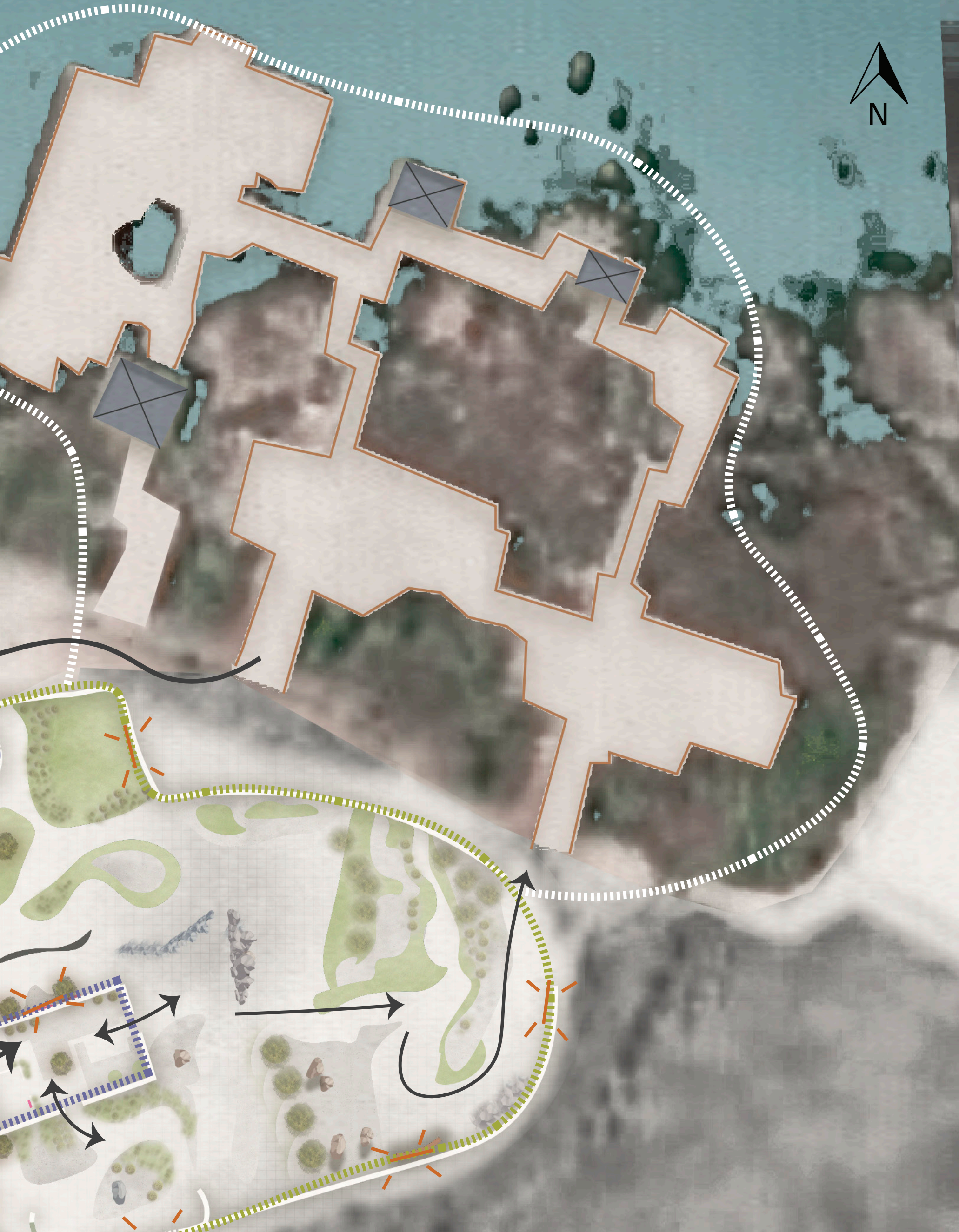




\section{MASTER PLAN WITH DESIGN CRITERIA}

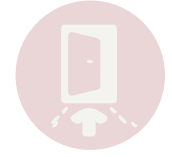

penetration

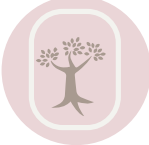

dew

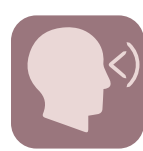

sight

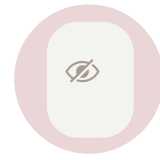

hidden

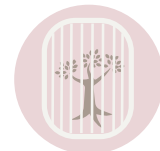

shallow dew

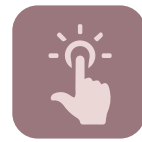

touch

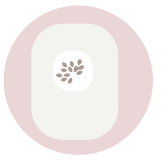

shallow hidden

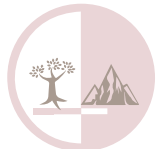

suddenly change a sene

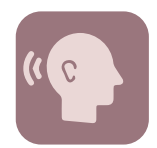

sound
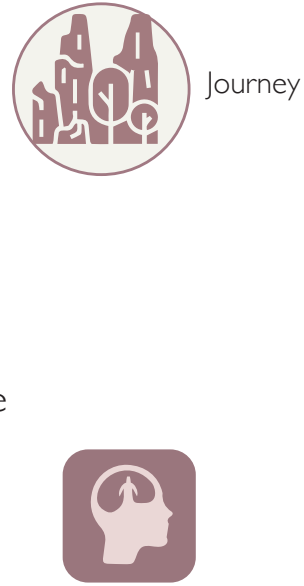

preception

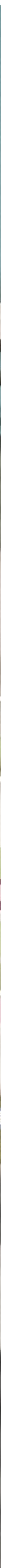



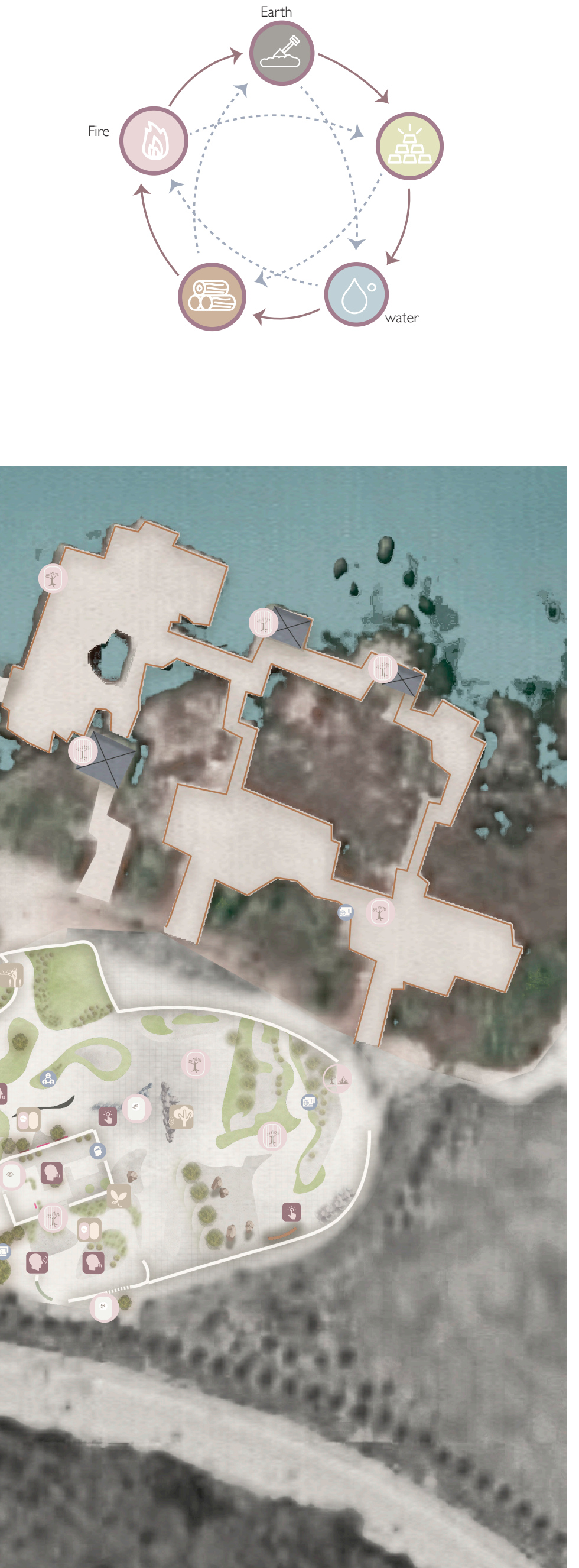

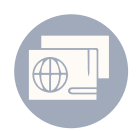

Identity

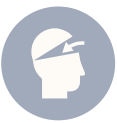

Impression

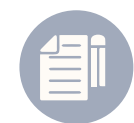

Create

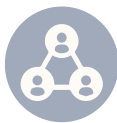

Social

Because there are a total of five levels of the underwater remains, the topography of the design will have five levels of changes. People will experience levelling up and levelling down, just like they are traversing through the real underwater remains(figure 182).

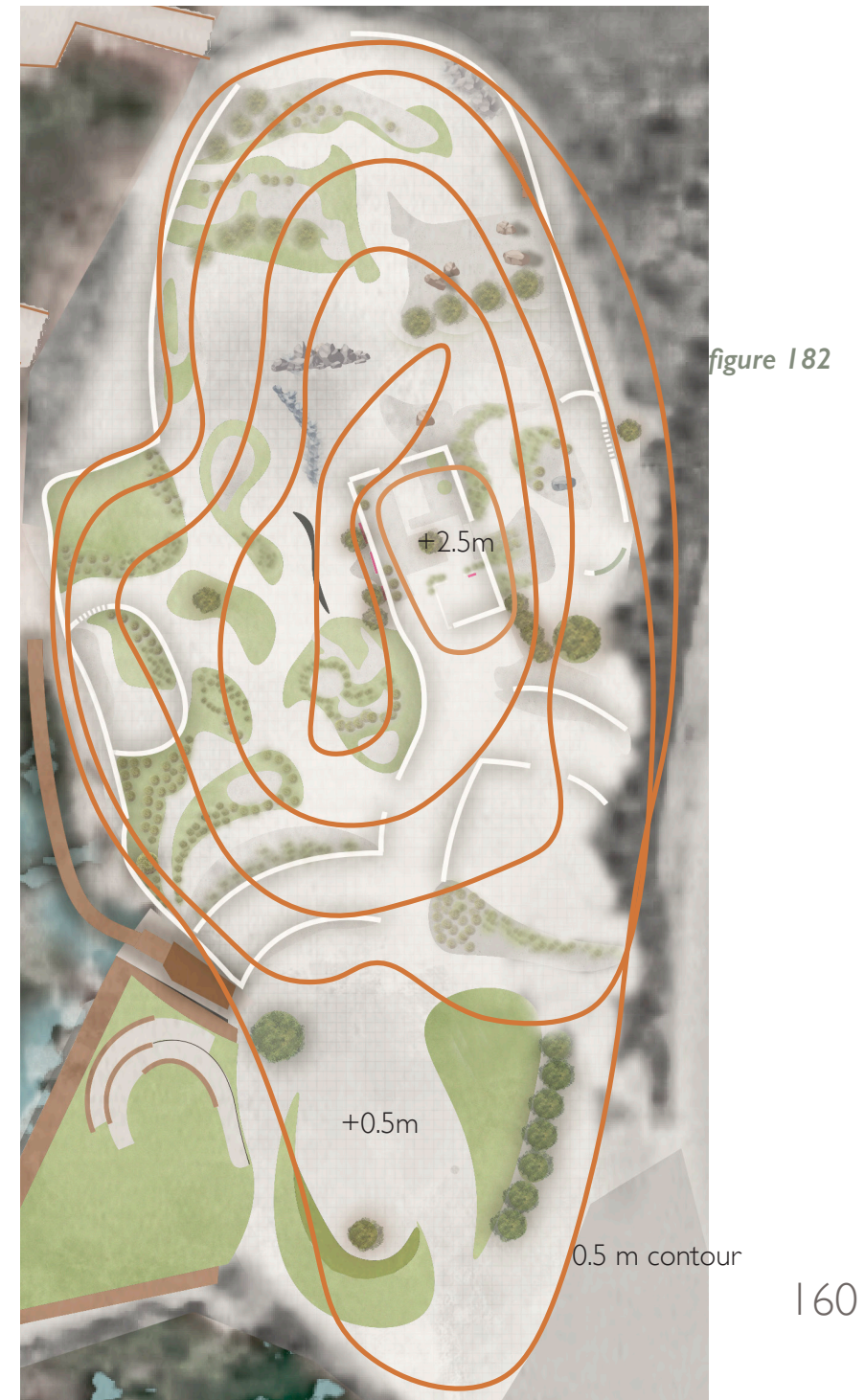


DEVELOPED DESIGN

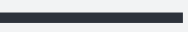


SITE I

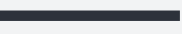


The detailed design will illustrate how it followed the design criteria that was concluded from the previous study. The detailed design is based on the framework, and will be introduced in different narrative methods including "Naming", "Revealing", "Gathering" and "Opening".

The inspiration of the shape of the pavement is from both Dian's Architecture and patterns on the material of Dian drums(figrue 183). In Dian culture, almost all of the patterns are made by triangular shapes, which is also seen within the architecture The length of the triangle is always one I meter. One triangle means alone, two triangles means to connect, four triangles means gathering, and many triangles gathered means indicates a socializing atmosphere.

For the safety of people who use Augmented Reality on Site I, users can only utilise AR when walking on timber materials. The primary reason to use timber is because the timber texture is easy to illustrate the model upon. Safety was also a concern, as individuals tend to walk slower on surfaces that can become slippery, like timber. As people will be heavily engaging with mobile devices, the sounds associated with timber walkways, creaking and such, will alert users to oncoming pedestrian traffic. Furthermore, timber is considered as one of the main materials of Dian Architecture, which connects to a rich sense of history. 

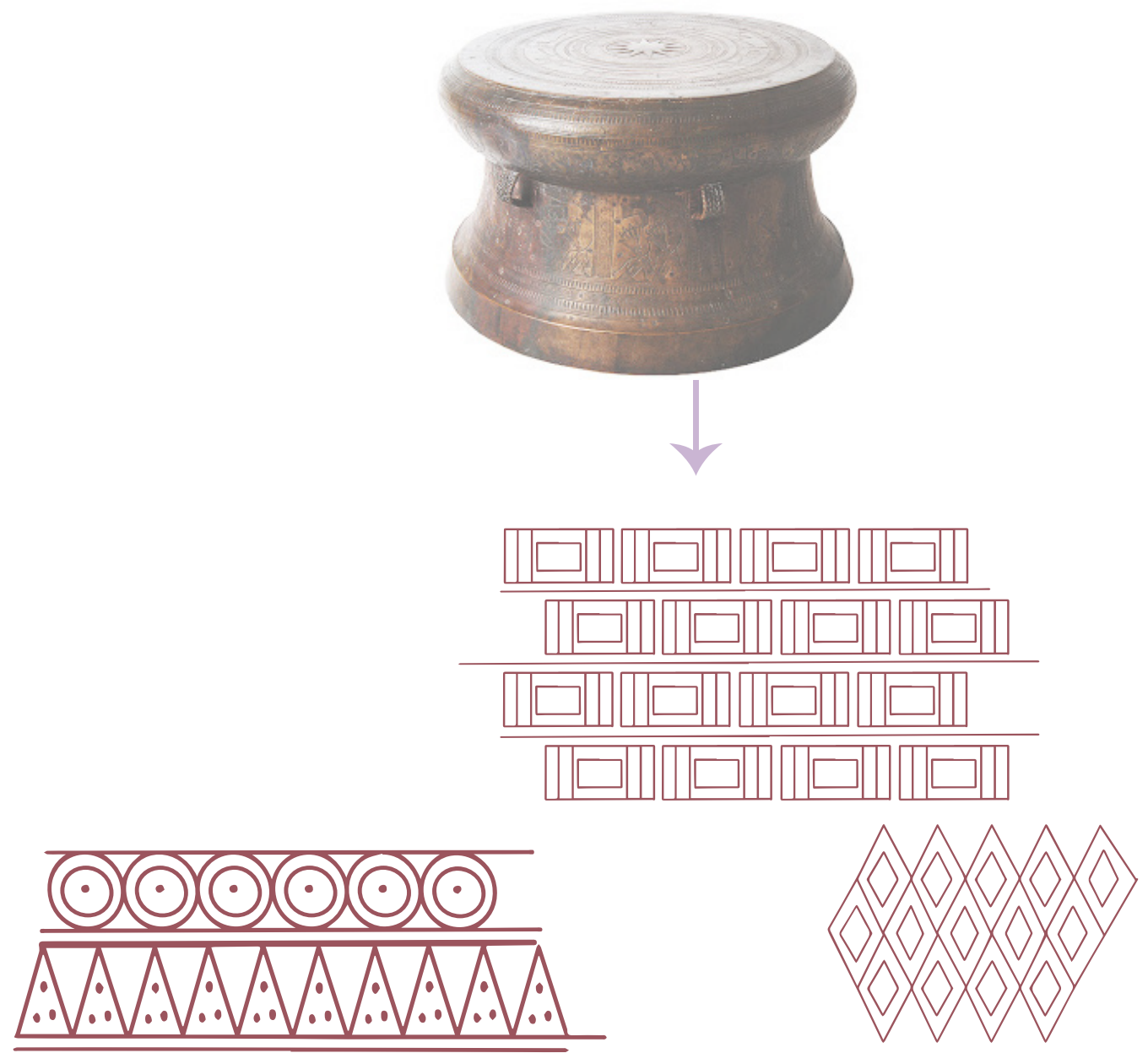

figure $\mathbf{1 8 3}$, process to get triangle
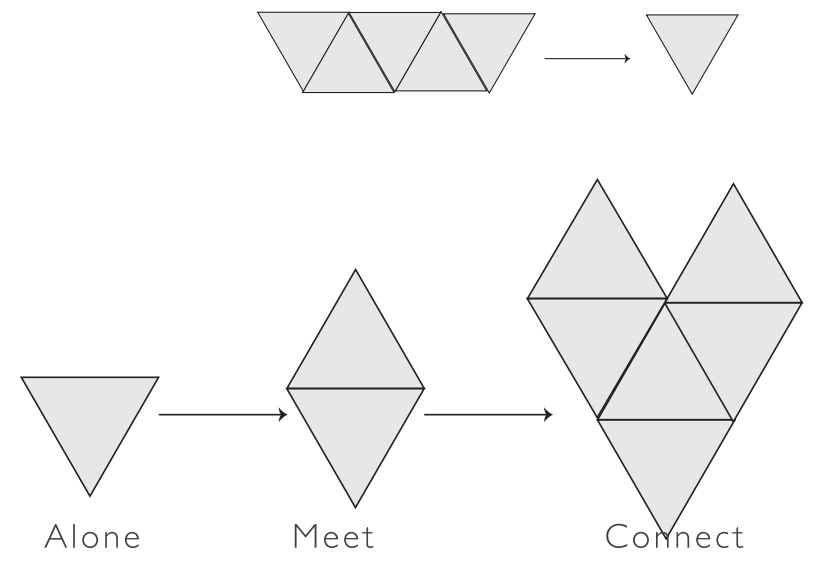


\section{I.I Forest Run}

Naming:

This zoom site is named "Forest Run" It is the entry of the park and is named as such as a connection to the story of the tiger and the $\mathrm{Ox}$.

Revealing:

It created a sense of place that is sheltered by canopy trees. limited sightlines, due to the canopy and dense underbush, are creating a tense atmosphere, as individuals cannot see where they are walking towards. The plants here are enhanced with design to create a sheltering space by using the

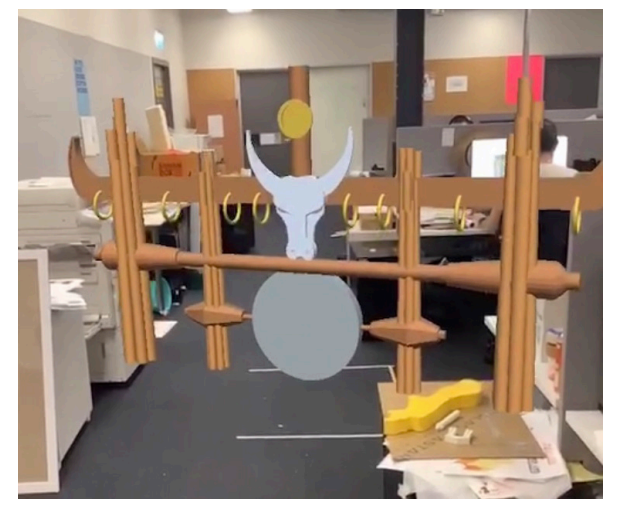

figure 184, AR models method of making a space revealing that creating suspense of the space to affect the emotion of the users. It makes people experience they are in a relatively dangerous place.
Gathering:
It created the stories of finding a safe place to hide in a forest. The AR is enhanced with the stories in zoom site I.I that illustrates the "dark" things such as Sacrifice.
In Dian Culture, people Sacrifice, and the structure of $\mathrm{Ox}$ head illustrating the wealth by using Augmented Reality. 


\section{Opening:}

The different materials differentiate where people can stay and use AR and can fully experience the physical landscape. Also, the timber pavement sizes determined how important the $A R$ is in the specific site. Larger areas encourage stopping and fully immersing in the mobile device, while smaller pathways are for travelling from one gathering space to another. The larger spaces also symbolise more importance and impact to Dian culture. Also, this allows for contrast in experience, as people who are using $A R$, will be following the timber structures and the individuals who are not, will traverse the site differently. Many of the older generation will not be inclined to follow along with the technological adances highlighted within this research project.

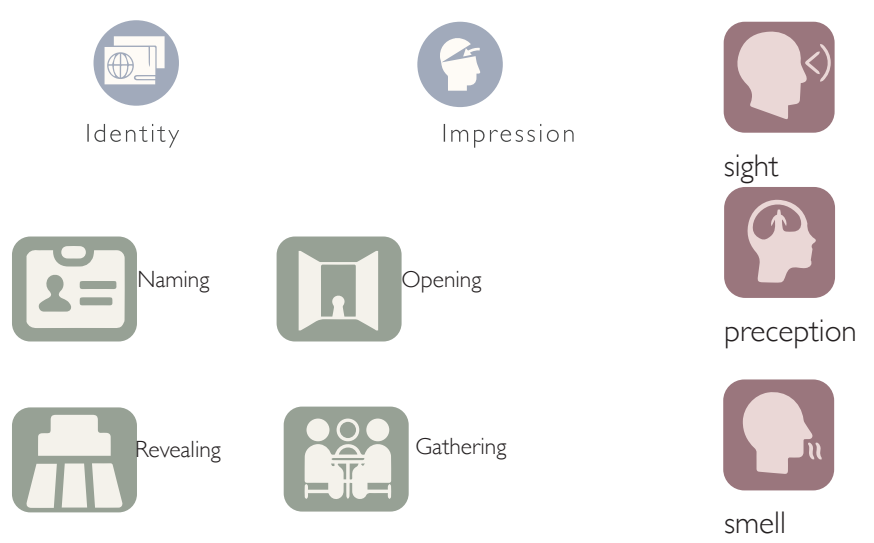





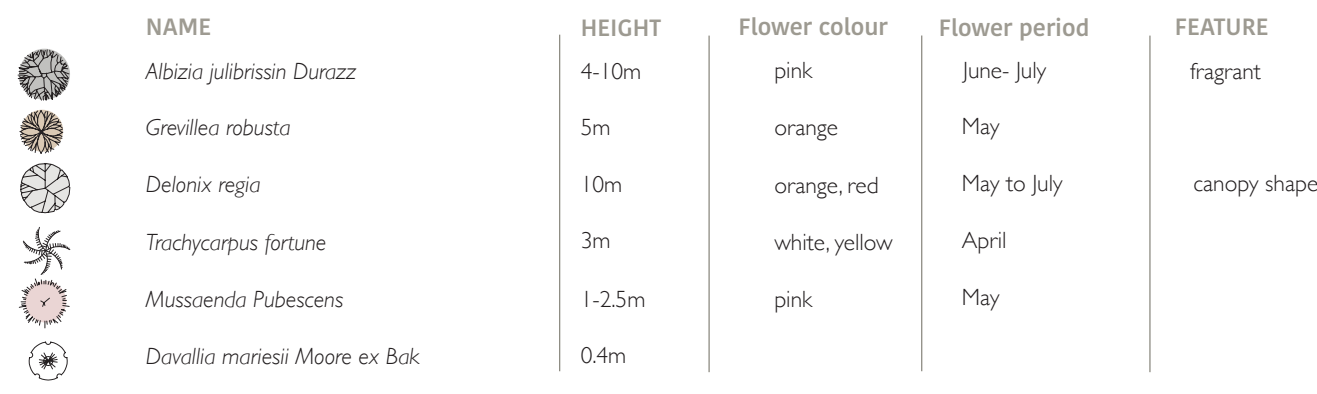

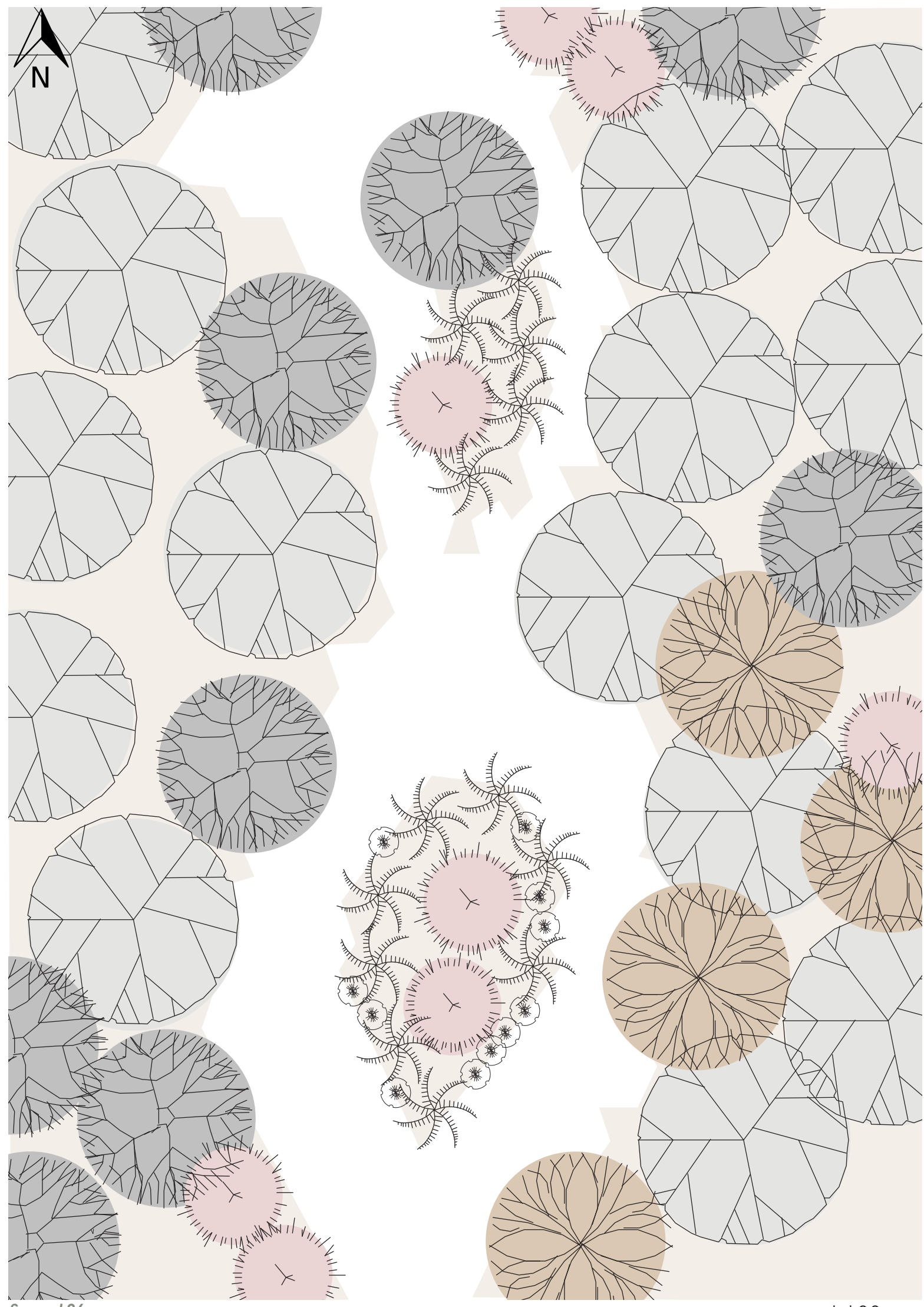




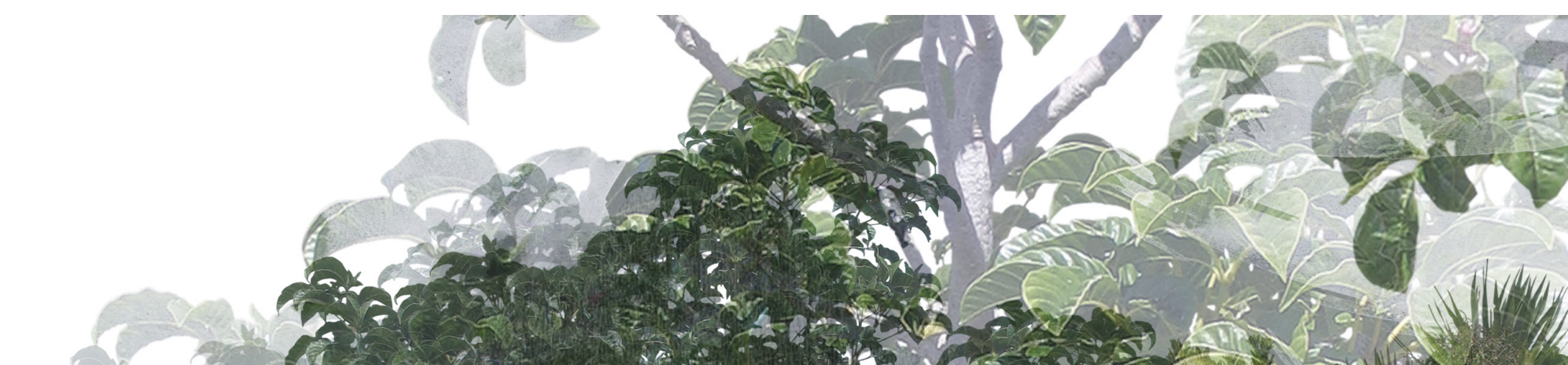
3.t.

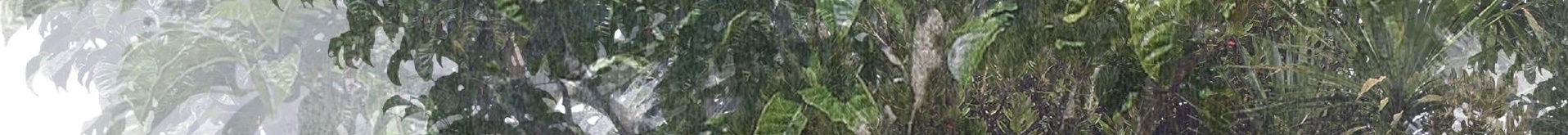

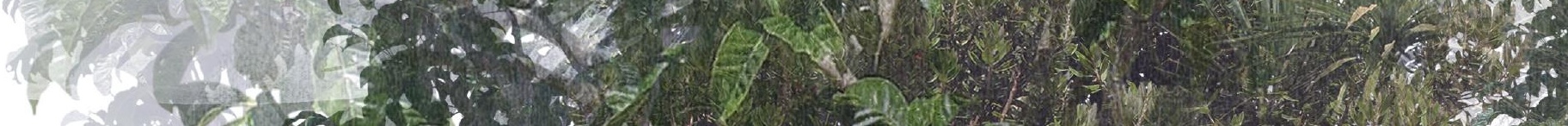

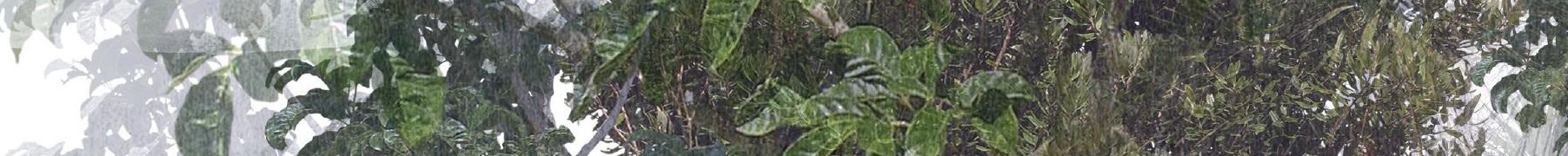
as: 3 :

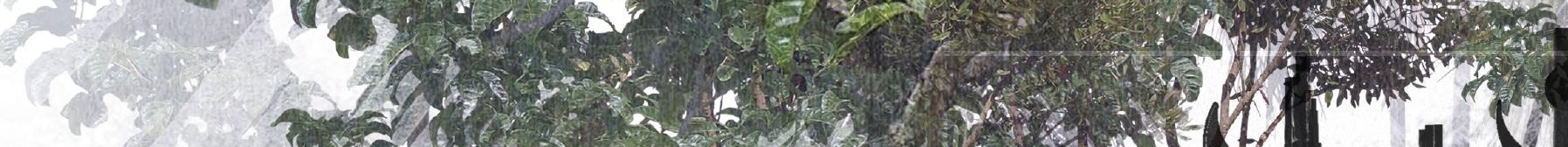

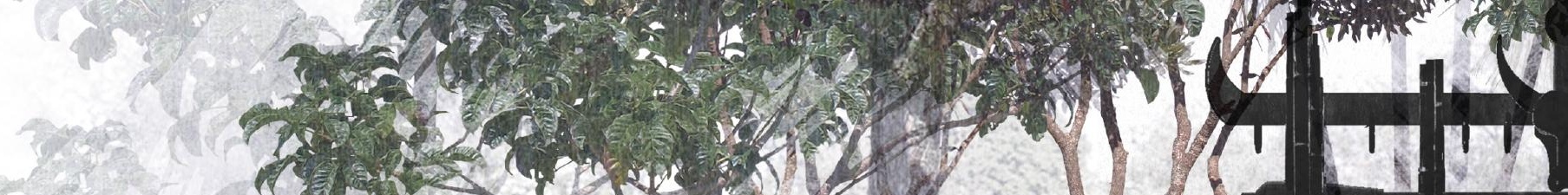
Posige $\frac{1}{4}$ 


\section{I.2 Playful sanctuary}

Naming:

This zoom is named "Playful sanctuary" and is the transition space from a journey considered to emulate danger to $t$ a journey of protection. This area relates to the bronze story where the mother $(O x)$ and child(calf) have escaped the tiger for a short while and start to feel safe again.

Revealing:

The site allows people to stay and play with installations. The shape of the canopy gets inspirations from the tool Dian people used to fish. The tool is made of bamboo, which is easy to get the material from the site. The gap of the weaving tool can adjust according to what size of fish Dian people want to catch. After the iterations by modelling the canopy structure, the final structure can reflect a beautiful shadow on the ground, and let people imagine the meaning of the pattern. Also, the playful board is to allow people to create their own Dian pattern by using a repetitive triangular panel.

figure 188, Iterations 


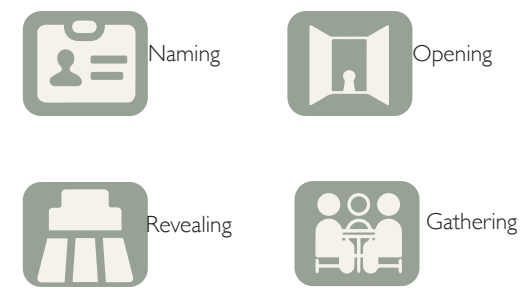

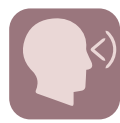

sight

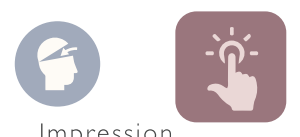

touch

Opening:

The different materials differentiate where people can stay and use $A R$, which subsequently allows them to and can fully experience the physical Landscape. Also, the timber pavement sizes determined how important the Augmented Reality is in the specific site.
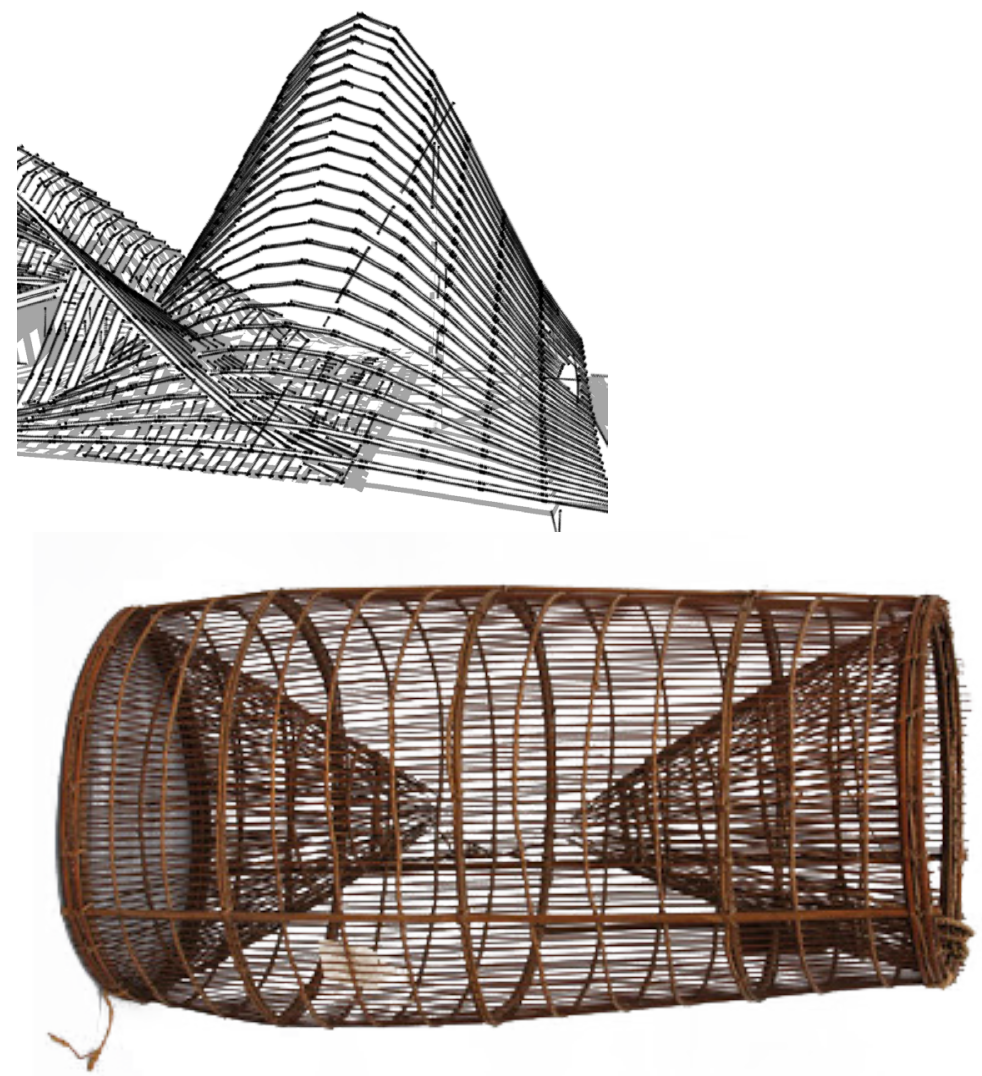

figure 189, inspiration from Dian fishing tool 


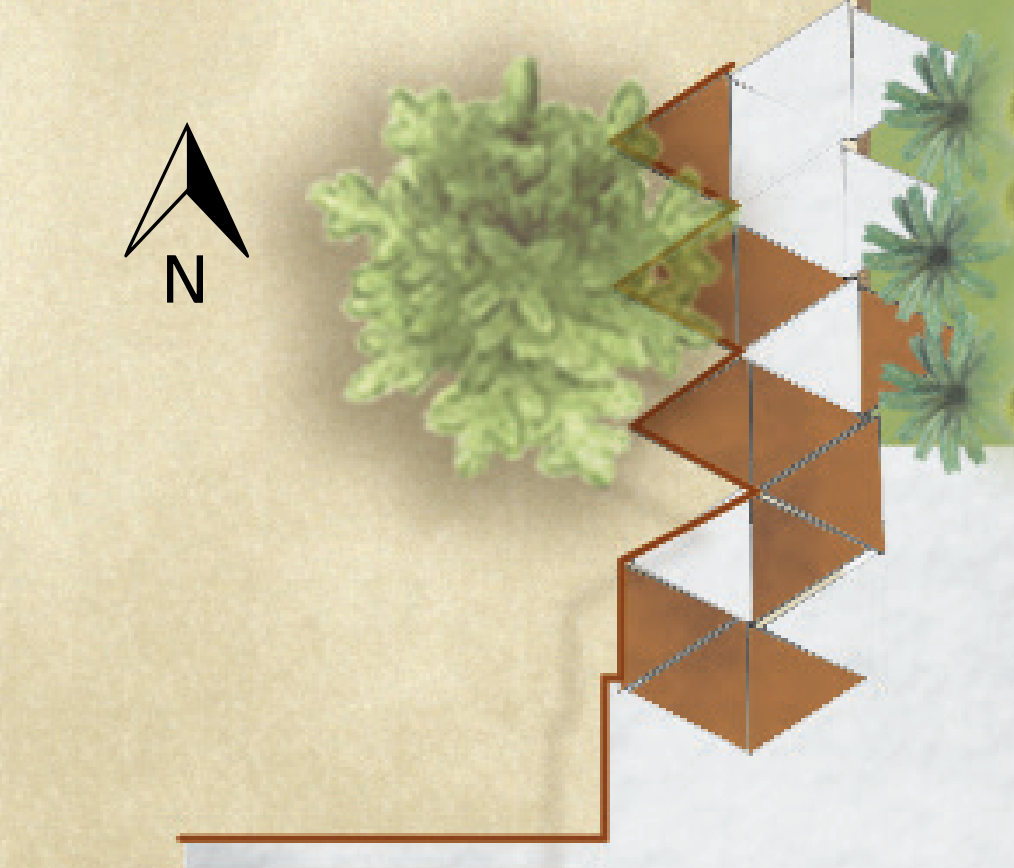




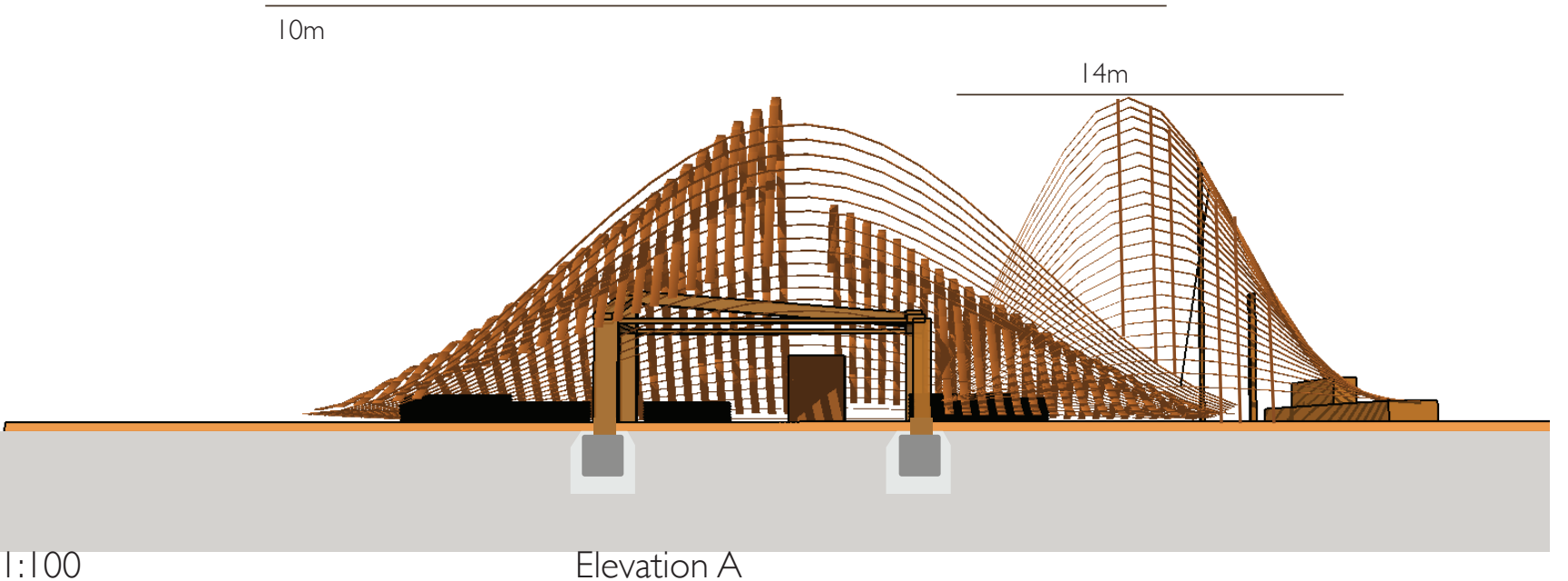

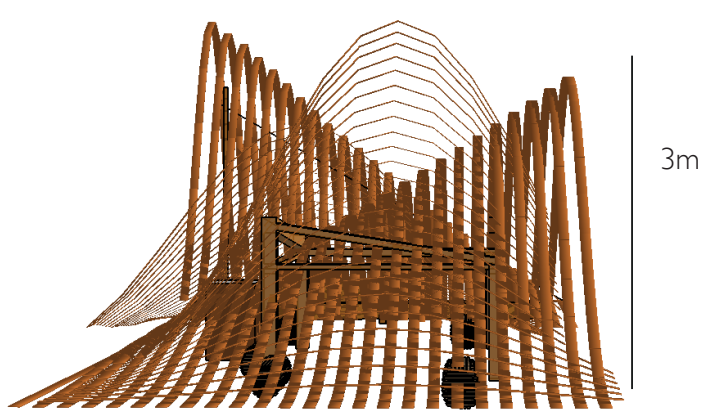

I:100 Elevation B

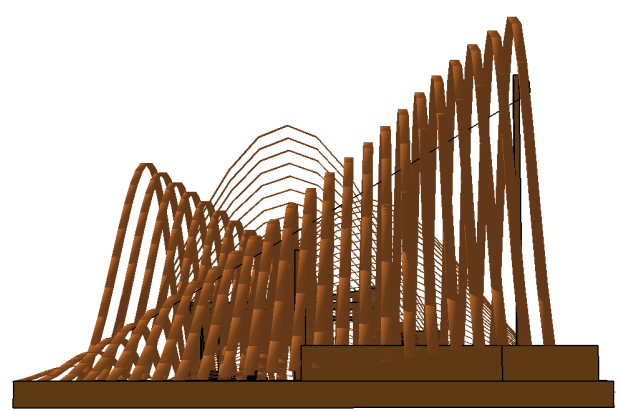

I:I00 Elevation C

figure 191. sections

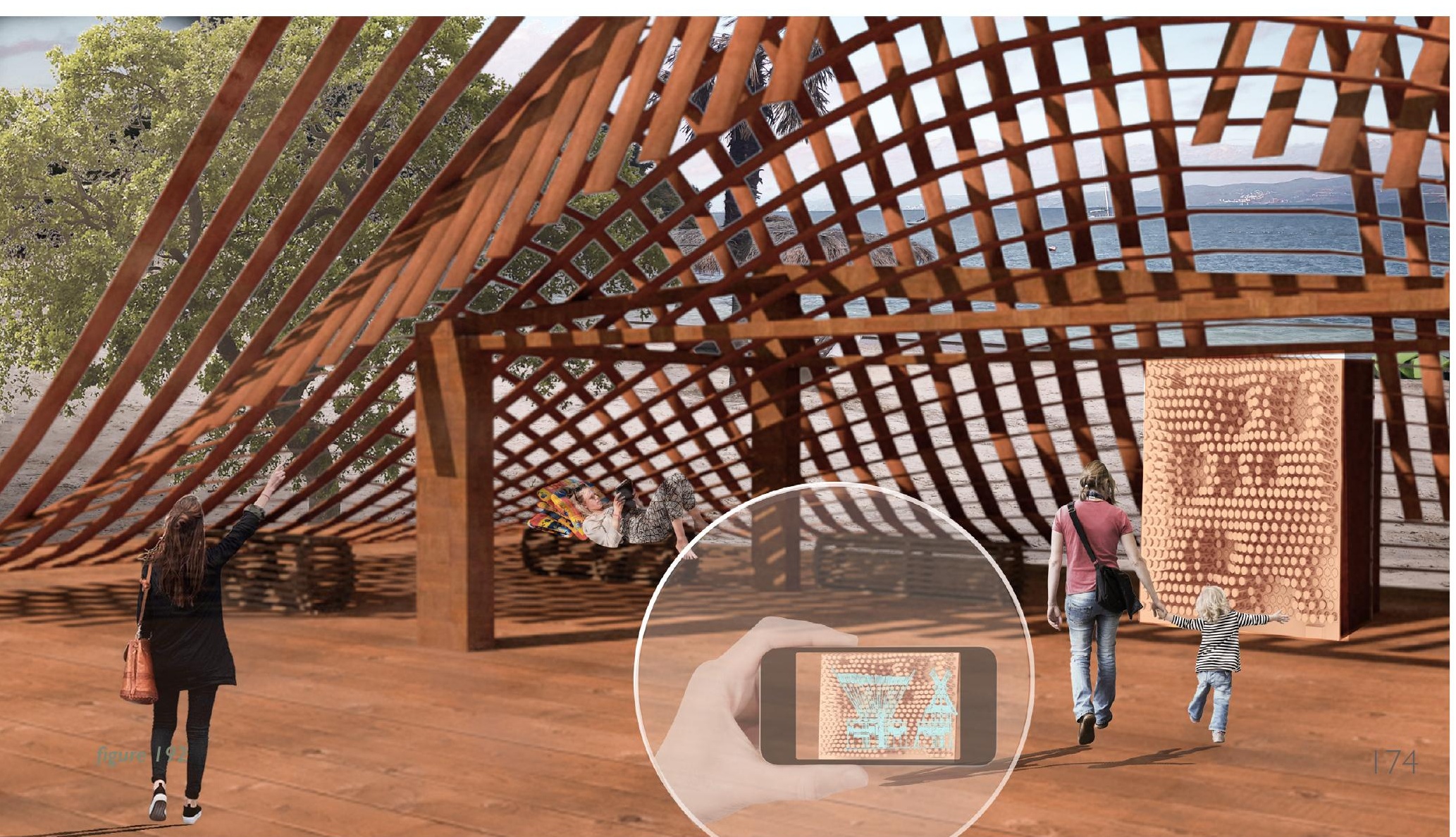




\section{I.3 the calm before the storm}

Naming:

This zoom is named "The calm before the storm" and is a large opening area with multiple uses for people. In the story of the "Ox and Lion" Bronze, the current stage in this site is the tiger attacking the $O x$, and the $O x$ is holding her calf in her arms. Because of the protection of the $O x$ and the calf's limiting views, the calf presently does not know what is happening to its mother. By treating users as the calf in the "Ox and Lion" story, they will feel calm during this period.

Revealing:

The site has multiple possibilities to allow people to do different kind of activities. It has an open view of the whole lakefront, and people can look at the mountain from the site. The revealed things on this zoom site are people will feel protected in the area. For example, the parents can look after their children as there is no visual obstruction. Also, the railing on lakefront aims to ensure the safety of both adults and children. 

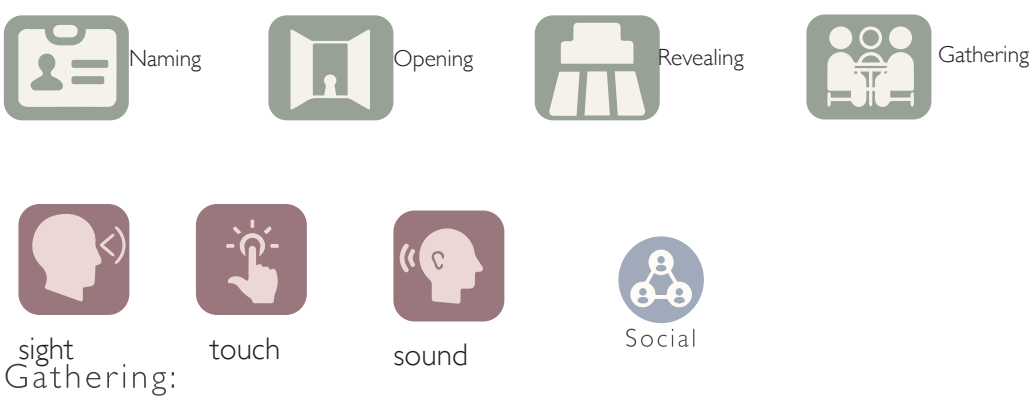

It creates a space that makes people feel like they are under protection. But as the calm before the storm, the Ox is getting weak in this stage on the site. Most people are gathered in this space, and they can play with sand, lie down on the sand, walk along the waterfront, discover the $Q R$ Code for $A R$ and explore further the Dian culture. The AR in this site will mainly illustrate Dian People's canoe, habitats and clothing. People need to find timber pavement or timber panel hidden in the sand to scan the $Q R$ code to get the Augmented Reality model. It has the opportunity to create social function for the people who are discovering the $Q R$ code and sharing what they have found through AR layers.

Opening:

It also differentiates the materials that where people can use Augmented Reality like other zoom-in sites. People can use Augmented Reality on the waterfront with Timber Pavement, or the sandy area can find timber panel. 
A

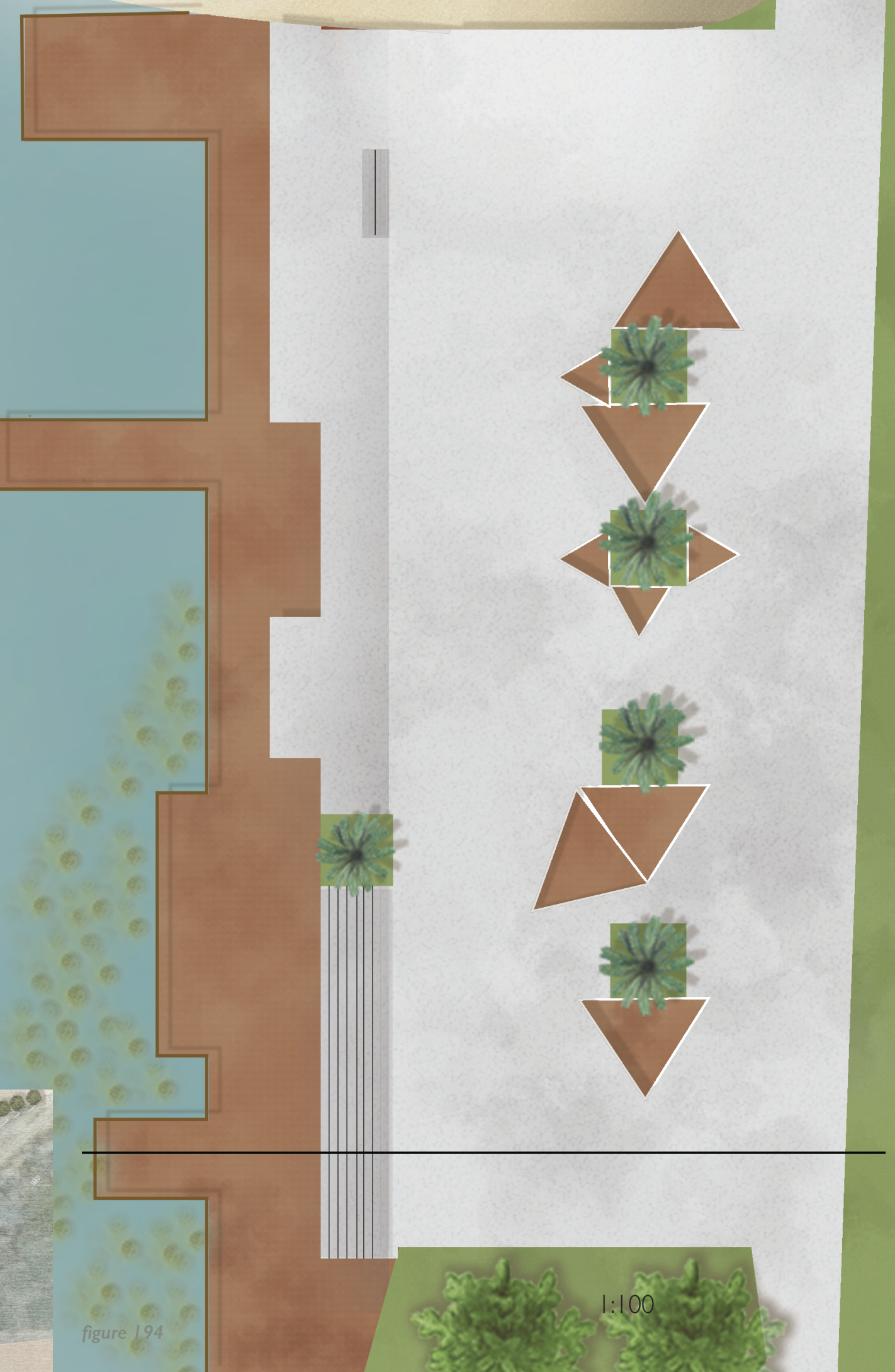




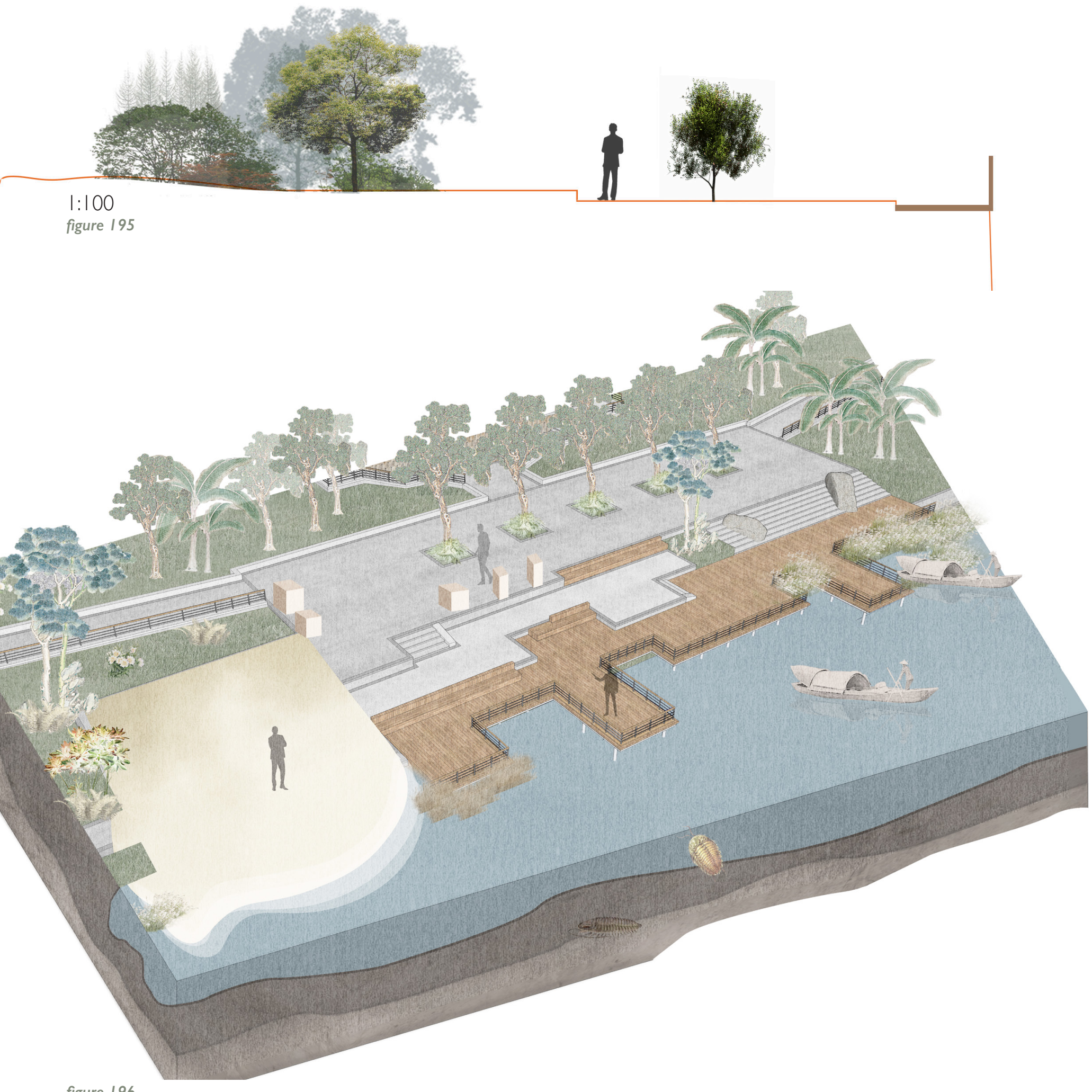

figure 196 
SITE 2

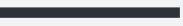


The detailed design illustrates it follows the design criteria, and will introduce different narrative methods.

The inspiration of the park is from traditional Chinese gardens in 1800 years ago. During that period, most of the gardens in China were mainly for hunting. So, the park would be like a hunting park that people can hunt the knowledge through Augmented Reality.

The main materials for site two includes gravel, marble and timber. Almost all of the chosen plants for site 2, follow the concept of confucianism in Chinese Garden, where plants represents the personality of people. It will be illustrated through plan, sections and perspectives.

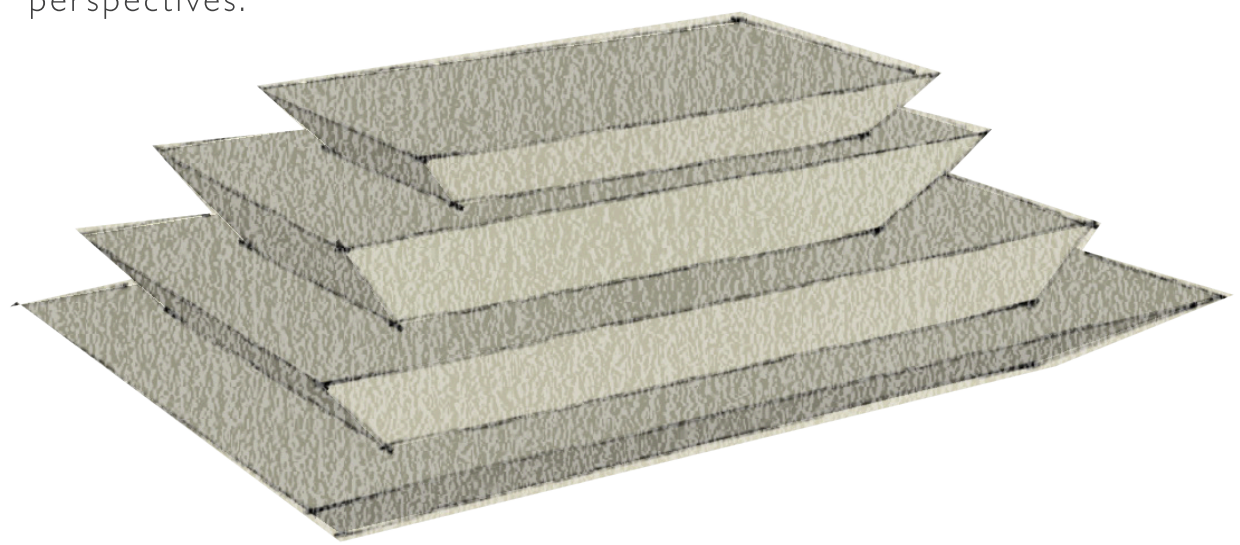

figure 197 
Site 2.1

This site is a site that uses a limited space to create a long journey for people. The journey of the site will make people feel like they are walking on the underwater remains. The function of the underwater structure is for sacrifice, with a pyramid shape. The design makes people feel like walking in a maze, and then have a sense of despair. It has a topographic change from level 1 to level 2 in this zoom-in site.

The window on the wall is a tool to collect the most beautiful view around the site(figure 190). Also, the shape of the big trees would change over time, that will turn red during spring.

There are several viewpoints like windows or structure to attract people to stay and have a look. Then they will find the QR code of the Augmented Reality layers next to the view. Then they will collect the pattern seen from the underwater remains through the AR.
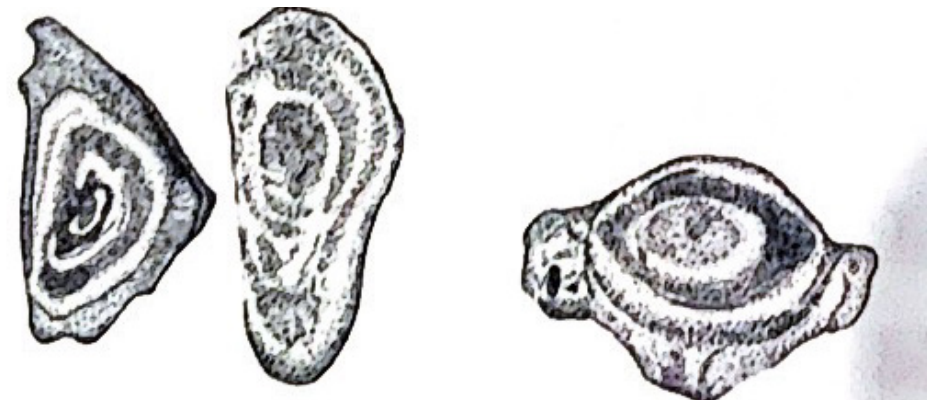

figure 198, pattern of the stones found underwater 


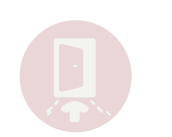

penetration

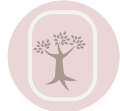

reveal

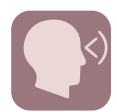

sight

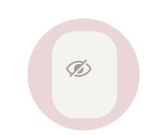

hidden

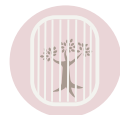

shallow reveal

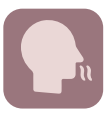

smell

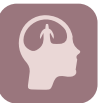

shallow hidden

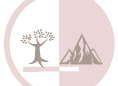

suddenly change a sene

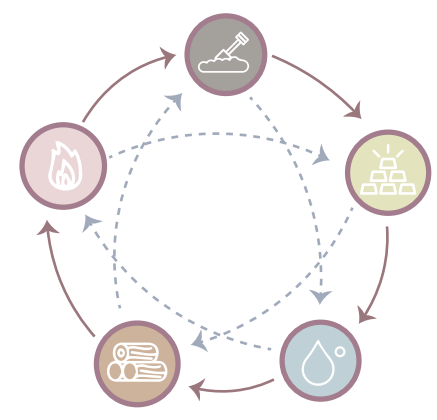

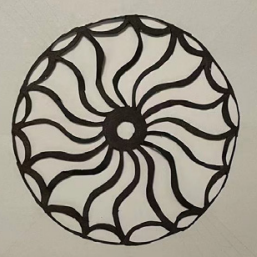
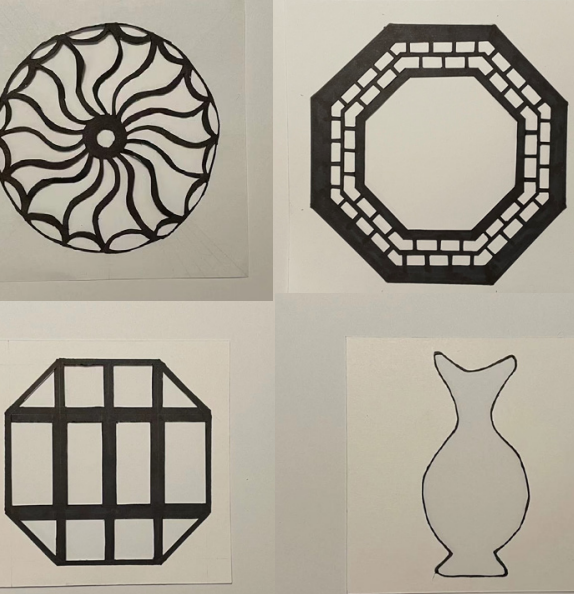
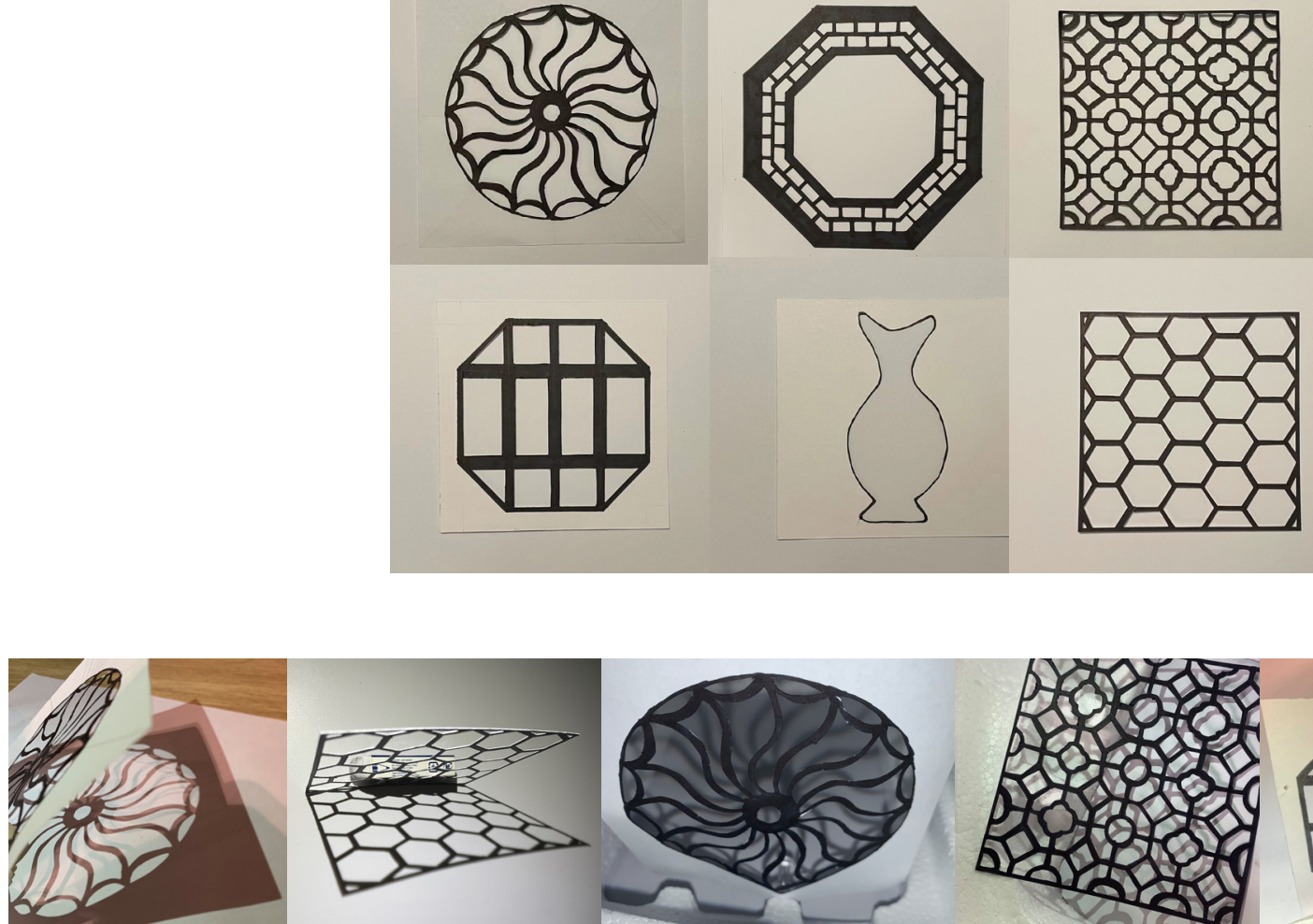

䢖

figure 199, Iterations of the shape of the window 


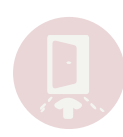

penetration

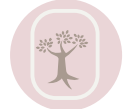

reveal

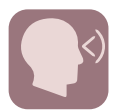

sight
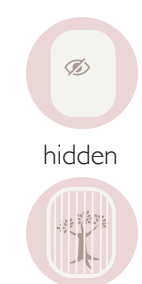

shallow reveal

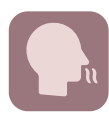

smell

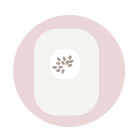

shallow hidden

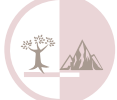

suddenly change a sene

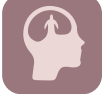

preception
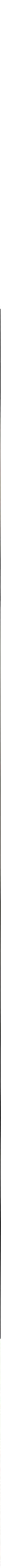


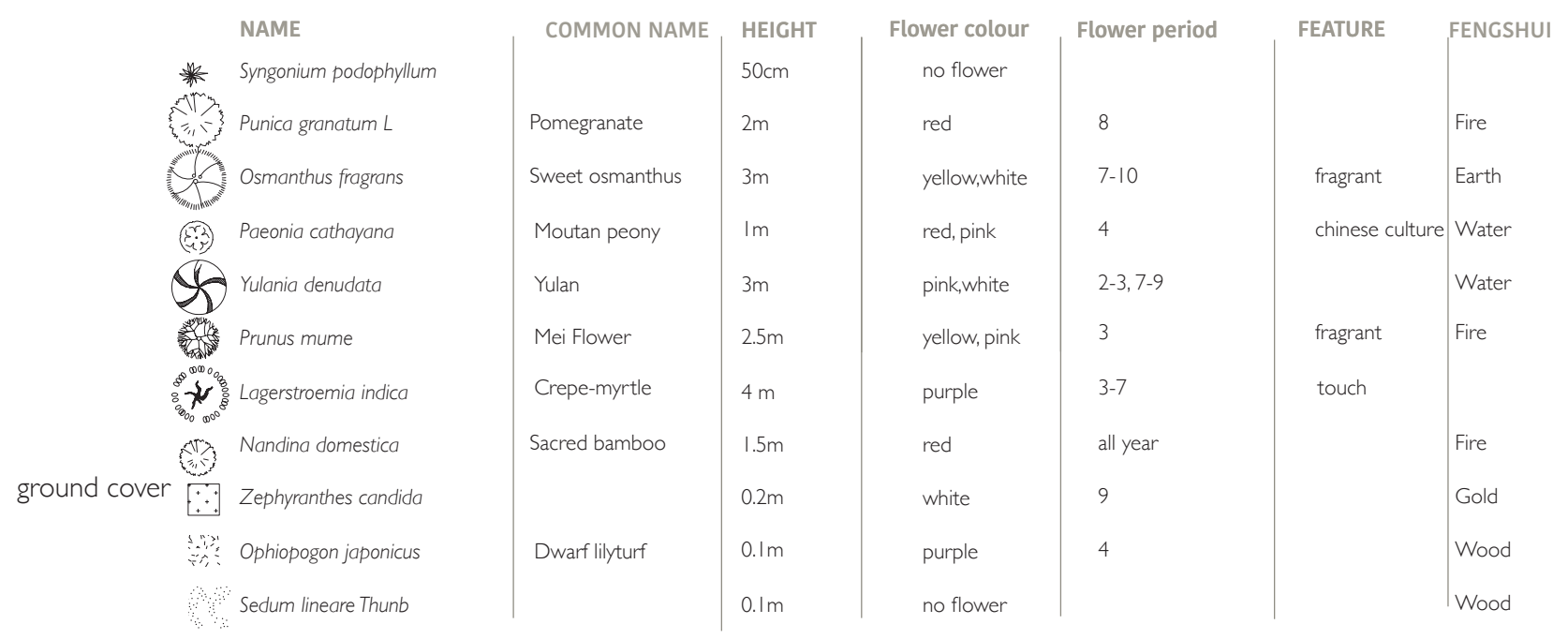

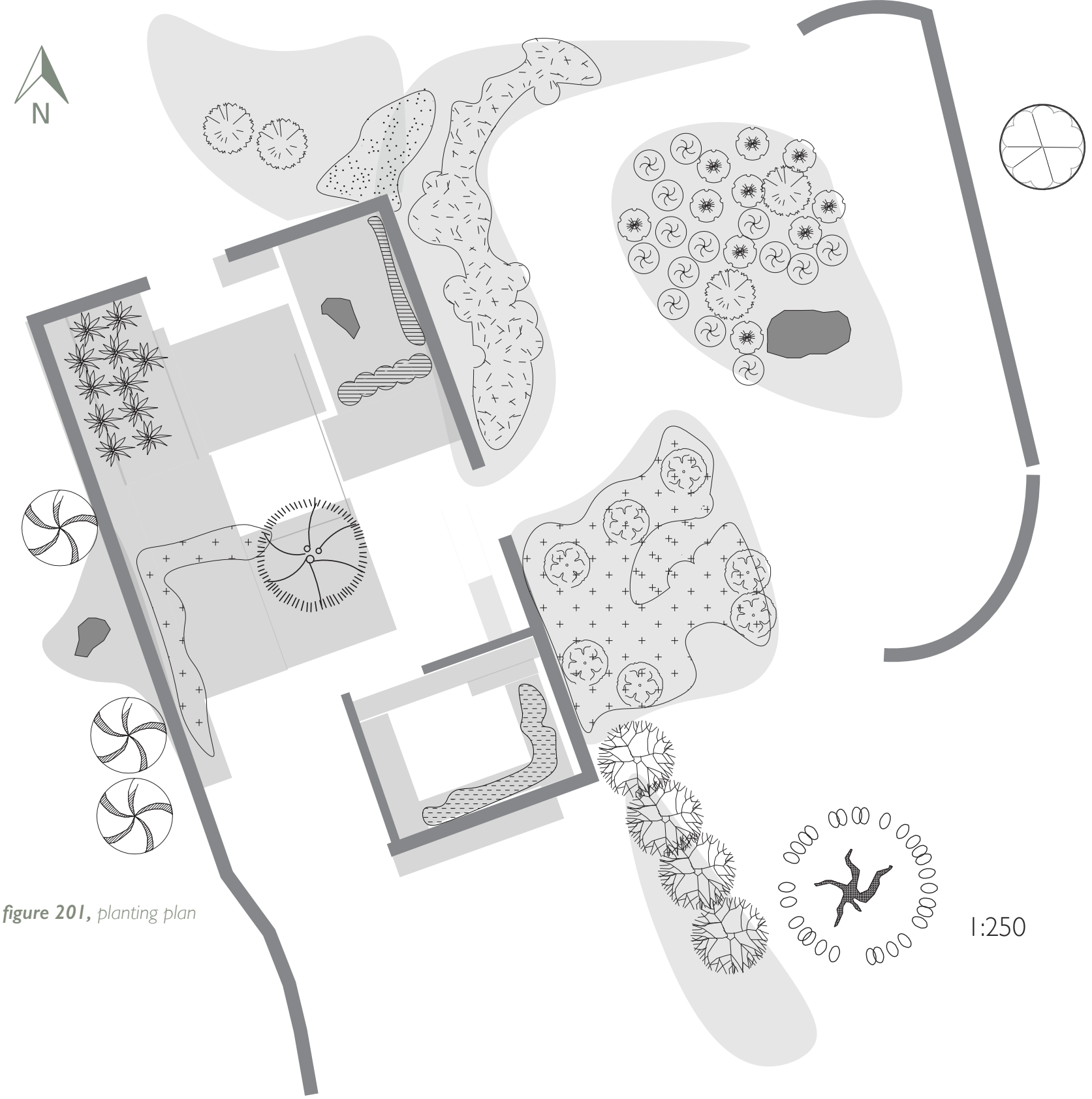




\section{2}

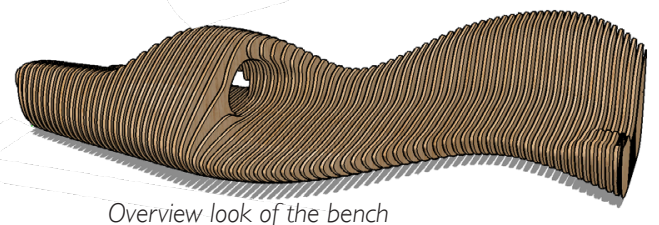

Overview look of the bench
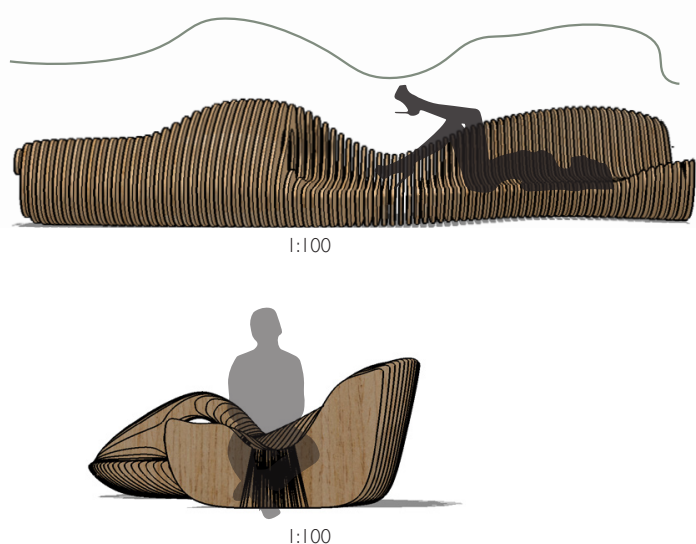

figure 202, sections of bench

Mist Machine

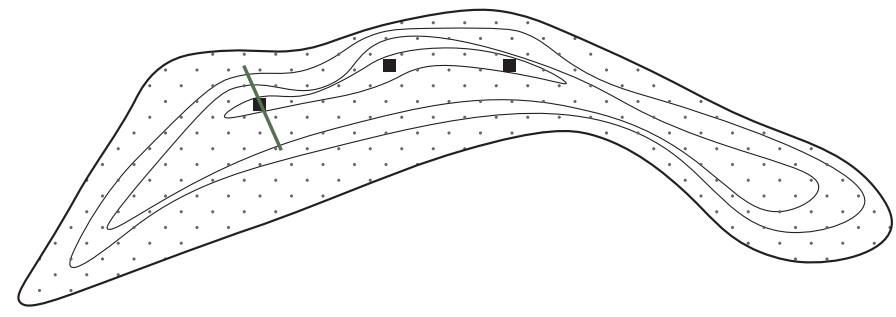

1:100

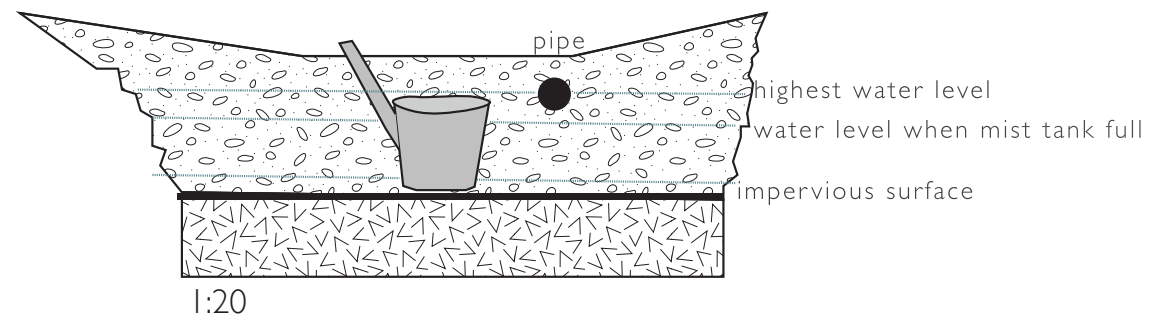

figure 203, mist diagram

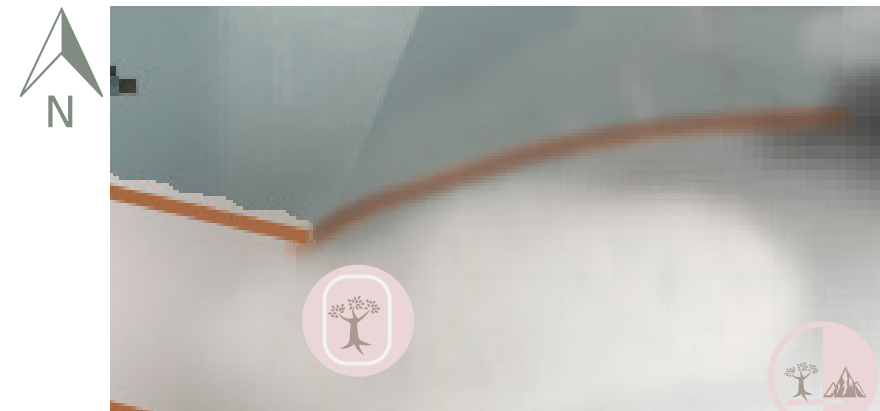

(x)

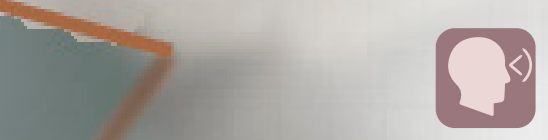

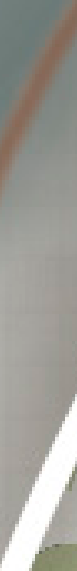

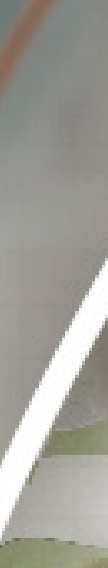
4

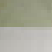




\section{3}

This zoom portrays the end of the journey. It has the concept that everything is infinite; there is no end to the trip. The shape of the bridge as developed would be the shape of an arm holding people, that consistent with the design of site 2.1. Also, the slopy fence is based on the analysis, that will make people psychologically experience they are close to water. The structure at the end of the journey is showing an infinite view. As the researcher mentioned before, it collects the views of the trees on zoom site 2.1 as the Taoism philosophy everything will back to the beginning, there is no end with nature.

As the Augmented Reality on the site, people will find that each timber column on the bridge structure has a QR code of Augmented Reality model. Then they will found the model of the design of the underwater remains. 


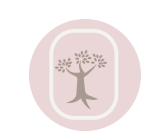

reveal

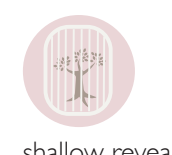

shallow reveal

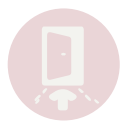

penetration

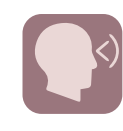

sight

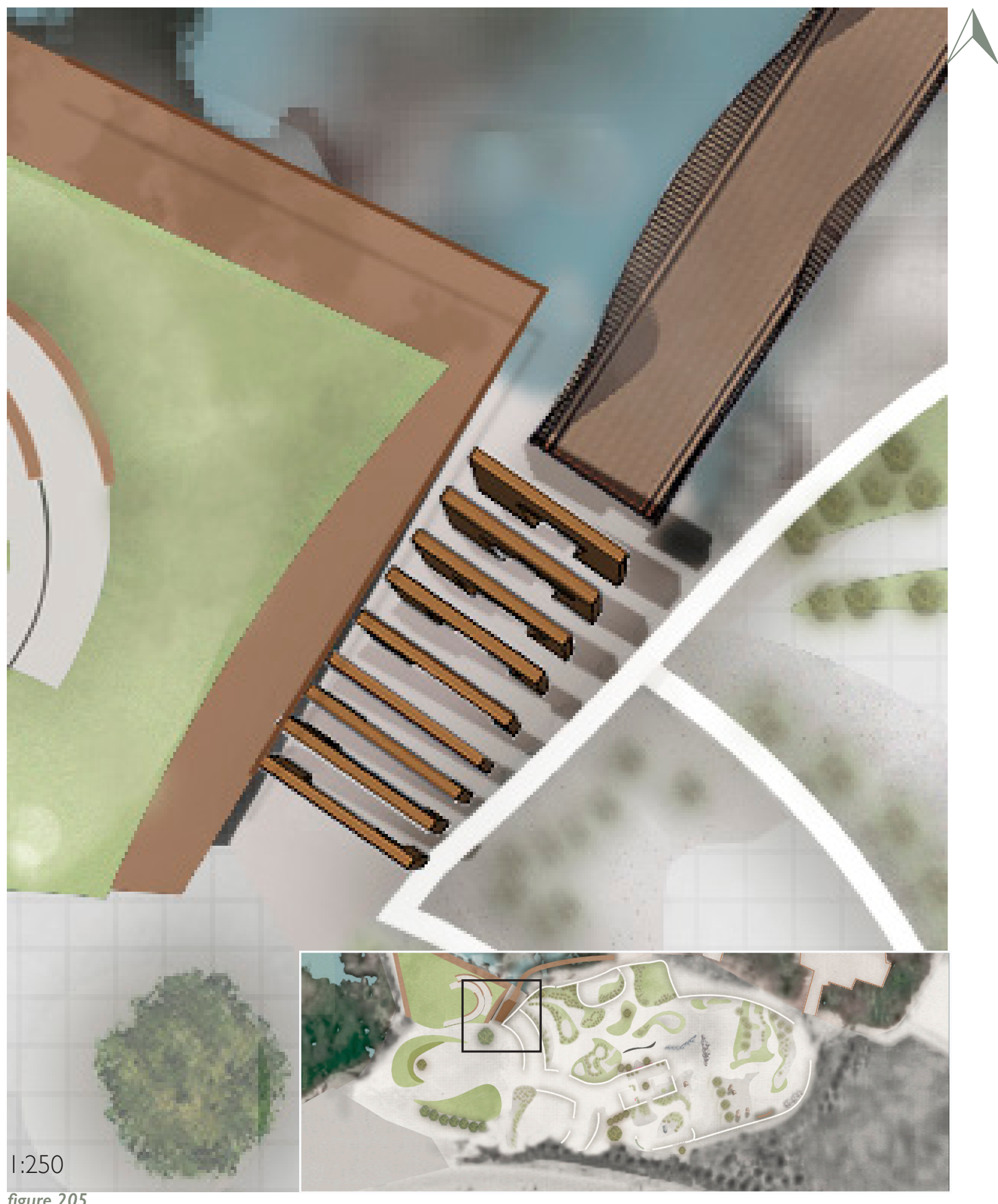



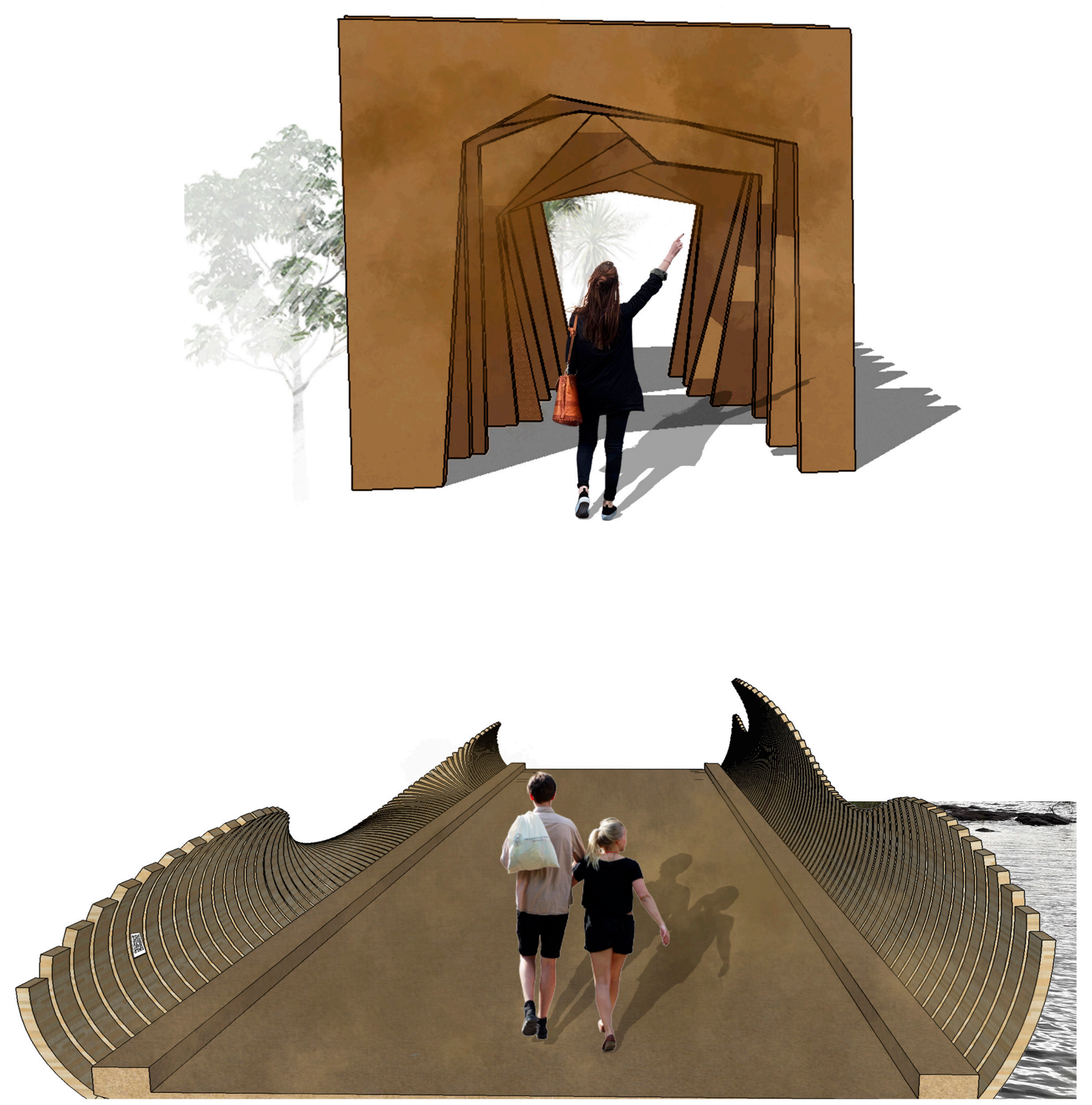

figure 206, perspectives of infinite structure and bridge 
Spring

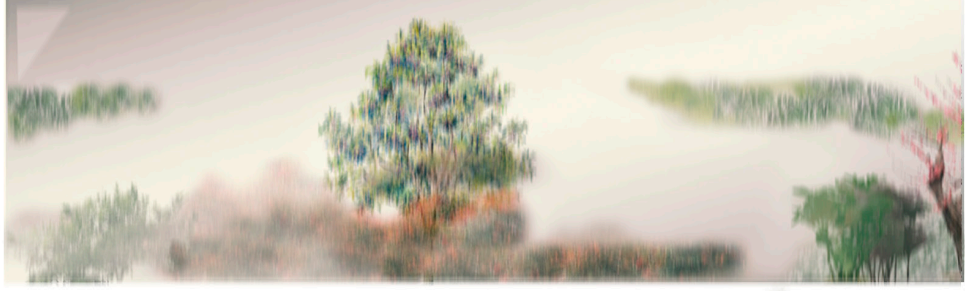

Autumn

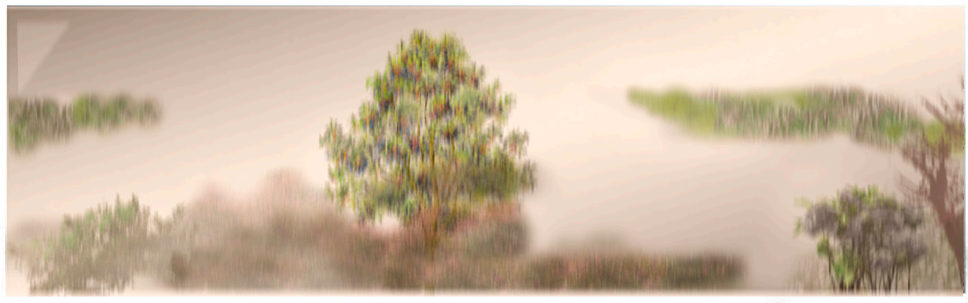

Summer

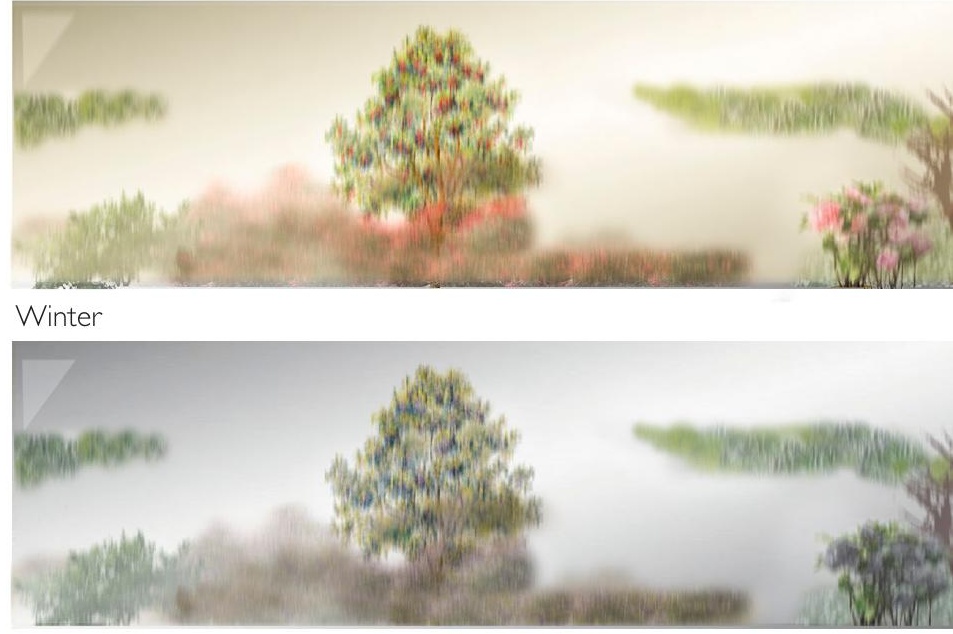

\section{ㄱ.}

figure 207, Change over time during a year.

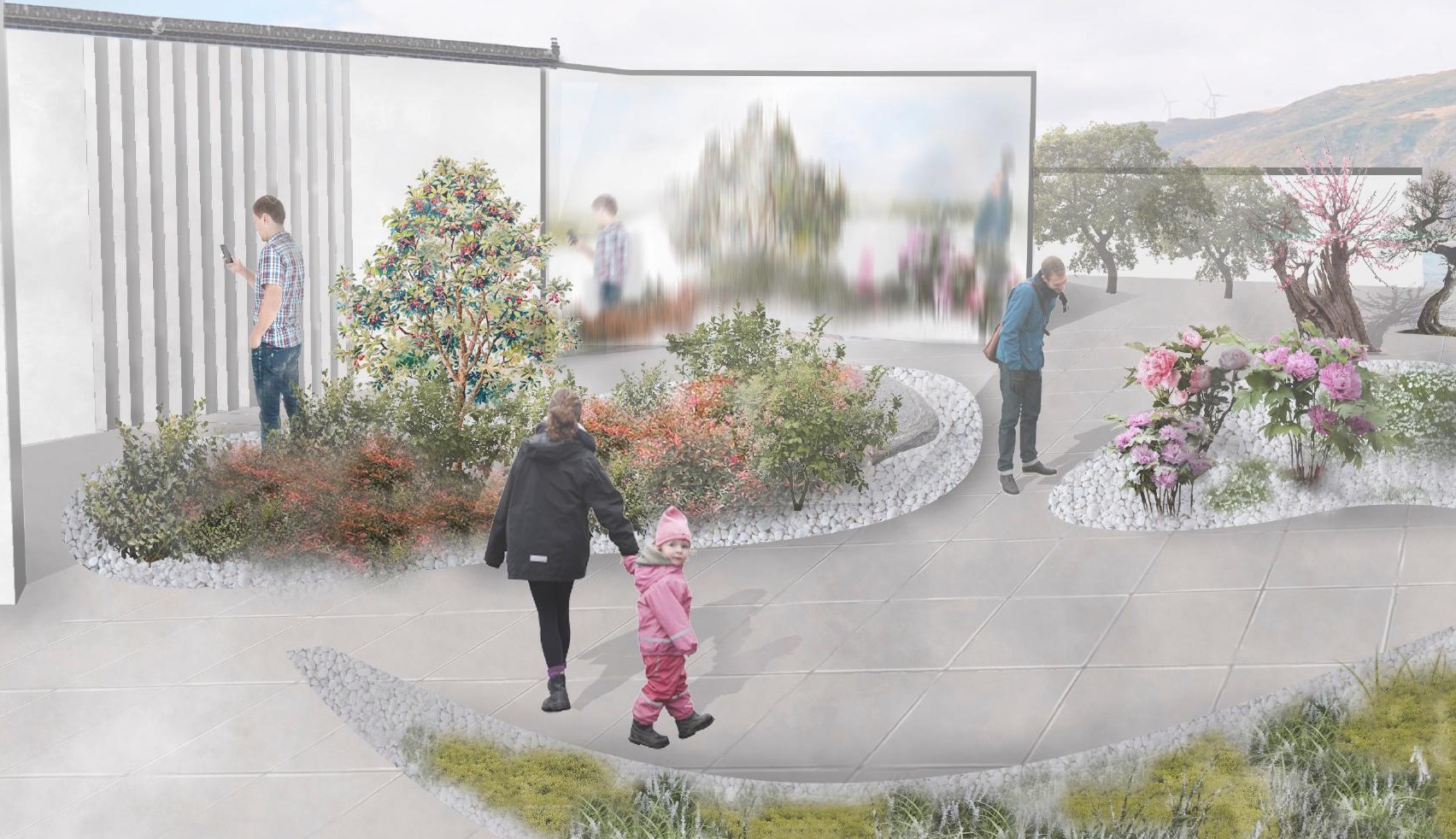


CONCLUSION

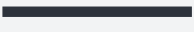




\section{RELECTION}

The initial research question asked, 'How to use Augmented Reality to reveal the hidden history of Lake Fuxian and enable the design of existing landform through site narratives?'. This research uses this the physical Landscape and Augmented Reality to contribute to a deeper understanding of Lake Fuxian and the narratives that link it with its cultureal history. The methodology allowed an idiosyncratic clarification of cultural and historical values to be applied to site discovery and development. It explored the disappeared history and culture of Lake Fuxian in a futuristic and inventive way.

The Literature Review, permitted the identification of strategies to enhance the design and highlight Lake Fuxian. In this part, it revealed three key elements

- How Chinese philosophy affects the design of a Chinese garden,
- How to design a narrative Landscape and finally;

- How to use new technology to enhance narrative Landscape.

After that, the case studies investigated how can landscape design be enhanced with Augmented Reality, Chinese narrative gardens and Waterfront design work in sync. Both of the studies took a broad sweep of relevant cultural values, identity, narrative and new technology. However, a further connection of landscape narrative and the hybrid space created by both the physical landscape and AR technology need to be investigated. Also, the data of specific users for the application needs to be collected and more targeted for the predicted participants. 
This approach provides a large-scale scope to a closer theoretical human-scale analysis through site exploration. This data aided in the search for two sites to further develop at a detailed scale. This data enabled a unique research methodology through its topic, approach, and solution.

To design an engaging landscape narrative that is enhanced with new technology is a challenging task. Augmented Reality was only invented no more than 30 years ago. It is also an application without much connection to Landscape Architecture, where methodologies of use had to be developed specifically for this topic. Furthermore, Augmented Reality's content has a digital interaction and visual connection with the physical landscape, which is only possible with advanced knowledge of landforms and historical narratives of the site.
The two sites heavily rely on the framework because the culture 1800 years ago, was managed by the Chinese government. This meant that all designs were influenced by the concept and rules of Fengshui. The overall experience of the design is to create a sense of place through both the physical design and the Augmented Reality model, where individuals are able to explore the history and culture of that space. Furthermore, the design conveys unique ideas in different areas by utilising various design criteria's and implementation strategies. 
The design outcome and how it acted to answer the research question, was based on the literal sense of narratives and storytelling and how the representation of cultural and historical values was understood. For instance, one of the detailed designs in illustrated abstract forms of the tool Dian people used for fishing on physical space. However, when the AR layer is activated it shows how the tool looks like by. More importantly, it relies on the understanding of the experience with hybrid space.

The ability to freely explore the locations through both physical and digital movements allows for a cohesive understanding of past and present. Furthermore, the physical landscape becomes a tool for AR technology, as they are so closely tied together. Cues from the landscape inform people that there is more information to be gathered and understood. 
Overall, the design-led research and final design outcome were able to answer the research question "How to use Augmented Reality to reveal the hidden history of Lake Fuxian and enable the design of existing landform through site narratives?" . After designing the physical landscape, the physical landscape brings users an abstract feeling of the site, and the feelings attract people to discover the hidden stories of the site. Then, the Augmented Reality illustrates the concrete aspect of the Dian culture, and the history of Lake Fuxian. To conclude the content showed from Augmented Reality, illustrates important features, such as the underwater remains, sacrifice, the rituals, Dian architecture, and etc. which are hard to put or rebuild on the physical landscape. Furthermore, the methodology and framework drive the research to an outcome on Lake Fuxian. 


\section{LIMITATIONS}

Going through this research, there were some limitations to the study and the design, particularly changes to the methodology process.

At first, at the start of the research in late March, and during that time, the researcher has chosen Lake Fuxian. Then, the pandemic of Covid-19 in New Zealand and the subsequent lockdown has forced every student to work from home. During the lockdown period, it made everyone change the way interacted with work. A change of site was considered after May, as it was not easy to visit the site from New Zealand. However, careful consideration and because of the information found on the hidden narratives of the site, Lake Fuxian was considered the most appropriate for this research.

The Covid-19 pandemic limited the way site analysis was conducted. Instead of physically visiting the site and experiencing firsthand the conditions of Lake Fuxian, reliance on third parties was needed. Video conferencing and interviews with relatives that live close to the site was the only way to gain this information from long distance. Additionally, due to the distance, accessability to essential data like contours, pollution levels and topology was difficult to obtain as it is not commonly available to the public. More importantly, the aspect of the research was not able to be conducted, such as observation of human interaction and movement. The human scale is hugely important and critical to design iterations, so assumptions were made based on previous visits to the site. 
If there was more time, the research could have explore the design of additional sites around the lake, by following a historic timeline. This could be suitable for people that would like to contrast the history and culture based on different periods.

If this research continued, or was able to be conducted with a team of designers, a downloadable app would have been created and uploaded alongside this document. However, another simple tool (Aug In and Layers) was enabled to show the Augmented Reality model from the perspective of mobile devices. And the potential users will follow the functions demand:

a. Location based - would be easier to show Augmented Reality automatically when the user goes to a specific space.

b. Playful - have a function that allows people to draw things from their phone and and add to their understanding of the site.

c. Social - have the function for people who want to add friends, then the design will be a tool to educate groups, or perhaps classes of students simultaneously.

d. Change over time - where Augmented Reality content can change over time, which will attract people to explore the app many times 
Overall, the research did an excellent job in representing the landscape narrative with Augmented Reality perspective. Although changes to the world over last 12 months limited the reach and detail of this project, the final design perfectly captures an idea that can be developed and grown when more information and time is made available.

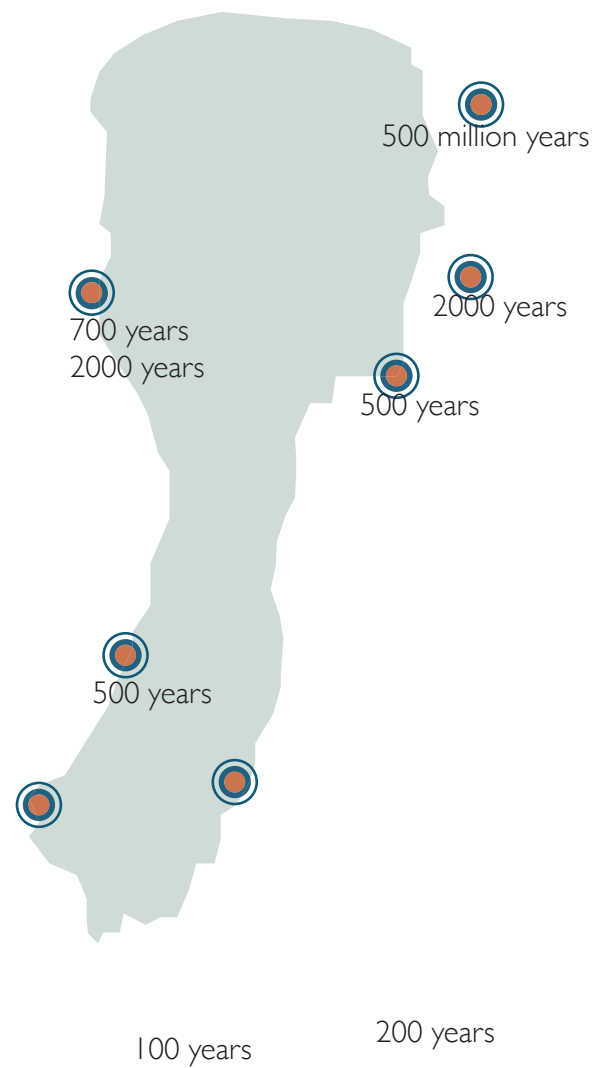

figure 208, Journey follows a timeline 


\section{CONCLUSION}

From an international perspective with many roots in Chinese culture, it is difficult to comphrehend cultural identity from afar. What makes one small town different from all of the other areas of China? How would foreigners identify individuality and uniqueness in site when locals could not do the same? When applying this to Lake Fuxian specifically, it is difficult to understand the past of the lake when there were no physical and visual cues to the rich history of the Dian culture. It was when that the question arose-what can a landscape architect do to relink the landscape to its history?

When trying to finalise which history and culture are essential to represent through Landscape Design, there are some issues with the demonstration of the past. For instance, it will cost a lot to rebuild the architecture of Dian, particularly the underwater remains. Although there is enough money to build theses unique things, could be considered a waste of money as no one can live in those buildings. Using digital means is a less permanent solution but it does not make it more affordable. Virtual Reality needs to use specific equipment that is not convenient to move. Conveniently, Augmented Reality can be used by a smart device such as a mobile phone. When people use Augmented Reality, they are still engaging in the physical landscape. Therefore, Augmented Reality is a good tool as it limits re-building costs and space, as well as still placing importance on the physical landscape. 
As a Landscape Architect, it is crucial to know how to design a narrative Landscape in different ways. The narrative strategy of a Landscape design should follow the ways of how locals used to design and tell a story. The way traditional Chinese gardens tell and explore stories is extraordinary and different from other countries. In this research, used narrative strategies were drawn from traditional Chinese gardens and Chinese philosophy. However, the uniqueness was emphasised through Dian culture and values.
The research also touches on the issues of how Augmented Reality enhanced the physical landscape to create an abstract atmosphere for people. Overall, the Augmented Reality and physical Landscape connect to create a "hybrid space" that became an engaging and excellent way to tell stories through a visual platoform. 
The research delivers an understanding of the forgotten history and highlights the identity of site. Looking back to New Zealand, as a bicultural country, there are a lot of hidden stories in the land. The same research can be used in a New Zealand setting to represent the hidden narratives of the space. Designers could follow the same methodology that was presented in this document to bring the values and narratives from both Māori and non-Māori in an AR fusion.

Looking into the future, it is a sound prediction to have the opinion of Augmented Reality or other new technologies to play a huge role in the development of Landscape Architecture in our daily life. When it becomes more and more popular, Augmented Reality will expand and develop, reaching all demographics and skill levels throughout the world. People will be experiencing both the physical world and virtual layers on top of one another, making the knowledge and understanding of our world the best it can be. When this technology is at a stage that is widely utilised, then, everyone can be a designer of the physical space. 
BIBLIOGRAPHY

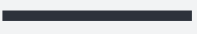




\section{REFERENCES}

Alexander, B. (2017). The New Digital Storytelling : Creating Narratives with New Media. ABC-CLIO, LLC.

Borgeat, F. (2016, Sepetmber 30). Transforming Experience: The Potential of Augmented Reality and Virtual Reality for Enhancing Personal and Clinical Change. Retrieved from Frontiers, public health: https://www.frontiersin.org/articles/10.3389/fpsyt.2016.00164/full

Chan, W. (2008). A sourse of book in Chinese phylosophy. Princeton: Princeton University Press.

Chen, A. (2015, December 7). Features of Lake Fuxian . Retrieved from Baidu Wenku: https:// wenku.baidu.com/view/425dc6df168884868762d6ee.html

China water quality standard. (2018). Retrieved from China Jishengwei : http://www.nhfpc.gov.cn/ zwgkzt/pgw/201212/33644.shtml

Fuxian Climate. (2020, February 13). Retrieved from Climate Data: https://en.climate-data.org/ asia/china/yunnan/fuxian-564473/

Gobster, R. E. (2019). The Nature and Ecology of Aesthetic Experiences in the Landscape. Landscape Journal, 38.

Grigore C. Burdea, P. C. (2003). Virtual Reality Technology. New Jesey: Jogh Wiley \& Sons.

Hillman, B. (2003). Paradise Under Construction: Minorities, Myths and Modernity in Northwest Yunnan. Yunnan: Asian Ethnicity

HZ, C. Y. (2008). Macrozoobenthic community of Fuxian Lake, the deepest lake of southwest China. Ecology and Management of Inland Waters, 116-125.

J. M. Zheng, K. W. (1998). Virtual reality. IEEE Potentials, 20-23.

Jones, M. (2003). The Concept of Cultural Landscape: Discourse and Narratives. Landscape Interfaces.

Kaul, V. (2014). Globalisation and crisis of cultural identity. Sociology and Anthropology, 12-21.

KunmingNews. (2019, March 25). Housing demolition and relocation in Lake Fuxian. Retrieved from Kunming Information Net: https://m.kunming.cn/news/c/2019-03-25/12608681.shtml\#/

Yuxi News. (2017, January 6). Fuxian Lake has 7140,000 visitors in 2016. Retrieved from Xinhua News: http://zgly.xinhuanet.com/2017-01/16/c_1120321006.htm

Ou, M. (n.d.). Esperance Waterfront. Retrieved from moool: https://mooool.com/en/esperancewaterfront-by-hassell-studio.html 
Schofield, E. (2013, November 7). Top 10 PLAYscapes Competition: Professional Category. Retrieved from land8: https://land8.com/top-10-playscapes-competition-professional-category/

Schudson, M. (2009). Lives, laws, and language: Commemorative versus non $\triangle$ commemorative forms of effective public memory. In M. Schudson, The community reviews (pp. 3-17). San Diego. Qiao, P. (2014). Chinese Culture Influence Chinese garden. Retrieved from Zhejiang Forest University: https://zlxb.zafu.edu.cn/fileZJNLDXXB/journal/article/zjnldxxb/2005/1/PDF/22-1-22. pdf

Province, P. G. (2016, April 15). Introduction of Yuxi. Retrieved from People's Government of Yunnan Province Website: https://baike.baidu. com/reference/13139/2e52uUvOc_DrWTauMMQ7whKHKTjZPKdfe HIg5r7jLOAUwwj0g_1ssBAj2YFCrhikfTvxGwb4jBiEpEb4OZ31oCliljnKtGnsyee7gNWv0ihgV_ alQ8WCWMWOMQD

Richard E, C. \&. (2019). The Nature and Ecology of Aesthetic Experiences in the Landscape. Landscape Journal, 38.

Wenku. (2013, June 23). Retrieved from The design of Lion Grove Garden: https://wenku.baidu. com/view/ba48287e7e21af45b307a820.html\#

Tomlinson, J. (1996). Cultural Globalisation: Placing and Displacing the West. The European Journal of Development Research volume, 22-35.

Trahar, S. (2009). Beyond the Story Itself: Narrative Inquiry and Autoethnography in Intercultural Research in Higher Education. Bristol: University of Bristol.

Williams, S. (2018, January 25). Augmented reality app brings Bastion Pt history to life. Retrieved from Stuff: https://www.stuff.co.nz/auckland/local-news/east-bays-courier/100833086/ augmented-reality-app-brings-bastion-pt-history-to-life

Dai X, Zhou M, Ma W \& Zhou L (2016). Influence of spatial variation in land-use patterns and topography on water quality of the rivers inflowing to Fuxian Lake, a large deep lake in the plateau of southwestern China. Ecological Engineering, 417-428.

Xinhua. (2020, March 10). Dianchi Water Quality IV. Retrieved from Xinhua News: http://www. xinhuanet.com/local/2020-03/10/c_1125690926.htm

Yang, Q. (2018). Chengjiang Plants Research. Kunming: Southwest Foresty University.

Yao, A. (2005). Scratching beneath iconographic and textual clues: A reconsideration of the social hierarchy in the Dian culture of Southwestern China. Journal of Anthropological Archaeology, 378405.

Yilmaz, R. M. (2016). Educational magic toys developed with augmented reality technology for early childhood education. Computers in Human Behavior, 240-248.

Yishiweijian. (2018, December 12). Bronzes on Yunnan Museum. Retrieved from Baijiahao: https:// baijiahao.baidu.com/s?id=1619617458951928419\&wfr=spider\&for=pc 
Cui, Y. D (2008). Macrozoobenthic community of Fuxian Lake, the deepest lake of southwest China. Ecology and Management of Inland Waters, 116-125.

Hou ,X, David J., Richard J. Ma X \& Purnell M(2017). The Cambrian Fossils of Chengjiang, China The Flowering of Early Animal Life. Oxford: John Wiley \& Sons Ltd.

Zai, J. (2015). Taoism and Science: Cosmology, Evolution, Morality, Health and more. Ultravisum.

Zakiah Noh, M. S. (2009). A Review on Augmented Reality for Virtual Heritage System. Learning by Playing. Game-based Education System Design and Development , 50-61.

Zhang, X. Z. (2010). How Does Globalisation Affect Regional Inequality within A Developing Country? Evidence from China. The Journal of Development Studies, 47-67.

Yunnanwang. (2015, November 17). protection regulation of Fuxian Lake. Retrieved from Ren Min Net: https://baike.baidu.com/reference/10453275/545cSVqOz818wPVg_8enbTQEpNSfSH EOOTQjGOUUnF-WRAqvMEUI58ZWGHplcEv66sq_c015YvuVYrualNxh/ijX_SZZnyrxyVfDxnay5i6xnprPTjGeZvFk5MGAvtOxkHo

Vannier, J., Steiner, M., Renvoisé, E., Hu Shi $\nabla x u e$ \& Casanova, JDP. 2007. Early Cambrian origin of modern food webs: evidence from predator arrow worms. Proceedings of the Royal Society

Walliss J, Rahmann H. (2016). Landscape architecture and digital technologies: Re-conceptualising design and making (Vol. 265). Abingdon, Oxon ; New York : Routledge.

de Souza e Silva, A. (2006). From Cyber to Hybrid: Mobile Technologies as Interfaces of Hybrid Spaces. Space and Culture, 9(3), 261-278.

Stuart E. (2012). Augmenting Phenomenology: Using Augmented Reality to Aid Archaeological Phenomenology in the Landscape. Pringer Science \& Business Media B.V., 582-600. https://doi. org/10.1007/s10816-012-9142-7

Xu G, Chen, Yanhui, \& Xu L. (2018). Understanding Chinese Culture Philosophy, Religion, Science and Technology (Vol. 265). Singapore : Imprint: Palgrave Macmillan.

Qiao, Y. (2015). Taoist buildings: The architecture of China's indigenous religion. New York : CN Times Books.

Zai, J. (2015). Taoism and Science: Cosmology, Evolution, Morality, Health and more. Ultravisum.

Chenoweth R \& Gobster P H (2019). The Nature and Ecology of Aesthetic Experiences in the Landscape. Landscape Journal, 38. 
Potteiger, M., \& Purinton, J. (1998). Landscape narratives: Design practices for telling stories. J. Wiley.

Lou Q. (2011). Chinese gardens. Cambridge, UK ; New York : Cambridge University Press.

Koolhaas R, Hans K, Peter R, Authur O \& Florian H. (2013). The city in the city : Berlin : a green archipelago. Zürich: Lars Müller Publishers.

Jones P (2005). Introducing social theory. New Delhi: Atlantic.

J. M. Zheng, K. W. Chan \& I. Gibson, "Virtual reality," in IEEE Potentials, vol. 17, no. 2, pp. 20-23, April-May 1998, doi: 10.1109/45.666641.

Ctrip. (n.d.). Retrieved from Wetland Park in Lake Fuxian Ctrip: https://you.ctrip.com/sight/

chengjiang2171/1416854.html

People's Government of Yunnan Province (2016, April 15). Introduction of Yuxi.

Retrieved from People's Government of Yunnan Province Website: https://

baike.baidu.com/reference/13139/2e52uUvOc_DrWTauMMQ7whKHKTjZPKdfe

HIg5r7jLOAUwwjOg_1ssBAj2YFCrhikfTvxGwb4jBiEpEb4OZ31oCliljnKtGnsyee7gN̄Wv0ihgV_

alQ8WCWMWOMQD

Vlahakis V, Karigiannis I, Tsotros M, Gounaris M \& Almeida L (2001). Archeoguide: first results of an augmented reality, mobile computing system in cultural heritage sites. proceeding of the 2001 conference on Virtual Reality, archelogy, and cultural heritage, 131-140.

Riva G, Bonas R M, Botella C, Mantovoni F \& Gaggioli A. (2016). Transforming Experience: The Potential of Augmented Reality and Virtual Reality for Enhancing Personal and Clinical Change. Psychiatry(https://doi.org/10.3389/fpsyt.2016.00164).

\section{FIGURES}

figure 47 - Bencai,Z (2007). Retrived from : http://www.99zihua.com/product-16887.html 